\title{
Rethinking the food system: \\ An Operations Research Approach
}

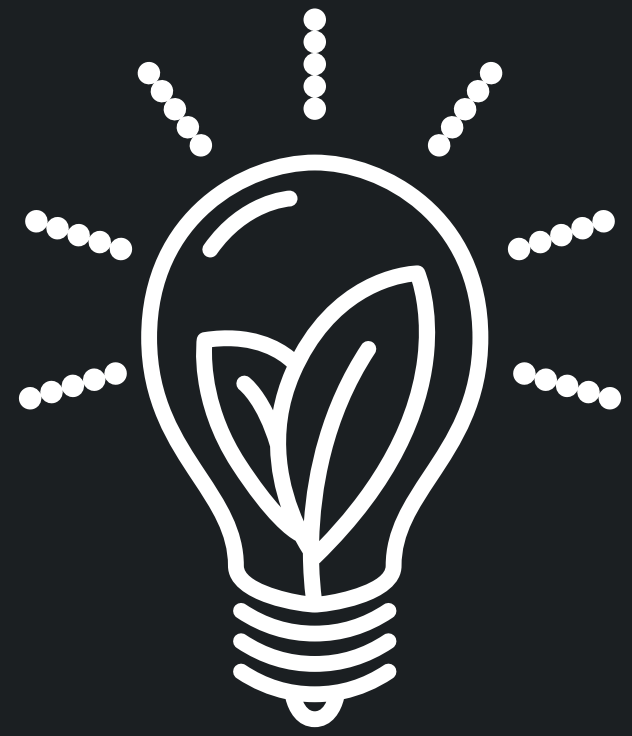





\section{Propositions}

1. The current UN definition of food security (World Food Summit, 1996) is unsustainable and based on privilege.

(this thesis)

2. There are no sustainable solutions.

(this thesis)

3. Decision support both increases and decreases the human ability to make decisions.

4. Academia limits itself through its frameworks.

5. Creating problems leads to better solutions.

6. Happiness is a multi-criteria stochastic problem.

Propositions belonging to the thesis, entitled

Rethinking the food system: An Operations Research approach

Sonja U K Rohmer

Wageningen, 20 September 2019 



\section{Rethinking the food system: \\ An Operations Research approach}




\section{Thesis committee}

\section{Promotors}

Prof. Dr J.M. Bloemhof

Professor of Operations Research and Logistics

Wageningen University \& Research

Prof. Dr P. van 't Veer

Professor in Nutrition, Public Health and Sustainability

Wageningen University \& Research

\section{Co-promotor}

Dr G.D.H. Claassen

Associate professor, Operations Research and Logistics Group

Wageningen University \& Research

\section{Other members}

Prof. Dr J.M. Geleijnse, Wageningen University \& Research

Prof. Dr H. Fleuren, Tilburg University

Prof. Dr K. Sörensen, University of Antwerp, Belgium

Prof. Dr D. Vigo, University of Bologna, Italy

This research was conducted under the auspices of the Wageningen School of Social Sciences (WASS) 


\title{
Rethinking the food system: An Operations Research approach
}

\author{
Sonja U K Rohmer
}

Thesis

submitted in fulfilment of the requirements for the degree of doctor

at Wageningen University

by the authority of the Rector Magnificus

Prof. Dr A.P.J. Mol,

in the presence of the

Thesis Committee appointed by the Academic Board

to be defended in public

on Friday 20 September 2019

at 4 p.m. in the Aula. 
Sonja U K Rohmer

Rethinking the food system: An Operations Research approach 188 pages.

PhD thesis, Wageningen University, Wageningen, The Netherlands (2019) With references, with summary in English

ISBN 978-94-6395-102-9

https://doi.org/10.18174/498977 


\section{Contents}

Page

Contents $\quad$ v

1 Hors d'Oeuvre - A General Introduction 1

1.1 Amuse-Bouche - Recipe for Disaster . . . . . . . . . . . . . . . . 2

1.2 A lot on our Plate . . . . . . . . . . . . . . . . . . . . . . . . . . . . . . . . . . . . . . . . . .

1.3 Apéritif - Serving up Solutions . . . . . . . . . . . . 4

2 Network Perspective - Sustainable Supply Chain Design in the Food $\begin{array}{ll}\text { System with Dietary Considerations } & 7\end{array}$

2.1 Introduction . . . . . . . . . . . . . . . . . . . . 9

2.2 Literature Background . . . . . . . . . . . . . . . . . . . . . . . . . . . . . . . . . . . . .

2.3 Formal Problem Description . . . . . . . . . . . . . . . . . . . . . 15

2.4 Mathematical Formulation . . . . . . . . . . . . . . . . . . . 19

2.5 Illustrative Case Description and Data Input . . . . . . . . . . . . . . . 22

2.6 Numerical Analysis . . . . . . . . . . . . . . . . . . . . . 25

2.7 Discussion and Conclusion . . . . . . . . . . . . . . . . . 35

3 Network Perspective - A Nutritional Comparison and Production Per$\begin{array}{ll}\text { spective } & 39\end{array}$

3.1 Introduction . . . . . . . . . . . . . . . . . . . . 41

3.2 Materials and Methods . . . . . . . . . . . . . . . . . . . . . . 42

3.3 Results and Discussion . . . . . . . . . . . . . . . . . . . . . . . . . . . . . . . .

3.4 Conclusion and Future Research . . . . . . . . . . . . . . . . . . 54

4 Operational Aspects - A Two-Echelon Inventory-Routing Problem for Perishable Products $\quad 57$

4.1 Introduction . . . . . . . . . . . . . . . . . . . . . . . 59

4.2 Formal Problem Description . . . . . . . . . . . . . . . . 63

4.3 Mathematical Formulation . . . . . . . . . . . . . . . . 65

4.4 Heuristic . . . . . . . . . . . . . . . . . . 67 
4.5 Computational Results . . . . . . . . . . . . . . . . . . . . . 74

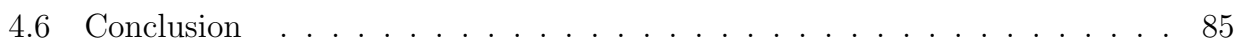

5 Operational Aspects - Last Mile Distribution with Delivery Options for $\begin{array}{lr}\text { Fresh Products } & 87\end{array}$

5.1 Introduction . . . . . . . . . . . . . . . . . . . . . . . . . . . . 89

5.2 Formal Problem Description . . . . . . . . . . . . . . . . 91

5.3 Mathematical Formulation . . . . . . . . . . . . . . . . . . . . . . . . 92

5.4 Heuristic . . . . . . . . . . . . . . . . . . . . . . . . 95

5.5 Computational Results . . . . . . . . . . . . . . . . . . . . 97

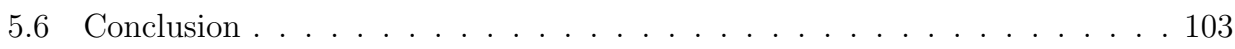

6 Product Concepts - A Model for Composing Meat Replacers 105

6.1 Introduction and Background . . . . . . . . . . . . . . . . 107

6.2 Methods . . . . . . . . . . . . . . . . . . . . . 109

6.3 Results . . . . . . . . . . . . . . . . . . . . . . . . . . . . . . . . . . . . . . . . . . . . . . . . . . .

6.4 Discussion . . . . . . . . . . . . . . . . . . . . . . 126

6.5 Conclusion . . . . . . . . . . . . . . . . . . . . . . . . . . 129

6.A Appendix - Required Contents of Nutrients . . . . . . . . . . . . 130

6.B Appendix - Life Cycle Assessment and Environmental Impact Indicators . 131

6.C Appendix - Nutritional and Environmental Data . . . . . . . . . . . . . . 134

6.D Appendix - Payoff Matrices . . . . . . . . . . . . . . . . . . 137

6.E Appendix - Sensitivity Analysis . . . . . . . . . . . . . . . 138

6.F Appendix - Compositions of the Meat Replacers . . . . . . . . . . . . . . . 141

6.G Appendix - Environmental Impact of Soy . . . . . . . . . . . . . . . . . 142

7 Digesting the Pieces - A General Discussion and Conclusion $\quad 145$

7.1 Lasting Flavours - A Bite-Sized Conclusion . . . . . . . . . . . . . . . 146

7.2 Catabolism - Breaking down the Compounds . . . . . . . . . . . . 146

7.3 Anabolic Interactions - Synthesising Valuable Contributions . . . . . . . . 154

7.4 Digestif - Tomorrow's Headache . . . . . . . . . . . . . . 156

$\begin{array}{ll}\text { References } & 159\end{array}$

$\begin{array}{ll}\text { Summary } & 179\end{array}$

$\begin{array}{ll}\text { Publications } & 183\end{array}$

$\begin{array}{ll}\text { Acknowledgements } & 185\end{array}$ 


\section{Chapter 1}

\section{Hors d'Oeuvre}

A general introduction 


\subsection{Amuse-Bouche - Recipe for Disaster}

Access to food is a driver of progress and development as well as a source of crises and conflicts in this world (FAO, 2017). As a result, food supply has always been a major challenge for mankind. Influenced by social, economic, environmental and technological developments, the way we organise this supply has evolved over time from short local chains into a complex global construct. Today's global food system is an intrinsic web of diverse supply chain activities related to the production, processing and distribution of a large variety of products (FAO, 2018). Feeding the majority of this planet's population, it provides jobs and livelihoods across countries and contributes a major share to the economy. However, increased productivity and economic gain have come at a price, paid by the natural environment. The current population dynamics, in the form of a combined population and income growth, intensify the need for higher outputs and increase the pressures already faced by the environment (FAO, 2017; Ingram, 2011). The changing environmental conditions, due to climate change, resource depletion and biodiversity loss, on the other hand proceed to hamper the system's productivity and constrain the future supply of food, threatening the socioeconomic status of future generations (Ingram, 2011).

Yet, despite these imminent strains on the supply of food, losses and waste continue to claim a considerable share of the system's output (FAO, 2011; Parfitt et al., 2010). In particular the final stages of the system often lack the necessary efficiency and account for a major portion of this waste in the form of highly processed and value added products (FAO, 2011; Parfitt et al., 2010). The perishable nature of many food products in turn complicates efficient planning, due to special handling and storage requirements. To make matters worse, current socioeconomic trends, associated with urbanisation and changing consumer lifestyles, impact the existing infrastructure and alter consumer needs and preferences (FAO, 2017). Consequently, efficient planning and customer-specific solutions gain in importance, as cities become more populated and urban transport networks suffer from congestion and pollution, while an increasing amount of consumers opts for the convenience of home delivery.

The increasing demand for comfort and convenience, however, often also manifests itself in unhealthy consumer choices and thus fosters the prevalence of diet-related noncommunicable diseases and malnutrition in the form of obesity and nutrient deficiencies. At the same time, growing societal awareness with regard to health and sustainability issues increases the focus on our dietary choices and encourages the development of new and innovative product concepts that facilitate a more aware consumption. 


\subsection{A lot on our Plate}

The interplay of social, environmental and economic pressures demands a rethinking of the current food system that takes the new circumstances into account and ensures a sustainable development, minimising the negative implications of the system. Reconsidering the setup and evaluating possible alternative scenarios is, however, a multifaceted undertaking that concerns decision making at different levels and of different scope.

Starting from a network perspective, the task of designing a more sustainable food system is confronted with plentiful choices and multiple conflicting objectives. Consumption and supply chain decisions are closely intertwined and need to take relations between products and a multitude of interconnected supply chain activities into account. Moreover, the available infrastructure differs between countries and the choice of production/processing locations determines transportation distances. Optimising the flow of products through the network thus depends on the interaction of a diverse range of aspects and their combined contribution to the overall objective. Sustainability and in particular the environmental impact of the system can, however, be characterised by a variety of factors, constituting different objectives, that pose distinct requirements to the strategic setup of the network.

Shifting perspective towards the final distribution of products, in order to address the urban challenges of the future, operational aspects gain in importance. Operational decisions have to be made on a recurring basis and require a high level of functionality. Product characteristics, such as perishability and special handling or storage requirements, play a crucial role in this context, posing additional constraints to the problem of finding good solutions. Catering to customers' individual time schedules and increasing expectations can be challenging for smooth operations but is often crucial for businesses in order to gain their competitive advantage. Innovative delivery concepts, giving more flexibility and choice to consumers, in terms of when and where products should be delivered, add more variables to the problem but may help in finding better overall solutions.

Zooming more closely into consumers' plates, specific ingredients make up a big part of common meals and account for a large portion of the environmental impact associated with our current diet. The development of innovative product concepts that are able to replace these ingredients within traditional meals can provide consumers with easy alternatives and hence help to reduce the environmental impact. Designing these alternatives, means making choices about a large number of ingredients that are able to provide the nutrient equivalence as well as a similar structure, let alone taste, as the original product. Moreover, depending on the environmental objectives used for the decision-making, different alternatives are possible and trade-offs may occur. 


\subsection{Apéritif - Serving up Solutions}

Operations Research approaches can facilitate complex problem-solving and decision making and, thus, help in finding good solutions to the problems society is facing today. Focusing on rethinking the food system, this research aims to address the current challenges with the help of mathematical optimisation and solution techniques, investigating possible alternatives for a more sustainable future. By approaching the topic from different angles, this study covers a broad spectrum of problems at different levels of the system, varying in size and scope.

The research framework of this thesis, (shown in Figure 1.1) gives an overview of these levels and the general scope of the conducted research. In the figure, the considered food system is represented by the dashed box, comprising the decisions in the system, the decision makers and the specific system characteristics. Given the emphasis on decision making and complex problem solving, the decisions in the food system constitute the focal point of this thesis. In order to address the different pressures on the system (outlined in the red box), the thesis considers supply chain and consumption decisions within a common framework and investigates decision problems at the strategic, operational and individual level. By addressing these different decision levels, the research covers different views of the system and attempts to include the perspective of different decision makers (light blue box), thus, sharing the responsibility of rethinking the system between the different actors in the system. The system characteristics on the other side (dark blue box) restrict the decisions in the form of constraints, that have to be taken into account when reconsidering the system.

Following from this, the overall aim of this research is to propose adequate optimisation models and solution techniques that can facilitate the rethinking of supply chain and consumption decisions at the strategic, operational and individual level of the food system. 


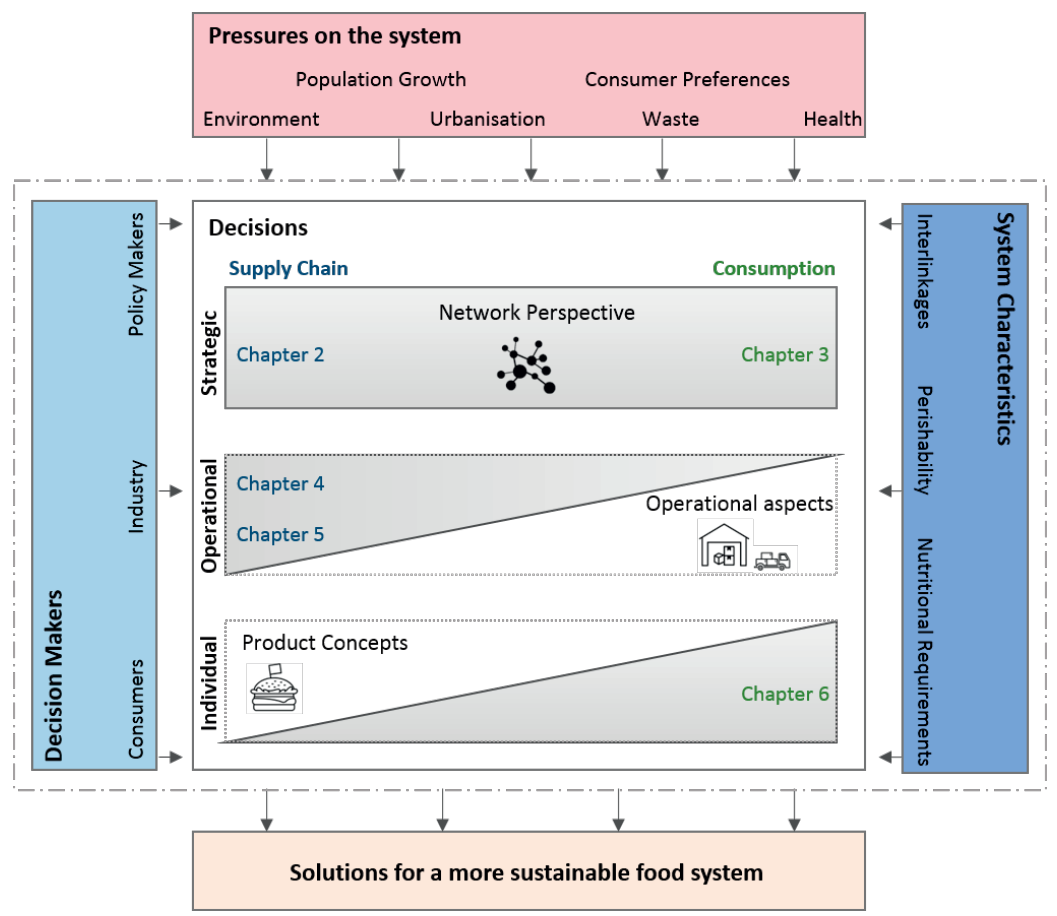

Figure 1.1: Conceptual research framework for rethinking the food system

The following presents a more detailed outline of the chapters included in this thesis, with Figure 1.2 providing a graphical representation of the different views on the system and the order in which they are discussed in this thesis.

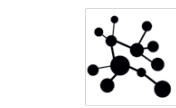

Network perspective

Chapter $2 \& 3$

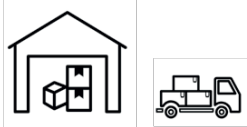

Operational aspects

Chapter 4 \& 5

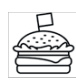

Product Concepts

Chapter 6

Figure 1.2: Steps towards a sustainable food system: An outline of this thesis

Focusing on the network perspective, Chapter 2 and 3 investigate the impact of shifting towards a more plant-based dietary consumption on the basis of a number of alternative scenarios. Optimising the flow of products through the network, the chapters highlight the relationship between consumption and supply chain decisions and their impact on the environment. The analysis further includes insights into different environmental burdens, 
their allocation in the system and potential trade-offs between the conflicting objectives. In this context, Chapter 2 focuses more on production aspects associated with the different supply chain activities in the network, while also providing a more in depth multi-objective analysis. Chapter 3 in contrast concentrates more on the consumption side of the network and the implications for the overall nutritional intake.

Looking into operational aspects within the system, Chapter 4 and 5 present innovative concepts for the last mile distribution of perishable products. Assuming a two-echelon distribution structure, Chapter $\mathbf{4}$ focuses on the integrated optimisation of inventory and routing decisions in the last mile context, under consideration of customer specific delivery patterns. Due to the complexity of the problem, the emphasis of the study lies primarily on the development of adequate solution approaches for the problem. Proposing a system with alternative delivery locations, Chapter $\mathbf{5}$ provides more managerial insights, by investigating the impact of routing and location decisions on distribution costs.

Dealing with the development of innovative product concepts, Chapter 6 covers the design of environmental friendly alternatives to meat. Composing meat replacers with an equivalent nutritional value to meat, the study gives particular attention to protein quality and amino-acid composition. By optimising a number of selected impact indicators, the chapter further highlights existing trade-offs between different environmental burdens.

Building on the insights obtained from solving these decision problems, Chapter 7 presents a general discussion and conclusion of the most relevant findings and identifies possible directions for future research in the field. 


\section{Chapter 2}

\section{Network Perspective}

Sustainable supply chain design in the food system with dietary considerations: A multi-objective analysis

This chapter is published as:

Rohmer, S. U. K., Gerdessen, J. C., \& Claassen, G. D. H. (2019). Sustainable supply chain design in the food system with dietary considerations: A multi-objective analysis. European Journal of Operational Research, 273(3), 1149-1164. 


\section{Abstract}

Food is a vital component of everyday life, however current consumption and production patterns pose a threat to the environment and the food security of future generations. Thus, with environmental burdens becoming more apparent and rising societal awareness, it is time to reconsider dietary choices and the food system behind it. This chapter presents a novel application of a network design problem, addressing sustainability issues in the context of the global food system. Taking into account several echelons and interlinkages between different food supply chains, the chapter broadens the scope of the considered network and incorporates sourcing, processing and transportation decisions within a common framework. While minimising different environmental and economic objectives, the model aims to maintain a sufficient dietary intake level. Consumption decisions are incorporated in the model through different types of consumer demands. The problem is formulated based on linear programming and further analysis is carried out by applying the $\epsilon$-constraint method and compromise programming. Investigating alternative production and consumption scenarios as well as trade-offs between the conflicting objectives, the study is illustrated based on a nutritional case study and underpinned by real-life LCA data. The findings of this research are manifold, highlighting the importance of considering consumption and production decisions in an integrated and global setting. Moreover, the choice of sustainability indicator plays a crucial role given the often conflicting nature of different sustainability aspects. 


\section{$2.1 \quad$ Introduction}

Food does not only contribute significantly to our health and well-being but also plays a crucial role in global and local economic markets. However, with food systems being highly resource dependent, our diet also has implications for the environment we live in, both directly through the amount and combination of plant and animal products we consume and indirectly through the production of these products (Alder et al., 2012). On a global scale, food systems account for about $24 \%$ of the greenhouse gas emissions, $33 \%$ of the soil degradation as well as $60 \%$ of the terrestrial biodiversity loss (UNEP, 2016), while on a European level the food sector, and agriculture in particular, continues to be one of the most water and energy demanding sectors (Maguire et al., 2017). Meat and dairy products are among the products with the highest contribution to these environmental burdens (Notarnicola et al., 2012; Steinfeld et al., 2006). A growing population combined with current unsustainable and wasteful food consumption and production patterns, marked by overconsumption and excessive consumption of meat and dairy products, aggravate these environmental threats and put further pressure on our environment in the form of global warming, resource depletion and the extinction of species (UNEP, 2016). Sustainable development, addressing economic, environmental and social issues, thus receives growing attention in the context of the food system.

The food system, however, with its transnational nature, is a highly complex and dynamic network involving multiple agents, a wide product variety and a large number of processes, ranging from production or manufacturing processes to logistic and retail activities (Trienekens et al., 2012). Furthermore, globalisation and differences in the affected social and ecological systems, such as climatic and geographical conditions or the development status of a country, affect the transparency and complicate sustainable decision making in the food system. A tomato grown locally in a greenhouse in the Netherlands, for example, will have a different environmental footprint than a tomato grown in Italy or Spain, as the activities and processes involved, such as transportation or energy input during production, will have different contributions to the overall environmental impact. From a social perspective, health aspects and nutrition play the most prominent role in the food system with the UN defining food security and improved nutrition as one of its sustainable development goals (UN, 2015). There are many other aspects that could be considered in the context of the food system, such as equality between and within countries, working conditions or fair trade, however, these social impacts are often hard to quantify, measure and aggregate on a global scale. This research will thus focus on the nutrition and health aspect.

Economically, costs remain the key factor in the decision making process as profit margins for food products are often low, competition is high and the affordability of food in general is a key issue in today's society. It is therefore necessary to consider cost, nutritional and 
environmental aspects together in order to make sustainable decisions about the design of our future food system. The conflicting nature of these objectives, however, complicates this process.

Using traditional Operations Research techniques in combination with LCA data, this research presents a novel application in the context of a sustainable food system. The chapter addresses a multi-objective network design problem for the food system under consideration of product and nutrient demands, broadening the scope of the considered network by taking into account interlinkages between different food supply chains. The proposed model incorporates sourcing, processing and transportation decisions, minimising both environmental (e.g. land use, climate change, fossil fuel depletion, etc.) as well as cost aspects while respecting the nutritional requirements of the society. Building on the work of other research, we aim to investigate the impact of a shift from meat-based to plant-based dietary consumption on the supply chain configuration. We illustrate the food system based on a nutritional case study and underpin it with real-life LCA data. In our analysis we investigate trade-offs between the conflicting objectives and highlight possible shifts from one environmental burden to another. Furthermore, we provide an overview over the allocation of these burdens in the network and the contribution of the different phases within the network's configuration.

The chapter is organised as follows. In section 2.2 a short literature background will be given. Section 2.3 will give a formal description of the problem. In section 2.4 the multiobjective linear programming model will be introduced. Section 2.5 gives an overview of the experimental setup and the data input, before Section 2.6 presents the numerical analysis and findings from the model. Section 2.7 contains concluding remarks and some promising directions for future research.

\subsection{Literature Background}

\subsubsection{Sustainable supply chains}

Over recent years, literature on supply chains and sustainability has received increasing attention within the scientific community, indicating an emerging trend within the field of green and sustainable supply chain management, design and planning. In this context, a number of review papers have been issued providing an overview over the current stateof-the-art. Table 2.1 provides a general overview of these review papers on sustainable supply chain management presenting the main insights for each study and the relevant findings in the context of this research. 
Table 2.1: Literature reviews on sustainable supply chain management (SSCM)

\begin{tabular}{|c|c|c|}
\hline Author(s) & Year & Insights and Findings \\
\hline Srivastava & 2007 & $\begin{array}{l}\text { Review and classification of green supply chain management literature, providing an evolutionary timeline } \\
\text { with focus on the environmental dimension. }\end{array}$ \\
\hline Seuring and Müller & 2008 & $\begin{array}{l}\text { Review and conceptualisation of a total of } 191 \text { papers in the area of SSCM, highlighting the relevance of } \\
\text { government influences and other stakeholder groups. The review shows a clear under representation of the } \\
\text { social dimension in the literature. }\end{array}$ \\
\hline Mollenkopf et al. & 2010 & $\begin{array}{l}\text { Review of the literature with emphasis on the relationships between green, lean and global supply chains, } \\
\text { showing a need for more multi-functional approaches and strategic integration in a global context due to } \\
\text { existing trade-offs between the different functional levels (such as for example purchasing and logistics), } \\
\text { also calling for the development of more holistic systems approaches. }\end{array}$ \\
\hline
\end{tabular}

Carter and Liane Easton 2011 Systematic review of the SSCM literature, using risk aspects and organisational structures to conceptualise sustainable operations

Ashby et al.

2012 Systematic review of the current literature on supply chain management with social and environmental sustainability considerations. The work identifies a tendency towards theory and qualitative approaches, with most of the current literature focussing on "just the 'greening' of supply chain processes". The research denotes a clear need for more holistic approaches, that take supply chain relations into account, while the authors specify the potential benefits of LCA and closed loop approaches in this context.

Dekker et al.

Hassini et al.

Tang and Zhou

Seuring

Brandenburg et al.

Brandenburg and Rebs

Eskandarpour et al.

Jaehn
2012 Review of Operations Research contributions to the field of green logistics, thus addressing environmental aspects affecting design, planning and control decisions along the supply chain, including transportation, inventory and facility considerations. The study mentions the importance of metrics and multi-criteria decision making approaches in this context.

2012 Literature review on SSC primarily elaborating on adequate metrics for sustainable operations, proposing two frameworks for the management and relevant performance measures in supply chains. The authors also note the need for more industry specific research due to different supply chain requirements.

2012 Presentation of a profit-planet-people framework to understand the interrelations of activities impacting sustainability aspects, in combination with a categorisation of recent Operations Research literature with focus on quantitative models. The research shows that most literature fails to address the people dimension and lacks multi-location systems that take interactions in the supply chain into account.

2013 Review of modelling approaches for SSCM, revealing that LCA is the most often applied modelling technique while most studies assess trade-offs between different sustainability issues with the social dimension not being enough accounted for.

2014 Review and categorisation of a total of 134 papers with focus on formal models in the area of SSCM, showing that multi-criteria decision making and LCA are the most commonly used tools for modelling. Based on the review, the research concludes that there is a lack of social aspects and inter-organisational perspective in the model-based literature.

2015 Review assessing and clustering 185 journal publications on quantitative modelling in the area of SSCM. The work identifies a lack of social aspects and denotes a need for more comprehensive models to describe the impact of entire sectors or industries.

2015 Review of 87 papers in the area of SSC network design, focusing in particular on LCA based approaches. Findings show that the sustainability indicators are mostly still limited to greenhouse gas emissions, thus there is a need for the inclusion of broader life-cycle perspectives and social aspects.

2016 Conceptualisation of the field of sustainable operations addressing the interactions between the three sustainability dimensions. The paper is structured according to the fields within sustainable operations focusing on the use of operations research models and highlighting the main objectives for each dimension within the specific field. 
The insights from the literature reviews show that most research fails to include the social component, focusing predominantly on environmental and economic aspects, which might be partly due to difficulties in the measurement and determination of the relevant social factors (Jaehn, 2016). Furthermore, there is a need for more holistic models, extending the system both in terms of the supply chain echelons considered as well as with respect to inter-organisational interactions and relations or global considerations. From a modelling perspective, multi-criteria decision making techniques and LCA approaches are the most widely applied methods and show further potential to support decision making in the field of SSCM. More industry specific and empirical research is needed in order to account for specific supply chain requirements. In this chapter we will try to address some of these issues by considering a more holistic model within a global setting that extends the system, taking more echelons and interrelations into account. Focusing on the food industry, we make use of real life LCA data and investigate the application of multi-criteria decision making approaches to the problem at hand. In the following sections we will thus give a more detailed overview of these requirements in the context of agricultural and food supply chains and introduce the relevant literature and insights, followed by a brief overview of the use of multi-criteria decision making (MCDM) approaches in the field.

\section{Sustainablity in agricultural and food supply chains}

Focusing on applications in the area of production and distribution planning for agrifood supply chains, Ahumada and Villalobos (2009) present a review and categorisation of the relevant literature in the field. The study concludes that most approaches focus on the operational and tactical side of decision making within the supply chain framework rather than on strategic decisions and structural supply chain design issues. Presenting a critical taxonomy and hierarchical decision making framework for agrifood supply chains, Tsolakis et al. (2014) show that most of the existing research on agrifood supply chains is based on case studies focusing on specific parts of the chain rather than providing an integrated framework. Similarly, Higgins et al. (2010), focussing on Operations Research approaches for agriculture supply chains, highlight the increasing need to consider these complex systems/networks as a whole rather than solely optimising over parts of it. The work considers in particular the use of systems science methods, such as agent based modelling, dynamic systems and network theory to deal with this issue. Integrating sustainable supply chain management and dynamic capabilities within the same conceptual framework, Beske et al. (2014) conduct a review of sustainable supply chain management in the food context. Zhu et al. (2018) conduct a review of model-oriented applications of OR techniques in the field of sustainable food supply chains. Reviewing 83 papers, the research identifies the main food specific issues within the three sustainability dimensions and outlines a number of different future research directions, including the need to 
approach sustainable food supply chain design from a more global perspective. In this context of food supply chains, Dani (2015) provides a comprehensive overview over the scope of the chain decisions as well as current issues and challenges. The three main food-specific challenges for supply chain optimisation are identified as product quality, safety and sustainability (Akkerman et al., 2010; Van Der Vorst et al., 2009). Focusing on sustainability aspects, Iakovou et al. (2016) present a holistic framework for the design and operations of agrifood supply chains from an interdisciplinary perspective. The work highlights the industry specific needs and requirements with respect to policies, technologies, practices and solutions. Soysal et al. (2012) provide a literature review specifically focusing on quantitative modelling in the field of sustainable food logistics. Their findings show, that despite a growing interest in the area of food logistics, models incorporating food supply chain dynamics as well as sustainability aspects are still relatively scarce. The general trend in the scientific literature on food supply chains goes towards integrated and collaborative approaches while sustainability of the chain as a whole also receives more and more attention, as integrated approaches and collaboration between agents can yield greater benefits in terms of optimisation and raise standards (Higgins et al., 2010; Smith, 2007; Van Der Vorst et al., 2009). However, more holistic approaches focusing on the strategic decision making level are still lacking.

\section{Multi-criteria decision making approaches in sustainable supply chain design}

As literature incorporates a greater variety of aspects into the decision making processes related to supply chain management, multi-criteria decision making (MCDM) methods, such as multi-objective optimisation, are becoming increasingly popular in the area of sustainable supply chain management. Banasik et al. (2018) provide a conceptual framework and review of MCDM approaches in the field of green supply chain design and, given the new and emerging nature of the field, identify a need for more research in the area. Hayashi (2000) reviews multicriteria applications in the context of agricultural research management with the aim to evaluate and classify the criteria used. The study includes both multi-attribute and multi-objective methods. Linnemann et al. (2015) show the potential of MCDM for evaluating alternatives and increasing transparency in the context of food supply chain design by applying Multi Attribute Value Theory (MAVT) and Analytic Hierarchy Process (AHP) to the case of Novel Protein Foods. Mallidis et al. (2012) propose a multi-objective model for supply chain design minimising cost as well as emissions and apply it to a supply chain network in South-Eastern Europe. Nagurney and Nagurney (2010) present a multi-criteria modelling framework for supply chain network optimisation with capacity considerations, optimising both cost as well as emissions of various chain activities. Oglethorpe (2010), presents the use of goal programming to address different economic, environmental and social goals in the context of alternative 
food supply chain strategies, applying the concept to local, regional and national decision making levels. Validi et al. (2014) consider a two-layer food supply chain distribution system in the context of multiple objectives, optimising both costs and emissions of the distribution routes. Soysal et al. (2014) present a multi-objective model for perishable products with application to the beef network. The model minimises cost and emissions under consideration of load factors, road structures and fuel types. Minimising cost, emissions and delivery time, Bortolini et al. (2016) propose a multi-objective model for multi-produce, multi-level distribution network planning for perishable products. The research is applied to the case of a consortium distributing fresh products to European retailers. Allaoui et al. (2018) develop a two stage hybrid multi-objective approach based on the AHP method and a multi-objective optimisation model for the design of sustainable agri-food supply chains. The research includes social aspects in the objectives by considering the number of jobs created in addition to the water footprint, emissions and economic costs. In conclusion it can be said, that MCDM approaches are a prevalent and useful tool to deal with the different and often conflicting objectives and criteria in the field of sustainable supply chain design. However, the objectives addressed in the literature are mostly still limited to cost and greenhouse gas emissions.

\subsubsection{Sustainable food consumption}

With regards to food consumption a wide variety of contributions to the literature was made over recent years analysing the composition of diets according to a number of different criteria such as health, cost and sustainability. In this context, MacDiarmid et al. (2012); Ribal et al. (2016); Tyszler et al. (2016) and Wilson et al. (2013) present decision/diet models based on linear programming techniques, in order to determine the optimal composition of human diets under sustainability considerations, thus taking into account costs, greenhouse gas emissions and nutritional aspects. The findings of these studies show that switching to a more plant-based diet generally has the highest potential to reduce the environmental impact and can be achieved without a loss in the nutritional value. Hallström et al. (2015) show similar findings based on their review investigating 49 dietary scenarios with respect to their environmental impact in terms of land use and greenhouse gas emissions. Life cycle analysis (LCA) is often used to evaluate food choices based on their environmental impact and provides a number of different indicators for the sustainability assessment of foods (Mogensen et al., 2009). While these indicators are dependent on the life cycle of the product and therefore closely linked with the supply chain, Verkerk et al. (2009) show, based on an example, that links also exist between supply chain design in the food industry and impacts on nutritional intake and human health. Given these links, consumption and production choices should be considered in an integrated framework (Clark, 2007). In general, research on sustainable diets fails however 
to consider the impact of the underlying production system or interrelations between products, while the environmental aspects considered are mostly limited to greenhouse gas emissions.

\subsection{Formal Problem Description}

Given the gaps identified in the literature background, this study aims to present an integrated modelling approach, addressing the global food system, consisting of production, distribution and consumption activities, in a more holistic way by taking into account the different stages and inter-linkages in the underlying supply network. Individual food chains are composed of several steps, starting with agricultural production followed by transportation and further processing of produce, before the final distribution to retailers and customers occurs. Transportation can often be done with multiple transport modes, such as for example truck, freight ship or plane. The choice of available transport mode is thus considered a variable in the model, the transport distances are mode dependent and the availability of a mode is based on country specific infrastructure. In practice, there are often many sources of agricultural goods, several processing options and multiple destinations. In addition to this, due to links and interrelations between products, the whole network can get even more complex, including several processing steps, different processing options, side streams, backward loops and by-products, linking one single product to a variety of other products. 


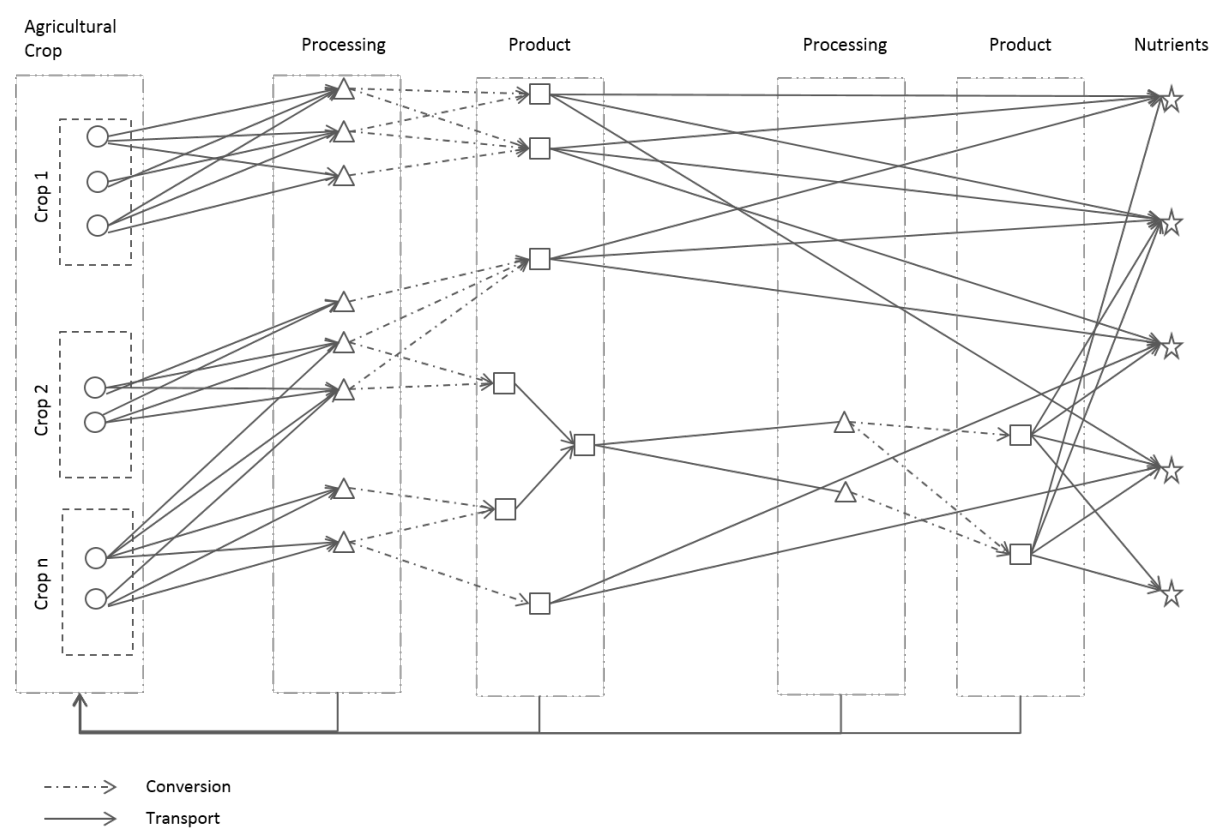

Figure 2.1: Generic food network

Figure 2.1 provides a generic representation of the food network from agricultural production up to the nutritional contribution at consumer level, including different sourcing locations, processing and product choices. The problem gets increasingly complex depending on the number of agricultural crops, products and processing steps involved in the network. The overall objective of the model is to find the optimal network design in terms of sourcing locations, transport modes and processing options as well as the optimal mix and quantity of products produced, optimising a number of sustainability indicators.

\subsubsection{Sustainability indicators}

Within the food system, decisions are guided by multiple and often conflicting objectives such as economic and environmental considerations, as these aspects are important for actors in order to stay competitive. In this context, Table 2.2 provides an overview over the selected sustainability indicators and the criteria for selection. 
Table 2.2: Selected indicators and selection criteria

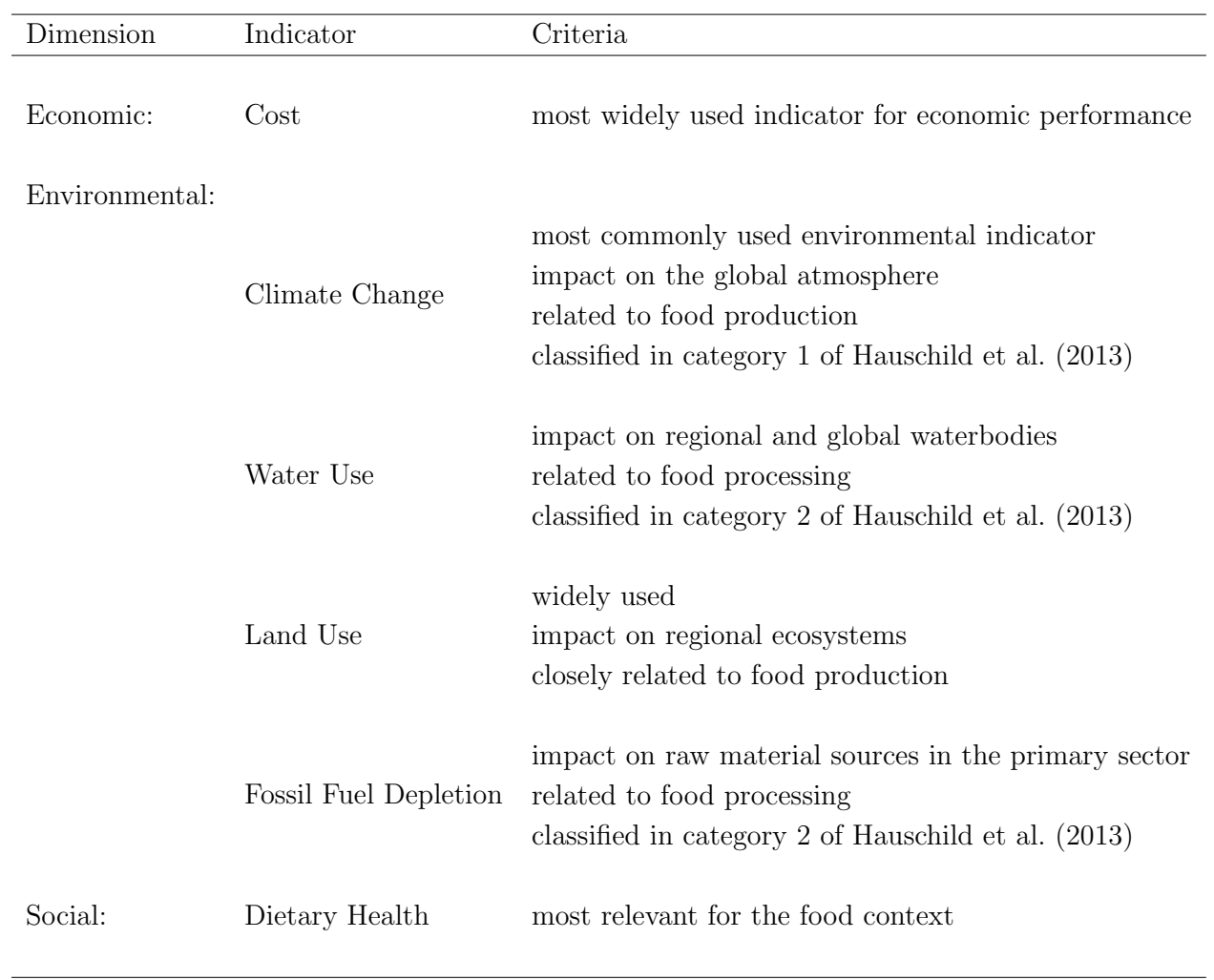

Cost is selected as the most commonly used indicator for the economic dimension. For the environmental impact indicators, the focus in this research is on climate change, land use, water use and fossil fuel depletion. The selection of environmental indicators is based on the principles and the review of LCA studies presented in Van Mierlo et al. (2017). The most important characteristics were thus the frequency of use, to facilitate comparability with other studies as well as the relevance for the food system. Furthermore, the threefold classification of Hauschild et al. (2013) considering the quality of the LCA modelling was taken into account, where category 1 represents best practice and is therefore most recommended. The environmental indicators further cover all the ecological systems categorised by Jaehn (2016). Note, that while the environmental and economic dimensions of sustainability, are included in the form of objectives in this research, the social dimension is incorporated in the form of constraints concerning the dietary/nutritional intake. Given the difficulties associated to the quantification and measurement of other social indicators, dietary health was chosen as one of the most relevant issues in the context of food. 


\subsubsection{Case study}

Given the complexity and size of today's globalised food system, the problem is reduced to a specific nutritional case study focusing on a limited number of products to choose from in order to supply only a selected number of nutrients, instead of a complete diet. While in reality the system is much more complex and involves a much larger amount of products, in this research the emphasis will be on the beef and dairy chains and a number of alternative products that are suitable to replace beef and dairy products in terms of their nutritional aspects. The reason for this choice is that, while the beef and dairy industry is an important source of certain nutrients within the human diet, such as zinc, iron, protein, vitamin B12 and calcium, it also contributes significantly to the environmental impact of the food system (Hallström et al., 2015; Notarnicola et al., 2012). A reduction in the consumption of beef and dairy products could therefore lead to substantial improvements in terms of the environmental impact of the entire system.

The beef chain in itself, however, is a fairly complex and resource intensive network. In comparison with other products, beef and dairy chains require significant input from other systems in the form of for example feed during the livestock production phase, while also producing a number of secondary products such as manure and other by-products during the processing phase further downstream, which then again link (back) into other systems. Figure 2.2 provides a simplified schematic of the beef and dairy chain from agricultural production to final consumption. 


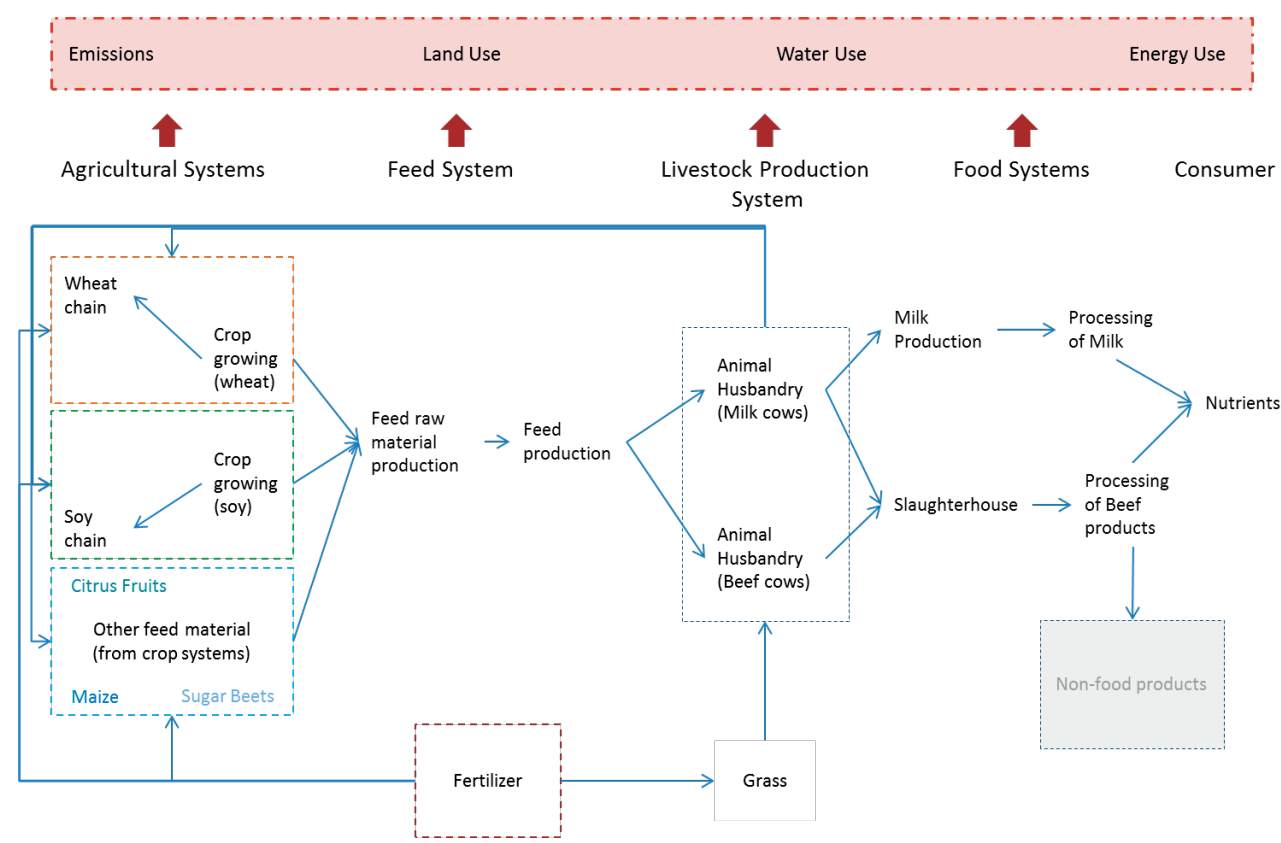

Figure 2.2: Schematic of the beef \& dairy network

The substitute products are selected from suitable plant-based alternatives with regards to the important nutrients present in beef and dairy while also taking into account current Dutch consumption patterns. The key criteria for the selection of plant-based alternatives is thus their nutrient profile, as well as their popularity and acceptance in the Dutch population (Voedingscentrum, 2016). Given that vitamin B12 is not naturally present in plant-based products, we allow for supplementation of vitamin B12.

\subsection{Mathematical Formulation}

This section provides a detailed description of the linear programming (LP) model and the notations and parameters used.

The total cost function for the network design problem is:

$$
T C=T P C+T T C
$$

The total environmental impact per indicator is given by: 
Table 2.3: Summary of notation

\begin{tabular}{|c|c|}
\hline \multicolumn{2}{|c|}{ Sets and Indices } \\
\hline A & Set of locations (countries) indexed by $i, j, l$ \\
\hline$C$ & Set of consumer locations $(C \subset A)$ \\
\hline$S$ & Set of production locations $(S \subset A)$ \\
\hline$P$ & Set of products indexed by $p, q, r$ \\
\hline$P_{0}$ & Set of processed products $\left(P_{0} \subset P\right)$ \\
\hline$m$ & Index for transport mode \\
\hline$n$ & Index for nutrient \\
\hline$f$ & Index for environmental indicator \\
\hline$k$ & Index for food category \\
\hline \multicolumn{2}{|c|}{ Parameters } \\
\hline$c p_{i, p}$ & cost of product $p$ in location $i \in S$ \\
\hline $\operatorname{conv}_{r, p}$ & conversion factor from product $r$ to product $p$ \\
\hline rat $_{p, q}$ & ratio of by-product $q$ when producing product $p$ \\
\hline $\operatorname{dist}_{i, j}$ & distance between $i$ and $j$ \\
\hline$c t_{m}$ & cost of transport for mode $m$ \\
\hline$d_{i, n}$ & nutrient demand at location $i \in C$ for nutrient $n$ \\
\hline$a_{p, n}$ & nutrient content for nutrient $n$ in product $p$ \\
\hline$e p_{i, p, f}$ & $\begin{array}{l}\text { environmental impact of product } p \text { at location } i \in S \text { for } \\
\text { sustainability indicator } f\end{array}$ \\
\hline$e t_{m, f}$ & $\begin{array}{l}\text { environmental impact of transport mode } m \text { for } \\
\text { sustainability indicator } f\end{array}$ \\
\hline$l o c_{p, i}$ & $=1$ if production of product $p$ is possible in location $i \in S$ \\
\hline $\operatorname{port}_{k, i}$ & portion size at location $i \in C$ related to food category $k$ \\
\hline$D_{k, i}$ & demand for food category $k$ at location $i \in C$ \\
\hline$\alpha$ & share of food category demand \\
\hline$b_{p, k}$ & $=1$ if product $p$ is in food category $k$ \\
\hline$r e l_{r, p}$ & $=1$ if product $r$ is a resource for product $p$ \\
\hline \multicolumn{2}{|c|}{ Decision Variables } \\
\hline$x_{i, p}$ & quantity of product $p$ produced at production location $i \in S$ \\
\hline$z_{i, p}$ & quantity of product $p$ consumed at consumer location $i \in C$ \\
\hline$y_{i, j, p, m}$ & $\begin{array}{l}\text { quantity of product } p \text { transported from } i \text { to } j \\
\text { with transport mode } m \text { in the final transport stage }\end{array}$ \\
\hline$u_{i, j, p, m}$ & $\begin{array}{l}\text { quantity of product } p \text { transported between production } \\
\text { locations } i \text { and } j \text { with transport mode } m\end{array}$ \\
\hline$v_{p, i, r}$ & $\begin{array}{l}\text { quantity of product } r \text { needed at location } i \in S \text { to produce } \\
\text { product } p\end{array}$ \\
\hline$w_{i, p}$ & amount of product $p$ wasted at location $i \in S$ \\
\hline$o_{i, n}$ & amount of nutrient $n$ consumed at location $i \in C$ \\
\hline$E P_{f}$ & $\begin{array}{l}\text { total environmental impact related to production activities } \\
\text { (including agricultural and processing) for indicator } f\end{array}$ \\
\hline$E T_{f}$ & $\begin{array}{l}\text { total environmental impact related to transportation for } \\
\text { indicator } f\end{array}$ \\
\hline$T E_{f}$ & total environmental impact for indicator $f$ \\
\hline$T T C$ & total transport cost \\
\hline$T P C$ & total production cost \\
\hline$T C$ & total cost \\
\hline
\end{tabular}




$$
T E_{f}=E T_{f}+E P_{f} \quad \forall f \in F
$$

The above functions are used as objective functions in the model and minimised separately (for cost and different environmental indicators) or in the form of constraints in the $\epsilon$ constraint method (Ehrgott, 2009).

The remaining costs are given in the following expressions:

$$
\begin{aligned}
T T C & =\sum_{i \in S} \sum_{j \in C} \sum_{p \in P} \sum_{m \in M} d i s t_{i, j} c t_{m} y_{i, j, p, m}+\sum_{i \in S} \sum_{j \in S} \sum_{p \in P} \sum_{m \in M} d i s t_{i, j} c t_{m} u_{i, j, p, m} \\
T P C & =\sum_{i \in S} \sum_{p \in P} c p_{i, p} x_{i, p}
\end{aligned}
$$

The specific environmental impact functions are given by the following:

$$
\begin{aligned}
E T_{f} & =\sum_{i \in S} \sum_{j \in C} \sum_{p \in P} \sum_{m \in M} \operatorname{dist}_{i, j} e t_{m, f} y_{i, j, p, m}+\sum_{i \in S} \sum_{j \in S} \sum_{p \in P} \sum_{m \in M} \operatorname{dist}_{i, j} e t_{m, f} u_{i, j, p, m} \quad \forall f \in F \\
E P_{f} & =\sum_{i \in S} \sum_{p \in P} e p_{i, p, f} x_{i, p} \quad \forall f \in F
\end{aligned}
$$

The demand is determined by $\alpha$, which controls the share of food category/product demand in the model, given by,

$$
\alpha D_{k, i} \leq \sum_{p \in P} b_{p, k} z_{i, p} \quad \forall k \in K, i \in C
$$

and the nutrient demand,

$$
d_{i, n} \leq \sum_{p \in P} a_{p, n} z_{i, p} \quad \forall n \in N, i \in C
$$

with the consumption of product categories constrained by portion sizes:

$$
\operatorname{port}_{k, i} \geq \sum_{p \in P} b_{p, k} z_{i, p} \quad \forall k \in K, i \in C
$$

The quantity of products consumed has to be transported to the consumer:

$$
z_{j, p} \leq \sum_{i \in S} \sum_{m \in M} y_{i, j, p, m} \quad \forall p \in P, j \in C
$$


The quantity of products transported to the consumer has to be less or equal to the quantity produced:

$$
\sum_{j \in C} \sum_{m \in M} y_{i, j, p, m} \leq x_{i, p} \forall p \in P, i \in S
$$

The blending and resource constraints ensure that all the required resources needed for production of a product are available at the production location:

$$
\begin{aligned}
& v_{p, i, r}=\operatorname{conv}_{r, p} x_{i, p} \quad \forall p \in P_{0}, r \in P, i \in S \\
& \sum_{p \in P} \operatorname{rel}_{r, p} v_{p, j, r}=\sum_{m \in M} \sum_{i \in S} u_{i, j, r, m} \forall r \in P, j \in S
\end{aligned}
$$

Produced products have to be transported to the place where they are needed as resources or consumed and otherwise are classified as waste:

$$
\sum_{m \in M} \sum_{j \in S} u_{i, j, p, m}+\sum_{m \in M} \sum_{l \in C} y_{i, l, p, m}+w_{i, p}=x_{i, p} \quad \forall p \in P, i \in S
$$

The by-product constraints link the production of a product to its by-products:

$$
x_{i, p}=\operatorname{rat}_{q, p} x_{i, q} \quad \forall p \in P, q \in P, i \in S
$$

Defining some of the variables as dynamic in order to reduce the size of the model, the final model includes over 156 thousand rows (constraints) and more than 830 thousand variables. However, given its linear and continuous nature the model can be solved quickly by any standard LP solver.

\subsection{Illustrative Case Description and Data Input}

\subsubsection{Illustrative case}

The applicability of the model is illustrated based on a real life case study related to current consumption patterns in the Netherlands. In connection to the dietary contribution of the beef and dairy system, 5 key nutrients are identified: protein, iron, zinc, calcium and vitamin B12. To allow for dietary replacement, the plant-based alternatives are selected based on their nutrient profile with respect to these 5 key nutrients. It should be mentioned that vitamin B12 is not present in plant-based products and thus needs to be supplemented in a solely plant-based dietary consumption (Broekema and Blonk, 2009). As a result, 10 food categories are selected, comprising 25 products suitable for human 
consumption. Together with the resources and feed materials needed to supply these products, this results in a network of 72 different products. Examples of such products are feed ingredients for the beef and dairy system or different grains and flour for the production of bread.

The case furthermore includes a total of 39 locations for the sourcing and production of products. These locations are product-dependent, and hence the amount and composition of feasible locations varies per product. Seven different modes of transport have been included in the model, namely air, inland waterways, rail, truck as well as three distance related sea shipping modes. The availability of a mode choice is dependent on the infrastructure and geographical position of a country, while distances between countries are location and mode dependent. Distances within a country are set to be the same for all available modes and all countries $(10 \mathrm{~km})$. On a global scale, where products are sourced from all over the world, the transportation within a country is assumed to have a minor impact in comparison to the travel distances between countries. Moreover, we assume that agricultural production locations and the facilities for further processing are likely to be located in close proximity to one another, in order to avoid product decay, save time and delivery costs. While this might be a strong assumption, it has been shown in the scientific literature that within agricultural systems the impact associated with transportation plays only a minor role (Weber and Matthews, 2008; Garnett, 2011).

\subsubsection{Data input}

The nutritional data for the selected food products were retrieved from the NEVO database (RIVM, 2013). Environmental impact values are based on country specific life cycle inventory data, containing all the inputs and outputs for each specific production and processing step. We apply the ReCiPe impact assessment method (Goedkoop et al., 2013) to translate the emissions and resource extractions into the four environmental indicators selected in section 2.3.1. The functional unit, referring to the quantification of the product to which the inputs and outputs relate, is expressed per kilogram of product. In the case of multiple products resulting from the same production step, an allocation method needs to be selected in order to divide the process inputs and outputs among the different products. The chosen allocation method in this study is economic allocation and thus based on the economic value of the products. Note, that no specific system boundaries for the scope of the LCA are applied as each step of the life cycle is included as a separate choice in the model by using life cycle inventory data, instead of using aggregated LCA impact values. All the life cycle inventory data as well as the (by-)product relations and conversion factors are extracted from the Agri-footprint database (Blonk Agri-footprint BV, 2015a,b). The environmental impact data for the different transport modes are taken from the same database. The cost figures for the different products, 
transportation and processing steps were obtained as part of an extensive data collection from the literature and other sources. In case cost figures were not readily available, the data was calculated based on information from other sources such as labour and energy requirements and the country specific labour and energy costs.

\subsubsection{Scenarios}

Using the data described above, the model is tested for different scenarios that are then compared with respect to their results. Further specifications of the individual scenarios are detailed in the following.

- Base Case: The demand for different food groups $(\alpha=1)$ is taken from current daily consumption data based on the Dutch dietary consumption survey (Van Rossum et al., 2016) and scaled to the population level. The model is optimised for cost which seems to be most aligned with current consumer choice, thus representing a kind of status quo. Dietary supplements in the form of vitamin B12 supplements are not considered in this case, as no demand for this food category is included. The base case presents a reference case for the other scenarios and will be the basis for comparison.

- Status Quo Scenarios: Given the same setting as in the base case the model is optimised also with respect to different environmental objectives: climate change, land use, water use and fossil fuel depletion.

- Supplement Scenarios: In these scenarios the demand is no longer expressed in the form of food groups but in the form of a nutrient demand $(\alpha=0)$. Therefore, the food category demand $D_{k i}$ of the base case is converted via data on nutrient content $\left(a_{n p}\right)$ to obtain the nutrient demand $d_{i n}$. Vitamin B12 supplementation is possible to provide the required amount while palatability constraints in relation to portion sizes are added to ensure acceptability and feasibility of the dietary consumption. Provided that a plant-based diet requires a different dietary consumption, portion sizes are assumed to be higher than current standard portion sizes for plant-based food products. Given these specifications, the model is optimised for the different objectives: cost, climate change, land use, water use and fossil fuel depletion.

Furthermore, a multi-objective analysis is carried out using compromise programming (Zeleny, 1973) for the different demand scenarios and the epsilon-method (Ehrgott, 2009) for selected indicators in the case of nutrient demand. 


\subsection{Numerical Analysis}

\subsubsection{Optimising for single objectives}

Using Xpress-IVE version 7.2., the model is solved for the different scenarios with respect to the aforementioned economic and environmental objectives, minimising a single objective at a time. As a result, different optimal solutions for the food system are obtained in connection with the different scenarios and the findings are compared in the following. Note, that in the following, nutrient demand scenarios are indicated with an asterix $(*)$ in tables and texts.

Table 2.4: Payoff Tables for product and nutrient demand scenarios

\begin{tabular}{|c|c|c|c|c|c|c|c|c|c|c|}
\hline & \multirow{2}{*}{$\begin{array}{c}\text { Base Case } \\
\text { TC }\end{array}$} & \multicolumn{4}{|c|}{ Status Quo } & \multicolumn{5}{|c|}{ Supplement Scenarios } \\
\hline & & $\mathrm{CC}$ & LU & WU & FD & $\mathrm{TC}^{*}$ & $\mathrm{CC}^{*}$ & $\mathrm{LU}^{*}$ & $\mathrm{WU}^{*}$ & FD* $^{*}$ \\
\hline Total Cost & 8953636 & $126 \%$ & $129 \%$ & $121 \%$ & $120 \%$ & $25 \%$ & $70 \%$ & $40 \%$ & $134 \%$ & $55 \%$ \\
\hline Climate Change $\left(\mathrm{kg} \mathrm{CO} \mathrm{CO}_{2}\right.$ eq) & 100358786 & $48 \%$ & $50 \%$ & $50 \%$ & $99 \%$ & $9 \%$ & $6 \%$ & $8 \%$ & $13 \%$ & $39 \%$ \\
\hline Land Use (m2a) & 54135964 & $78 \%$ & $71 \%$ & $75 \%$ & $88 \%$ & $28 \%$ & $45 \%$ & $13 \%$ & $97 \%$ & $35 \%$ \\
\hline Water Use (m3) & 380568 & $213 \%$ & $102 \%$ & $31 \%$ & $190 \%$ & $114 \%$ & $10 \%$ & $95 \%$ & $5 \%$ & $75 \%$ \\
\hline Fossil Depletion (kg oil eq) & 2200016 & $97 \%$ & $127 \%$ & $112 \%$ & $86 \%$ & $93 \%$ & $51 \%$ & $89 \%$ & $83 \%$ & $27 \%$ \\
\hline
\end{tabular}

In this context, Table 2.4 presents the cost and environmental impact values associated to the Base Case, while the cost and environmental impact of the optimal solutions for all other scenarios are expressed in percentages of the Base Case values. The optimised objective for each scenario is shown underlined and in bold. Comparing the scenarios, with respect to their total cost and environmental impact, using the Base case as a reference, water use shows the biggest improvement potential with $31 \%$ of the Base Case value for the Status Quo and 5\% for the Supplement Scenarios. Fossil fuel depletion in contrast shows the smallest potential of improvement with values at $86 \%$ and $27 \%$ of the Base Case for the Status Quo and Supplement Scenarios. The Supplement scenarios generally have a higher potential to lower environmental impact. Moreover, the percentages shown in Table 2.4 indicate a shifting of burdens between the individual environmental impact indicators. For the Status Quo scenarios, this means for instance, that water use increases to $190 \%$ when fossil fuel depletion is minimised. In the case of the minimisation of climate change, water use is even higher, increasing to $213 \%$ in comparison to the Base case.

Figure 2.3 further illustrates these findings, while also showing the contribution of the three main phases, i.e. agriculture, processing and transportation, to the overall impact values. It can be seen that within the food system, the main contributor to cost and the different environmental impact categories is in most cases the agricultural production phase, followed by the processing phase, while transport plays a minor role for most scenarios. 


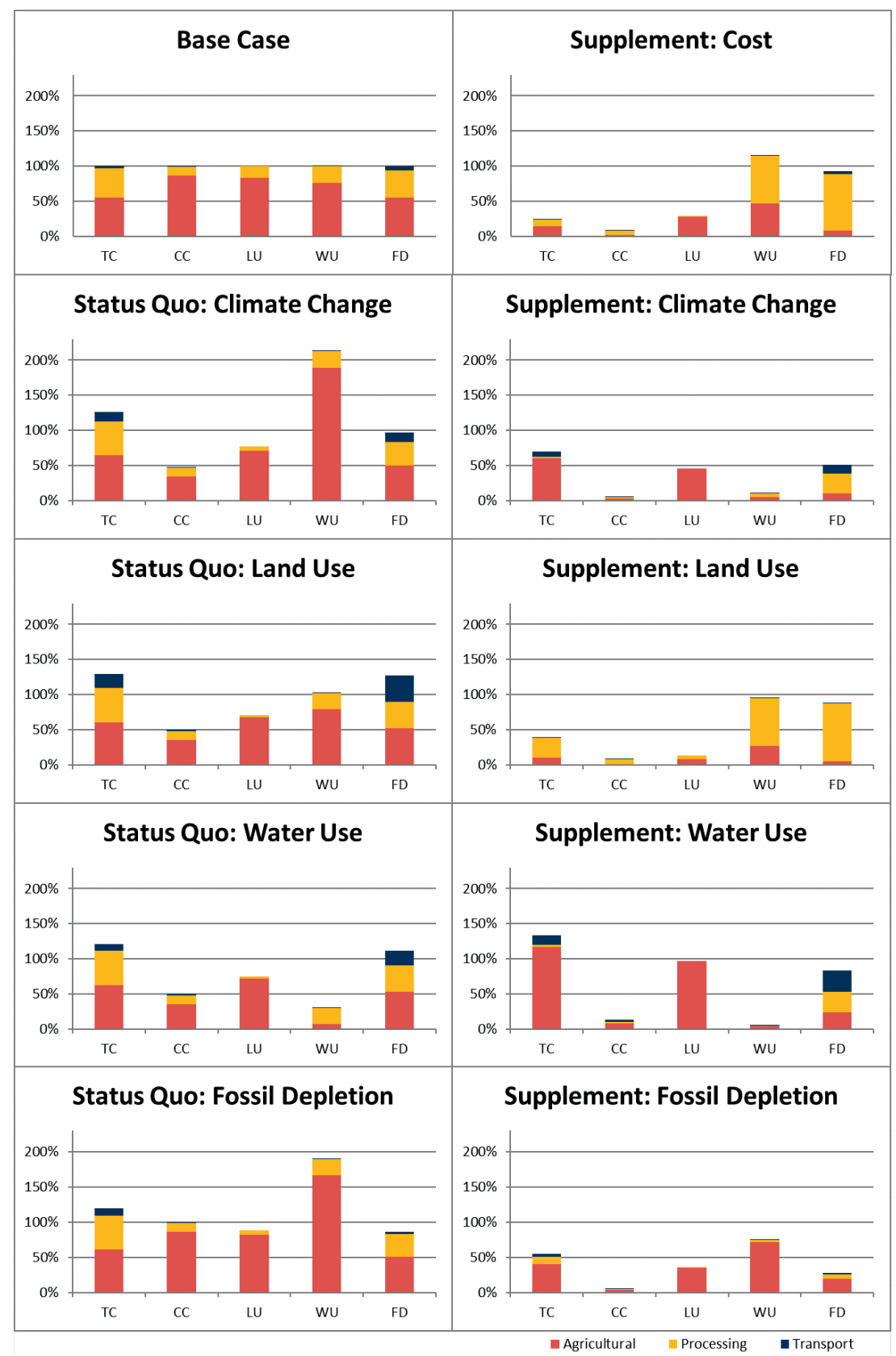

Figure 2.3: Impact per scenario for total cost (TC), climate change (CC), land use (LU), water use (WU) and fossil fuel depletion (FD), optimising over the different objective functions (in percentage of the base case) 
The impact of each phase within the system varies however, so that the processing phase gains in importance and plays a key role for the minimisation of cost and land use objectives in the Supplement scenarios. As transport mainly impacts climate change and fossil fuel depletion and has no or only little impact on land and water use, a higher contribution to fossil fuel depletion can be seen for scenarios in which land or water use are being optimised.

Figure 2.4 presents the impact value contribution of the countries selected as production and processing locations specified for each scenario. Note, that all values are given in percentages of the total impact values obtained in the Base Case (as shown in Table 2.4 ), values related to transportation are not assigned to a specific country and thus not included in this analysis.

Comparing the results for the different scenarios, the selected countries and their contribution to a specific impact indicator differ. For climate change for example, Indonesia is the biggest contributor in the Base Case, the FD and FD* scenario, while Malaysia is the main contributor to climate change in the Status Quo scenarios that optimise climate change (CC), land use (LU) and water use (WU). Comparing between impact indicators, it can be seen that the contribution of a country within a scenario varies per indicator. An example of this is France in the CC scenario, contributing a large share to water use but only little to all the other impact indicators. Another example is the $\mathrm{LU}^{*}$ scenario, where the Netherlands accounts for most of the impact associated to the different indicators with the exception of land use, for which Belgium makes the most prominent contribution. This means that depending on the scenario a country might be more or less affected by the different environmental pressures, which can be denoted as a shift of burdens between countries. In addition to this, the figure shows that for many scenarios, most of the impact associated to a specific indicator can be attributed to only one or two countries, while the rest of the countries make just minor contributions, thus resulting in an uneven distribution of the pressures among the different countries. More generally, comparing the results with respect to product and nutrient demand, Figure 2.4 again highlights the bigger improvement potential in the supplement scenarios (with nutrient demand), while also depicting a general trend for these scenarios towards smaller systems, consisting of fewer countries for production and processing locations. This is linked to the number of product types within the system associated to the different scenarios. 


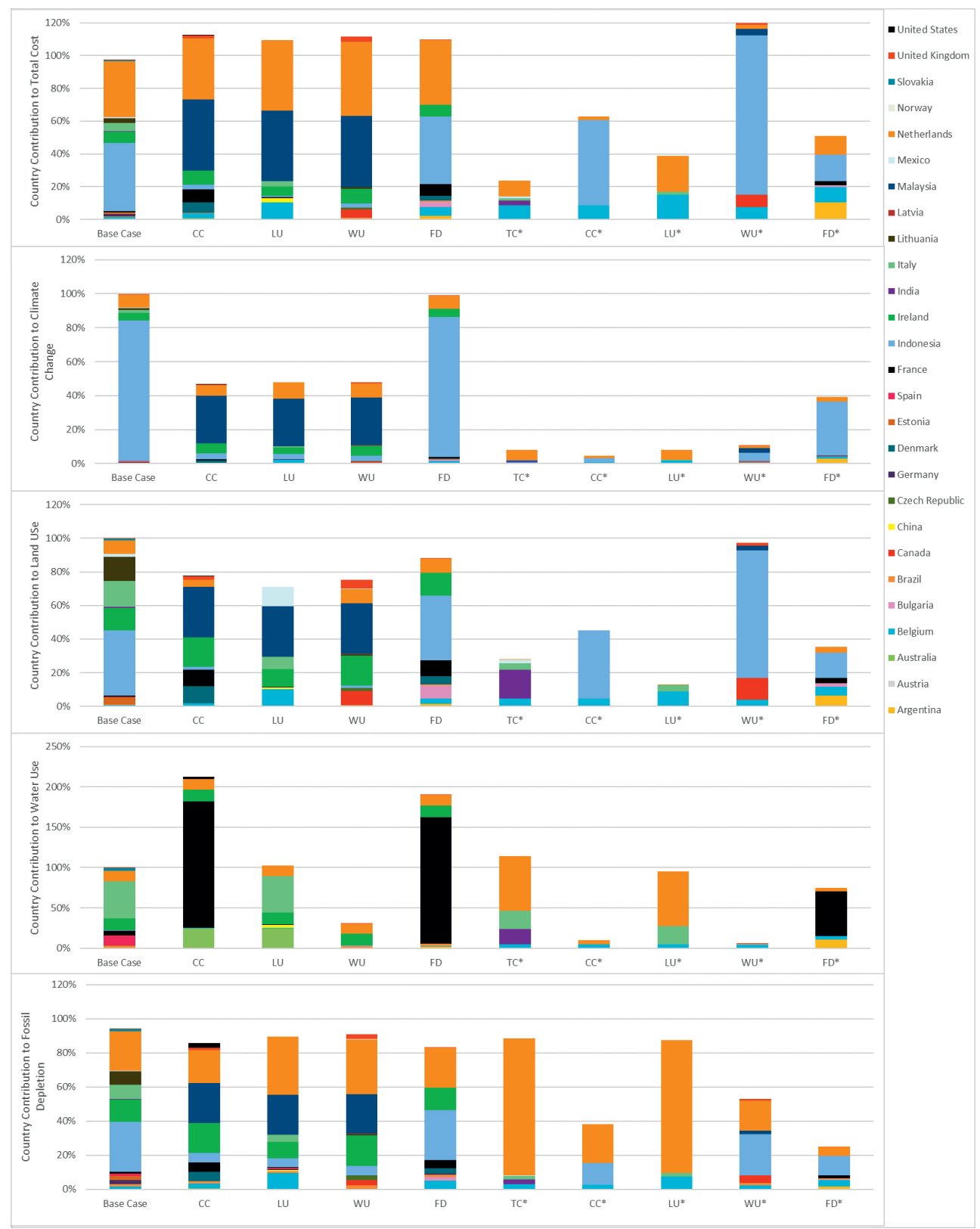

Figure 2.4: Impact contribution per country specified for each scenario 
In this context, Table 2.5 presents the total number of product types for each scenario, distinguishing between consumed products, resources and unused side-streams within the system. From this comparison, it can be seen, that the total number of product types is considerably higher for scenarios including beef and/or dairy products (all product demand scenarios and FD* (see Table 2.6)) than for scenarios with plant-based consumption, requiring significantly less resources. In comparison to the number of products consumed, the number of resources is about 5 times the number of product types for the Base Case and Status Quo scenarios and for minimisation of fossil fuel depletion in the Supplement scenarios. Furthermore, due to the relationships and conversion between product types, there are scenarios in which not all product types are fully used or side streams of products are wasted.

Table 2.5: Total products within the system categorised by usage

\begin{tabular}{lcrrrrrrr}
\hline & Base Case & \multicolumn{3}{c}{ Status Quo } & \multicolumn{5}{c}{ Supplement Scenarios } \\
\cline { 2 - 9 } & Cost & CC/FD & LU/WU & TC* & CC $^{*}$ & LU* $^{*}$ & WU* $^{*}$ & FD* \\
\hline Consumed & 9 & 10 & 10 & 5 & 3 & 5 & 4 & 5 \\
Resources: & 49 & 50 & 49 & 5 & 3 & 6 & 14 & 31 \\
- Processed (no waste) & 43 & 44 & 43 & 5 & 3 & 6 & 14 & 28 \\
- Processed (partly wasted) & 6 & 6 & 6 & & & & & 3 \\
Unused side-stream & & & & & & & 4 & 5 \\
\hline Total Produced & $\mathbf{5 8}$ & $\mathbf{6 0}$ & $\mathbf{5 9}$ & $\mathbf{1 0}$ & $\mathbf{6}$ & $\mathbf{1 1}$ & $\mathbf{2 2}$ & $\mathbf{4 1}$ \\
\hline
\end{tabular}

Moreover, for the Status Quo scenarios, only two product compositions are observed, one for the scenarios $\mathrm{CC}$ and FD and one for the scenarios $\mathrm{LU}$ and WU, while for the supplement scenarios product composition experiences more flexibility. Investigating the product mix for consumption in greater detail, Table 2.6 emphasises the limited choice of product mixes for the scenarios with product demand. The table further highlights that the three product mixes observed in the case of product demand, only show minor differences in their composition, such as for example a switch from rye bread to wholemeal bread (CC/FD to LU/WU). Given the differences in the impact values for the different scenarios (outlined in Table 2.4), these findings indicate the importance of the underlying production system configuration and its impact on the sustainability indicators for the product demand scenarios. The supplement scenarios in contrast allow for more flexibility due to the nutrient demand and therefore showcase a wider variety of product mixes, that clearly differ from the product demand scenarios. 
Table 2.6: Product mix consumed

\begin{tabular}{|c|c|c|c|c|c|c|c|c|}
\hline \multirow[b]{2}{*}{ Product (in kg) } & \multirow{2}{*}{$\begin{array}{c}\text { Base Case } \\
\mathrm{TC}\end{array}$} & \multicolumn{2}{|c|}{ Status Quo } & \multicolumn{5}{|c|}{ Supplement Scenarios } \\
\hline & & $\mathrm{CC} / \mathrm{FD}$ & LU/WU & TC* & $\mathrm{CC}^{*}$ & LU* & $\mathrm{WU}^{*}$ & FD* \\
\hline Milk, raw & 5964000 & & & & & & & \\
\hline Cream, full & & 148506 & 148506 & & & & & \\
\hline Cream, skimmed & & & & & & & & 170538 \\
\hline Milk, full & & 5815494 & 5815494 & & & & & \\
\hline Milk, skimmed & & & & & & & & 2134031 \\
\hline Beef (from dairy system) & 52526 & 52317 & 52317 & & & & & \\
\hline Veal & 6988 & 6960 & 6960 & & & & & \\
\hline Beef (from meat cattle) & 175686 & 175923 & 175923 & & & & & \\
\hline Soybean drink & & & & 10080000 & & 10080000 & & \\
\hline Spinach, fresh & 352800 & 352800 & 352800 & & & & 4386134 & 5040000 \\
\hline Frozen Spinach (in Plastic) & & & & 5040000 & 5040000 & 5040000 & & \\
\hline Beans, dry canned & & 67200 & 67200 & & & & & \\
\hline Chickpeas,canned & 67200 & & & 2249081 & & & & \\
\hline Peanuts, without shell & 84000 & 84000 & 84000 & 152028 & 4039001 & & 5040000 & 726808 \\
\hline Peanut butter & 67200 & 67200 & 67200 & & & & 2520000 & \\
\hline Rye Bread & 2200800 & 2200800 & & & & & & \\
\hline White Bread & & & & & & 2710387 & & \\
\hline Wholemeal bread & & & 2200800 & & & 783024 & & \\
\hline Vitamin B12 Supplements & & & & 5.67 & 5.67 & 5.67 & 5.67 & 3.8 \\
\hline
\end{tabular}

\subsubsection{Heterogeneous demand}

While the potential impact reduction is significantly bigger for the supplement scenarios, the shift from the current consumption mix in the product demand scenarios to the consumption mixes in the supplement scenarios might not be easily accepted in practice, finding resistance in the population. Thus, it is interesting to investigate the economic and environmental impact in the presence of heterogeneous demand scenarios allowing for a more gradual change, instead of considering consumer behaviour to be homogeneous, where either food groups or nutrients are demanded. Heterogeneity is modelled by varying the share of $\alpha$ in constraints (2.7) from 0 to 1 , with 0 representing the nutrient demand scenarios and 1 the food category demand scenarios. Figure 2.5 presents the objective value change related to a change in $\alpha$ (x-axis) for each of the objectives. Note, that all values are denoted in percentages of the impact values obtained in the Base Case. The results presented in the figure show a smooth and continuous change in the assessed criteria, where the impact for each objective increases gradually with $\alpha$. These results again emphasise that the improvement potential is biggest for the scenarios with homogeneous nutrient demand, while also showing that even minor changes in the demand can help to decrease the impact associated with the human diet. 


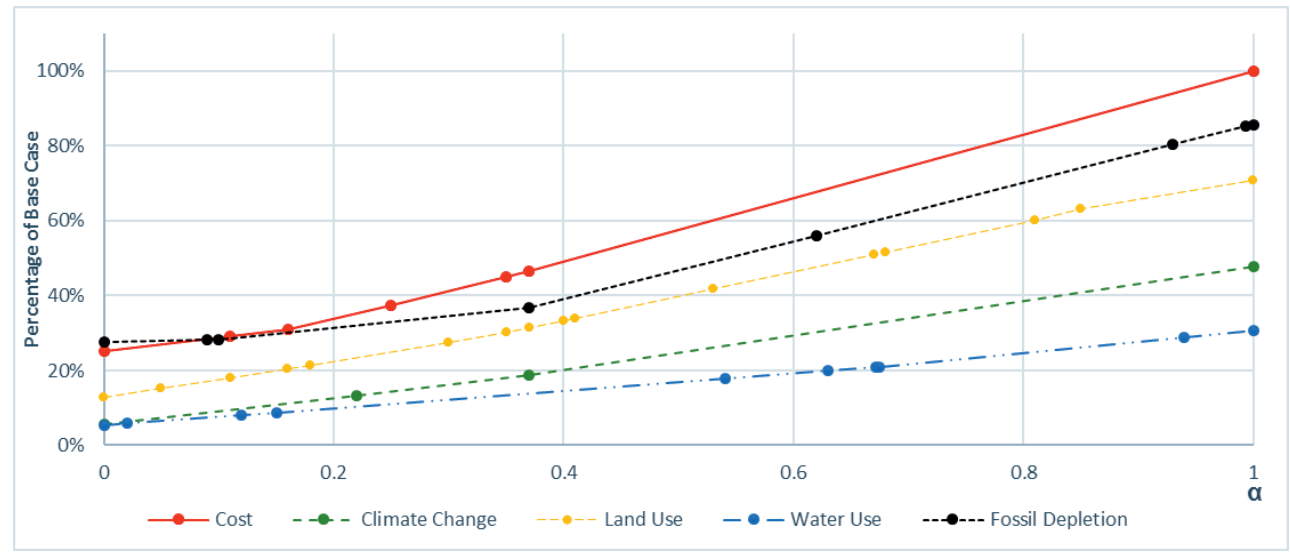

Figure 2.5: Objective values as a function of the share of food category demand $(\alpha)$ in percentage of the Base Case

\subsubsection{Bi-objective optimisation: $\epsilon$ - constraint method}

Given the conflicting nature of the different objectives and the trade-offs between them, a bi-objective analysis is carried out deriving sets of Pareto optimal solutions using the $\epsilon$-constraint method. A detailed description of the method as well as other approaches for multi-criteria opti-misation can be found in Ehrgott (2009).

Two bi-objective analyses are carried out for the case of nutrient demand to determine the relationships between cost and climate change as well as water use and climate change. The analyses therefore investigate one relation between an economic objective (i.e. cost) and an environmental objective (i.e. climate change) and one relation between different environmental objectives (i.e. climate change and water use). Climate change was chosen for investigation as it is the most widely used environmental objective in the scientific literature, whereas water use represents a predominantly local impact indicator in contrast to the global impact of climate change. However, other interesting relationships might exist for other indicators, such as a potential conflict between land use and cost or land use and water use. The efficient solutions for both bi-objective analyses are calculated by minimising one of the objectives while the other objective constrains the problem, the process is iterated for the different ranges obtained from a sensitivity analysis with respect to the right-hand side of the constraint. Thus the number of iterations depends on the analysed scenario. The obtained sets of efficient solutions for the cases presented here are depicted in Figure 2.6. 


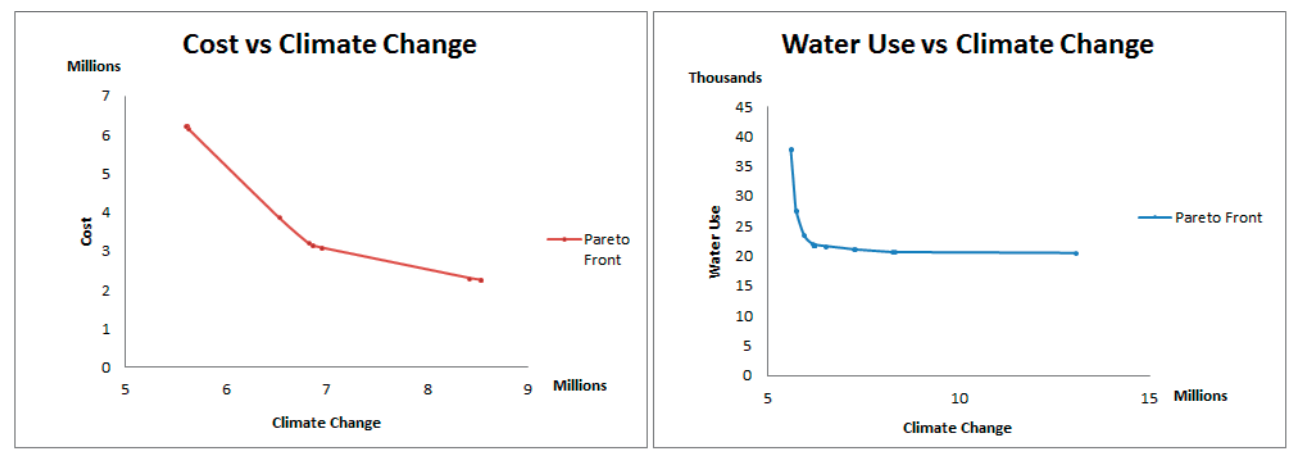

Figure 2.6: Trade-off curves for multi-objective optimisation in the context of the supplement scenarios

For the analysis based on cost and climate change, 15 iterations were needed to obtain the set of Pareto optimal solutions. The graph features a distinctive kink in the middle of the trade-off curve, dividing the curve into two line sections. The solutions to the left of the kink cohesively contain a smaller number of product types than the solutions to the right of the kink as the number of product types consumed generally decreases for more environmental friendly solutions. The different slopes of the two line sections indicate that climate change reductions along the first section are more costly, whereas reductions in the second section require a smaller increase in cost. The changes in the solutions along a particular line section are mainly caused by changes in production location. The best solution depends on the decision maker's preferences, with the two end points representing more extreme preferences while the kink in the middle depicts a more balanced solution. For the analysis based on water use and climate change, a total of 19 iterations were conducted to derive the set of Pareto efficient solutions. The trade-off curve in this case adopts a smoother curve, starting with a steep decline and merging into an almost flat continuation. In the flat part of the curve big improvements can be made with respect to climate change for a small increase in water use. This is mainly caused by changes in the composition of the product mix. Seven of the efficient solutions on this line segment include dairy products in the product mix, however the quantity of dairy products decreases for solutions with lower climate change impact. In the steep part of the curve, large improvements in water use can be obtained by relatively small increases in climate change. Shifts are caused by both, small changes in product mix composition as well as the choice of production locations. 


\subsubsection{Multi-objective optimisation: Compromise programming}

While the bi-objective approach using the $\epsilon$-constraint method provides interesting insights regarding the trade-offs between two different objectives, it does not take all the objectives into account. Given that all objectives are considered of equal importance, it is interesting to consider the relationships between all the objectives using a multi-objective approach. Compromise programming (CP), first developed by Zeleny (1973), is a useful tool in this context, allowing the decision maker to find compromise solutions, that are as close as possible to the desired solutions for each of the conflicting objectives. The basic idea of CP is thus to first establish the "ideal point" for each of the objectives $j$, i.e. the optimal value associated with each single objective, and then to minimise the distance between these ideal points and the compromise solution. Given that the units are often different between indicators, distances are normalised using the distance between the ideal point and a pessimistic point as a reference. The degree of closeness $d_{j}$ is then defined by

$$
d_{j}=\frac{Z_{j}^{*}-Z_{j}(x)}{Z_{j}^{*}-Z_{* j}}
$$

where $Z_{j}^{*}$ refers to the ideal point, $Z_{j}(x)$ refers to the point under consideration and $Z_{* j}$ is the pessimistic point. Note, due to the complexity of identifying the actual worst point associated with an indicator, the pessimistic point is estimated by the worst solution found for an indicator when optimising for single objectives. The distances between solutions and the ideal point is measured using the following set of distance functions:

$$
L_{p}=\left[\sum_{j=1}\left(d_{j}\right)^{p}\right]^{1 / p}
$$

The $L_{1}$ and $L_{\infty}$ metric, providing the bounds of the compromise set (Yu, 1973), can be obtained using the following formulations:

$$
\operatorname{Min} L_{1}=\sum_{j=1} \frac{Z_{j}^{*}-Z_{j}(x)}{Z_{j}^{*}-Z_{* j}} \quad \text { s.t. } \quad x \in X
$$

and

$$
\operatorname{Min} L_{\infty}=D_{\max } \quad \text { s.t. } \quad \frac{Z_{j}^{*}-Z_{j}(x)}{Z_{j}^{*}-Z_{* j}} \leq D_{\max } \quad \forall j \in J \quad x \in X
$$


The extended goal programming model of Romero (2001) given by the following formulation

$$
L_{e}=(1-\lambda) D_{\max }+\lambda \sum_{j=1} d_{j}
$$

is applied to find solutions, taking into account both efficiency ( $L_{1}$ metric) and equity ( $L_{\infty}$ metric) considerations, with $\lambda$ being a control parameter, regulating the bias towards efficiency of the solution. The analysis is carried out in the context of both product and nutrient demand.

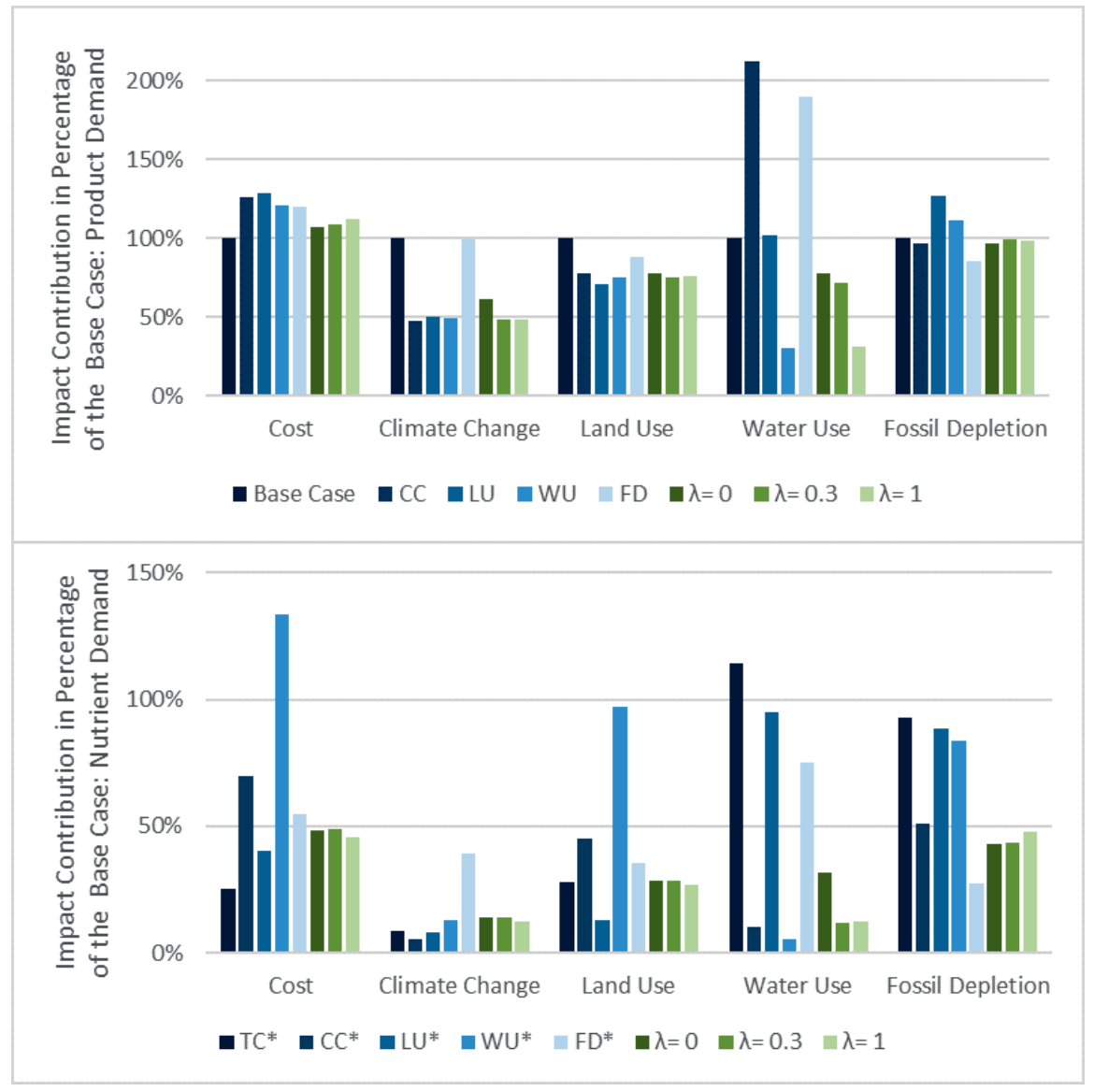

Figure 2.7: Impact comparison between scenario and compromise solutions for both product and nutrient demand in percentage of the Base Case 
Results of the analysis are shown in Figure 2.7, comparing the compromise solutions (represented in green) to the solutions found for the single objective optimisation (represented in blue). The depicted compromise solutions are $L_{1}(\lambda=1), L_{\infty}(\lambda=0)$ and an intermediate solution $L_{e}$ with $\lambda=0.3$. The figure shows, that CP provides intermediate values in the lower ranges of each indicator, resulting in overall more balanced solutions. Moreover, the different compromise solutions show similar results for the individual impact indicators with the exception of water use, where the difference between the $L_{1}$ and $L_{\infty}$ solution is more prominent.

\subsection{Discussion and Conclusion}

This chapter proposes an integrated modelling approach for the global food system under nutritional considerations, optimising over both cost and environmental objectives. To the best of our knowledge this research presents the first (multi-objective) network design model for a holistic food system in which consumption and production decisions are incorporated in a common framework.

Furthermore, the applicability of the model is illustrated based on a real life case study and tested for different scenarios. The scenarios investigated in this research can be grouped in product demand scenarios and scenarios with a nutrient demand. Within a group the scenarios differ with respect to their objective function, optimising cost and various environmental indicators, namely climate change, land use, water use and fossil fuel depletion. Product demand scenarios, consisting of the Base Case and Status Quo scenarios, depict the current consumption patterns in the Dutch society, whereas the scenarios with a nutrient demand (Supplement Scenarios) describe possible future what-if scenarios. Given the size of the system under consideration, as well as its holistic nature and the various aspects included in the model and numerical analysis, the findings of this research are manifold.

While the chapter investigates supply chain activities, with respect to three different phases in the food system, i.e. agricultural production, processing and transportation, the findings show that agricultural production is the main contributor in the system. This is in line with other scientific research (Weber and Matthews, 2008; Garnett, 2011) and has the effect that the focus in this discussion will be predominantly on findings related to production aspects.

Viewing the food system as a whole, the findings show, that consumption patterns impact the size and configuration of the underlying system. Plant-based consumption generally requires less resource input from other supply chains and hence results in simplified systems with less intermediary steps and greater transparency. Animal systems on the other hand require extensive resource input in the form of feed and 
often comprise of several processing steps, adding to the complexity of the system. Considering the different phases in the system, the impact per phase varies depending on the objective function and for the different environmental impact indicators. This can be attributed to the fact that the different phases in the system have different environmental profiles, contributing differently to the individual indicators. In most cases however, the agricultural production phase is predominant, contributing most to cost and environmental impact, followed by the processing phase, whereas the impact of transportation is of minor importance in these kind of systems. Measures aimed at improving cost and the environmental impact of the system, such as the development of technological advancements or new production methods, should be targeted accordingly to maximise their potential.

The findings with respect to the product mix, show a higher product diversification in the scenarios with product demand, whereas the nutrient demand offers more flexibility and thus results in fewer product types consumed with greater variation between scenarios. The observed optimal product mixes for the different scenarios and their contribution to different environmental impact indicators, confirm the findings of other researchers, that a shift towards a more plant-based dietary consumption holds the greatest potential to reduce the environmental burden (Hallström et al., 2015). For most objective functions in the Supplement scenarios, with the exception of the minimisation of fossil fuel depletion, the results even suggest a shift to a fully plant-based dietary consumption. This is, however, only possible due to the vitamin B12 supplementation. In this context, it should also be noted, that while it holds the greatest potential to reduce the environmental burden, a fully plant-based dietary consumption, requires significant changes in the composition of the diet. Thus, it might be less accepted by the population, even though it is feasible to supply the nutrient demand with the plant-products and supplements included in this research. The analysis considering heterogeneous demand scenarios shows in this context however, that even small changes in our dietary consumption can help to decrease the environmental impact associated with our diet. For future research, the inclusion of other product types, such as meat substitutes, in combination with more advanced palatability constraints could make the results more widely acceptable and realistic. In addition to this, it could be of interest to investigate government and other stakeholder measures that can influence demand shifts in combination with social aspects.

The findings further depict a shifting of burdens between the individual indicators due to existing trade-offs. This means, that the minimisation of one indicator can lead to a substantial increase of another indicator and thus can have a tremendous effect on a different aspect of the environment.

The bi-objective approach, using the $\epsilon$-constraint method, provides a way to investigate these trade-offs and obtain a set of efficient solutions, while the multi-objective approach 
using compromise programming provides more balanced solutions dealing with multiple conflicting objectives, by considerably improving the environmental factors while only moderately increasing costs. Based on these findings, the decision maker can choose the best solution based on his or her preferences.

Moreover, a shifting of burdens can also be observed between countries (i.e. the production locations), as the contribution of a country differs depending on the scenario, as well as for cost and the individual environmental indicators. Thus, the global optimal solution might result in an increase in the burden from the local perspective of certain countries. This raises two issues. Firstly, in the context of predominantly local impact factors, such as water or land use, an increase in a country where water or available land is scarce, can be more severe and harmful for the environment than in other countries. This should be taken into account in the decision making progress and investigated further in future research by for example including capacities. Secondly, for global impact factors, such as climate change, it is important to raise awareness of the global nature of the problem and encourage international collaboration as a shift between countries can lead to an overall reduction. This outsourcing of emissions on the other hand questions the validity of current emission schemes and requires policies that penalise and compensate in a fair manner.

While there are valuable insights to gain from this study, it is important to note that it has its limitations and that some of the underlying assumptions require further attention. One of the main limitations in this study are the system boundaries and the amount of products, processing options and production locations considered, as in reality the amount of options in the food system is significantly bigger, whereas this study relies on a limited number of products. It should be noted in this context, that the results and optimal solutions obtained are product dependent and thus are likely to change with different product input. Furthermore, in practice, the relation between different products, such as resource requirements and by-product ratios, are not inevitably fixed but depend on prices and other factors in the system, such as for example availability and quality aspects. This holds especially true for the composition of feed and should be investigated further in future research.

Another important limitation in the context of the food system is that in reality there are more actors and demand locations in the system, than considered here. Every country has a demand and supply for food products and given the limited resources there is competition between countries. Countries have production capacities and an interest to maintain some level of self-sufficiency and not become too dependent on the supply of other countries. Seasonality plays an important role in relation to the production of food products within a country and the availability of food products is subject to uncertainty due to weather conditions and other external influences.

In addition to this, the number of nutrients included in the model only represents a 
small part of the human diet and nutritional requirements. A shift from animal products towards a more plant-based dietary consumption is likely to affect the nutritional intake in multiple ways and not just limited to the nutrients considered here. For future research, it could thus be of interest to examine the effects of this shift in a broader dietary setting, taking into account a more complete nutritional profile.

While social sustainability is here represented in the form of health considerations, i.e. the nutritional intake, there are many other aspects that are of relevance from a social perspective, such as fair trade, animal welfare and employment aspects. Most of these aspects are however difficult to aggregate, quantify and measure in a global context where numerous different social systems converge and interact.

This model and its findings rely on a substantial amount of data and while an extensive amount of data was gathered from LCA studies and a variety of literature sources a number of assumptions had to be made that might impact the results of the model. The data quality might therefore vary for different countries, processing and product types. Moreover, whereas this study assumes all data to be deterministic, this is hardly viable in practice due to uncertainties. The model could thus be extended using stochastic or robust approaches in order to account for such uncertainties in the data. 


\section{Chapter 3}

\section{Network Perspective}

\section{A nutritional comparison and production \\ perspective: Reducing the environmental footprint of the future}

This chapter is published as:

Rohmer, S. U. K., Gerdessen, J. C., Claassen, G. D. H., Bloemhof, J. M., \& van't Veer, P. (2018). A nutritional comparison and production perspective: Reducing the environmental footprint of the future. Journal of Cleaner Production, 196, 1407-1417. 


\section{Abstract}

The current food system, with its consumption and production activities, threatens our environment and depletes the resources of future generations. Changing the food system, by consuming foods with lower environmental footprint and choosing more environmental friendly production and distribution alternatives, holds potential to reduce the environmental impact. The aim of this research is to propose healthy and sustainable alternatives to the current consumption of cow's meat and dairy products in the Netherlands, under consideration of the underlying production system. Thus, the study applies linear programming techniques to construct consumption alternatives, taking into account the underlying production and sourcing of products. In this context, different environmental objectives are investigated and compared, namely climate change, land use, water use and fossil fuel depletion. Comparisons are made between the different alternatives with respect to their effect on the overall dietary intake. Four consumption alternatives are proposed, varying with respect to their environmental footprint, food composition and underlying food system. The results show that shifting towards a more plant-based consumption holds both an improvement potential in terms of the environmental impact as well as benefits from a health perspective. Moreover, trade-offs exist between the different environmental indicators, and the choice of environmental objective impacts the solution with respect to the consumption and production of foods. The research demonstrates the importance of taking production relationships into account and shows, that it is possible to propose healthy and environmental friendly alternatives for the future. 


\subsection{Introduction}

The environmental footprint of the food system and the challenge of ensuring food security for future generations has received growing attention over recent years. Population growth and higher living standards are drivers of the rising demand for food and an excessive meat consumption, with predictions indicating a $70 \%$ increase in the demand for food by 2050 and a comparable rise in the consumption of meat by 2030 (Fiala, 2008; FAO, 2009). With today's food system accounting for about $20-30 \%$ of the global greenhouse gas emissions (Vermeulen et al., 2012), these predicted developments are likely to put further pressure on the environment in the form of global warming, resource depletion and the extinction of species (Garnett, 2013). Among the products with the highest impact, contributing to these environmental burdens, are beef and dairy (Notarnicola et al., 2012; Steinfeld et al., 2006).

Changing current dietary consumption patterns can thus lead to improvements in the environmental impact associated with the human diet and offset some of the environmental pressures faced by today's society. In this context, recent scientific research has increasingly focused on investigating the environmental impact of food items and different dietary alternatives in order to propose more sustainable consumption patterns. Such studies include comparisons of different dietary scenarios in terms of their environmental impact (Berners-Lee et al., 2012; Meier and Christen, 2012; van Dooren et al., 2014) and mathematical modelling approaches, in the form of diet models, that are applied in order to propose alternative dietary patterns to aid decision making in the nutritional context (MacDiarmid et al., 2012; Tyszler et al., 2014, 2016; Wilson et al., 2013). The general findings from the literature suggest that vegan and vegetarian scenarios hold the biggest potential to lower the environmental impact of human dietary consumption. Hallström et al. (2015) provide a systematic review related to dietary changes and the associated environmental impact, focusing on land use change and greenhouse gas (GHG) emissions. Reviewing 14 scientific articles, the research of Hallström et al. (2015) further shows that replacing meat and in particular ruminant meat can lead to significant improvements in terms of greenhouse gas emissions. Similar results are found with respect to land use change. Mertens et al. (2017) provide a review of different ways in which health aspects are incorporated in research on more environmentally sustainable diets with an increased focus on the health aspects related to the consumption changes.

While, it is possible to achieve good results from an environmental perspective, by reducing the amount of meat and dairy products in our diet, the effect on palatability, acceptance and nutritional intake should be taken into account when proposing alternative dietary scenarios (MacDiarmid et al., 2012). Beef and dairy products are important sources of vital nutrients such as protein, vitamin B12, iron, zinc and calcium. Eliminating or reducing these products in our diet without adequate substitution might thus 
result in nutrient deficiencies.

To the best of our knowledge, there are no studies that consider both the dietary aspects of the consumption of food and its impact on the underlying supply chain configurations within a common framework. Existing research on sustainable diets and dietary consumption alternatives, excludes aspects related to the production of food products and thus neglects the existing links and interrelations between products as well as other aspects influencing the environmental footprint of a product, such as for example processing and transportation (Van Mierlo et al., 2017). The environmental impact of food products, including meat and dairy products, is not solely defined by the product type but can vary significantly depending on the production system behind it, e.g. aspects related to the production and supply chain configuration. These aspects should thus be taken into account when proposing and evaluating dietary consumption alternatives, reducing the environmental impact associated with food consumption patterns.

The aim of this study is to propose alternative food consumption patterns with lower environmental impact, under consideration of the underlying food system, while ensuring nutrient equivalence for certain key nutrients, using linear programming techniques. The environmental impact of dietary alternatives is investigated for different environmental indicators, namely climate change, land use, water use and fossil fuel depletion. The research further analyses and compares the effect of the proposed alternatives on the overall dietary intake with respect to their nutrient contents.

\subsection{Materials and Methods}

This study develops a model for the transition from meat and dairy towards plant-based alternatives and substitution of these products while also incorporating the underlying food system. The underlying food system refers to the sourcing of products and comprises transportation and processing steps as well as the agricultural production phase related to specific food consumption patterns. Substituting only a subset of food items within the framework of the human diet, the model keeps energy intake constant and constrains a number of selected key nutrients in order to obtain an equivalent dietary contribution as the one provided by beef and dairy products. The resulting dietary consumption alternatives are then evaluated based on their nutrient contribution with respect to a number of other nutrients. In this context, Figure 3.1 presents the methodological framework of this study (dark grey triangle), in comparison to the traditional framework for diet modelling (light grey rectangle). 


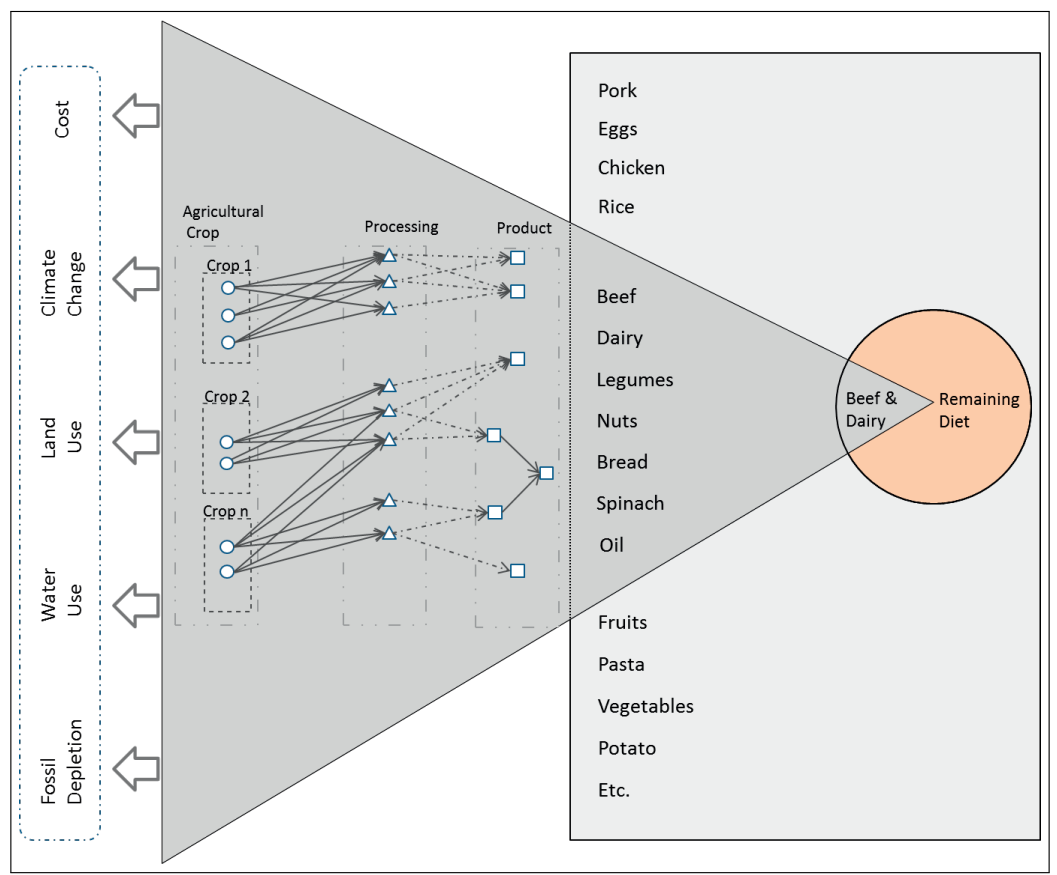

Figure 3.1: Methodological framework

It can be seen from the figure, that the new framework extends the analysis, by incorporating the underlying production system, at the cost of limiting the dietary analysis to a partial dietary assessment, while the traditional approach focuses on the overall dietary intake and the selection of food products but does not include any production aspects. Given the partial dietary assessment, the following provides more details on the selection of food items and nutrients considered as well as the chosen underlying food system.

\subsubsection{Selection of food items and nutrients considered}

Given the abundance of choice and alternatives in the context of the human diet and food production alternatives, the scope of this study will be limited to a selection of products, representing and contributing to a small but important part of our dietary needs. Focusing on sustainability, the selection of food products was guided by the environmental contribution of products. Thus, beef and dairy products, as the key contributors to the environmental pressures (Notarnicola et al., 2012; Steinfeld et al., 2006), are at the center of this research while a number of plant-based products, that are good sources in terms of the key nutrients present in beef and dairy, are selected as alternatives with lower 
environmental impact. Five key nutrients have been chosen in relation to meat and dairy and are included in the model: protein, iron, zinc, calcium and vitamin B12 (Bender, 1992). These key nutrients are constrained to be at least equivalent to the level provided by the amounts of beef and dairy currently consumed. Furthermore, energy intake is fixed by constraints in the model and thus kept constant for tall alternatives. Several other nutrients are included for evaluation purposes in order to allow for a more general comparison of the healthiness of the resulting partial dietary substitution by observing the ex post levels of these nutrients. This comparison is carried out with respect to the following nutrients: dietary fibre, saturated fatty acids, vitamins A, B1, B2, C, D, E and folate as well as potassium, magnesium and sodium.

The plant-based alternatives have been chosen based on their nutrient profile, with respect to the key nutrients, as well as their popularity and acceptance, with reference to current Dutch dietary consumption patterns. The following food groups have been identified as good sources for the key nutrients considered in this research: legumes, cereals, nuts and green leafy vegetables. Legumes and in particular brown beans, soy and chickpeas are chosen as plant-based alternatives due to their high iron, zinc and protein content. Cereal products such as different types of bread, as well as nuts, including peanuts, may also contribute a substantial part to the daily intake of the same nutrients while furthermore contributing to the calcium intake in the human diet. Green leafy vegetables, represented by spinach, constitute a good source of calcium and iron. Vitamin B12 is not available from plant-based sources and supplementation, as an artificial source of vitamin B12, is not allowed in our model. Thus, the only sources of vitamin B12 included in this study are beef and dairy products, resulting in a minimum requirement for these products. In total, the chosen food categories contain 23 dietary products and product variations. Table 3.1 provides a more detailed overview of the foods within the selected categories. 
Table 3.1: Overview of chosen food groups

\begin{tabular}{lll}
\hline Food Group & Selected Foods $(\mathrm{n}=23)$ & Key Nutrients present $^{1}$ \\
\hline Meat & $\begin{array}{l}\text { Dutch beef (from dairy cattle); } \\
\text { Irish beef (from beef cattle); veal }\end{array}$ & $\begin{array}{l}\text { Iron, Zinc, Protein, } \\
\text { Vitamin B12 }\end{array}$ \\
\hline Dairy & $\begin{array}{l}\text { cream (full/skimmed); } \\
\text { milk (full/skimmed) }\end{array}$ & Calcium, Vitamin B12 \\
\hline Green Leafy Vegetables & spinach (warehouse); spinach (frozen in cardboard); & Calcium, Iron \\
& spinach (frozen in plastic) & \\
\hline Legumes & soybean drink; beans (dry canned) & Iron, Zinc, Protein \\
& chickpeas (canned) & Calcium, Iron, Zinc \\
\hline Nuts & peanuts (without shell); peanuts (blanched) & Protein \\
\hline peanuts (roasted); peanut butter & Calcium, Iron, Zinc \\
& rye bread; white bread; wholemeal bread & Protein \\
\hline Oil ${ }^{2}$ & refined soybean oil; refined palm oil & - \\
\hline${ }^{1}$ based on Voedingscentrum $(2016)$ & \\
${ }^{2}$ only included as a side product in the underlying production system &
\end{tabular}

\subsubsection{The production system}

In relation to these 23 products suitable for human consumption, a number of other products are selected based on product input and resource requirements in the underlying production system. Examples of such resources are for example, grains and flour for the production of bread or different feed products in the meat and dairy system. The final system consists of 72 products, comprising both consumer products as well as the resources needed for production of these products. Furthermore, 39 product dependent sourcing/production locations are included, focusing on the most relevant countries for imports of products to the Netherlands for which data was available.

Seven different modes of transport are considered in the model, with geographical position and the infrastructure of a country determining whether a mode choice is available between locations. Provided that the different modes relate to different infrastructure, such as for example canal, road and rail networks, the distances between countries depend on the specific infrastructure and thus on the mode choice. Transportation distances within a country are assumed to be of minor importance in this research due to the global scale and defined to be the same for all countries and modes considered in the model. 


\subsubsection{Alternatives and linear programming model}

Based on the chosen products and associated consumption and production system, a base case and four alternatives are investigated and compared with one another within the framework of this research. The base case serves as a reference case, building the basis for comparison. It considers the consumption of beef and dairy fixed to the current level and minimises the underlying product and production (including transportation and processing) costs which is thought to be most in line with current consumption behaviour. The four alternatives in contrast suppose a nutrient intake for the 5 key nutrients that is at least equivalent to the intake of these nutrients in the base case, while allowing for more flexibility with respect to the consumption of specific food products. Moreover, the environmental alternatives differ with respect to the objective functions that are being optimised, four environmental impact indicators are considered, namely climate change, land use, water use and fossil fuel depletion. They also include additional constraints to ensure palatability and acceptability of the resulting food consumption patterns. These constraints provide upper bounds to the changes in consumption for plant-based products (e.g. not more than a $10 \%$ increase in consumption). Note that, a plant-based diet is expected to require different consumption patterns and thus consumption is assumed to be higher in the model than current consumption for plant-based products to maintain nutrient requirements. An overview of the specifications/characteristics of the base case and the four environmental alternatives is provided in Table 3.2.

Table 3.2: Overview of the base case and environmental alternatives

\begin{tabular}{cccc}
\hline & Restrictions & Objective Function & Impact on \\
\hline Base Case & Food Consumption & Cost & Sourcing \\
Alternative 1 & Energy \& Nutrient Intake & Climate Change (CC) & Diet \& Sourcing \\
Alternative 2 & $"$ & Land Use (LU) & $"$ \\
Alternative 3 & $"$ & Water Use (WU) & $"$ \\
Alternative 4 & $"$ & Fossil Fuel Depletion (FD) & $"$ \\
\hline
\end{tabular}

A linear programming model was developed and solved for the different alternatives using Xpress-IVE version 7.2. For each alternative, the optimisation minimises the objective associated to the particular alternative in compliance with a number of constraints. The constraints included in the model are related to consumer demand as well as to resource requirements and transportation in the system. The full description and mathematical formulation of the model, including the variables and parameters used, can be found in Chapter 2. 


\subsubsection{Data input}

The nutrient composition of the selected food products was obtained from the food composition database for the Netherlands (RIVM, 2013). The demand of the food groups in the base case was obtained from the Dutch food consumption survey (Van Rossum et al., 2016). An extensive data collection, gathering data from literature as well as other sources, was carried out with respect to data regarding transport, product and processing costs. For the cases in which cost values were not directly available, cost figures have been calculated based on other available data, such as for example labour or energy requirements and country specific labour and energy costs.

The environmental impact data for transportation, processing and agricultural production of products, with regards to climate change, land use, water use and fossil fuel depletion, has been retrieved from LCA data in the Agri-Footprint database (Blonk Agri-footprint BV, 2015a,b) and was calculated using the ReCiPe method (Goedkoop et al., 2009). The database also provides the reference for all the product relations and conversion factors, such as how much and what kind of feed is needed for the production of beef and dairy.

\subsection{Results and Discussion}

Solving the model for the different economic and environmental objectives, results in a number of different dietary alternatives, varying with respect to their food group composition and underlying production system. The following section will provide an overview of these different alternatives found by the model and in this context elaborate on the differences in food group composition and the underlying production system. Moreover, we will present and discuss the findings with regards to cost and environmental impact of the dietary alternatives as well as their nutritional adequacy.

\subsubsection{Composition of dietary alternatives}

Focusing on the composition of the dietary alternatives, Figure 2 gives an overview of the overall food group compositions as a percentage of their contribution to total energy intake. Three different food group compositions can be observed for the base case and the different alternatives, with Alternative 2, 3 and 4 featuring the exact same food group composition. 


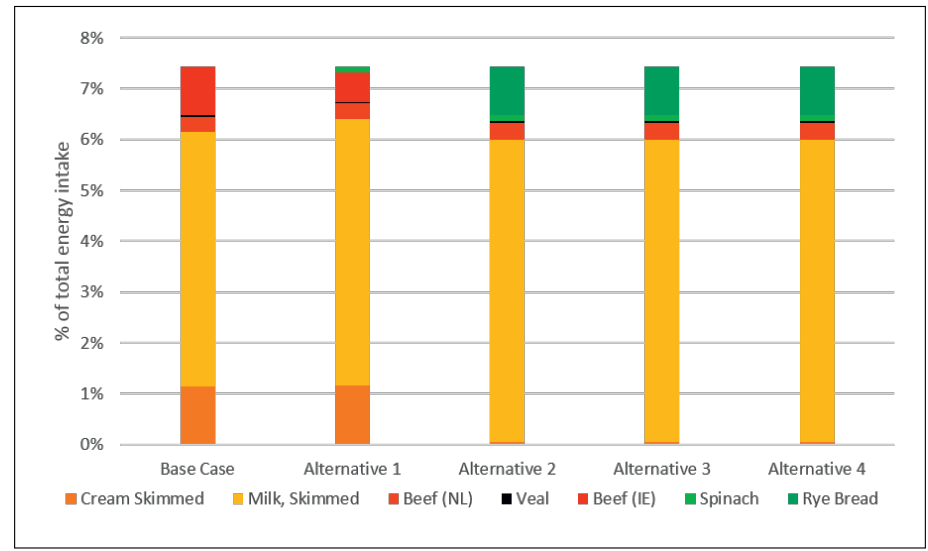

Figure 3.2: Food group composition of the base case and alternatives

Note, that while energy intake is kept constant for each alternative, the total amount of food consumed increases with more plant-based consumption, showing a $6 \%$ increase in food weight for the climate change alternative and a $13 \%$ increase for the land use, water use and fossil fuel depletion alternatives. In terms of energy intake, milk contributes the largest amount in all cases, however, while the base case is composed entirely out of meat and dairy products, the environmental alternatives also include spinach and in the case of the land use, water use and fossil fuel depletion alternatives also rye bread. Given these changes in the composition, it is possible to observe a shift towards more vegetarian/plant-based consumption alternatives, reducing the consumption of meat to $71 \%$ for the climate change objective and to $30 \%$ for all other optimised indicators (Table $3.3)$.

Table 3.3: Meat and dairy consumption in the base case and the four environmental alternatives

\begin{tabular}{|c|c|c|c|c|c|c|}
\hline & \multicolumn{2}{|c|}{ Base Case } & \multicolumn{4}{|c|}{ Consumption as compared to the base case (in 100\%) } \\
\hline & grams/day & energy contribution & $\begin{array}{c}\text { Alternative } 1 \\
(\mathrm{CC})\end{array}$ & $\begin{array}{c}\text { Alternative } 2 \\
(\mathrm{LU})\end{array}$ & $\begin{array}{c}\text { Alternative } 3 \\
(\mathrm{WU})\end{array}$ & $\begin{array}{c}\text { Alternative } 4 \\
(\mathrm{FD})\end{array}$ \\
\hline \multicolumn{7}{|l|}{ Meat products: } \\
\hline Cattle beef (IE) & $10.5 \mathrm{~g}$ & $1 \mathrm{en} \%$ & $60 \%$ & $0 \%$ & $0 \%$ & $0 \%$ \\
\hline Dairy beef (NL) & $3.1 \mathrm{~g}$ & $0.3 \mathrm{en} \%$ & $105 \%$ & $119 \%$ & $119 \%$ & $119 \%$ \\
\hline Veal (NL) & $0.4 \mathrm{~g}$ & $0.0 \mathrm{en} \%$ & $105 \%$ & $119 \%$ & $119 \%$ & $119 \%$ \\
\hline Cow's meat Total & $14.0 \mathrm{~g}$ & $1.3 \mathrm{en} \%$ & $71 \%$ & $30 \%$ & $30 \%$ & $30 \%$ \\
\hline \multicolumn{7}{|l|}{ Dairy Products: } \\
\hline Cream (skimmed) & $26.3 \mathrm{~g}$ & $1.2 \mathrm{en} \%$ & $101 \%$ & $5 \%$ & $5 \%$ & $5 \%$ \\
\hline Milk (skimmed) & $328.7 \mathrm{~g}$ & 5 en $\%$ & $105 \%$ & $119 \%$ & $119 \%$ & $119 \%$ \\
\hline
\end{tabular}


While overall meat consumption decreases, a slight increase in the consumption of meat from Dutch dairy farms, consisting of beef and veal, is observed for all alternatives. This is closely linked to a higher consumption of dairy. The reason for this is the relation between dairy and meat products from dairy farms, where production of one product is proportional to the production of the other products. The additional meat is thus a side product of the higher dairy production and incurs no additional cost from an economic or environmental perspective. A similar relation as between meat and dairy products, exists between dairy products, i.e. cream and milk. However, as cream is very energy dense and the energy intake is kept constant by the model, the model prefers to waste the additional amount of cream, resulting from an increase in milk production. Given the requirements in terms of energy and the key nutrients, the model is rather restricted with respect to finding different consumption alternatives. It is for example not possible to fully eliminate dairy and meat products from the consumption alternatives, due to the restrictions for maintaining vitamin B12 in the model. Bigger improvements from an environmental perspective, by shifting towards a fully plant-based consumption, without allowing supplementation is therefore not possible. From an environmental perspective it could thus be beneficial to include other animal products with lower environmental impacts, such as for example chicken and eggs, and/or investigate the use of dietary supplementation more thoroughly in future research.

\subsubsection{Changes in the underlying production system}

Changes in the underlying production system are observed for three different levels of the supply chain configuration; the product level, i.e. the type and quantity of products produced, the sourcing level, i.e. the production locations for these products, and the transportation level, i.e. the means of transport that are used within the production system. Due to the high complexity of the system and inter-linkages between the three levels, it is often difficult to clearly attribute an environmental impact improvement to a (single) change in one of the levels.

Table 3.4 shows, that at the product level, changes can be observed for the production of consumption products, which closely reflect changes in the dietary pattern, but also for the required resources and waste streams. 
Table 3.4: Total products within the system for each alternative categorised by usage

\begin{tabular}{lccccc}
\hline & Base Case & $\begin{array}{c}\text { Alternative 1 } \\
(\mathrm{CC})\end{array}$ & $\begin{array}{c}\text { Alternative 2 } \\
\text { (LU) }\end{array}$ & $\begin{array}{c}\text { Alternative 3 } \\
\text { (WU) }\end{array}$ & $\begin{array}{c}\text { Alternative 4 } \\
\text { (FD) }\end{array}$ \\
\hline Products for Consumption: & 5 & 6 & 6 & 6 & 6 \\
- Consumed (no waste) & 5 & 5 & 5 & 5 & 5 \\
- Consumed (partly wasted) & 0 & 1 & 1 & 1 & 1 \\
& & & & & \\
Resources: & 40 & 41 & 37 & 37 & 37 \\
- Processed (no waste) & 37 & 38 & 34 & 34 & 34 \\
- Processed (partly wasted) & 3 & 3 & 3 & 3 & 3 \\
Unused side-stream & 3 & 3 & 3 & 3 & 3 \\
\hline Total Produced & $\mathbf{4 5}$ & $\mathbf{4 7}$ & $\mathbf{4 3}$ & $\mathbf{4 3}$ & $\mathbf{4 3}$ \\
\hline
\end{tabular}

In addition to this, due to interrelations between products, eliminating or lowering the consumption of a product from our diet does not always mean that the production of the product goes down. This becomes apparent when looking for example at the relationship between milk and dairy beef; while milk consumption is generally attributed a lower environmental impact than beef, the production intrinsically ties the two together, making it impossible to produce one without the other. Making consumption decisions without considering these side streams might thus lead to unaccounted production that is wasted in the end, which results in understated environmental impact estimations.

At the sourcing level, the results from the model show, that sourcing locations vary depending on the dietary alternative. In this context, Figure 3.3 provides a geographical representation of the production systems for each of the optimised alternatives. The figure illustrates, that Alternative 2 for example only sources products from locations in Europe, Asia and South America, whereas Alternative 1 also includes Australia and Alternative 3 includes Canada as a sourcing location. Reasons for such variations are for example existing links between products and their production locations, as in the case of meat, where cattle farming in the Netherlands focuses predominantly on dairy production, while meat from beef cattle is imported from Ireland. Another reason for changes at the sourcing level are differences in the environmental performance with respect to a specific impact indicator related to the production of a product within a certain country. Such differences can be attributed to, for example, different production practices or differences in the energy mix used within a country. 


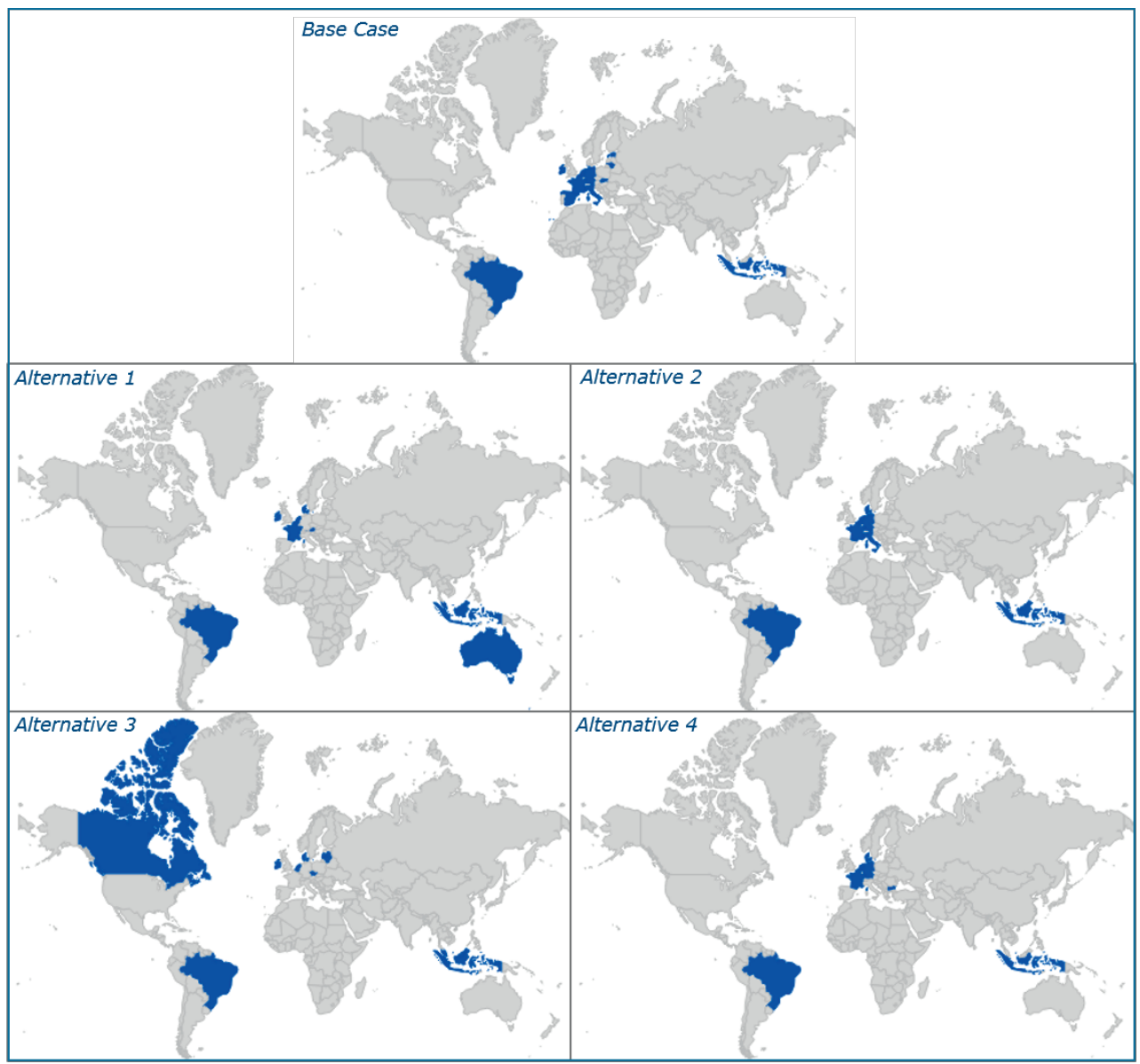

Figure 3.3: Geographical representation of the production systems for each of the dietary alternatives

Simply saying that the consumption of one product is better than the consumption of another is therefore not sufficient, due to significant variations in the environmental impact of a particular product. This has been for example shown in the scientific literature for the case of soy (Da Silva et al., 2010; Castanheira and Freire, 2013), which is a common resource for the production of animal feed.

Changes at the transportation level are often caused by changes in the sourcing of products, related to the availability of a transport mode. Referring back to the findings of Figure 3.3, it is for example not possible to use truck or train when shipping products from Canada or Australia to Europe while these might be the preferred modes of transport within Europe. In general, it should be noted, that many different alternative solutions 
for the sourcing and transportation phase exist, affecting the environmental impact values of the not optimised indicators. With respects to the results of this study it should be noted, that often there is more than one optimal solution. This is especially true when optimising land and water use, as transportation does not contribute to land use and only insignificantly to water use, therefore there is no difference between the available modes of transportation in terms of their contribution to the objective function and the model is free to choose sourcing locations without taking transportation modes and distances into account. Furthermore, the food consumption and production alternatives considered in this research are still limited, the actual food system is much more complex with countless links and interrelations between products and includes uncertainties with respect to for example the prices of products.

\subsubsection{Economic and environmental impact of the dietary alternatives}

Given the differences in the dietary composition as well as the underlying production system, the dietary alternatives differ with respect to their economic and environmental impact contribution. In this context, Figure 3.4 shows the impact of the different dietary alternatives with respect to cost and the chosen environmental indicators in comparison to the base case.

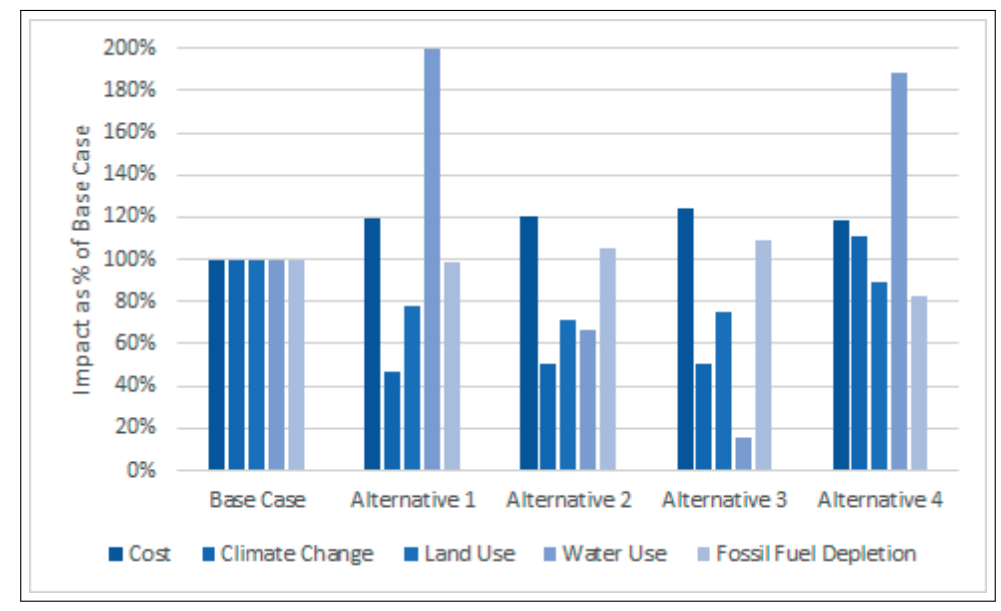

Figure 3.4: Cost and environmental impact of dietary alternatives

Because of the objective function used, the value for climate change is smallest for the Alternative 1 while the biggest improvement in terms of water use is obtained for the Alternative 3 and so on. From the results, it can be seen that for all four alternatives costs are higher than in the base case, while the impact on land use is lower for all 
the environmental impact alternatives. Out of the investigated environmental impact indicators fossil fuel depletion has the smallest improvement potential ( $83 \%$ of the base case) and shows only slight variations between the different alternatives. Water use, in contrast, exhibits considerable fluctuations between alternatives. It is reduced for Alternative 2 and Alternative 3, for the latter lowering its impact to $16 \%$ of the base case but almost doubles for Alternative 1 and 4 . Increases in the other environmental impact factors, while minimising one objective, indicate that trade-offs exist between the different environmental indicators. It is thus often not possible to improve the impact of one indicator without worsening the impact of at least one other. This is an important finding as some of these indicators predominantly affect the local environment while others have implications for the environment on a more global scale. The choice of environmental objective and thus the corresponding alternative depends on the preferences of the decision maker. To decide which one of the alternatives would be preferred to the others, it is thus necessary to have more information on the relative importance of the different environmental impact factors.

\subsubsection{Nutrient intakes}

In order to compare the healthiness and nutritional adequacy of the dietary alternatives, Table 3.5 presents a comparison of nutrient intakes between the Base Case and the four environmental alternatives with reference to the nutritional requirements in the Netherlands. The comparison shows that most of the vitamin and mineral intakes of the dietary alternatives are slightly higher than their intake levels in the base case with the exception of vitamin $\mathrm{C}$ and D. However, the intake levels of the base case only correspond to a small part of the daily recommended levels for these micronutrients (3\% of the daily RDA for vitamin $\mathrm{C}$ and only $1 \%$ for vitamin $\mathrm{D}$ ) while most of the intake for these nutrients comes from other sources that haven't been included in this study, such as for example certain fruits and vegetables in the context of vitamin C. The intake levels of the key nutrients remain at the same level as in the base case with the exception of protein and calcium for which slightly higher levels are recorded for the alternatives. The results further show an increase in the intake of dietary fiber as the consumption of plant-based products increases, while the amount of saturated fatty acids decreases within the dietary alternatives. While slight variations in mineral levels are observed, these changes are negligible for all practical purposes whereas the slight increase in sodium can be explained by the additional consumption of bread within the alternatives. 
Table 3.5: Nutrient intake comparison with reference to daily recommendations

\begin{tabular}{|c|c|c|c|c|c|c|}
\hline & Recommendation $^{1}$ & Base Case & Climate Change & Land Use & Water Use & Fossil Fuel Depletion \\
\hline \multicolumn{7}{|l|}{ Macronutrients: } \\
\hline Protein* & $59 g$ & $26 \%$ & $27 \%$ & $28 \%$ & $28 \%$ & $28 \%$ \\
\hline Dietary Fibre & $25 g^{2}$ & $0 \%$ & $1 \%$ & $5 \%$ & $5 \%$ & $5 \%$ \\
\hline Saturated Fats & $25.6 \mathrm{~g}^{3}$ & $9 \%$ & $9 \%$ & $3 \%$ & $3 \%$ & $3 \%$ \\
\hline \multicolumn{7}{|l|}{ Micronutrients: } \\
\hline Vitamin B12* & $2.8 \mathrm{ug}$ & $64 \%$ & $64 \%$ & $64 \%$ & $64 \%$ & $64 \%$ \\
\hline Vitamin A & 900ug & $3 \%$ & $7 \%$ & $5 \%$ & $5 \%$ & $5 \%$ \\
\hline Vitamin B1 & $1.1 \mathrm{mg}$ & $14 \%$ & $16 \%$ & $17 \%$ & $17 \%$ & $17 \%$ \\
\hline Vitamin B2 & $1.5 \mathrm{mg}$ & $44 \%$ & $47 \%$ & $49 \%$ & $49 \%$ & $49 \%$ \\
\hline Vitamin C & $75 \mathrm{mg}^{2}$ & $3 \%$ & $2 \%$ & $1 \%$ & $1 \%$ & $1 \%$ \\
\hline Vitamin D & $10 \mathrm{ug}^{2}$ & $1 \%$ & $1 \%$ & $0 \%$ & $0 \%$ & $0 \%$ \\
\hline Vitamin E & $10 \mathrm{mg}$ & $1 \%$ & $2 \%$ & $2 \%$ & $2 \%$ & $2 \%$ \\
\hline Folate & $300 u g$ & $8 \%$ & $13 \%$ & $15 \%$ & $15 \%$ & $15 \%$ \\
\hline \multicolumn{7}{|l|}{ Minerals: } \\
\hline Calcium* & $1000 \mathrm{mg}^{2}$ & $44 \%$ & $47 \%$ & $51 \%$ & $51 \%$ & $51 \%$ \\
\hline Zinc* $^{*}$ & $9 \mathrm{mg}$ & $25 \%$ & $25 \%$ & $25 \%$ & $25 \%$ & $25 \%$ \\
\hline Iron* & $9 \mathrm{mg}$ & $3 \%$ & $3 \%$ & $3 \%$ & $3 \%$ & $3 \%$ \\
\hline Potassium & $3500 \mathrm{mg}$ & $18 \%$ & $20 \%$ & $22 \%$ & $22 \%$ & $22 \%$ \\
\hline Magnesium & $350 \mathrm{mg}$ & $13 \%$ & $15 \%$ & $17 \%$ & $17 \%$ & $17 \%$ \\
\hline Sodium & $2.4 \mathrm{~g}^{3}$ & $9 \%$ & $8 \%$ & $10 \%$ & $10 \%$ & $10 \%$ \\
\hline
\end{tabular}

\subsection{Conclusion and Future Research}

The results of this research show that it is possible to construct dietary alternatives to the current consumption of cows' meat and dairy products in the Netherlands that have a lower environmental impact. This is in line with the findings of other research, identifying the replacement of meat and dairy as one of the ways to lower the environmental burden related to our diet (MacDiarmid et al., 2012; Temme et al., 2013; Wilson et al., 2013; Tyszler et al., 2014; Hallström et al., 2015; Van Mierlo et al., 2017). The study further shows, that this partial dietary replacement can be made without a loss of the key nutrients present in cows' meat and dairy products, namely protein, iron, zinc, calcium and vitamin B12. Analysing the impact of the suggested partial dietary changes on the intake of other nutrients in our diet, the study denotes predominantly positive effects from a health perspective. The alternatives provide generally higher amounts of dietary fibre as well as important vitamins and minerals, while at the same time lowering the intake of saturated fatty acids. Thus, it can be argued, that while a full dietary assessment is commonly considered to be the best unit to evaluate nutritional adequacy (Van Kernebeek et al., 2016), healthy alternatives in accordance with current dietary recommendations, replacing only a part of the diet, can be obtained. Moreover, the findings of this study highlight the importance of incorporating the supply chain configuration in a common 
framework with the dietary consumption, when evaluating the environmental impact of these consumption choices. The main reasons for this are, that products are interrelated and that the environmental impact of a product does not only depend on the product itself but also on other factors related to the supply chain of the product, such as where it is produced and how it is processed and transported to its final destination. Furthermore, while most studies in the literature focus on climate change mitigation, i.e. greenhouse gas emissions, this research investigated also other factors affecting the environment, namely land use, water use and fossil fuel depletion. This investigation showed that the optimal solutions in terms of consumption and production of food differed for different environmental indicators. Moreover, trade-offs between these indicators exist, so that it is not necessarily possible to reduce the impact for one of these indicators without increasing another. For future research it could therefore be interesting to incorporate preferences or take a multi-objective approach to obtain more balanced results for the environmental footprint of an alternative. In this context, other elements of sustainability, including social and ethical aspects, should also be included. Furthermore, as studies on meat replacers are becoming more prevalent in the scientific literature (Van Mierlo et al., 2017; Krintiras et al., 2016), future research could include further processing of products to develop a meat replacer instead of the partial dietary consumption considered in this research. Moreover, in order to provide a complete picture of the full environmental impact related to food consumption, future research needs to focus on incorporating the impact of the last stages, e.g. the household phase, in the framework of dietary choices. 



\section{Chapter 4}

\section{Operational Aspects}

A two-echelon inventory-routing problem for perishable products

This chapter is published as:

Rohmer, S. U. K., Claassen, G. D. H., \& G. Laporte (2019). A two-echelon inventoryrouting problem for perishable products. Computers \& Operations Research, 107, 156172 . 


\section{Abstract}

This chapter presents a two-echelon inventory-routing problem for perishable products. Products are delivered from a supplier to an intermediary depot, where storage may occur and from which they are delivered by smaller vehicles to the customer locations. Holding costs are incurred for storage at the depot. Customer availability is taken into account in the form of customer delivery patterns. The objective is to minimise the total transportation and holding costs. We formulate the problem as a mixed integer linear program and solve it by means of an adaptive large neighbourhood search metaheuristic in combination with the solution of a reduced formulation. Three variants of the heuristic are compared on a variety of randomly generated instances. Given the two-stage structure of the problem, computational results show the importance of taking the cost structure into account when choosing the most suitable solution approach. 


\subsection{Introduction}

Last-mile logistics and inventory considerations can play a crucial part in supply chain operations. In fact, the last mile is often considered to be one of the most costly and least efficient stages of the whole supply chain (Gevaers et al., 2009). Transporting products to the final customer can be challenging, especially if these are perishable items, with a limited life span, for which the quality degrades over time. Storage time and time spent on the road affect the quality of the products and reduce their life time at the customer location, and may therefore reduce the value of the product or result in product loss. While perishable products can be found in many areas, the food sector presents an important example of an environment in which quality and safety aspects play an important role (Akkerman et al., 2010), and where high perishability leads to considerable losses and wastage (Yu and Nagurney, 2013). In today's competitive markets, the quality and freshness of a product are important aspects influencing the customers' decision to purchase and hence are vital for the survival of a business.

Last-mile distribution often arises in two-echelon systems that require intermediary storage of products, and needs to consider customer availability during a given time horizon, thus complicating the delivery process. Efficient distribution systems and delivery planning for perishable products can therefore help to avoid spoilage, save costs and positively affect the quality of a product. Periodic vehicle routing as well as inventory management at the depot play an important role in this context, by optimising the delivery schedule, the routes, the storage time and the quantity of products at the depot.

\subsubsection{Literature review}

Problems concerned with the optimal routing of vehicles, to improve delivery operations, have been extensively studied for decades (Cordeau et al., 2007; Laporte, 2009). Over the course of time, several variants of the basic vehicle routing problem (VRP) have incorporated other aspects and more specific requirements related to decision making in the supply chain context (Schmid et al., 2013). A number of studies can be found on the issues related to the routing of perishable products. Thus, Tarantilis and Kiranoudis (2001) developed a metaheuristic for the vehicle routing related to the distribution of fresh milk with a fleet of heterogenous vehicles. In the context of fresh vegetable distribution, Osvald and Stirn (2008) included perishability into the vehicle routing problem with time windows and time-dependent travel times. Amorim and Almada-Lobo (2014) developed a multiobjective model for the vehicle routing problem with time windows to investigate different distribution scenarios and the trade-off between cost and product quality. The problem was solved by using the $\epsilon$-method for small instances and by applying an evolutionary al- 


\section{Operational Aspects}

gorithms for larger instances. Rabbani et al. (2015) proposed a multi-objective VRP with time windows and customer selection, assuming a heterogenous fleet of vehicles and considering multiple deteriorating products. Wang et al. (2016) solved a multi-objective VRP with time windows and perishability considerations using a two-phase heuristic method based on a variable neighbourhood search and a genetic algorithm. Rabbani et al. (2016) considered the use of multiple middle depots and incorporated several aspects, such as product freshness and profit maximisation into the objective function. They developed a genetic algorithm for the solution of large instances. Considering perishability in a site-dependent vehicle routing problem with time windows and a heterogeneous fleet of vehicles, Amorim et al. (2014) developed a neighbourhood search algorithm and applied it to a real-life case study arising in a Portuguese food distribution company. Hsu et al. (2007) extended the vehicle routing problem with time windows by adding a stochastic cost component related to the perishability of products. Song and Ko (2016) proposed a non-linear model with the objective of maximising customer satisfaction related to the delivery of multi-commodity perishable products with refrigerated and non-refrigerated vehicles. The problem was solved using a priority-based heuristic approach.

A number of extensions exist on the integration of other aspects of the planning process into routing models, such as production, location and inventory decisions. While the focus in the following will be on the latter aspect, examples related to other features can be found in Farahani et al. (2012), Govindan et al. (2014) and the review of Amorim et al. (2013).

Nahmias (2011) and Karaesmen et al. (2011) provide reviews related to the management and modelling of perishable inventory systems. For a more general and extensive overview of the field of inventory-routing problems (IRP), its variants and associated solution approaches we refer to the reviews of Bertazzi et al. (2008), Andersson et al. (2010) and Coelho et al. (2013).

In the context of perishable products, Hiassat and Diabat (2011) proposed an integrated model for a location-inventory-routing problem considering products with a limited lifespan. Le et al. (2013) developed an algorithm for an IRP based on column generation and cutting planes, the problem is extended in Hiassat et al. (2017), integrating location decisions into the model, and solved using a genetic algorithm. Coelho and Laporte (2014) applied branch-and-cut to optimally solve the perishable inventory-routing problem (PIRP) under general assumptions and consideration of two different selling policies. Jia et al. (2014) solved an IRP for perishable products with multiple time windows and loading costs, solving the problem using a two-phase solution algorithm. Mirzaei and Seifi (2015) considered the impact of lost sales in their inventory-routing problem. The resulting mixed integer non-linear programming model was solved using a metaheuristic based on simulated annealing and tabu search. Kande et al. (2015) proposed a tabu search metaheuristic for a routing problem with inventory and lot-sizing decisions as well as mul- 
tiple source nodes. Dealing with uncertain demand in a multi-period IRP model, Soysal et al. (2015) further included environmental aspects in the form of greenhouse gas emissions and fuel consumption. Rahimi et al. (2017) developed a multi-objective model for the IRP of perishable products, allowing for a choice of different vehicles. They incorporated environmental aspects as well as customer satisfaction considerations on top of the traditional cost minimisation. The problem was solved by means of a genetic algorithm. Diabat et al. (2016) proposed a new arc-based formulation and a tabu search algorithm for the inventory-routing problem for perishable products. Azadeh et al. (2017) considered an inventory-routing problem with transshipments for a perishable product and applied a genetic algorithm to solve the problem. Li et al. (2016) developed a mixed integer linear programming model for perishable supply chains, incorporating production decisions in the inventory-routing problem and maximising profit. In addition, Zhao et al. (2008) proposed a similar structured two-echelon inventory routing problem without perishability considerations. The problem was solved using a variable large neighbourhood search. Table 4.1 provides a summary of the related scientific literature. 


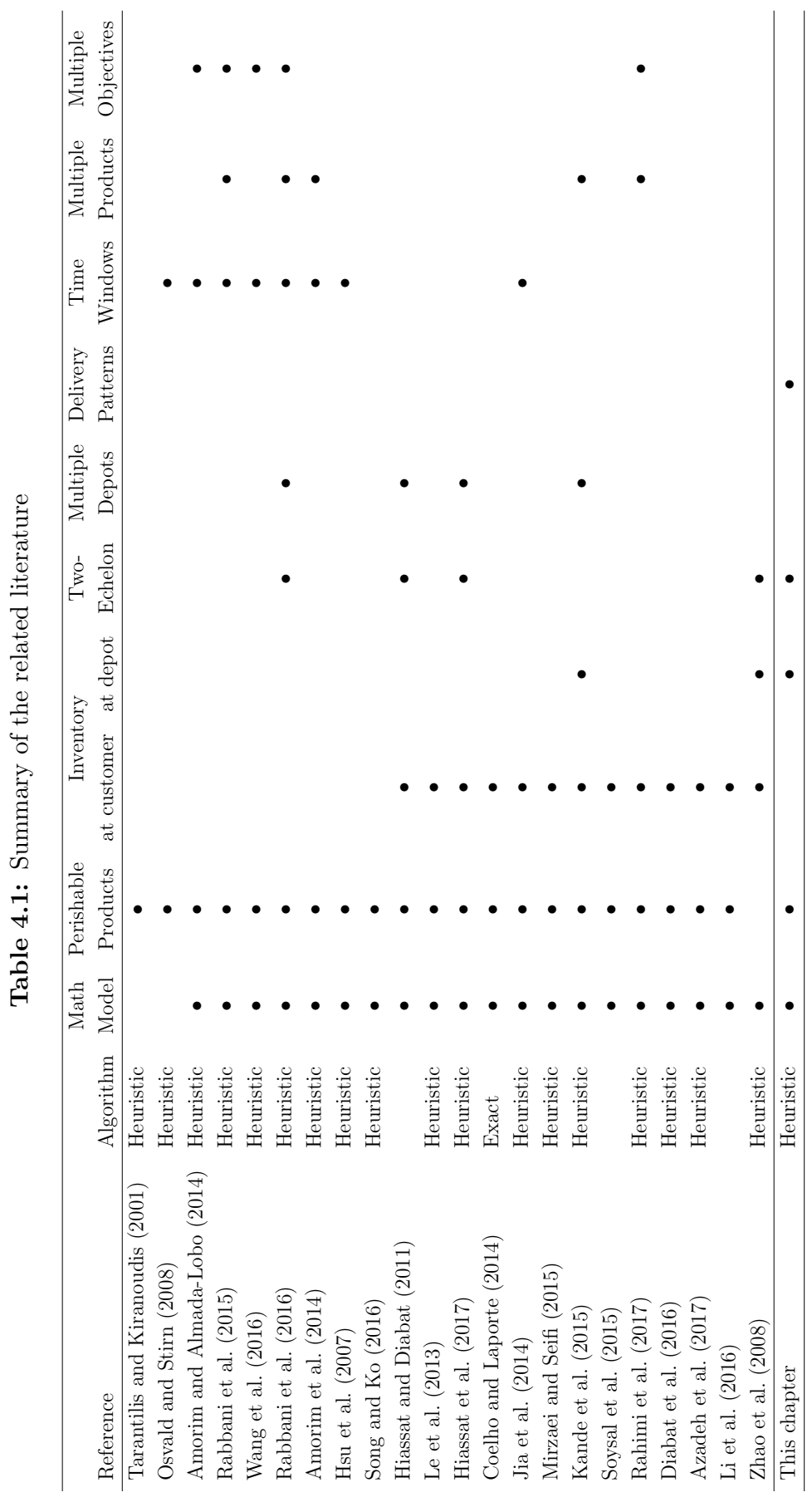




\subsubsection{Contribution and organisation of this chapter}

This chapter focuses on inventory-routing for perishable products in the context of lastmile city distribution. Given this focus, it is reasonable to assume a multi-level system, resulting in a two-echelon routing problem (Hemmelmayr et al., 2012). A survey of twoechelon routing problems can be found in Cuda et al. (2015). This study, like many others, is about the solution of a last-mile distribution system in a two-echelon setting. However, our problem differs from most existing two-echelon problems in two main ways. First, we consider inventory at the depot and not at the customer locations as is the case in many papers. Second, we are the first to handle multiple delivery patterns in the context of a two-echelon system. Our aim is to introduce the two-echelon perishable inventory-routing problem, model it and solve it heuristically through an adaptive large neighbourhood search (ALNS).

The chapter is organised as follows. In Section 4.2 a formal description of the problem will be given. Section 4.3 introduces the mathematical formulation of the model, while Section 4.4 describes the heuristic. Computational results follow in Section 4.5, and conclusions are presented in Section 4.6.

\subsection{Formal Problem Description}

We consider the inventory-routing problem for perishable products within the context of urban last-mile delivery. We therefore assume a two-echelon system, with a supplier, an intermediary depot and several customer locations. Fresh products are delivered from the supplier to the depot and then stored until delivery occurs. Inventory levels are updated at the beginning of each day, representing a time period. The depot, which belongs to the supplier, receives flower or vegetable deliveries from producers. The customers, on the other hand, are independent and need to be served according to their availability and preferences. The availability of a customer is provided in the form of combinations of visit periods. These delivery day combinations are represented for each location as a list of combinations of daily time windows, during which deliveries can be made, as it is the case in the periodic vehicle routing problem (PVRP) presented by Cordeau et al. (1997). An example of this would be a customer needing to be supplied with fresh produce every two days and delivery could take place either on days $1,3,5$ and 7 or on days 2,4 and 6. The rationale is that customers will receive a new order of products every couple of days to guarantee freshness of the product and fulfill the customer demand. This means that the departures of the vehicles need to be scheduled according to the delivery time windows at the customer location. This is because customers will commonly not accept 


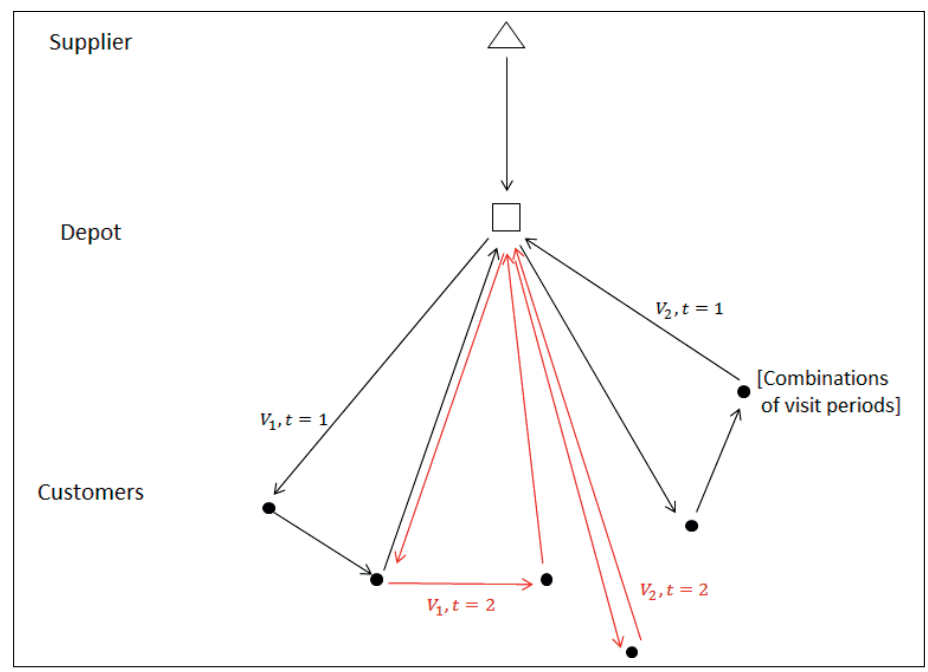

Figure 4.1: Two-echelon delivery system with periodic routing

delivery every day but rather follow a certain delivery pattern. An overview of the system is depicted in Figure 4.1.

The problem is defined over a limited time horizon of a week and the solution must satisfy the periodic customer demands. Customer demands are assumed to be deterministic and known for each period at the beginning of the weekly time horizon. The supplier has enough capacity to satisfy the demand and can deliver to the depot within a reasonably short-time frame. It is therefore possible to deliver goods to the depot and thence to different customer locations within the same day, i.e. a time period. Vehicle capacity, however, is limited and each delivery to the depot incurs a linehaul travel cost.

Products can be stored at the depot up to a certain capacity or until they are discarded as waste due to their perishable nature. Perishable products can be generally categorised into two types. The first type is associated with an expiry date, meaning that the products are suitable for consumption up until a certain point in time, after which they are discarded (Nahmias, 2011). This is often the case for dairy products for example. The second type relates to a gradual decrease in product quality and can be for example observed for salads, fruits and bread (Rong et al., 2011). The focus in this research will be on the latter type. Thus, the deterioration of a product occurs over time in relation to the age of the product. The cost of this deterioration is included in the inventory holding cost. From the depot to the customer, delivery is carried out by a homogeneous fleet of vehicles. Vehicles are readily available at the depot, though limited with respect to their capacity. 
The problem consists of the following decisions:

- When and how much to deliver from the supplier to the depot?

- When and how much to deliver from the depot to the customers?

- What is the optimal routing from the depot to the customer locations for the different time periods?

The aim is therefore to determine an optimal delivery schedule and routing to the customer locations during each time period, and optimise the storage time of the product at the depot, minimising both the routing and inventory costs, while accounting for the loss of freshness of the product over time.

\subsection{Mathematical Formulation}

This section introduces the notations and parameters used and provides a formal description of the mixed integer linear programming (MILP) formulation based on the assumptions presented in Section 4.2.

Table 4.2: Summary of notation

\begin{tabular}{cl}
\hline Sets & set of nodes indexed by $i, j, l\{$ depot: $0 ;$ customer: $1, \ldots, n\}$ \\
$N$ & set of arcs $(i, j): i, j \in N, i \neq j$ \\
$A$ & set periods indexed by $t$ \\
$T$ & set of vehicles indexed by $k: k \in\{1, \ldots, m\}$ \\
$K$ & set of product ages indexed by $g$ \\
$G$ & set of visit combinations of $i$ \\
$R_{i}$ & \\
Parameters & routing costs on arc $(i, j): i, j \in\{0, \ldots, n\}$ \\
$c_{i j}$ & linehaul routing cost supplier-depot-supplier \\
$C$ & demand of customer $i$ in period $t$ \\
$d_{i}^{t}$ & capacity of vehicle $k(k=0:$ supplier-depot; $k=1,2,3:$ depot-customer) \\
$Q^{k}$ & inventory holding capacity at depot \\
$H$ & unit inventory holding cost at depot (including deterioration cost) for product age $g$ \\
$h^{g}$ & 1 if day $t$ belongs to visit combination $r$ \\
$a^{r t}$ & \\
Variables & q if customer $j$ is visited immediately after customer $i$ by vehicle $k$ in period $t$ \\
$x_{i j}^{k t}$ & 1 if vehicle $k$ visits customer $i$ in period $t$ \\
$y_{i}^{k t}$ & 1 if visit combination $r$ of customer $i$ chosen \\
$z_{i}^{r}$ & number of vehicles supplier-depot in period $t$ \\
$u^{t}$ & quantity delivered of age $g$ from depot to customer $i$ in period $t$ by vehicle $k$ \\
$v_{i}^{g k t}$ & quantity delivered from supplier to depot in period $t$ \\
$w^{t}$ & position of customer $i$ in the routing of vehicle $k$ in time period $t$ \\
$I^{g t}$ & $s_{i}^{k t}$
\end{tabular}




\section{Operational Aspects}

The problem is then:

Minimise $\sum_{t \in T} C u^{t}+\sum_{g \in G} \sum_{t \in T} h^{g} I^{g t}+\sum_{i \in N} \sum_{j \in N} \sum_{k \in K} \sum_{t \in T} c_{i j} x_{i j}^{k t}$

subject to

$$
\begin{aligned}
& I^{g t}=I^{g-1, t-1}-\sum_{i \in N} \sum_{k \in K} v_{i}^{g-1, k, t-1} \quad g \in G \backslash\{0\}, t \in T \backslash\{0\} \\
& I^{0 t}=w^{t} \quad t \in T \\
& I^{g t} \geq \sum_{i \in N \backslash\{0\}} \sum_{k \in K} v_{i}^{g k t} \quad g \in G, t \in T \\
& \sum_{g \in G} I^{g 0}=w^{0} \\
& \sum_{g \in G} I^{g t} \leq H \quad t \in T \\
& \sum_{r \in R_{i}} a^{r t} d_{i}^{t} z_{i}^{r}=\sum_{g \in G} \sum_{k \in K} v_{i}^{g k t} \quad i \in N \backslash\{0\}, t \in T \\
& w^{t} \leq H-\sum_{g \in G} I^{g, t-1} \quad t \in T \\
& \sum_{g \in G} \sum_{i \in N \backslash\{0\}} v_{i}^{g k t} \leq Q^{k} y_{0}^{k t} \quad k \in K, t \in T \\
& w^{t} \leq Q^{0} u^{t} \quad t \in T \\
& \sum_{k \in K} y_{i}^{k t} \leq 1 \quad i \in N \backslash\{0\}, t \in T \\
& y_{i}^{k t} \leq \sum_{j \in N} x_{i j}^{k t} \quad i \in N, k \in K, t \in T \\
& \sum_{r \in R_{i}} z_{i}^{r}=1 \quad i \in N \backslash\{0\} \\
& \sum_{i \in N} \sum_{k \in K} x_{i j}^{k t}-\sum_{r \in R_{j}} a^{r t} z_{j}^{r}=0 \quad j \in N \backslash\{0\}, t \in T \\
& \sum_{i \in N} x_{i j}^{k t}-\sum_{l \in N} x_{j l}^{k t}=0 \quad k \in K, t \in T, j \in N \\
& s_{i}^{k t}-s_{j}^{k t}+n x_{i j}^{k t} \leq n-1 \quad i, j \in N \backslash\{0\}, t \in T, k \in K \\
& v_{i}^{g k t}, w^{t}, I^{g t}, s_{i}^{k t} \geq 0 \\
& x_{i j}^{k t}, y_{k t}, z_{i}^{r} \in\{0,1\} \\
& u^{t} \in \mathbb{Z} \text {. }
\end{aligned}
$$


The objective function (4.1) minimises the sum of the delivery cost, consisting of linehaul travel and customer routing cost, and of the inventory cost. Constraints (4.2) and (4.3) are inventory constraints related to the age of the product. Constraints (4.4) requires a delivery to update the inventory in period zero. Constraints (4.5) and (4.6) ensure that inventory levels cover at least the delivery during the same period while also not exceeding the inventory capacity at the depot. Constraints (4.7) mean that the demand at each consumer is met for the chosen delivery pattern. Constraints (4.8) restrict the amount that can be delivered to the depot depending on depot capacity and existing inventory. Constraints (4.9) and (4.10) impose a vehicle capacity for delivery to the customer and the delivery to the depot. Constraints (4.11) state that delivery to a customer is made by only one vehicle, while constraints (4.12) mean that a delivery can only be made by an activated vehicle. Constraints (4.13) assign a delivery pattern to each customer and constraints (4.14) ensure that delivery can only occur on days belonging to the chosen delivery pattern. Constraints (4.15) state that each vehicle that visits a customer also leaves the customer. Constraints (4.16) are standard subtour elimination constraints. Constraints (4.17) to (4.19) enforce the non-negativity and integrality of the variables.

\subsection{Heuristic}

For very small instances, the problem can be solved to optimality by a standard integer linear programming solver, whereas this is not feasible for larger-size instances. We therefore propose a two-stage matheuristic, i.e. a "heuristic algorithm[ ] made by the interoperation of metaheuristics and mathematical programming techniques" (Boschetti et al., 2009), combining an adaptive large neighbourhood search (ALNS) with the solution of a MILP formulation, in order to solve the problem for more realistic instances. This two-stage approach allows the exploitation of the two-echelon structure of the problem by splitting it into routing and linehaul related decisions. The overall performance of the heuristic, however, depends on the cost structure of the instances, thus determining the order in which the different components of the heuristic are solved. As a result, three variants obtained by altering the structure of the heuristic, are proposed in this research.

\subsubsection{Heuristic Variant 1}

In the first variant, the MILP model is solved first, determining optimal customer delivery patterns, linehaul travel and the inventory of products at the depot. Based on this optimal solution, the ALNS then aims to find good solutions for the second-stage routing problem, 
delivering each customer according to the optimal delivery patterns determined in the first stage. The structure of the approach is described in pseudo-code in Algorithm 1.

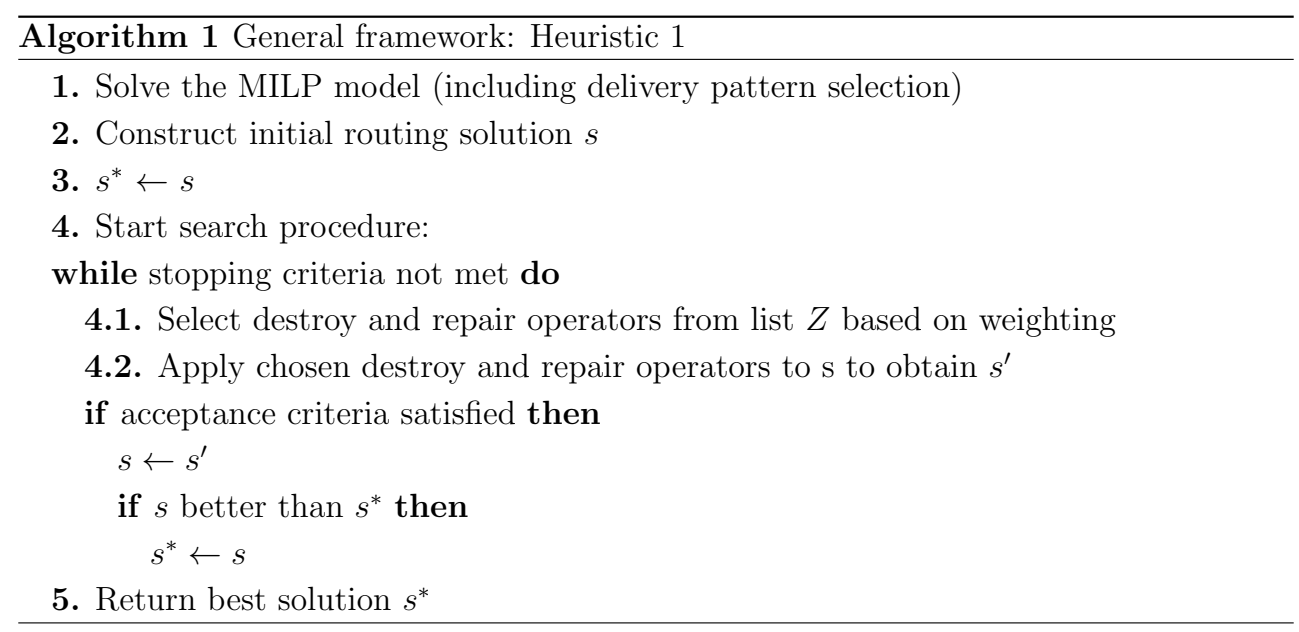

Solution of a MILP and construction of an initial routing solution

The MILP formulation used to solve the first stage of the problem is a reduced version of the mathematical formulation provided in Section 4.3. Note that while most parameters and variables remain the same, the variables $v_{i}^{g k t}$ are replaced with the variables $v^{g t}$, and new variables $d^{t}$ are added to the model in order to determine the aggregated demand for each period $t$. The detailed mathematical formulation is provided in the following:

$$
\text { Minimise } \quad \sum_{t \in T} C u^{t}+\sum_{g \in G} \sum_{t \in T} h^{g} I^{g t}
$$

\section{subject to}

$$
\begin{aligned}
& d^{t}=\sum_{g \in G} v^{g t} \quad t \in T \\
& I^{g t}=I^{g-1, t-1}-v^{g-1, t-1} \quad g \in G \backslash\{0\}, t \in T \backslash\{0\} \\
& I^{0 t}=w^{t} \quad t \in T \\
& I^{g t} \geq v^{g t} \quad g \in G, t \in T \\
& \sum_{g \in G} I^{g 0}=w^{0}
\end{aligned}
$$




$$
\begin{aligned}
& \sum_{g \in G} I^{g t} \leq H \quad t \in T \\
& \sum_{i \in N \backslash\{0\}} \sum_{r \in R_{i}} a^{r t} d_{i}^{t} z_{i}^{r}=\sum_{g \in G} v^{g t} \quad t \in T \\
& w^{t} \leq H-\sum_{g \in G} I^{g, t-1} \quad t \in T \\
& w^{t} \leq Q^{0} u^{t} \quad t \in T \\
& \sum_{r \in R_{i}} z_{i}^{r}=1 \quad i \in N \backslash\{0\} \\
& v_{g t}, w^{t}, I^{g t} \geq 0, \quad z_{i}^{r} \in\{0,1\}, \quad u^{t} \in \mathbb{Z} .
\end{aligned}
$$

Once the MILP model is solved, an insertion heuristic (see Algorithm 2) is applied in order to determine the routing for each period $t$ based on the previously selected customer delivery patterns. For each day, the customers allocated to the corresponding daily delivery list are chosen randomly and inserted in the best feasible position under consideration of all the daily routes. If no feasible insertion can be found due to the capacity restrictions, a new route is created and the customer is inserted in it.

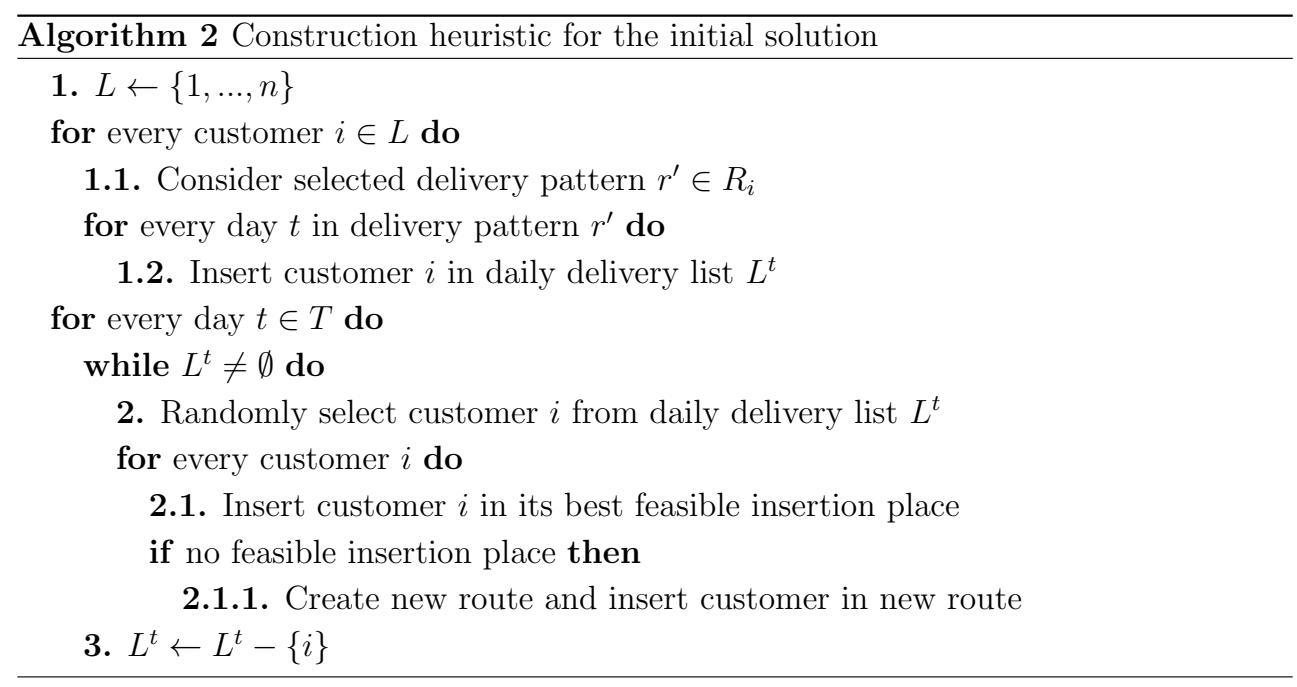

\section{Destroy operators}

We have developed a number of different destroy operators, operating at the customer and route level. The operators remove a percentage $\mathrm{Nb}$ of the customers from the current solution. 
Random Customer Removal: This is a standard operator in which customers are selected randomly and removed from their current route.

Worst Customer Removal: For a random day, this operator identifies the worst customer according to its insertion cost in the current solution. This customer with the largest insertion cost or savings potential is then removed from the solution.

Related Customer Removal: This operator is based on the related customer removal operators used by Shaw (1998) and Azi et al. (2014). However, while Azi et al. (2014) build on Shaw (1998) by defining a proximity measure based on spatial and temporal distance, we apply two variants of the operator. The first uses a spatial distance measure, so that $z_{i l}=c_{i l}$, while the second applies a distance measure based on the difference in demands between customers, so that $z_{i l}=\left|d_{i}^{t}-d_{l}^{t}\right|$. The structure of the operator is described in Algorithm 3.

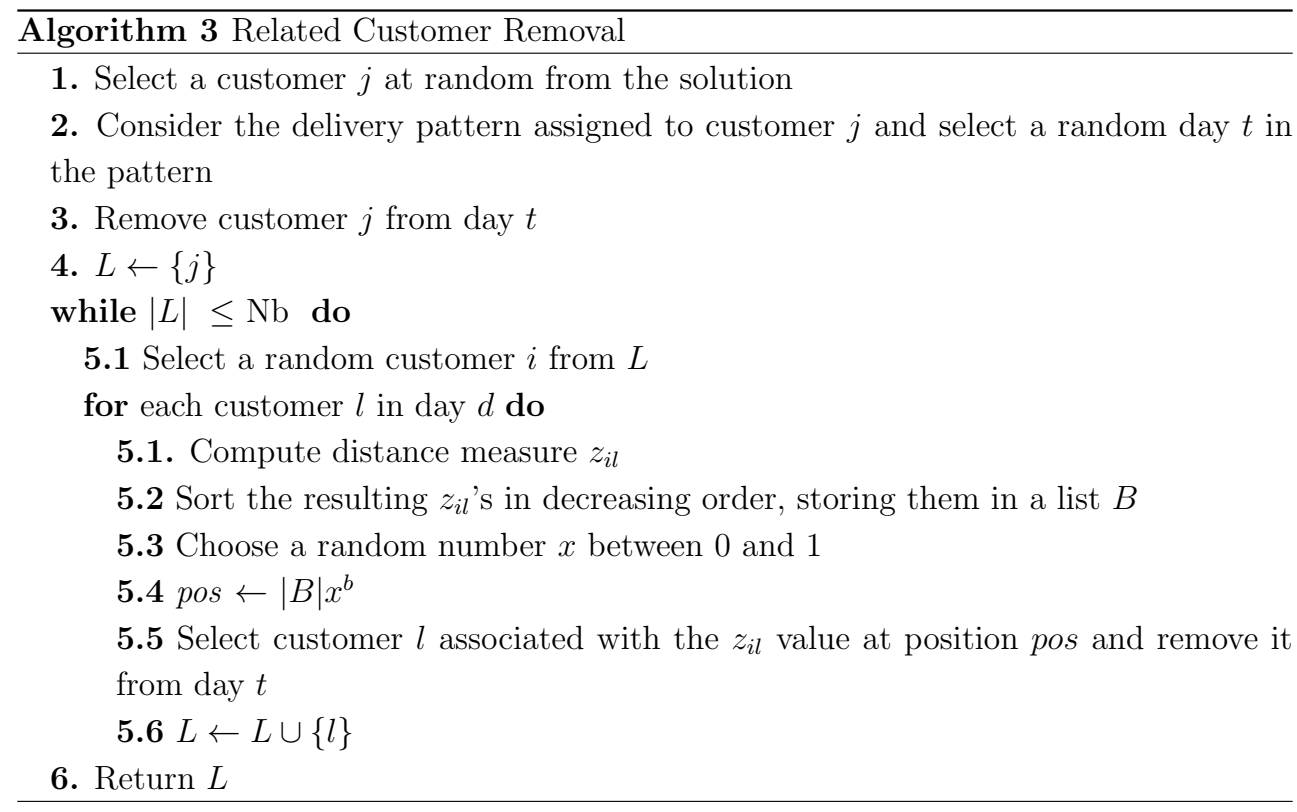

Parameter $b$ in 5.4 regulates the intensity of the bias towards closer customers. As a result of the tuning of this parameter, $b$ was set to 10 in our implementation.

Random Route Removal: For a random day, the operator selects a number of routes at random and removes them from the solution. 


\section{Repair operator}

Customers that have been removed during the destruction procedure need to be reinserted using a repair operator. The operator used for this is based on cheapest insertion and follows the same insertion procedure as in the construction heuristic. Thus, for each day, customers are selected from the list of removed customers and reinserted into the solution in the cheapest feasible position. This process is repeated until, for each day, all of the daily customers are again part of the solution.

\section{Acceptance criterion and adaptive mechanism}

The acceptance criteria for candidates is based on a simulated annealing rule, as in Ropke and Pisinger (2006). The adaptive mechanism is only applied to the destroy operators in this setting, since the options to repair a solution are limited to the cheapest insertion operator. The heuristic terminates after a fixed number of iterations.

\subsubsection{Heuristic Variant 2}

The second variant integrates the selection of customer delivery patterns within the routing decision, constructing daily delivery routes before optimally solving the linehaul and inventory part of the problem. The MILP model is thus integrated into the ALNS framework and solved for each of the found solutions with alternative delivery patterns. The structure of the approach is described in pseudo-code in Algorithm 4.

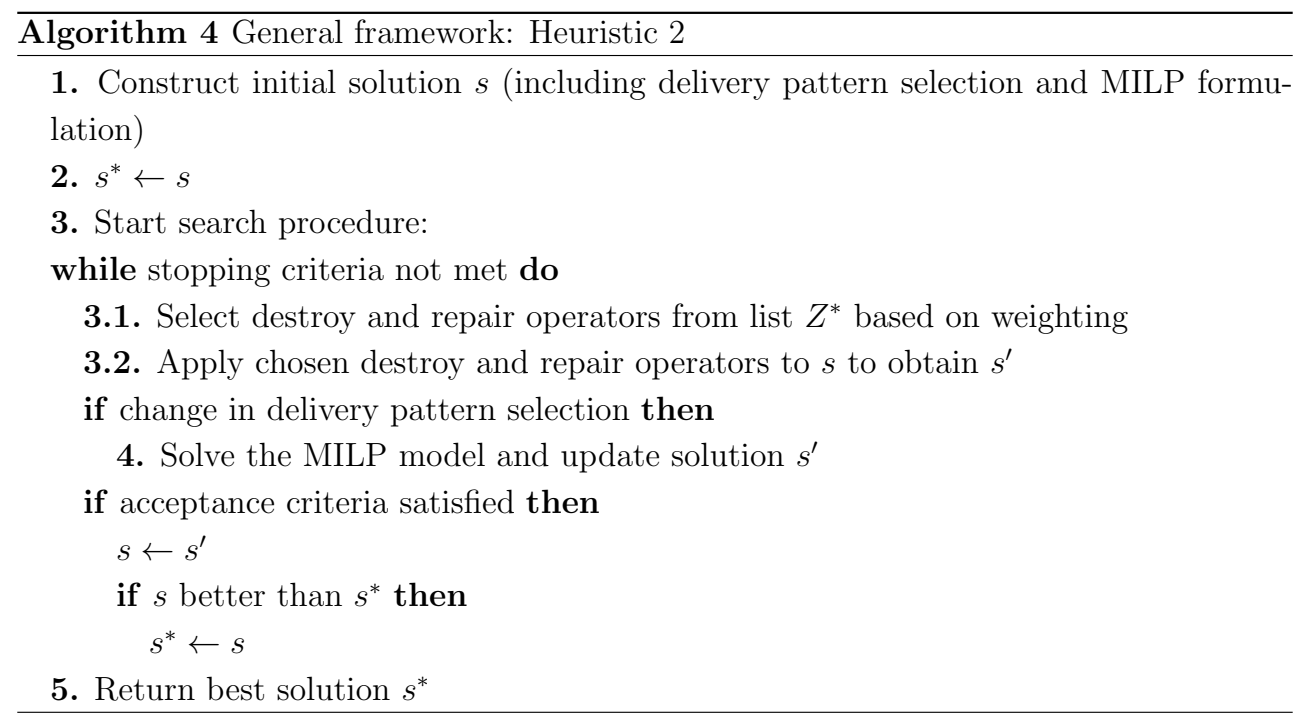




\section{Operational Aspects}

\section{Construction of an initial solution}

Whereas in the previous variant, the pattern selection was predetermined by the MILP model, here the construction heuristic starts by randomly selecting a delivery pattern for each customer. Once every customer has been assigned a delivery pattern, the procedure is identical to the previous construction heuristic, with the exception that a MILP model is solved at the end in order to determine the linehaul and storage component of the problem under consideration of the chosen delivery patterns. An overview of the construction heuristic can be found in Algorithm 5. The corresponding MILP model is a simplified version of the model presented in Section 4.4.1, for which the objective function and most of the constraints remain the same. However, the expressions $d_{t}$, representing the daily aggregated demand, are now predetermined by the heuristic and thus become parameters in the model, while constraints (4.27) and (4.30), linked to the pattern selection, are eliminated from the model, making the notations $z_{i}^{r}$ and $a^{r t}$ irrelevant.

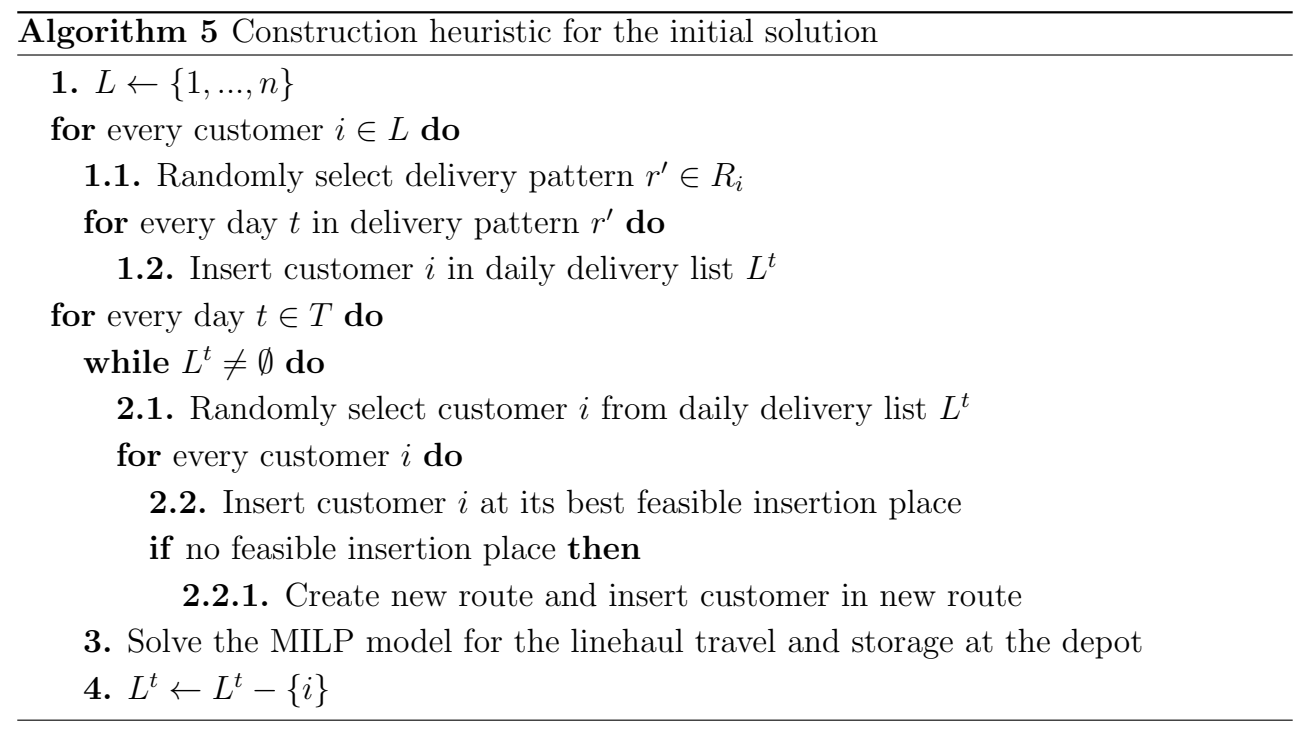

\section{Destroy Operators}

In addition to the destroy operators of the first heuristic variant, the second variant also includes an operator at the delivery pattern level to allow for changes in the selection of customer delivery patterns.

Random Customer and Pattern Removal: The idea is the same as in the standard customer removal, but in addition to removing a random customer from all the routes, 
the operator also removes the record of the customer's delivery pattern from the solution, storing the customer in a list of unassigned customers without a selected delivery pattern.

\section{Repair Operators}

Similar to the destroy operators, the repair operators consist of those used in the previous variant and of a number of new operators related to the selection of customer delivery patterns. Note, that the use of repair operators depends on the preceding destroy operator, i.e. whether the operator affects solely the customer level or both the customer and the delivery pattern level. If both levels are affected, the heuristic first chooses an operator to select the delivery pattern and assign the customer to delivery days before choosing another operator to insert the customer in the routing solution.

Random Pattern Selection: The random pattern selection operates in the same manner as the pattern selection in the construction heuristic, where a delivery pattern is chosen at random from the list of customer specific delivery patterns.

Balanced Pattern Selection: This operator takes a more balanced approach for the pattern selection by applying a customer density measure $\rho$. Note that two different customer density scores are considered in this research. The first is based on the sum of the number of customers in each day of the pattern, while the second is based on the sum of the daily demands of each day in the pattern. The detailed structure of the operator is described in Algorithm 6.

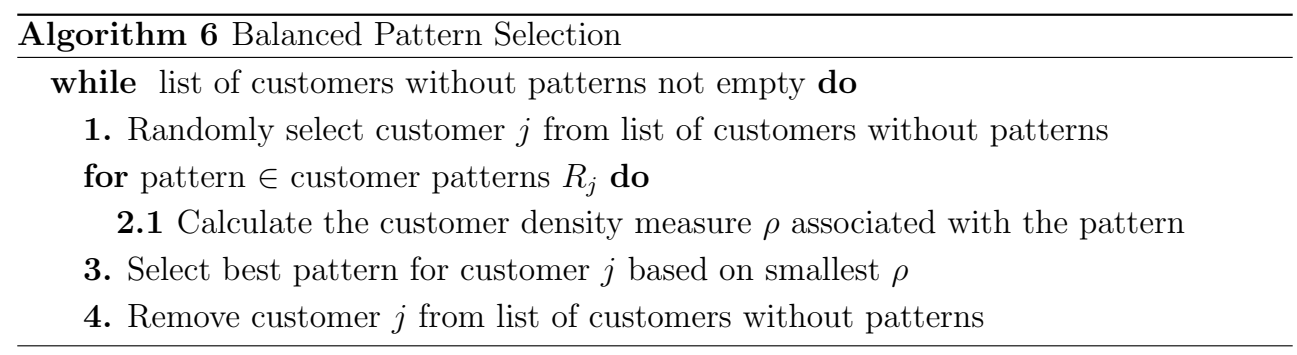

\subsubsection{Heuristic Variant 3}

We have developed a third heuristic variant consisting of a hybrid of the first two variants. In this variant, the initial solution is generated in the same way as for variant 1 (i.e. the MILP model of variant 1 for the linehaul is solved first), while the general structure of the heuristic follows variant 2 . This means that variant 3 starts with the optimal cost from 
the linehaul perspective but allows for more flexibility since the linehaul problem can be solved for different customer patterns using the MILP model of variant 2.

\section{Acceptance criterion and adaptive mechanism}

We use the same acceptance criterion as in the first variant, with the exception that the adaptive mechanism is applied to both the destroy operators and the repair operators used to restore customer patterns.

\subsection{Computational Results}

We have carried out a number of computational experiments in order to validate the MILP formulation and test the heuristic approaches proposed in this study. The experiments were designed to investigate the effect of the cost structure on the performance of the three heuristic variants. We have coded the heuristics in Python3.5 and CPLEX 12.6 running on a single thread. All computations were executed on a machine equipped with an Intel(R) Xeon(R) X5675 processor running at 3.07GHz.

\subsubsection{Instance description}

The heuristics were tested on two sets of instances, each consisting of 90 small-size instances with up to 150 customers. The two sets differ with respect to the size of the grid in which the customers are located. In the first set of instances the customers are located in a $2 \times 2$ square (in $\mathrm{km}$ ), i.e. small grid, while in the second set the customers are located in a $25 \times 25$ square (in $\mathrm{km}$ ), i.e. large grid. In both cases, the depot is located at $(2.5,2.5)$. The distance for the linehaul travel is set at one of the following values for both sets: $20 \mathrm{~km}, 40 \mathrm{~km}$ or $80 \mathrm{~km}$. Thus, the two different grid sizes impact the relations between the cost components of the objective function, as linehaul and inventory costs remain in the same range. This means that in the case of the large grid instances, the routing cost contributes relatively more to the total cost. The calculation of the routing $\operatorname{costs} c_{i j}$ is based on the Euclidean distances between the locations in the plane and include fuel, wage and vehicle costs per $\mathrm{km}$. 
Table 4.3: Routing cost components per vehicle type

\begin{tabular}{lcc}
\hline & Light Duty Vehicle & Medium Duty Vehicle \\
\hline Average speed & $30 \mathrm{~km} / \mathrm{h}$ & $70 \mathrm{~km} / \mathrm{h}$ \\
Fuel consumption & $30 \mathrm{l}$ per $100 \mathrm{~km}$ & $15 \mathrm{l}$ per $100 \mathrm{~km}$ \\
Fuel cost & $\mathbf{0 . 4 2} € / \mathbf{k m}$ & $\mathbf{0 . 2 1} € / \mathbf{k m}$ \\
Driver's wage & $9.5 € / \mathrm{h}$ & $12.5 € / \mathrm{h}$ \\
Wage costs & $\mathbf{0 . 3} € / \mathbf{k m}$ & $\mathbf{0 . 1 8} € / \mathbf{k m}$ \\
Truck payment and insurance & $0.3 € / \mathrm{km}$ & $0.3 € / \mathrm{km}$ \\
Maintenance and repairs & $0.15 € / \mathrm{km}$ & $0.15 € / \mathrm{km}$ \\
Vehicle costs & $\mathbf{0 . 4 5} € / \mathbf{k m}$ & $\mathbf{0 . 4 5} € / \mathbf{k m}$ \\
\hline Total routing cost per $\mathbf{k m}$ & $\mathbf{1 . 1 7} € / \mathbf{k m}$ & $\mathbf{0 . 8 4} € / \mathbf{k m}$ \\
\hline
\end{tabular}

The inventory costs increase exponentially and are calculated based on the formula $h^{g}=$ price $\times p \times f^{t}$, where the price of the product is randomly selected from the interval $[10$, $30], p$ is a percentage in $[0.02,0.04], f$ is a positive growth factor set at 2 , and $t$ is the time period. The planning horizon in this research is set for all instances to $T=5$. Five delivery patterns consisting of different combinations of delivery days $(\{1,3\},\{1,4\},\{2,4\}$, $\{2,5\},\{3,5\})$, are considered and each customer is assigned two delivery patterns chosen at random. Based on the instances of Song and Ko (2016), the customer demands are volume based and range between $0.3 \mathrm{~m}^{3}$ and $1.8 \mathrm{~m}^{3}$, the capacity of the vehicles used for the customer routing is set at $12 \mathrm{~m}^{3}$. The vehicle capacity for the linehaul travel is $38 \mathrm{~m}^{3}$, which corresponds to the standard size of a small shipping container in Europe. For the small grid instance structure the capacity at the depot is $50 \mathrm{~m}^{3}$ for instances with up to 40 customers and $150 \mathrm{~m}^{3}$ for larger instances. The large grid instance structure features a larger inventory capacity at the depot of $100 \mathrm{~m}^{3}$ for instances of up to 40 customers and $200 \mathrm{~m}^{3}$ for larger instances.

\subsubsection{Parameter settings}

For the parameter tuning of the three heuristics, two sets of 18 test instances were selected at random, representing the two different instance structures considered in this research. The tuning for these two test sets was carried out separately. We executed 10 runs for each parameter setting of the heuristics, and the setting with the best average deviation from the best found solution was chosen. The results of the tuning were based on a search consisting of 25,000 iterations and a segment size of 200 iterations, as this resulted in a good trade-off between run time and solution quality. Three different intervals for the percentage of destruction (i.e. the percentage of customers to remove from the solution) were reviewed, namely $[10 \%, 30 \%],[20 \%, 40 \%],[30 \%, 50 \%]$. All other parameter values 
were initially set equal to those of Ropke and Pisinger (2006) and then sequentially altered in the tuning phase. The resulting best parameter setting for each heuristic variant and instance structure is shown in Table 4.4.

Table 4.4: Parameter settings

\begin{tabular}{ccccccc}
\hline & \multicolumn{3}{c}{ Instance structure 1 } & \multicolumn{3}{c}{ Instance structure 2 } \\
Parameters & Variant 1 & Variant 2 & Variant 3 & Variant 1 & Variant 2 & Variant 3 \\
\hline $\begin{array}{c}\text { Percentage of destruction: } \\
\text { Acceptance criterion: }\end{array}$ & $30 \%-50 \%$ & $30 \%-50 \%$ & $30 \%-50 \%$ & $30 \%-50 \%$ & $30 \%-50 \%$ & $30 \%-50 \%$ \\
$w$ & & & & & & \\
$c$ & 0.001 & 0.001 & 0.001 & 0.001 & 0.001 & 0.001 \\
$c$ & 0.99976 & 0.99974 & 0.99983 & 0.99976 & 0.99983 & 0.99985 \\
Weight adjustment: & & & & & & \\
$\sigma_{1}$ & 33 & 22 & 44 & 22 & 44 & 33 \\
$\sigma_{2}$ & 9 & 13.5 & 4.5 & 13.5 & 4.5 & 9 \\
$\sigma_{3}$ & 13 & 19.5 & 6.5 & 19.5 & 6.5 & 13 \\
$r$ & 0.1 & 0.1 & 0.1 & 0.5 & 0.5 & 0.5 \\
\hline
\end{tabular}

\subsubsection{Results}

This section presents the results for the two instance structures and three heuristic variants based on the best parameter settings identified in Section 4.5.2. For very small instances, of up to ten customers, these results are compared with the optimal solution values found by the mathematical model, while for larger instances it is no longer possible to solve the problem to optimality. The run time for solving the model to optimality differs considerably between the two instance structures, as well as between individual instances. For the small grid instance structure, the model obtains an optimal solution for instances with 10 customers on average within 86 seconds, while for the large grid instance structure the run time is considerably longer, with an average of about 5,800 seconds. In addition, one of the large grid instances could not be solved to optimality, with a remaining optimality gap of $6.63 \%$ after running the model for several days. Increasing the number of customers to $n=15$ for the small grid instances leads to a considerable increase in the run time, resulting in an average run time of 7435.5 seconds.

The column headings of Tables 4.5 and 4.7 present the linehaul cost structure, the optimal solution value found by the mathematical model, as well as the performance of the heuristic variants for each of the small and large grid instances, respectively. The total cost term for the optimal solutions is broken down into the different cost components, namely the first echelon cost $\left(1^{s t} E\right)$, consisting of linehaul (LC) and inventory cost (IC), and the routing cost $(\mathrm{RC})$. For the heuristic variants the tables provide the best solution values found, as well as the performance of the algorithms in terms of time and deviation. Best and average deviations are computed with respect to the optimal solution values found 


\section{A Two-Echelon Inventory-Routing Problem for Perishable Products}

for each of the instances. The comparison of the optimal solution values with the three heuristic variants for instances with $n=10$ in Table 4.5 shows that the variants manage on occasions to find optimal or close to optimal solutions for most small grid instances. On average, variant 3 performs best with an optimality gap of $1.79 \%$, followed by variant 1 with a gap of $2.12 \%$, and variant 2 with a gap of $2.48 \%$. In terms of finding optimal or close to optimal solutions, variant 2 performs best, closely followed by variant 3 , and considerably outperforming variant 1 . For instances with 15 customers, the comparison of the optimal solution values with the three heuristic variants shows that each variant still finds reasonably good solutions. For this instance size variant 1 performs best on average with an optimality gap of $2.1 \%$, closely followed by variant 3 with a gap of $2.4 \%$, variant 2 under performs with an average deviation of $5.9 \%$. A comparison with larger instances is not possible due to significantly longer run times. 


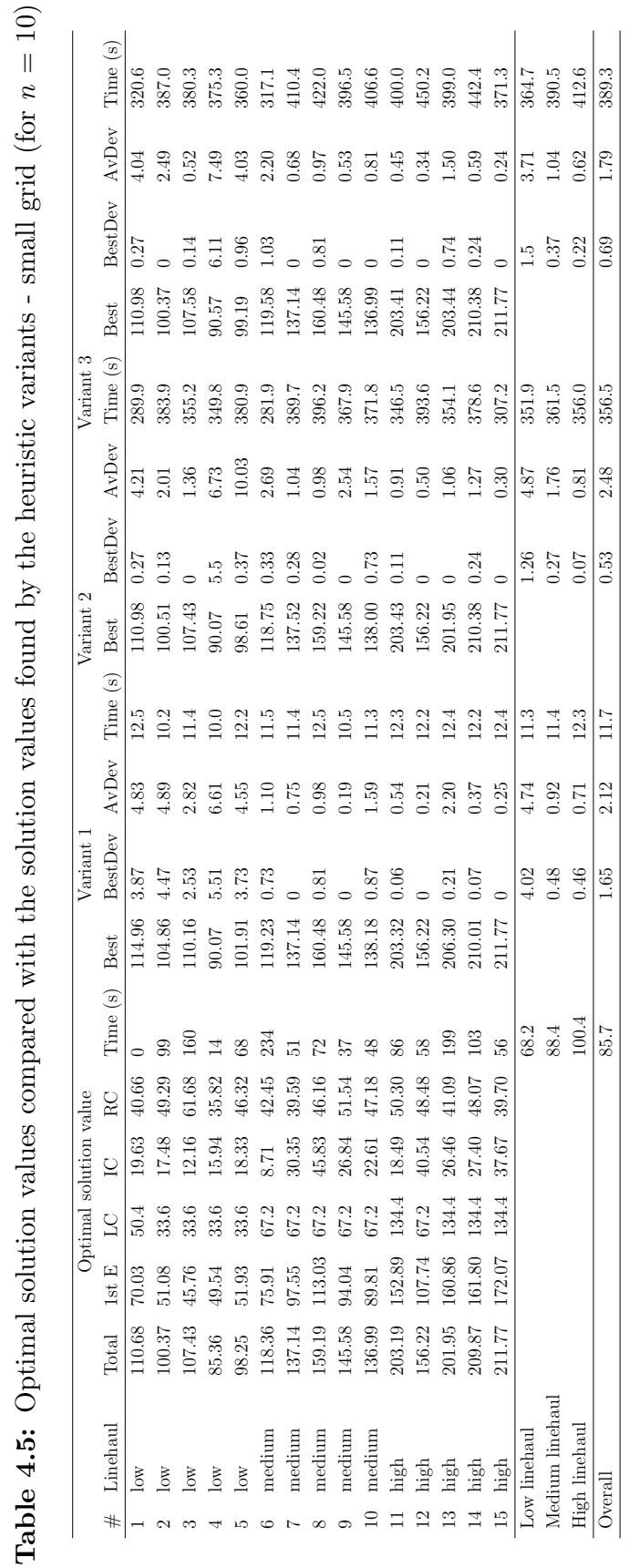




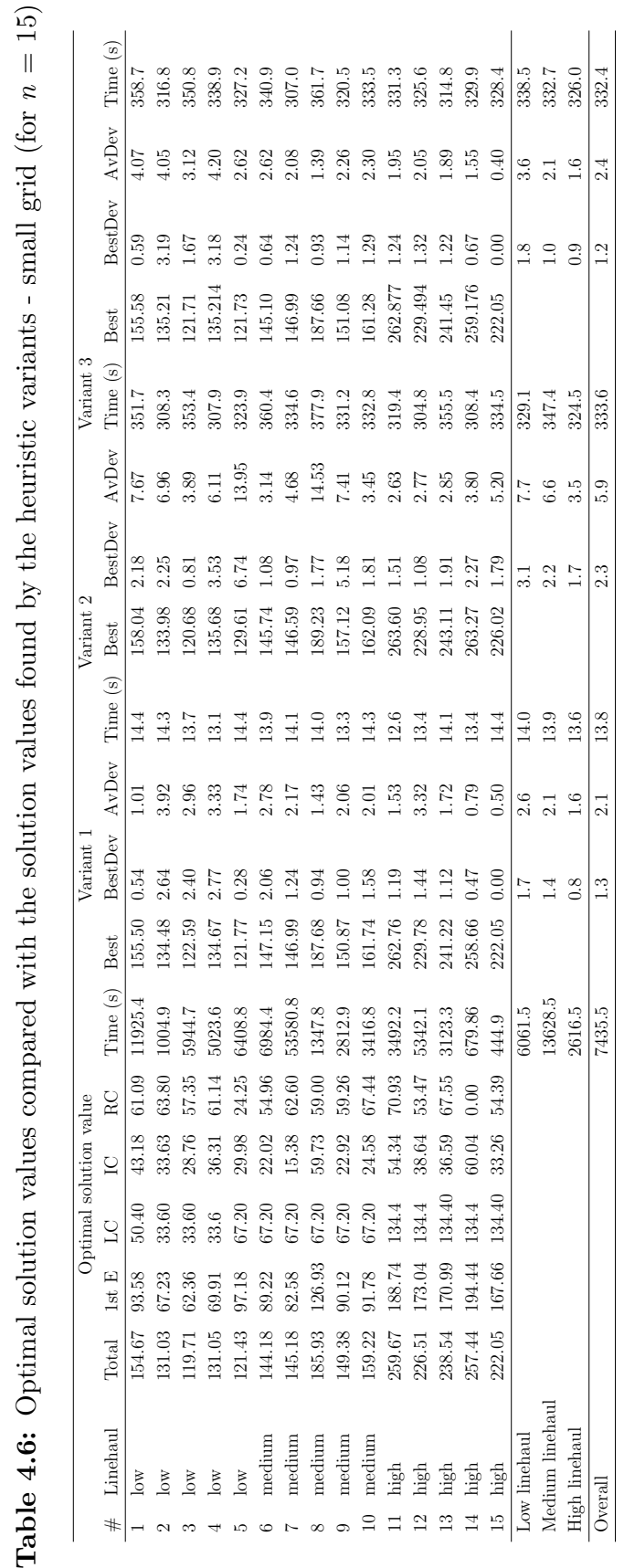


For the large grid instances, the comparison shows that it is harder for the heuristic variants to find optimal solutions. Variant 2 still performs best at finding good objective values, the best being on average only $1.45 \%$ worse than the optimal ones. Variant 3 closely follows with its best objective values being on average $1.64 \%$ worse than the optimal one, while the cost of the best solutions found by variant 1 deviate on average by $6.52 \%$ from those of the optimal solution. Overall, variant 3 performs best with an average optimality gap of $4.52 \%$, followed by variant 2 with a gap of $4.63 \%$, and variant 1 with a gap of $8.41 \%$. This underperformance of variant 1 is caused by its structure which decomposes the problem into the first echelon and a routing problem. Starting by solving the first echelon problem to optimality, the variant fixes the customer delivery patterns and therefore restricts the solution space of the ALNS solutions to the conditional routing problem.

To better quantify this behaviour, Table 4.8 makes a comparison between three algorithmic strategies. The first one, in the left block, solves the problem optimally by CPLEX. The table reports the total cost and its decomposition into its various components. The second strategy, in the middle block, decomposes the problem into its two natural components: the first echelon problem and the routing problem conditioned by the first-echelon solution. It solves each of these two components optimally by CPLEX. The solution values obtained by means of this decomposition strategy deviate on average by $5.97 \%$ from the optimal solution values, even though each component is solved optimally. The third strategy, in the right block, solves the problem by our heuristic variant 1: the first echelon component is again solved optimally, but the routing component is solved heuristically by ALNS. The solution costs obtained under this strategy deviate on average by $7.96 \%$ from the optimal solution values, but only by $1.87 \%$ from the costs obtained under the second strategy. In other words, these results show that ALNS yields good solutions when compared with the optimal values yielded by the second strategy. The deviations observed between variant 1 and the optimal solutions are mostly a result of the decomposition of the problem into its two components, rather than a result of the behaviour of the ALNS per se. Our recommendation is to apply variant 3 and not variant 1 when the cost is relatively important with respect to the total cost. 


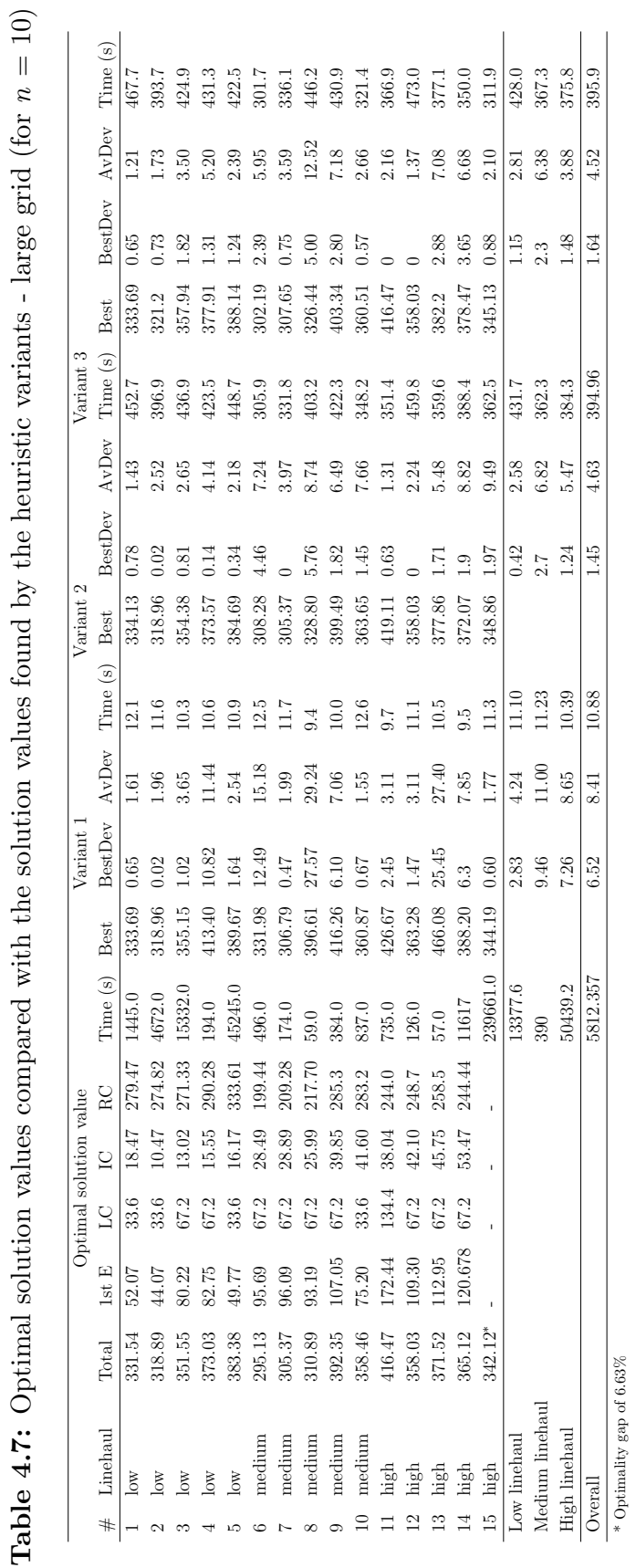




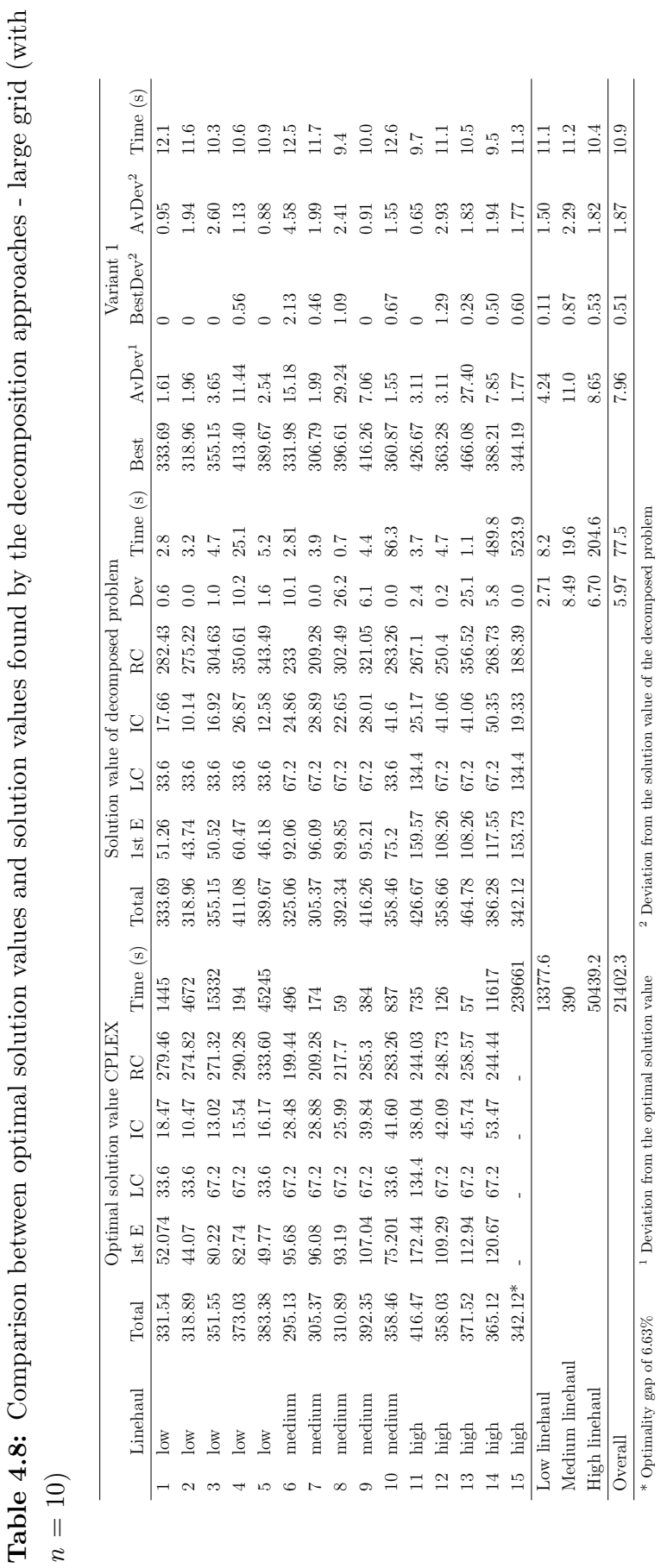




\section{A Two-Echelon Inventory-Routing Problem for Perishable Products}

The results for larger instances are shown in Tables 4.9 and 4.10. The column headings present the different instance size and linehaul cost for the two instance structures and the performance in terms of time and deviation for each heuristic variant. The deviations in the table refer to average percentage deviations from the best solution values found for each of the instances. The percentage in brackets for variant 2 is the average percentage deviation of the final linehaul cost found by variant 2 from the optimal linehaul solution found by the MILP of variant 1 .

Overall, heuristic variants 2 and 3 show a significantly longer run time than variant 1 due to the destruction of customer patterns during the process of the ALNS and the associated resolution of the MILP formulation. The detailed results in Table 4.9 show that for the instances with a small grid size the first variant performs the best in terms of finding good solutions, followed by variant 3. Both of these variants improve the initial solution by about $30 \%$, with an overall average deviation from the best found solutions of about $1.7 \%$ (variant 1) and $2.93 \%$ (variant 3 ). Breaking down the results for the different linehaul costs indicates, that both variant 1 and 3 perform better for instances with higher linehaul cost. Despite improving its initial solution on average also by about $30 \%$, heuristic variant 2 significantly underperforms in comparison to the other two variants with an average deviation from the best found solutions of about $6.95 \%$. As the cost of the 1st echelon (i.e the linehaul part) of the problem accounts on average for about $44 \%$ of the total cost this underperformance seems closely related to the inability of variant 2 to reach better first echelon solutions, with the first echelon solutions found by variant 2 being $8.9 \%$ worse than the optimal solutions found by the MILP model of variant 1 . 
Table 4.9: Average results by heuristic variant for small grid instances

\begin{tabular}{|c|c|c|c|c|c|c|c|c|}
\hline \multicolumn{2}{|c|}{ Instance Structure 1} & \multicolumn{2}{|c|}{ Variant 1} & \multicolumn{3}{|c|}{ Variant 2} & \multicolumn{2}{|c|}{ Variant 3} \\
\hline Size & Linehaul cost & Deviation (\%) & Time (s) & Devi & $\operatorname{tion}(\%)$ & Time (s) & Deviation $(\%)$ & Time (s) \\
\hline \multirow[t]{3}{*}{30} & low & 2.01 & 20.15 & 9.18 & $(13.84)$ & 256.08 & 2.89 & 235.71 \\
\hline & medium & 1.50 & 19.76 & 7.13 & $(9.29)$ & 273.00 & 2.81 & 265.72 \\
\hline & high & 1.44 & 20.35 & 7.53 & $(7.09)$ & 291.06 & 1.73 & 275.43 \\
\hline \multirow[t]{3}{*}{40} & low & 2.09 & 23.42 & 7.50 & $(10.28)$ & 215.30 & 3.16 & 199.95 \\
\hline & medium & 1.34 & 23.58 & 8.05 & $(11.15)$ & 226.30 & 2.33 & 219.50 \\
\hline & high & 1.03 & 23.75 & 7.67 & $(7.58)$ & 230.44 & 1.81 & 209.62 \\
\hline \multirow[t]{3}{*}{50} & low & 3.27 & 29.55 & 6.27 & (10.99) & 208.00 & 5.56 & 202.66 \\
\hline & medium & 1.81 & 29.48 & 8.04 & $(12.31)$ & 219.54 & 2.52 & 208.40 \\
\hline & high & 1.24 & 30.00 & 6.94 & $(8.52)$ & 213.35 & 2.06 & 201.29 \\
\hline \multirow[t]{3}{*}{100} & low & 2.23 & 52.66 & 6.50 & $(10.59)$ & 201.97 & 4.45 & 198.41 \\
\hline & medium & 1.51 & 53.07 & 7.29 & $(9.07)$ & 209.19 & 3.35 & 198.83 \\
\hline & high & 1.02 & 53.42 & 5.69 & $(5.57)$ & 216.60 & 2.58 & 204.91 \\
\hline \multirow[t]{3}{*}{150} & low & 1.73 & 86.90 & 5.04 & $(6.16)$ & 196.52 & 3.14 & 198.48 \\
\hline & medium & 1.72 & 85.14 & 5.54 & $(6.94)$ & 200.91 & 2.87 & 204.00 \\
\hline & high & 1.18 & 84.92 & 5.88 & $(6.69)$ & 204.56 & 2.19 & 212.45 \\
\hline \multicolumn{2}{|c|}{ Low linehaul } & 2.27 & 42.54 & 6.90 & $(10.37)$ & 215.57 & 3.84 & 207.04 \\
\hline \multicolumn{2}{|c|}{ Medium linehaul } & 1.58 & 42.21 & 7.21 & $(9.75)$ & 225.79 & 2.78 & 219.29 \\
\hline \multicolumn{2}{|c|}{ High linehaul } & 1.18 & 42.49 & 6.74 & $(7.09)$ & 231.20 & 2.07 & 220.74 \\
\hline \multicolumn{2}{|c|}{ Overall } & 1.67 & 42.41 & 6.95 & $(9.07)$ & 224.19 & 2.90 & 215.69 \\
\hline
\end{tabular}

The detailed results for the second instance structure with a larger grid size are presented in Table 4.10. It can be seen, that the difference in performance between the three heuristic variants is not as large as for the first set of instances. Variant 1 performs best with respect to the quality of the solutions found, with an average deviation from the best solution values of $4.66 \%$, followed by variant 3 with a deviation of $5.75 \%$. Heuristic variant 2 performs only slightly worse than the other two variants with an average deviation of $6.43 \%$. The average deviations are higher than for instance structure 1 , but the improvement from the initial solutions is also larger, with an improvement from the initial solution by all three variants of about $41 \%$. When distinguishing between different linehaul costs, the results show that all variants perform better for instances with high linehaul costs. While variant 2 still underperforms in terms of finding good solutions for the first echelon of the problem (with a deviation of $10.46 \%$ ), 1st echelon costs only account for about $20 \%$ of the total costs. Thus, it partly compensates for the larger linehaul cost by allowing for more flexibility and finding better solutions for the routing part of the problem. This suggests, that the ratio between linehaul and routing costs impacts the performance of the heuristic variants. 
Table 4.10: Average results by heuristic variant for large grid instances

\begin{tabular}{|c|c|c|c|c|c|c|c|c|}
\hline \multicolumn{2}{|c|}{ Instance Structure 1} & \multicolumn{2}{|c|}{ Variant 1} & \multicolumn{3}{|c|}{ Variant 2} & \multicolumn{2}{|c|}{ Variant 3} \\
\hline Size & Linehaul cost & Deviation (\%) & Time (s) & Devi & tion $(\%)$ & Time $(s)$ & Deviation (\%) & Time (s) \\
\hline \multirow[t]{3}{*}{30} & low & 6.44 & 20.15 & 8.88 & $(13.53)$ & 184.92 & 7.63 & 179.94 \\
\hline & medium & 5.00 & 19.96 & 5.33 & $(14.67)$ & 184.08 & 4.78 & 183.00 \\
\hline & high & 4.35 & 19.89 & 6.96 & $(11.70)$ & 193.27 & 5.68 & 184.82 \\
\hline \multirow[t]{3}{*}{40} & low & 6.40 & 25.18 & 6.47 & $(12.81)$ & 166.42 & 7.53 & 174.02 \\
\hline & medium & 6.85 & 25.48 & 7.36 & $(14.75)$ & 167.12 & 8.45 & 174.52 \\
\hline & high & 3.72 & 25.30 & 5.40 & $(8.27)$ & 182.47 & 4.77 & 180.84 \\
\hline \multirow[t]{3}{*}{50} & low & 6.16 & 28.00 & 6.76 & $(9.28)$ & 157.69 & 6.83 & 163.01 \\
\hline & medium & 6.20 & 28.76 & 6.29 & $(12.71)$ & 163.82 & 6.52 & 167.10 \\
\hline & high & 4.51 & 29.41 & 7.32 & $(13.81)$ & 166.85 & 5.42 & 165.15 \\
\hline \multirow[t]{3}{*}{100} & low & 3.41 & 51.41 & 6.03 & $(11.78)$ & 177.38 & 4.78 & 175.16 \\
\hline & medium & 3.50 & 50.11 & 6.14 & $(7.84)$ & 180.20 & 5.86 & 176.66 \\
\hline & high & 2.66 & 50.16 & 5.63 & $(7.42)$ & 179.17 & 3.81 & 176.05 \\
\hline \multirow[t]{3}{*}{150} & low & 3.34 & 81.84 & 4.81 & $(5.22)$ & 196.83 & 4.45 & 198.36 \\
\hline & medium & 3.94 & 81.86 & 7.22 & $(7.19)$ & 200.69 & 5.46 & 202.50 \\
\hline & high & 3.39 & 80.50 & 5.87 & $(5.87)$ & 203.12 & 4.31 & 201.64 \\
\hline \multicolumn{2}{|c|}{ Low linehaul } & 5.15 & 41.32 & 6.59 & $(10.52)$ & 176.65 & 6.25 & 178.10 \\
\hline \multicolumn{2}{|c|}{ Medium linehaul } & 5.10 & 41.23 & 6.47 & (11.43) & 179.18 & 6.21 & 180.76 \\
\hline \multicolumn{2}{|c|}{ High linehaul } & 3.72 & 41.05 & 6.24 & $(9.41)$ & 184.97 & 4.80 & 181.70 \\
\hline \multicolumn{2}{|c|}{ Overall } & 4.66 & 41.20 & 6.43 & (10.46) & 180.27 & 5.75 & 180.18 \\
\hline
\end{tabular}

In addition, testing instances (of 30 to 50 customers), in which more delivery patterns are allowed per customer, has shown that for both instance structures the differences in performance between the three heuristic variants become more pronounced as the number of delivery patterns increases.

\subsection{Conclusion}

We have introduced the two-echelon inventory routing problem for perishable products. The problem was formulated mathematically and was solved by applying a two-stage matheuristic combining an ALNS with a MILP formulation. Three variants of the matheuristic were proposed and tested on different types of instance structures, varying in grid size and linehaul cost. The results demonstrate that instances of realistic sizes (involving up to 150 customers) can be solved by means of the proposed heuristic within reasonable computing times. The three variants of the heuristic differ greatly on small grid instances, but tend to become more similar on the larger grid instances. It is also easier to solve the problem optimally on the smaller grids. One limitation of this chapter, which could possibly be overcome in future research, lies in the modelling of perishabil- 


\section{Operational Aspects}

ity. Indeed, we have assumed in our model that all products deteriorate linearly as a function of time. However, these phenomena are more complex in practice since not all products deteriorate linearly and at the same rate. Therefore, a more refined model could be exploited, particularly one that would take stochasticity into account. 


\section{Chapter 5}

\section{Operational Aspects}

Last mile distribution with delivery options for fresh products: A location-routing problem with time-windows 


\section{Abstract}

This research presents a location-routing problem with delivery options for the last mile delivery of fresh products. Product delivery can occur either directly to the customer locations or indirectly to a customer pickup point, where they are stored until customer pickup takes place. Due to the fresh nature of the products, direct delivery requires customer attendance and is thus subject to tight time windows at the customer locations, whereas indirect delivery allows for more flexibility. However, pickup points are restricted in terms of capacity and require refrigeration, therefore incurring a cost related to the operation and cooling of the pickup facilities in use. The objective is to minimise the total transportation and storage cost. Formulating the problem as a mixed integer linear program and solving it by means of an adaptive large neighbourhood search, the research aims to investigate the potential benefits of implementing refrigerated pickup stations in last mile distribution systems for fresh products. Results for the computational experiments on a set of benchmark instances indicate potential of introducing pickup stations to reduce the total cost associated with the delivery. 


\subsection{Introduction}

The rapid growth in e-commerce and e-grocery businesses has led to a growing demand for logistic solutions in the context of last mile delivery. However, last mile distribution poses many challenges due to limited customer availability, dispersed customer locations and small customer demands (Zhou et al., 2018). The last mile is thus often regarded as one of the most costly and inefficient stages in the supply chain (Gevaers et al., 2009). This is particularly true for the delivery of highly perishable products, such as fresh food or flowers, that require cold chain technology and special handling of products in order to prevent product loss and quality decay (Akkerman et al., 2010; Morganti et al., 2014). Failed deliveries are a common occurrence in last mile logistics generating additional delivery efforts that lead to higher operating costs (Arnold et al., 2018). Customer pickup points, such as parcel lockers or small intermediary depots, where customers can pick up their goods, e.g. on the way from work or close to home, provide an attractive alternative to standard home delivery, improving efficiency while keeping customer satisfaction levels high. Especially in the case of e-groceries, where 1) delivery time windows are often tight, 2) delivery to neighbours is generally not an option due to the perishable nature of products and 3) failed delivery may not be accepted by customers, these kind of solutions hold potential to ensure smooth operations. However, in the case of perishable products these pickup facilities also need to be refrigerated and thus require energy for cooling, resulting in additional costs and emissions Bozorgi et al. (2014). Efficient delivery planning, thus plays an important role in the last mile distribution of fresh products and can help to reduce product spoilage, cut costs and improve the environmental impact of the "last mile".

\subsubsection{Literature background}

The topic of vehicle routing problems (VRPs), concerned with the improvement of delivery operations, has been extensively studied in the scientific literature. A general overview of the field and the developments over the past decades can be found in Cordeau et al. (2007), Laporte (2009) as well as Toth and Vigo (2014). The joint study of the often interdependent facility location and vehicle routing decisions, known as the Location-Routing Problem (LRP), is an important variant of the general VRP (Albareda-Sambola, 2015; Drexl and Schneider, 2015; Prodhon and Prins, 2014; Nagy and Salhi, 2007) with many applications in city logistics (Boudoin et al., 2014) (Mancini et al., 2014; Sterle, 2010). These kind of studies often focus on two-echelon LRPs (Contardo et al., 2012; Nguyen et al., 2012; Schwengerer et al., 2012; Crainic et al., 2011), mostly excluding temporal aspects such as time windows (Drexl and Schneider, 2015). Proposing a location-routing 


\section{Operational Aspects}

problem with time windows under uncertainty, Zarandi et al. (2013) assume demand and travel times to be fuzzy and solve the problem using simulated annealing. Govindan et al. (2014) incorporate time windows into their multi-objective location-routing problem for the sustainable design of a food supply network. The problem is solved using a hybrid approach based on an adaptive large neighbourhood search (ALNS). Focusing on vehicle emissions in city logistics, Koç et al. (2016) present a model to investigate the impact of location, fleet and routing decisions and solve it using an ALNS approach.

The study of city logistics holds particular challenges for the optimal routing of vehicles and has hence received growing attention in the scientific literature of the past decades (Cattaruzza et al., 2017; Bektas et al., 2017; Savelsbergh and Van Woensel, 2016; Crainic et al., 2009). The design and operation of pick-up point networks provide opportunities in this context, but has received so far only little attention within the scientific literature (Savelsbergh and Van Woensel, 2016). Considering a two-echelon setting with multiple depots, Zhou et al. (2018) are the first to propose a vehicle routing problem with delivery options for the last mile distribution of e-commerce. The problem is solved using a hybrid multi-population genetic algorithm and tested for real life instances. Other problems that can be related to the optimisation of delivery options include the covering tour VRP (such as Karaoğlan et al. (2018), Flores-Garza et al. (2017); Nedjati et al. (2017); Pham et al. (2017); Allahyari et al. (2015); Jozefowiez (2014) and Ha et al. (2013)) and the ring-star problem (e.g. Baldacci et al. (2017); Hill and Voß (2016) as well as Baldacci and Dell'Amico (2010)). Moreover, the vertices of a cluster in the generalised VRP (adressed by, for example, Ha et al. (2014); Pop et al. (2012); Bektaş et al. (2011) and Baldacci et al. (2010)) can be seen as alternative delivery options. These studies do ,however, not yet address issues related to time windows in the context of alternative delivery locations. For a general overview of the vehicle routing problem with time windows the reader is referred to Cordeau et al. (2002).

\subsubsection{Contribution and organisation of this chapter}

This chapter focuses on the last mile distribution of fresh products under consideration of delivery time windows and alternative delivery locations, where customers can either be served directly or through customer pickup points. In this context, we propose a locationrouting problem with time windows and pickup point considerations to investigate the impact of these design and operational decisions on distribution costs and emissions. To our knowledge the problem has not yet been addressed in the scientific literature. We model the problem mathematically and solve it heuristically through an adaptive large neighbourhood search (ALNS).

The remainder of the chapter is organised as follows. In Section 5.2 the problem will be formally described. Section 5.3 introduces the mathematical formulation of the problem 
and Section 5.4 describes the heuristic. Computational results are presented in Section 5.5 and we conclude the chapter in Section 5.6.

\subsection{Formal Problem Description}

We consider the location-routing problem with time windows and alternative delivery options for the last mile delivery of fresh products. We therefore assume a system consisting of a supplier, refrigerated customer pickup points and several customer locations. In this system, a customer can be served either directly, through attended delivery at the customer location, or indirectly, through delivery at a customer pickup point. The solution must satisfy all customer demands. Customer demands are assumed to be deterministic and known in advance. Direct delivery requires customer attendance and thus depends on the availability of the customer, which is provided in the form of time windows during which delivery can occur. Indirect delivery at the pickup points on the other hand can be made at any time before a certain due date prior to the collection time window of the customer. Multiple collection time windows, over the course of a day, and thus re-stocking of the delivery points are possible. Delivery points are, however, restricted in terms of their capacity and require refrigeration during storage to prevent deterioration of the fresh products delivered to the pickup points. Storage therefore incurs a cost related to the operation and cooling of the used pickup facility modules. Figure 5.1 gives a schematic overview of the described system. Delivery is carried out from the supplier to the final delivery location using a fleet of homogeneous vehicles. Vehicles are readily available at the supplier's location, however, restricted in terms of their capacity. The problem consists of the following decisions:

- Which delivery option to choose for a customer?

- How many and which pickup locations and storage modules to open?

- When to deliver items for customer pickup?

- What is the optimal routing to the customer locations and pickup points?

The aim is therefore to determine the optimal delivery locations and routing to serve all customers, and optimise the delivery schedule and capacity use at the pickup facilities, minimising both the routing and storage/cooling costs. 


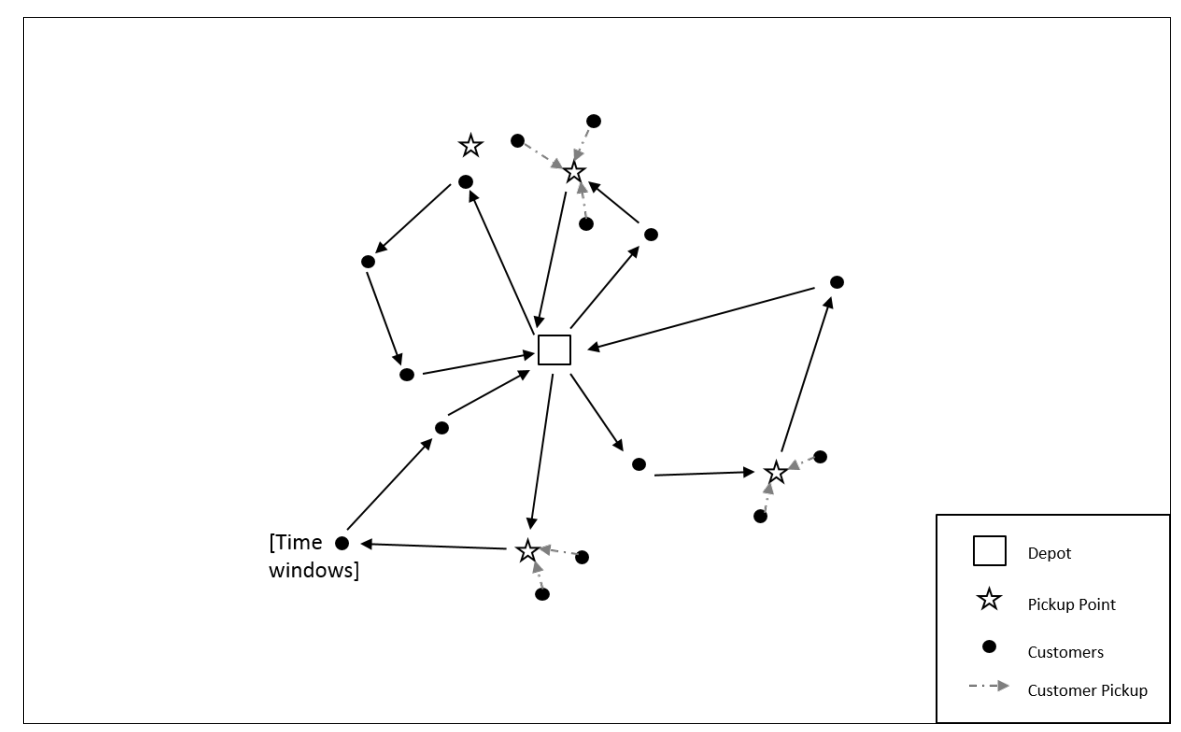

Figure 5.1: Delivery system with pickup points

\subsection{Mathematical Formulation}

This section introduces the notations and parameters used and provides a formal description of the mixed integer linear programming (MILP) formulation based on the assumptions presented in Section 5.2. 
Table 5.1: Summary of notation

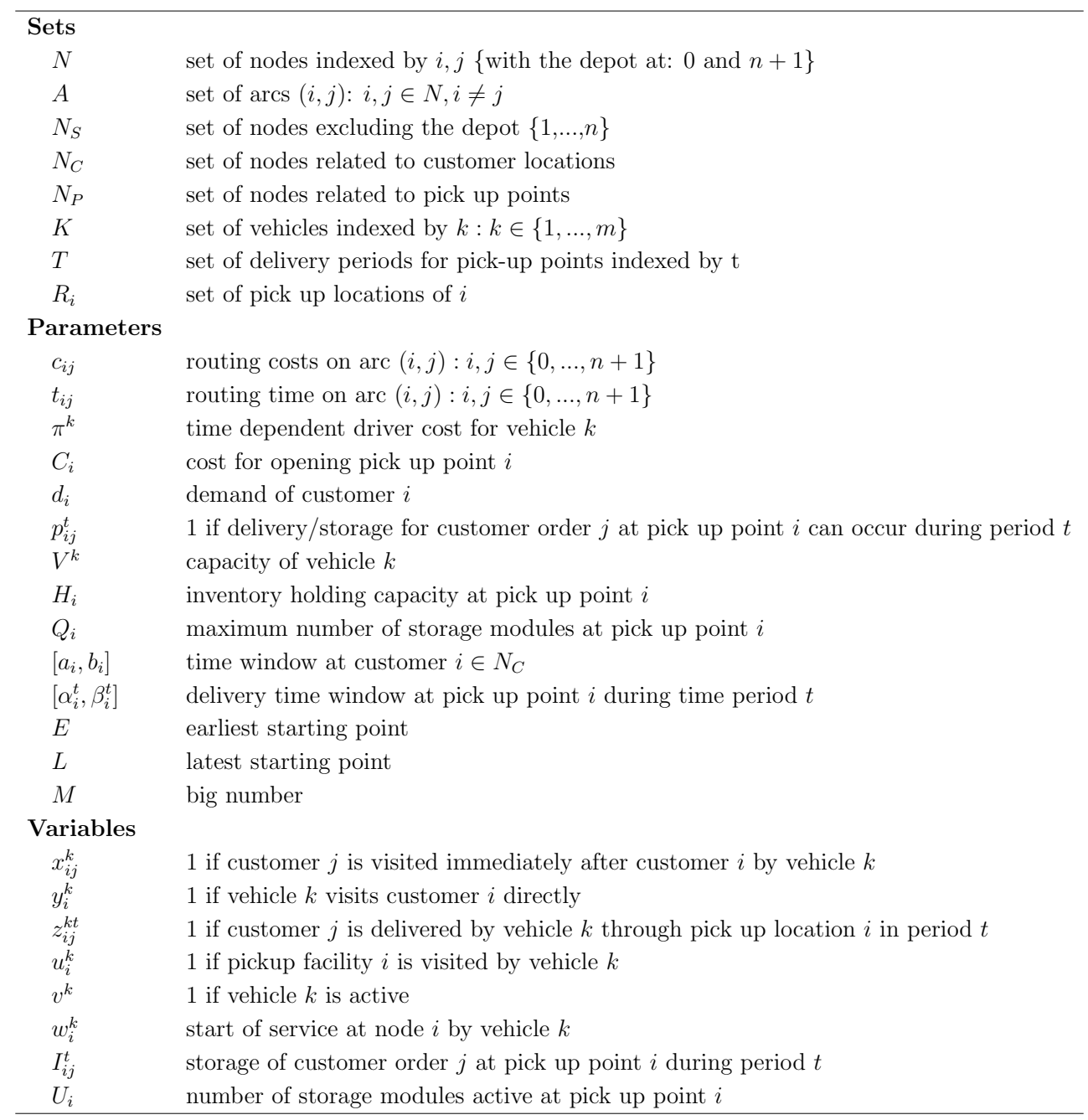

The problem is then:

Minimise $\quad \sum_{i \in N} \sum_{j \in N} \sum_{k \in K} c_{i j} x_{i j}^{k}+\sum_{k \in K} \pi^{k}\left(w_{n+1}^{k}-w_{0}^{k}\right)+\sum_{i \in N_{P}} C_{i} U_{i}$ 
subject to

$$
\begin{aligned}
& \sum_{j \in N_{S}} x_{0 j}^{k}=\sum_{i \in N_{S}} x_{i, n+1}^{k}=v^{k} \quad k \in K \\
& v^{k} \geq v^{k+1} \quad k \in\{1, \ldots, m-1\} \\
& \sum_{k \in K} y_{j}^{k}+\sum_{i \in R_{j}} \sum_{k \in K} \sum_{t \in T} z_{i j}^{k t}=1 \quad j \in N_{C} \\
& \sum_{i \in N_{P}} \sum_{j \in N_{C}} \sum_{t \in T} d_{j} z_{i j}^{k t}+\sum_{j \in N_{C}} d_{j} y_{j}^{k} \leq V^{k} v^{k} \quad k \in K \\
& y_{i}^{k} \leq \sum_{j \in N} x_{i j}^{k} \quad i \in N_{C}, k \in K \\
& \sum_{i \in N} x_{i j}^{k}-\sum_{l \in N} x_{j l}^{k}=0 \quad k \in K, j \in N_{S} \\
& w_{i}^{k}+t_{i j}-w_{j}^{k} \leq\left(1-x_{i j}^{k}\right) M \quad i \in N, j \in N, k \in K \\
& E \leq w_{0}^{k} \leq L \quad k \in K \\
& w_{0}^{k} \leq w_{n+1}^{k} \quad k \in K \\
& a_{i} y_{i}^{k} \leq w_{i}^{k} \leq b_{i} y_{i}^{k} \quad i \in N_{C}, k \in K \\
& \alpha_{i}^{t} p_{i j}^{t} z_{i j}^{k t}+M\left(z_{i j}^{k t}-u_{i}^{k}\right) \leq w_{i}^{k} \leq \beta_{i}^{t} p_{i j}^{t} z_{i j}^{k t}+M\left(u_{i}^{k}-z_{i j}^{k t}\right) \quad i \in N_{P}, j \in N_{C}, k \in K, t \in T \\
& w_{i}^{k} \leq v^{k} M \quad i \in N, k \in K \\
& u_{i}^{k} \leq \sum_{j \in N} x_{i j}^{k} \quad i \in N_{P}, k \in K \\
& \sum_{j \in N_{C}} I_{i j}^{t} \leq H_{i} U_{i} \quad i \in N_{P}, t \in T \\
& U_{i} \leq Q_{i} \quad i \in N_{P} \\
& I_{i j}^{0}=\sum_{k \in K} d_{j} z_{i j}^{k 0} \quad i \in N_{P}, j \in N_{C} \\
& I_{i j}^{t}=p_{i j}^{t} I_{i j}^{t-1}+\sum_{k \in K} d_{j} z_{i j}^{k t} \quad i \in N_{P}, j \in N_{C}, t \in T \backslash\{0\} \\
& w_{i}^{k}, I_{i j}^{t}, \geq 0 \\
& x_{i j}^{k}, y_{i}^{k}, z_{i j}^{k t}, u_{i}^{k}, v^{k} \in\{0,1\} \\
& U_{i} \in \mathbb{Z} \text {. }
\end{aligned}
$$

The objective function (5.1) minimises the sum of the delivery cost, consisting of personnel cost and routing cost, and the cost of operating the pick up points. Constraints (5.2) ensure that each active vehicle starts and ends its route at the depot, while constraints (5.3) establish the order according to which vehicles become active. Constraints (5.4) state that each customer order is delivered either directly or through a pick up facility. Constraints (5.5) impose a vehicle capacity that cannot be exceeded. Constraints (5.6) 
mean that a direct delivery can only be made if the customer is visited by an active vehicle. Constraints (5.7) are flow conservation constraints. Constraints (5.8-5.14) guarantee schedule feasibility with respect to time considerations. Constraints (5.15-5.18) define the inventory at the pick up points and restrict the amount that can be delivered to the facilities during each period based on the inventory capacity of the available storage modules. Constraints (5.19-5.21) enforce the non-negativity and integrality of the variables.

\subsection{Heuristic}

Given that it is already difficult to solve the problem to optimality for small instances by a standard integer linear programming solver, an adaptive large neighbourhood search (ALNS) framework is proposed to solve the problem for larger instances. The basic structure of the approach is described in pseudo-code in Algorithm 7, for a more detailed overview of the ALNS approach we refer to Ropke and Pisinger (2006). The focus in the following will be on the customised components of the ALNS, such as the construction of the initial solution and the problem specific destroy and repair operators.

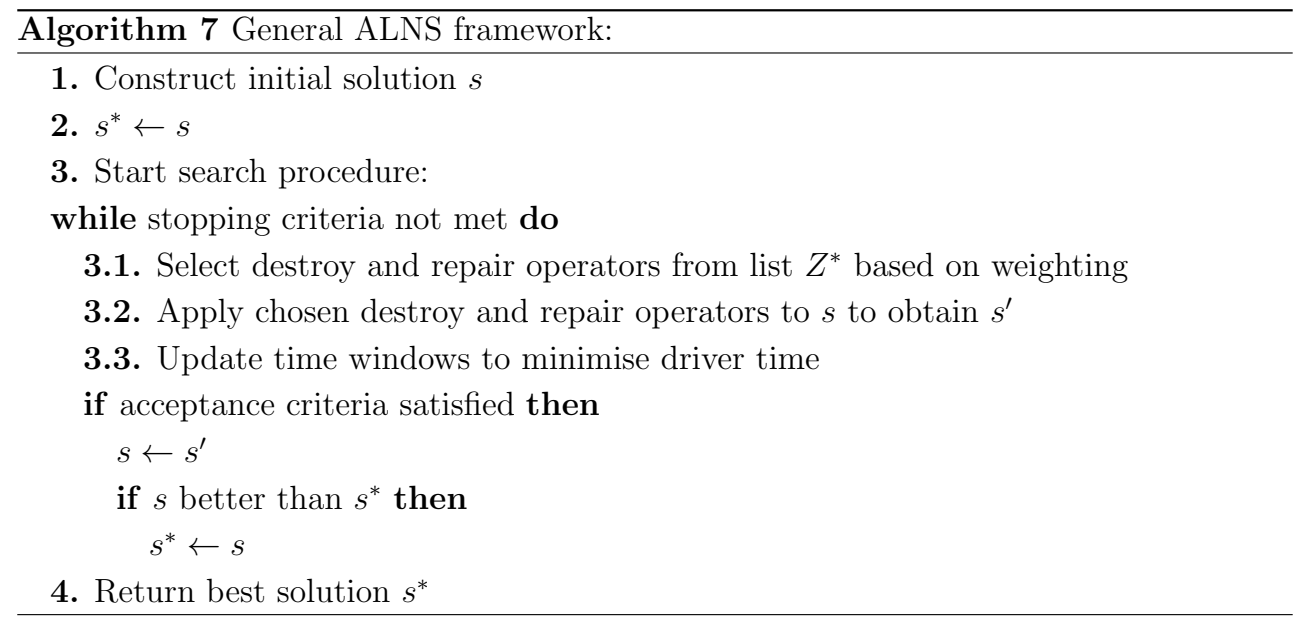

\subsubsection{Construction of an initial solution}

Focusing solely on direct delivery to the customer locations, an initial routing solution is constructed using a best insertion algorithm. Possible insertions include all the insertions in existing routes, based on feasibility with regards to time window restrictions of a customer and available vehicle capacity, and insertions by creation of a new route. The procedure is completed once all customers have been allocated to a route. 


\subsubsection{Destroy operators}

We propose a number of different destroy operators, operating at the customer, pick-up station and route level. Once an operator is applied it removes a percentage $\mathrm{Nb}$ of the customers from the current solution.

Random Customer Removal: In this standard operator customers are picked at random and removed from the current solution.

Worst Customer Removal: Customers are evaluated based on their insertion cost and the worst customers, i.e. the customers with the largest insertion costs, are removed from the solution.

Related Customer Removal: Based on the related customer removal operators used by Shaw (1998) and Azi et al. (2014), this operator chooses related customers by means of a proximity measure and removes them from the solution. The structure of the operator is described in Algorithm 8.

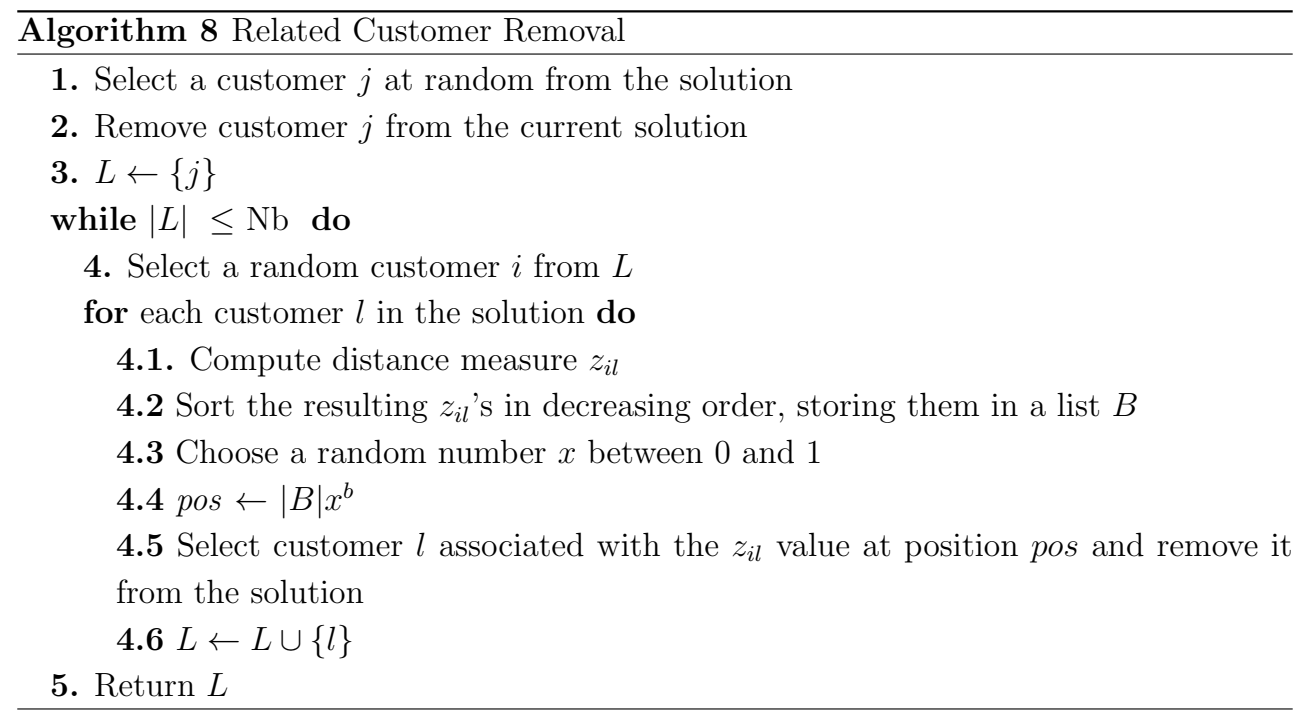

Two variants of the operator are applied. The first one uses a pure spatial distance measure given by $\delta_{i l}=c_{i l}$. The second one applies a measure defined by both spatial and temporal distance similar to Azi et al. (2014), so that $\delta_{i l}=\lambda c_{i l}+(1-\lambda) \mid a_{i}-$ $a_{l} \mid$. The parameter $\mathrm{b}$ at step 5.4 determines the intensity of the bias towards closer customers, tuning this parameter resulted in a setting of $b=10$ in our implementation of the heuristic. 
Random Route Removal: A number of routes are chosen at random and removed from the current solution.

Random Pick-up Module Removal: A number of pick-up modules are chosen at random and removed from the current solution.

\subsubsection{Repair operators}

After having been removed from a solution, customers need to be reinserted by using a repair operator in order to create a new solution. Two kinds of repair operators are considered in this context:

Cheapest Insertion: Following the same procedure as for the construction of the initial solution, a customer is reinserted at the position with the cheapest insertion cost, in accordance with all time-window and capacity restrictions. Two variants are considered, one focusing solely on the insertion of customers into routes and one that also allows for insertion into already open pickup modules.

Pickup Insertion: Customers are reinserted in the solution through alternative delivery at a pickup point by allocating each customer to a pickup location. Taking into account capacity considerations, customers are allocated to a delivery batch. In the case of a new batch, the visit to the pickup location is inserted in the routing of the new solution on the basis of cheapest insertion.

\subsubsection{Acceptance criterion and adaptive mechanism}

Candidate solutions are accepted based on simulated annealing, as described in Ropke and Pisinger (2006), and an adaptive mechanism is applied, updating the weighting of the different destroy and repair operators after a certain number of iterations. The heuristic terminates once a predefined number of iterations has been reached.

\subsection{Computational Results}

A number of computational experiments have been carried out to validate the mathematical formulation of the problem and test the proposed heuristic. The heuristic was coded in Python and all experiments were conducted on a machine equipped with an Intel(R) Xeon(R) X5675 processor running at $3.07 \mathrm{GHz}$. 


\subsubsection{Instance description}

The approach is tested on a first set of small instances, adapting the well-known Solomon instances for the VRP with time windows (Solomon, 1987). The detailed characteristics of the set are described in the following.

\section{Adapted benchmark instances}

The set of instances is generated based on the C1 instances proposed by Solomon (1987), using the same customer locations, demands and time windows. Routing time and cost are assumed to be identical and calculated based on the Euclidean distances between the locations in the plane. Vehicle capacity is kept the same as in the original instance setting. The depot is located at node 0 with the coordinates $(40,50)$, while the pick-up point is considered to be at point $(25,55)$. All customers may be delivered through alternative delivery at the pick-up point. Customer pick-up is assumed to occur any time after the latest starting point of vehicles at the depot, resulting in one delivery time period. The delivery time window at the pick-up point therefore corresponds to the operating time window of the depot, given by $E$ and $L$. Inventory capacity of a storage module at the customer pick-up point is set to $H=150$ for all instances. The cost of operating a storage module is equal to $C=100$ and the maximum number of modules at the pickup location is limited to $q=2$. The time dependent driver cost for a vehicle is set to $\pi=0.5$. A graphical representation of the location setting for the instances of different customer sizes is presented in Figure 5.2; the depot location is indicated by the black marker and the pickup location is highlighted in red.
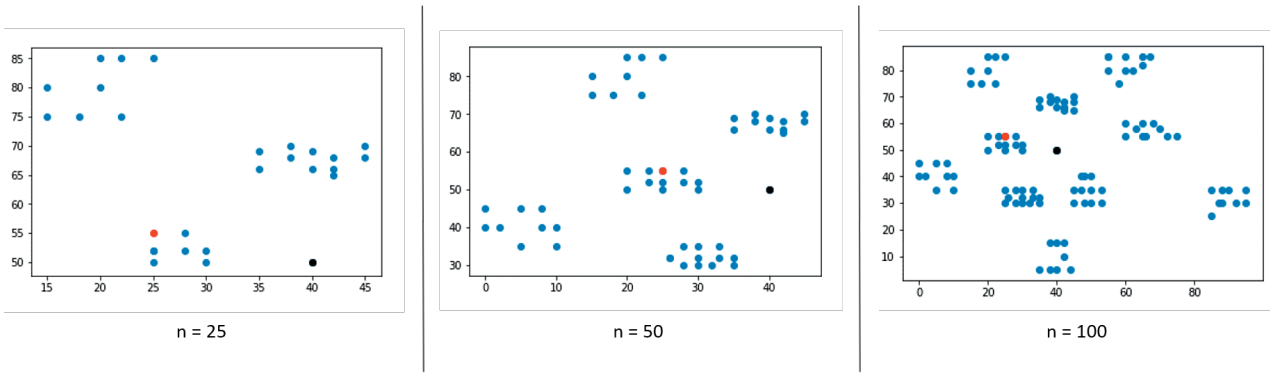

Figure 5.2: Instance settings 


\subsubsection{Parameter settings}

The tuning for the set of instances was carried out using a set of randomly selected test instances, consisting of a total of 9 instances. For each parameter setting, 10 heuristic runs have been executed and the settings with the smallest average deviation from the best found solution was chosen. The search procedure consisted of a total of 25,000 iterations and a segment size of 200 iterations, as this provided a good trade-off between run time and solution quality. For the percentage of destruction (i.e. the percentage of customers to remove from the current solution), the following three intervals were reviewed: $[10 \%, 30 \%],[20 \%, 40 \%]$, [30\%, 50\%]. The values for all other parameters were initially set equal to those of Ropke and Pisinger (2006) and then sequentially altered in the tuning phase. The resulting best parameter setting for the benchmark instances is shown in Table 5.2

Table 5.2: Parameter settings

\begin{tabular}{cc}
\hline Parameters & Benchmark Instances \\
\hline Percentage of destruction: & $10 \%-30 \%$ \\
Acceptance criterion: & \\
$w$ & 0.005 \\
$c$ & 0.99985 \\
Weight adjustment: & \\
$\sigma_{1}$ & 33 \\
$\sigma_{2}$ & 9 \\
$\sigma_{3}$ & 13 \\
$r$ & 0.9 \\
\hline
\end{tabular}

\subsubsection{Results}

This section reports the results for the benchmark instances based on the best parameter settings identified in Section 5.5.2. For the instances with 25 customers, these results are compared with the solution values found by the mathematical model after one hour and after ten hours of run time. The column headings of Table 5.3 present the solutions found by the mathematical model and the remaining optimality gap after one hour and after ten hours of run time as well as performance of the heuristic. The total cost terms are broken down into the different cost components, namely the routing cost $(\mathrm{RC})$, the driver cost (DC) and the cost for the pickup locations (PC). For the heuristic, the table provides the best solution values as well as the performance of the algorithm in terms of time and deviation from the solutions found by the MILP after 10 hours of run time. 


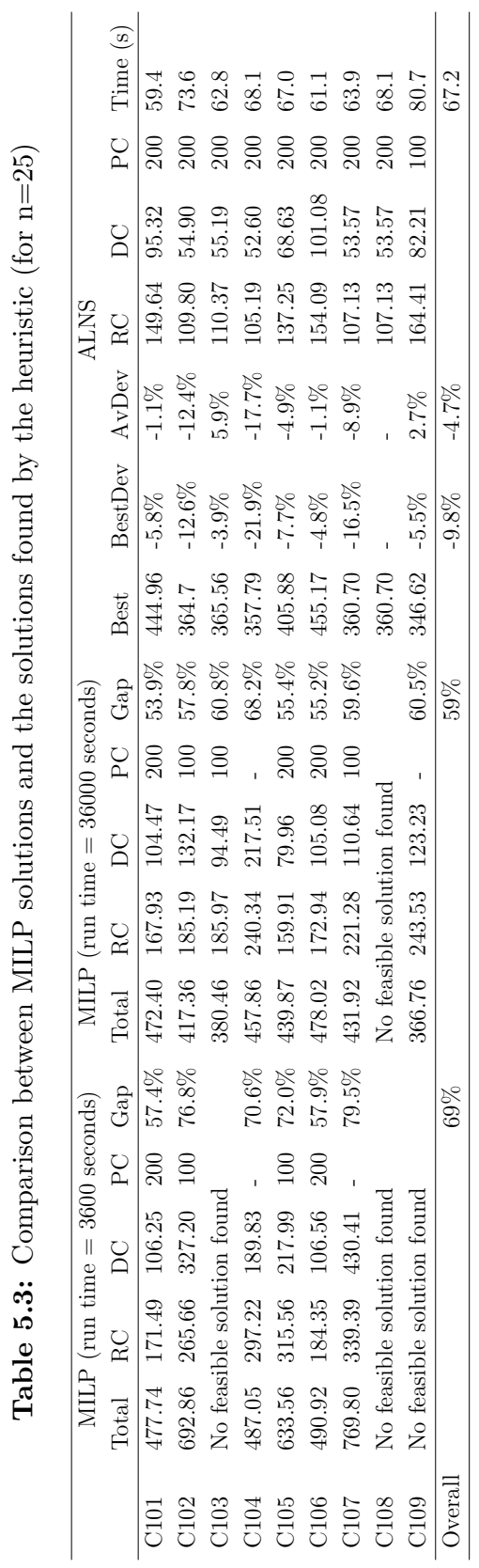


The comparison shows that the heuristic generally outperforms the MILP with restricted run times, finding good solutions in a much shorter time. With an overall average run time of about 70 seconds, the solutions found by the heuristic are on average about $5 \%$ better than the solution found by the MILP after 10 hours of run time. In the best case, the heuristic even finds solutions that are on average about $10 \%$ better, ranging from $4 \%$ up to $22 \%$ lower costs in comparison to the MILP after 10 hours. The analysis further shows that while the MILP struggles in some cases to find feasible solutions within a reasonable time, the heuristic is able to find good solutions for these instances. Despite the worse average performance in comparison to the solutions eventually found by the MILP, it can thus be said that the heuristic still outperforms the MILP in these cases.

Given the poor performance of the MILP for the instances with 25 customers, the comparison was no longer deemed sensible for larger sized instances. Instead, to investigate the potential of using pickup facilities, the solutions of the heuristic with pickup considerations are compared to the best solution values found by a pure routing version of the heuristic. In this context, Tables 5.4 to 5.6 show the performance of the heuristic with pickup in comparison to the best solutions found by a pure routing heuristic. The total cost terms for the best solutions found are again broken down into their components and best and average deviations are computed for the pickup heuristic with respect to the best solution values found by the pure routing heuristic for each of the instances.

Table 5.4: Comparison between pure routing and routing with customer pickup $(n=25)$

\begin{tabular}{|c|c|c|c|c|c|c|c|c|c|c|c|c|}
\hline & \multicolumn{3}{|c|}{ Pure Routing Heuristic } & \multicolumn{9}{|c|}{ ALNS with Pickup } \\
\hline & Best & Routing & Driver & Pickup & Time $(\mathrm{s})$ & Best & BestDev & AvDev & Routing & Driver & Pickup & Time $(\mathrm{s})$ \\
\hline C101 & 808.80 & 463.43 & 345.37 & 0 & 323.0 & 444.96 & $-45.0 \%$ & $-42.2 \%$ & 149.64 & 95.32 & 200 & 59.4 \\
\hline C102 & 608.14 & 303.90 & 304.24 & 0 & 291.3 & 364.70 & $-40.0 \%$ & $-39.9 \%$ & 109.80 & 54.90 & 200 & 73.6 \\
\hline C103 & 523.08 & 300.53 & 222.55 & 0 & 306.2 & 365.55 & $-30.1 \%$ & $-23.0 \%$ & 110.37 & 55.18 & 200 & 62.8 \\
\hline C104 & 470.61 & 287.39 & 183.22 & 0 & 283.8 & 357.78 & $-24.0 \%$ & $-19.9 \%$ & 105.19 & 52.59 & 200 & 68.1 \\
\hline C105 & 609.50 & 376.80 & 232.70 & 0 & 310.5 & 405.88 & $-33.4 \%$ & $-31.4 \%$ & 137.25 & 68.63 & 200 & 67.0 \\
\hline C106 & 792.03 & 422.17 & 369.86 & 0 & 322.2 & 455.17 & $-42.5 \%$ & $-40.3 \%$ & 154.09 & 101.08 & 200 & 61.1 \\
\hline C107 & 478.35 & 288.43 & 189.92 & 0 & 295.0 & 360.70 & $-24.6 \%$ & $-17.7 \%$ & 107.13 & 53.57 & 200 & 63.9 \\
\hline C108 & 422.82 & 267.88 & 154.94 & 0 & 281.2 & 360.70 & $-14.7 \%$ & $-5.0 \%$ & 107.13 & 53.57 & 200 & 68.1 \\
\hline C109 & 383.79 & 255.86 & 127.93 & 0 & 240.3 & 346.62 & $-9.7 \%$ & $-1.9 \%$ & 164.41 & 82.20 & 100 & 80.7 \\
\hline Overall & & & & & 294.8 & & $-29.3 \%$ & $-24.6 \%$ & & & & 67.2 \\
\hline
\end{tabular}

For the instances with 25 customers, the comparison shows that the insertion of pickup locations holds a large potential to improve the routing solution, with the solutions of the pickup heuristic being on average about $25 \%$ better than the pure routing solution values. The best solution values found by the ALNS with pickup considerations even lead to an average improvement of almost 30\%. In addition, the ALNS with pickup also showcases a much shorter run time as less time window feasibility checks are needed, reducing the run time from about 300 seconds for the pure routing to about 70 seconds for the routing with pickup. 


\section{Operational Aspects}

Similar results are shown in Table 5.5 for the instances with 50 customers, depicting an average improvement through the addition of pickup considerations of around $20 \%$. When comparing only the best solution values, the potential is even larger with the best values being on average about $26 \%$ lower than the best values found by the pure routing heuristic. With an increase in the number of customers, the heuristics need significantly longer run times, though the heuristic with customer pickup still shows a considerably reduced run time in comparison with the pure routing heuristic. This may again be attributed to fewer time window feasibility checks during the search procedure.

Table 5.5: Comparison between pure routing and routing with customer pickup $(n=50)$

\begin{tabular}{lllllllllllll}
\hline & \multicolumn{4}{c}{ Pure Routing Heuristic } & \multicolumn{9}{c}{ ALNS with Pickup } \\
& Best & Routing & Driver & Pickup & Time (s) & Best & BestDev & AvDev & Routing & Driver & Pickup & Time (s) \\
\hline C101 & 1793.07 & 1058.03 & 735.05 & 0 & 2220.6 & 1231.23 & $-31.3 \%$ & $-28.9 \%$ & 625.85 & 405.38 & 200 & 753.4 \\
C102 & 1444.86 & 850.13 & 594.73 & 0 & 2100.4 & 870.13 & $-39.8 \%$ & $-34.9 \%$ & 428.12 & 242.01 & 200 & 714.5 \\
C103 & 1234.03 & 751.04 & 482.99 & 0 & 1883.3 & 727.43 & $-41.1 \%$ & $-37.2 \%$ & 351.62 & 175.81 & 200 & 677.2 \\
C104 & 734.62 & 475.90 & 258.72 & 0 & 1535.1 & 669.23 & $-8.9 \%$ & $-5.1 \%$ & 312.82 & 156.41 & 200 & 634.2 \\
C105 & 1427.77 & 902.34 & 525.43 & 0 & 2071.4 & 1009.93 & $-29.3 \%$ & $-25.8 \%$ & 518.07 & 291.85 & 200 & 718.3 \\
C106 & 1595.26 & 954.72 & 640.54 & 0 & 2111.1 & 1096.59 & $-31.3 \%$ & $-25.3 \%$ & 544.47 & 352.12 & 200 & 787.6 \\
C107 & 1137.08 & 725.50 & 411.58 & 0 & 1916.0 & 882.71 & $-22.4 \%$ & $-17.8 \%$ & 454.60 & 228.11 & 200 & 733.4 \\
C108 & 980.98 & 603.83 & 377.15 & 0 & 1729.8 & 813.09 & $-17.1 \%$ & $-11.9 \%$ & 408.73 & 204.36 & 200 & 713.6 \\
C109 & 799.50 & 532.16 & 267.34 & 0 & 1451.6 & 678.14 & $-15.2 \%$ & $-6.0 \%$ & 318.76 & 159.38 & 200 & 635.1 \\
\hline Overall & & & & & 1891.0 & & $-26.3 \%$ & $-21.4 \%$ & & & 707.5 \\
\hline
\end{tabular}

Table 5.6 presents the comparison between pure routing and routing with customer pickup for instances of 100 customers. The results for these instances indicate a smaller potential to improve the routing solution through the insertion of customer pickup facilities, with an average overall improvement of $10.6 \%$ and a best overall improvement of about $17 \%$. This can be explained due to existing storage limitations, so that in comparison only a smaller percentage of the total number of customers can be delivered through the pickup facility.

Table 5.6: Comparison between pure routing and routing with customer pickup $(n=100)$

\begin{tabular}{|c|c|c|c|c|c|c|c|c|c|c|c|c|}
\hline \multirow[b]{2}{*}{ Best } & \multicolumn{3}{|c|}{ Pure Routing Heuristic } & \multicolumn{9}{|c|}{ ALNS with Pickup } \\
\hline & Routing & Driver & Pickup & Time $(s)$ & Best & BestDev & AvDev & Routing & Driver & Pickup & Time (s) & \\
\hline C101 & 4518.94 & 2559.74 & 1959.20 & 0 & 16345.8 & 3677.69 & $-18.6 \%$ & $-13.1 \%$ & 2187.17 & 1290.52 & 200 & 10366.7 \\
\hline C102 & 3707.88 & 2024.07 & 1683.81 & 0 & 14762.6 & 2819.65 & $-24.0 \%$ & $-16.4 \%$ & 1496.25 & 1123.40 & 200 & 9667.6 \\
\hline C103 & 2784.62 & 1665.46 & 1119.15 & 0 & 12826.0 & 2216.90 & $-20.4 \%$ & $-11.8 \%$ & 1242.95 & 773.95 & 200 & 8327.3 \\
\hline C104 & 2122.28 & 1311.91 & 810.37 & 0 & 9747.2 & 1568.39 & $-26.1 \%$ & $-16.5 \%$ & 910.34 & 458.05 & 200 & 6387.5 \\
\hline C105 & 3557.83 & 2241.40 & 1316.43 & 0 & 14451.2 & 2960.42 & $-16.8 \%$ & $-12.0 \%$ & 1733.69 & 1026.73 & 200 & 9465.6 \\
\hline C106 & 3364.36 & 2068.13 & 1296.23 & 0 & 14319.9 & 2861.12 & $-15.0 \%$ & $-11.0 \%$ & 1698.89 & 962.23 & 200 & 9016.2 \\
\hline C107 & 2944.29 & 1838.88 & 1105.41 & 0 & 13198.1 & 2551.10 & $-13.4 \%$ & $-7.9 \%$ & 1513.84 & 837.26 & 200 & 8768.7 \\
\hline C108 & 2461.09 & 1591.05 & 870.04 & 0 & 11886.5 & 2222.57 & $-9.7 \%$ & $-5.1 \%$ & 1247.20 & 775.37 & 200 & 8280.2 \\
\hline C109 & 1947.96 & 1264.54 & 683.42 & 0 & 9740.8 & 1755.57 & $-9.9 \%$ & $-2.0 \%$ & 1036.71 & 518.86 & 200 & 7070.5 \\
\hline Overall & & & & & 13030.9 & & $-17.1 \%$ & $-10.6 \%$ & & & & 8594.5 \\
\hline
\end{tabular}

The results further highlight the poor performance in terms of run time, showcasing 
once again a significant increase in the computational time as the number of customers increases. Overall, the results indicate, however, that the use of customer pickup locations holds potential to reduce the overall costs by reducing both the routing and the driver cost in the system.

\subsection{Conclusion}

We have introduced a location-routing problem with alternative delivery options and time windows for the last mile delivery of fresh products. The problem was formulated mathematically and solved by means of an adaptive large neighbourhood search. The results demonstrate the potential of using pickup facilities for fresh products in the last mile context. Further analysis is needed with regards to the willingness/ percentage of customers to adopt the option of alternative delivery, as this is likely to impact the size of the potential cost reductions that can be achieved. Moreover, cases considering more than one pickup facility should be investigated. The heuristic should furthermore be refined to reduce the overall run times and support a more consistent performance, in order to be able to solve larger sized instances and maintain a good solution quality. Following from this, the intention for future research is to test the heuristic on a set of real world instances related to the last mile distribution of an online meal kit delivery service, operating in the Benelux countries. This will allow to gain real world managerial insights and investigate the potential of customer pickup facilities in a practical setting. 



\section{Chapter 6}

\section{Product Concepts}

\section{A model for composing meat replacers}

This chapter is published as:

Van Mierlo, K., Rohmer, S., \& Gerdessen, J. C. (2017). A model for composing meat replacers: Reducing the environmental impact of our food consumption pattern while retaining its nutritional value. Journal of Cleaner Production, 165, 930-950. 


\section{Abstract}

Current food consumption patterns have a substantial impact on our environment and are thus considered unsustainable. In the context of global warming and a rising world population, shifting from meat towards more plant-based products holds potential to reduce the environmental impact of our food consumption. Replacing meat in the diet, however, requires compensation through other products that are able to provide the important nutrients present in meat (protein, iron, zinc and vitamin B12). This study applies linear programming techniques with the aim to compose meat replacers, with equivalent nutritional value to meat (using chicken and beef as a reference), minimising the environmental impact with regards to climate change, land use, water use and fossil fuel depletion. A life cycle approach was used to quantify the environmental impacts. Particular attention was given to protein quality and the relative quantities of essential amino acids. The results show that soy is a preferred ingredient due to its favorable amino acid profile. Among the different scenarios investigated, the vegan replacers, with reductions of up to $87 \%$, have the largest potential for impact reduction for all indicators except water use. Insect-based replacers have the largest potential for water use reduction (up to 47\%), but show relatively high fossil fuel depletion values. The smallest improvement potential is observed with regards to fossil fuel depletion, with some values even $45 \%$ higher than the values for meat. Furthermore, it is not possible to obtain equivalent nutritional values to beef without using fortifications. 


\subsection{Introduction and Background}

Food is a vital part of our lives, however current consumption and production patterns pose a threat to the natural environment by contributing to global warming, resource depletion and the extinction of species (Garnett, 2013). The food system as a whole accounts for an estimated 20-30\% of the global greenhouse gas emissions (Vermeulen et al., 2012) while, within food consumption patterns, meat and dairy products are the main contributors to environmental impacts (Notarnicola et al., 2017; Steinfeld et al., 2006). Among the different types of meat, beef has the largest and chicken the smallest environmental impact in terms of climate change, land use and fossil fuel depletion (Hallström et al., 2015). Given a growing population and an increase in wealth the demand for food and meat is predicted to increase by around $70 \%$ by 2050 for food and by 2030 for meat (Fiala, 2008; FAO, 2009). This rise in demand combined with the sustainability aspects faced by the food system, poses major challenges for the future and calls for measures to countervail some of these effects.

Over recent years, scientific literature increasingly focused on the environmental impact of food and dietary consumption patterns. In this context, Hallström et al. (2015) conducted a literature review to assess the impact of dietary change on the environment, comparing 14 articles (published in the period from 2009 to 2014) with a focus on climate change and land use. Furthermore, research has investigated the environmental impact of a number of different dietary scenarios, showing that vegan and vegetarian scenarios are most promising for lowering environmental impacts (Meier and Christen, 2012; van Dooren et al., 2014; Berners-Lee et al., 2012). Thus, replacing meat within food consumption patterns and shifting towards more environmental friendly products could reduce the environmental impact of our food system (Health Council of the Netherlands, 2011).

An often used method to evaluate the environmental impact of food products is Life Cycle Assessment (LCA) (Mogensen et al., 2009) (see Appendix 6.B for a description of the LCA method). Several studies apply LCA to compare the environmental impact of meat products with the environmental impact of meat replacers, based on indicators like climate change, land use, water use, ozone layer depletion, human toxicity, acidification and eutrophication. Using an LCA approach, Smetana et al. (2015) show that the environmental impacts of different meat replacers are lower than the environmental impact of chicken, except for lab-grown and mycoprotein-based meat replacers, due to a high energy demand for medium cultivation. Zhu and van Ierland (2004) show similar results, concluding that a pea-based meat replacer has a lower environmental impact than pork in terms of the studied environmental impact indicators.

However, when replacing meat, it should be ensured that the nutritional requirements of the consumer are still met. Meat is an important source of high quality protein, iron, zinc and vitamin B12 (Bender, 1992). Protein quality is determined by the relative quantities 
of essential amino acids, i.e. amino acids that cannot be synthesized by the human body (Bender, 1992). In particular, protein quality is fully determined by the amino acid that is present in the lowest amount relative to human requirements, the so-called limiting amino acid. The other amino acids cannot compensate for a shortage of the limiting amino acid, so if the limiting amino acid is present in low quantities the nutritional quality of the protein will be seriously affected (FAO/WHO/UNU, 2002). Food consumption patterns should therefore contain every essential amino acid in an amount that meets the corresponding human requirements. Meat proteins are of good quality because they contain all the essential amino acids in a proportion resembling that of the human needs (Asgar et al., 2010).

Most of the important nutrients of meat can also be found in plant-based products. However, the nutrients are present in different proportions and often in different forms (Broekema and Blonk, 2009). Moreover, plant-based products do not contain vitamin B12 (Broekema and Blonk, 2009) and have a less favorable amino acid composition than meat (Asgar et al., 2010). An option to meet the requirements for iron, zinc and vitamin B12 in a meatless food consumption pattern is to combine plant-based products with animal-based products, like eggs and dairy products. Another option is to supplement plant-based products with fortifications (i.e. nutrient supplements) (Broekema and Blonk, 2009).

Combining environmental constraints with nutritional considerations, literature increasingly recognises modeling techniques (such as linear programming) as a tool to optimise dietary intake with regards to different criteria. Examples of such diet models, taking into account nutritional as well as environmental aspects using linear programming, are found in MacDiarmid et al. (2012), Tyszler et al. (2016), Tyszler et al. (2014) and Wilson et al. (2013).

MacDiarmid et al. (2012) and Tyszler et al. (2016) have shown that the elimination of meat and dairy products has the biggest effect on decreasing environmental impacts, but that an entirely vegan diet might prove difficult in terms of acceptance within the population and therefore might not be adopted. Tyszler et al. (2014) concluded that a scenario in which meat is replaced with soy-based products has a lower environmental impact, however an additional serving of fish has to compensate for a lack of limiting amino acids as the replacement product is not nutritionally equivalent to meat. Wilson et al. (2013) show a slightly higher environmental impact for the vegan diet in comparison to other scenarios investigated, although they illustrate that a shift from meat towards more plantbased foods can have a positive effect on cost, health and environmental objectives.

The study of Temme et al. (2013) shows that the environmental impact of the diet can be reduced by replacing meat and dairy products with plant-based products without having a negative impact on the iron intake while even lowering saturated fatty acids. One of the limitations of the study, however, is that the lower absorption rate of heme iron was not 
considered. Davis et al. (2010) investigate the environmental impact of four different meal scenarios with different protein sources. While their findings show that the plant-based alternative protein source (pea-burger) has a lower environmental impact, the study does not elaborate on the protein quality of this alternative. The study of Sonesson et al. (2017), comparing different functional units based on protein content in the context of LCA, however, shows that protein quality and digestibility is of importance and affects the environmental impact per unit.

While research shows that plant-based diets generally have a lower environmental impact, culture and acceptance plays an important role when comparing and adopting these dietary scenarios. Replacing meat by unprocessed products like legumes or pulses changes the component structure of the meal and might therefore impede acceptance, whereas using instant meat substitute can facilitate the replacement of meat due to increased convenience, low cooking skill requirements and maintaining the component structure of the meal (Schösler et al., 2012). From a nutritional perspective it is however important to compare the actual nutritional contribution of such replacement products. While the diet is considered the best food unit to evaluate nutritional intake (Van Kernebeek et al., 2014), we believe there is a benefit of designing replacement products that can provide the equivalence of the important nutrients of meat and at the same time make it easy and convenient for the consumer to switch from meat to a more sustainable product. Thus, the aim of this study is to find the optimal combination of non-meat ingredients to compose meat replacers that have an environmental impact as low as possible and a comparable nutritional value to meat. Replacing meat with these meat replacers could reduce the environmental impact of our food system without compromising the nutritional requirements of the population.

The results of this study are useful for producers of meat replacers that want to reduce environmental impacts and offer products that contain the equivalence of the valuable nutrients present in meat. Consumers that are concerned about the environmental impact of their consumption can integrate these products in their food consumption pattern as a convenient replacement for meat. Furthermore, the results can be useful for governments that want to estimate the potential impact of dietary shift from meat to meat replacers.

\subsection{Methods}

This study uses a linear programming model to find the composition of meat replacers that have an environmental impact as low as possible and provide the equivalence of the important nutrients in meat. 


\subsubsection{Scenarios: Four types of meat replacers}

We modelled four types of meat replacers: vegetarian, vegan, insect-based and fortification-free. The vegetarian meat replacer may contain animal-based ingredients, but no insects. The vegan meat replacer may only contain plant-based ingredients. The insect-based meat replacer has insects as its main protein source. The fortification-free meat replacer may not contain any fortification (i.e. supplements of vitamins or minerals). Beef and chicken are used as references in the model, since they represent the largest and the smallest environmental impact of meat, respectively.

Two kinds of results are presented, for which Figure 6.1 defines the boundaries: ingredient results and end product results. The ingredient results (full line box) are calculated by summing the environmental impacts of the selected ingredients up to the point of processing them into the meat replacer. For some ingredients, this includes a processing step to convert raw materials into ingredients (e.g. from soy beans to soy flour). Other ingredients are used directly in the form they are produced in the agricultural production step (e.g. eggs).

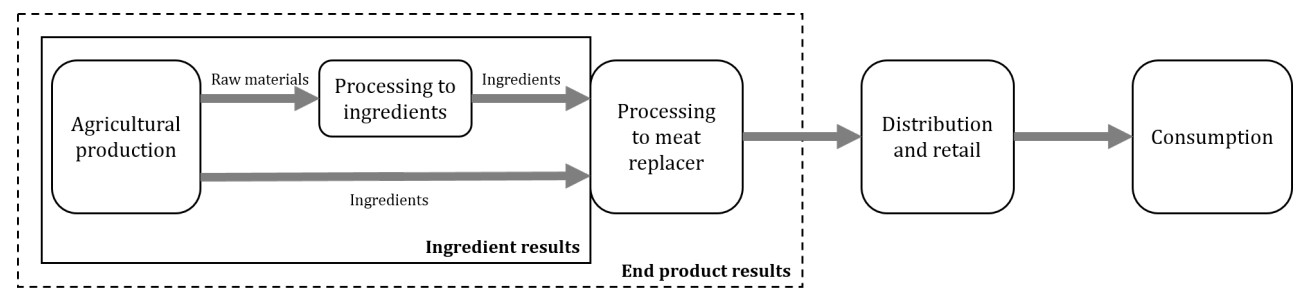

Figure 6.1: System boundaries for the ingredient results (full line box) and end product results (dashed line box), with the grey arrows indicating transportation steps

Ingredient results are converted into end product results (dashed line box) by adding the environmental impact of the processing steps that convert the ingredients into a meat replacer. From consumer perspective, the end products are replacements for the references (raw chicken and raw beef steak), since they all need the same home preparation, e.g. baking.

\subsubsection{Selection of nutrients, environmental impacts and ingredients}

\section{Nutrients}

The model includes the nutrients for which meat is an important source: protein, iron, zinc and vitamin B12. In order to ensure sufficient protein quality, protein is included 
on the level of individual essential amino acids: lysine, leucine, isoleucine, tryptophan, threonine, methionine + cystine, phenylalanine + tyrosine and valine. Iron is expressed in non-heme iron, see Appendix 6.A. The required contents of protein, iron, vitamin B12 and zinc are based on the contents in chicken and beef. The required contents of essential amino acids are based on the human requirements for these amino acids, see Appendix 6.A.

\section{Environmental impacts}

Based on a literature review of LCA studies (see Appendix 6.B), we selected suitable environmental impact indicators. Since this study focuses on environmental impact, indicators that only affect the Area of Protection (AoP) human health $(\mathrm{H})$ are left out. For the AoP natural environment, indicators with the following characteristics are selected:

- frequently used

- influence the global environment

- classified in category 1 of Hauschild et al. (2013)

- related to food production

Climate change is selected as an impact indicator, satisfying all these requirements. In this study, climate change is defined as Global Warming Potential (GWP), expressed in $\mathrm{kg} \mathrm{CO}_{2}$ equivalents. Although eutrophication and acidification are related to food production and are often used, they are not selected because they only affect the local environment and because their assessment method is not of sufficient quality (see Table 6.7). For the AoP natural resources, fossil fuel depletion and water use are selected because of their relation to food processing. Energy use is also often used as indicator, but includes other energy forms than fossil energy sources, like nuclear and renewable energy (Weidema et al., 2013). In this study, the indicator fossil fuel depletion is used, referring to the use of fossil fuels like coal, oil and natural gas (Goedkoop et al., 2013). We made this choice because we want to focus on the depletion of natural resources, which are, in terms of energy, mainly fossil fuels (Goedkoop et al., 2013). Land use does not follow a standardized approach (Smetana et al., 2015), which is demonstrated by the fact that it is classified in the third category in the article of Hauschild et al. (2013). However, it is an important impact category when it comes to feed and food (Smetana et al., 2015) and is used in a large number of LCA studies of food products. That is why land use is selected. In conclusion, four impact indicators are selected: climate change, land use, water use and fossil fuel depletion. 


\section{Ingredients}

The model uses ingredients that are sources of the same nutrients as meat, while having a lower environmental impact. Legumes and wheat are included, because they are good sources of protein, iron and zinc (Broekema and Blonk, 2009; Voedingscentrum, 2016). They are commonly used in the form of flour, protein concentrate or protein isolate (Averink, 2015). Eggs and dairy products are included as they are good sources of high quality protein, iron, zinc and vitamin B12 (Voedingscentrum, 2016), while inducing a lower environmental impact than meat in terms of climate change, land use and fossil fuel depletion (de Vries and de Boer, 2010). Insects have recently gained interest for use as meat replacers. They are generally high in protein, vitamins and minerals (Bukkens, 1997). The production of mealworms results in a lower climate change value and requires less land than the production of meat (Oonincx and De Boer, 2012). Therefore, two sorts of insects, the mealworm (Tenebrio molitor) and the super worm (Zophobas morio), are included in the model. Iron-, zinc- and vitamin B12 fortifications are included to ensure that the requirements for these nutrients can be met. The full list of ingredients, with their nutrient contents and environmental impacts, can be found in Appendix 6.C.

\subsubsection{Data inputs}

\section{Data collection}

The main nutritional data were retrieved from the NEVO database (RIVM, 2013). Data that are not available in this database were retrieved from the online nutrient database of the United States Department of Agriculture (USDA, 2016) and from literature (Broekema and Smale, 2011; Friedman, 1996; Davis et al., 1994; FAO/INFOODS, 2013; Flindall, 2016). Life cycle environmental impacts of the studied products were obtained from various sources as shown in Table 6.1.

When data is used from different LCA studies, the system boundaries, functional unit and allocation method should ideally be the same (de Vries and de Boer, 2010). All the consulted studies use a functional unit of $1 \mathrm{~kg}$ of product and applied economic allocation. As can be seen in Table 6.1, the system boundaries of all the studies start 'from cradle', which means that the environmental impact includes all the upstream life cycles steps, starting at the primary cultivation step. The boundaries are until the farm or factory gate, which indicates that life cycle steps are taken into account up to the point that the products leave the farm or factory (Blonk Agri-footprint BV, 2015a). As presented in Figure 6.1, the environmental impacts of all the ingredients go up to the point of processing into the meat replacer. This corresponds with the farm and factory gate of the consulted studies. The geographical boundaries of all the studies relate to the Netherlands, except 
for the water footprint network. This study did not provide sufficient local data for the Netherlands, therefore a global average is used.

Table 6.1: Consulted studies, their studied products, system- and geographical boundaries as well as the type of environmental impact values obtained from the studies

\begin{tabular}{|c|c|c|c|c|}
\hline Study & $\begin{array}{l}\text { Studied } \\
\text { product }\end{array}$ & System boundary & $\begin{array}{l}\text { Geographical } \\
\text { boundary }\end{array}$ & $\begin{array}{l}\text { Obtained environmental } \\
\text { impact values }\end{array}$ \\
\hline $\begin{array}{l}\text { Blonk et al. } \\
(2007)\end{array}$ & Meat (fresh) & $\begin{array}{l}\text { From cradle to farm } \\
\text { gate }\end{array}$ & The Netherlands & $\begin{array}{l}\text { Climate change, land use, } \\
\text { fossil fuel depletion }\end{array}$ \\
\hline $\begin{array}{l}\text { Broekema and } \\
\text { Smale (2011) }\end{array}$ & Legumes & $\begin{array}{l}\text { From cradle to } \\
\text { factory gate }\end{array}$ & $\begin{array}{l}\text { Processing in the } \\
\text { Netherlands, } \\
\text { cultivation in most } \\
\text { important producing } \\
\text { countries }\end{array}$ & $\begin{array}{l}\text { Climate change, land use, } \\
\text { fossil fuel depletion }\end{array}$ \\
\hline $\begin{array}{l}\text { Mollenhorst } \\
\text { et al. (2006) }\end{array}$ & Eggs & $\begin{array}{l}\text { Cradle to farm gate, } \\
\text { average of four egg } \\
\text { production systems }\end{array}$ & The Netherlands & $\begin{array}{l}\text { Climate change, land use, } \\
\text { fossil fuel depletion }\end{array}$ \\
\hline $\begin{array}{l}\text { Van Middelaar } \\
\text { et al. (2011) }\end{array}$ & Cheese & $\begin{array}{l}\text { Cradle to factory } \\
\text { gate }\end{array}$ & The Netherlands & $\begin{array}{l}\text { Climate change, land use, } \\
\text { fossil fuel depletion }\end{array}$ \\
\hline $\begin{array}{l}\text { Oonincx and } \\
\text { De Boer (2012) }\end{array}$ & Meal worms & Cradle to farm gate & The Netherlands & $\begin{array}{l}\text { Climate change, land use, } \\
\text { fossil fuel depletion }\end{array}$ \\
\hline $\begin{array}{l}\text { Mekonnen and } \\
\text { Hoekstra (2011) }\end{array}$ & Legumes & $\begin{array}{l}\text { Cradle to farm/ } \\
\text { factory gate }\end{array}$ & Global & Water use \\
\hline $\begin{array}{l}\text { Mekonnen and } \\
\text { Hoekstra (2013) }\end{array}$ & $\begin{array}{l}\text { Meat, eggs, } \\
\text { cheese, meal } \\
\text { worms }\end{array}$ & $\begin{array}{l}\text { Cradle to farm/ } \\
\text { factory gate }\end{array}$ & Global & Water use \\
\hline
\end{tabular}

Data concerning applied processes to convert the ingredients into meat replacers were obtained from a producer of meat replacers (Averink, 2015). The data comprise the amount of electricity, natural gas and water used during processing. The climate change value of the processing step is calculated by considering the amount of natural gas that is combusted during processing (Aubé, 2001) and the amount of electricity that is used. To calculate the amount of greenhouse gas emission, the amount of combusted natural gas at the processing factory are added to the amount of fossil fuels combusted at the electricity power plant (approximately $78 \%$ of all electricity sources in the Netherlands (Wernet et al., 2016)) and multiplied with the amount of greenhouse gas emission that occur by burning fossil fuels. Since the agricultural production of food products is the main contributor in terms of land use (Broekema and Blonk, 2009), the processing step is expected to not contribute considerably to the land use value of the meat replacers. Therefore, this impact indicator is not included in the end product results. The water 
use value is calculated by considering the amount of water required to produce $1 \mathrm{~kg}$ of meat replacer. The fossil fuel depletion value of the processing step was calculated by considering the amount of natural gas and electricity used. As in the calculation of the climate change value, it is assumed that $78 \%$ of the electricity sources comprise fossil fuels (Wernet et al., 2016).

The nutritional and environmental data of all the ingredients and the references can be found in Appendix 6.C, as well as the environmental data of the references.

\section{Assumptions about data}

This study assumes that the processing of ingredients into meat replacers takes place in the Netherlands. The environmental data of the ingredients and the references include the impact of agricultural production of the products in a certain country, the transport to the Netherlands and for some ingredients the conversion into specific ingredients. The origins of the included legumes are based on the most important countries that produce the legumes for processing in the Netherlands (Broekema and Smale, 2011). The model can be applied to other countries by inserting corresponding data.

It is assumed that all the used legumes exist in the form of flour, protein concentrate and protein isolate and are used as ingredients in these forms. In reality, not all legumes may exist in these forms (Broekema and Smale, 2011). Whereas nutritional data of all the included legumes in dry form are available, nutritional data of flour, protein concentrate and protein isolate are only available for soy. The nutritional value of the flours of the other legumes is assumed to be equal to that of dry legumes. The nutritional value of their protein concentrates and isolates are estimated using conversion factors that exist between the nutritional value of dry soy beans, soy protein concentrate and soy protein isolate. For chickpeas, kidney beans, lentils and wheat, only environmental data of their unprocessed form is available. For soy, peas and lupine, environmental data about flour and concentrate is available and for soy and peas environmental data about the protein isolate form is available. Based on soy, estimations of environmental data of flour, concentrate and isolate of chickpeas, kidney beans, lentils and wheat are made.

Environmental data about fortifications are not available. They are generally assumed to have a relatively high environmental impact, since it requires a lot of purification steps to obtain them (Broekema and Smale, 2011). However, only a very small amount of fortification has to be added to achieve the required amount of the nutrient, so it is assumed that their contribution is not very high. That is why an estimation of the environmental impact values of the fortifications is made. For every environmental impact indicator, the largest value of all ingredients is taken and rounded up. These values are chosen as the environmental impact values of the fortifications. In this way, it is assumed that the fortifications have higher environmental impact values than all the other ingredients. To 
study the effect of changes in the environmental impact values of the fortifications, we carried out a sensitivity analysis on these values (Appendix 6.E).

We only consider the nutritional composition of the meat replacer and did not consider the nutritional composition of the complete diet. The absorbability of zinc and non-heme iron are however dependent on the presence of certain components in the diet, which influences the required content of zinc and non-heme iron in the meat replacers. To investigate the effect of changes in the required contents, a sensitivity analysis is executed (Appendix 6.E).

Based on the electricity, natural gas and water consumption from practice (Averink, 2015), the climate change, water use and fossil fuel depletion values for the meat replacers during processing are estimated. Data for specific processes or products are not available (Averink, 2015). Therefore, the environmental impact values of the processing phase are estimated from the total amount of electricity, natural gas and water consumed and by dividing these values by the total amount of produced products. Consequently, the environmental impact values during processing is assumed to be constant and not dependent on the ingredients that are used. 


\subsubsection{Mathematical model}

The model uses the following indices, variables, and parameters:

Table 6.2: Summary of notation

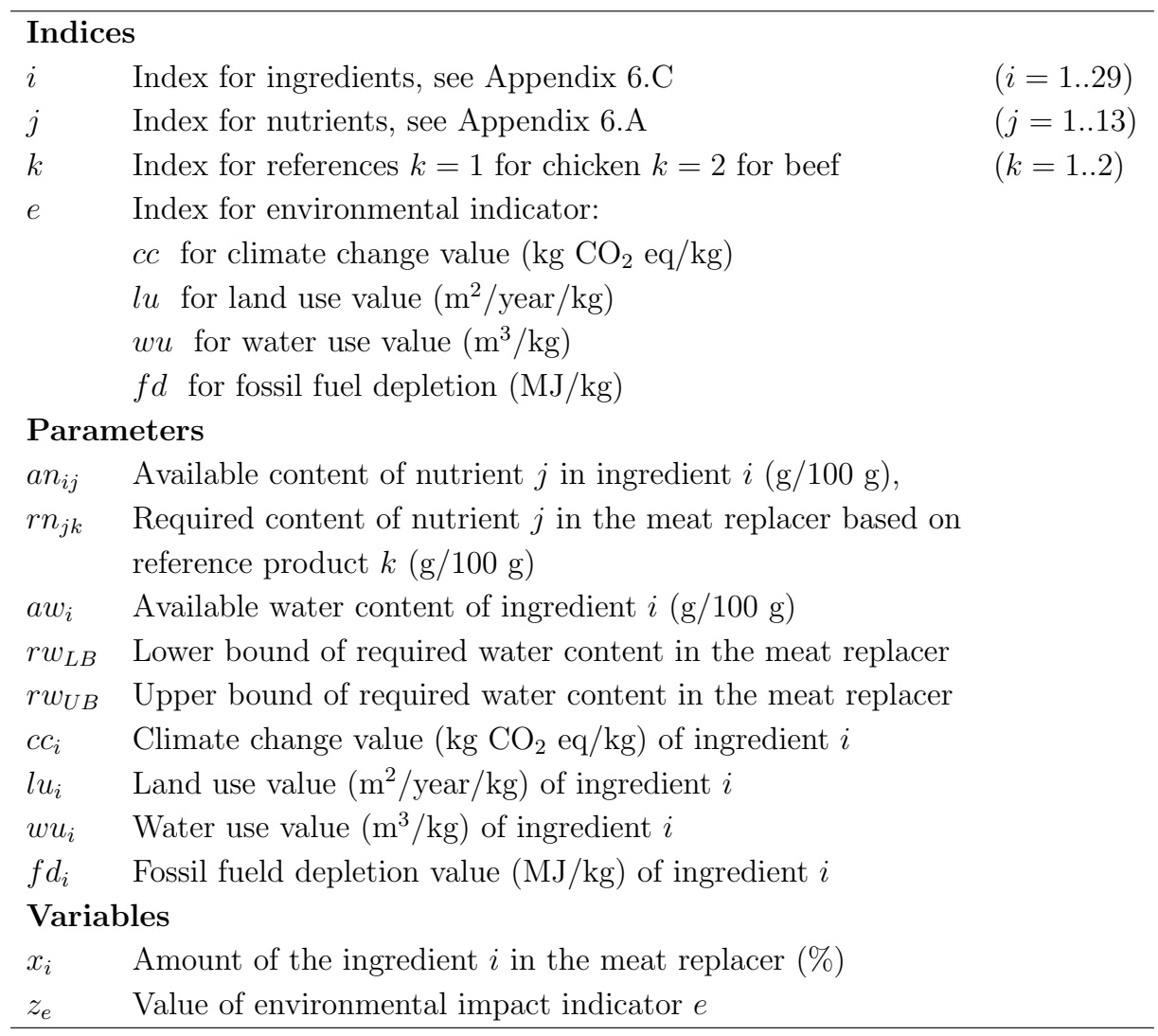

The model contains four types of constraints: expressions for calculating environmental impact indicators, nutritional constraints, technical constraints, scenario constraints.

\section{Environmental impact indicators}

The environmental impact indicators of the meat replacers are calculated from the contributions of the ingredients: 
$Z_{c c}=\sum_{i=1}^{29} c c_{i} x_{i}$

$Z_{l u}=\sum_{i=1}^{29} l u_{i} x_{i}$

$Z_{w u}=\sum_{i=1}^{29} w u_{i} x_{i}$

$Z_{f d}=\sum_{i=1}^{29} f d_{i} x_{i}$

\section{Nutritional Constraints}

Nutritional constraints are based on the nutritional value of the references chicken $(k=1)$ and beef $(k=2)$. With $\mathrm{k}$ as a reference, the amount of nutrient $j$ contributed by the ingredients $i$ should at least be equal to the amount of nutrient $j$ in that reference $\left(r n_{j k}\right)$ :

$$
\sum_{i=1}^{29} a n_{i j} x_{i} \geq r n_{j k} \quad j \in\{1 . .13\}
$$

\section{Technical Constraints}

The total weight of the meat replacer should be $100 \mathrm{~g}$ (Vivera, 2016):

$$
\sum_{i=1}^{29} x_{i}=100
$$

Since the meat replacer should be a cohesive, consumable end product, the total water content should be between the lower bound $r w_{L B}(55 \mathrm{~g}$ per $100 \mathrm{~g})$ and the upper bound $r w_{U B}(65$ g per $100 \mathrm{~g})$ (RIVM, 2013):

$$
r w_{L B} \leq \sum_{i=1}^{29} a w_{i} x_{i} \geq r n_{j k} \leq r w_{U B}
$$




\section{Scenario Constraints}

The four different scenarios (vegetarian, vegan, insect-based, fortification-free) are modelled by adding one of the following four scenario constraints.

- For the vegetarian meat replacer, no insects are allowed. Therefore, the amounts of meal worms $\left(x_{24}\right)$ and super worms $\left(x_{25}\right)$ should be zero:

$$
x_{i}=0 \quad i \in\{24,25\}
$$

- In the vegan meat replacer, no animal-based product and no insects are allowed. Therefore, the amounts of eggs $\left(x_{22}\right)$, cheese $\left(x_{23}\right)$, meal worms $\left(x_{24}\right)$ and super worms $\left(x_{25}\right)$ should be zero:

$$
x_{i}=0 \quad i \in\{22,23,24,25\}
$$

- The main ingredient of the insect-based meat replacer should be insects. Therefore, the summed contents of meal worms $\left(x_{24}\right)$ and super worms $\left(x_{25}\right)$ should be at least $15 \%$ :

$$
x_{24}+x_{25} \geq 0.15 \quad i \in\{24,25\}
$$

- In the fortification-free meat replacer, no iron- $\left(x_{27}\right)$, vitamin B12- $\left(x_{28}\right)$, or zinc $\left(x_{29}\right)$ fortifications are allowed:

$$
x_{i}=0 \quad i \in\{27,28,29\}
$$

\subsubsection{Approach to multi-objective problem}

Finding the meat replacer with the lowest environmental impact is a multi-objective problem since it requires minimising four conflicting environmental impact indicators $Z_{e}$ at the same time: climate change, land use, water use and fossil fuel depletion. These indicators have different units and scales, so they cannot simply be summed and minimised together. In order to explore the problem, first the indicators $Z_{e}$ with $(e=c c, l u, w u, f d)$ are minimised separately. These minimisations provide the lowest (i.e. best) possible value of each indicator $Z_{e}$, which is referred to as $Z_{e}^{\star}$. The $Z_{e}$ values resulting from the four individual minimisations are put in so-called payoff matrices. In the (fictitious) payoff matrix of Table 6.3, minimising environmental impact indicator $Z_{l u}$ leads to a solution in which $Z_{c c}=0.7, Z_{l u}=3, Z_{w u}=0.04$, and $Z_{f d}=19$. When minimising $Z_{c c}$ to $Z_{f d}$ individually, the obtained values of $Z_{l u}$ range from 3 (when $Z_{l u}$ is minimised) to 8 (when $Z_{f d}$ is minimised). The highest (i.e. worst) value of $Z_{e}$ in the payoff matrix is indicated as 
$\underline{Z}_{e}$, so $Z_{l u}^{\star}=3$ and $\underline{Z}_{l u}=8$. In the payoff matrix the lowest (best) value of $Z_{e}$ is indicated with " $\star$ " and the highest (worst) value is underlined.

Table 6.3: Fictitious example of a payoff matrix and the resulting normalised impact values $d_{e}$

\begin{tabular}{llllllllllll}
\hline & \multicolumn{3}{c}{ Payoff matrix } & \multicolumn{4}{c}{$\begin{array}{c}\text { Normalisation } \\
\text { Observed indicator }\end{array}$} & \multicolumn{3}{c}{ Normalised indicator } \\
\hline \multirow{3}{*}{ Minimised indicator } & $Z_{c c}$ & $0.5^{\star}$ & 5 & $Z_{l u}$ & $Z_{w u}$ & $Z_{f d}$ & & $d_{c c}$ & $d_{l u}$ & $d_{w u}$ & $d_{f d}$ \\
& $Z_{l u}$ & 0.7 & $3^{\star}$ & 0.07 & $\underline{20}$ & & $0^{\star}$ & 0.4 & $\underline{1}$ & $\underline{1}$ \\
& $Z_{w u}$ & $\underline{1.5}$ & 7 & $0.01^{\star}$ & 11 & & 0.2 & $0^{\star}$ & 0.5 & 0.9 \\
& $Z_{f d}$ & 1.2 & $\underline{8}$ & 0.04 & $10^{\star}$ & 0.7 & $\underline{1}$ & 0.5 & $0^{\star}$ \\
\hline
\end{tabular}

Because the four environmental impact indicators have different units and values, the $Z_{e}$ are normalised to values $d_{e}$ in a $0-1$ interval (Romero and Rehmann, 2003), in which 0 represents the lowest value and 1 represents the highest value:

$$
d_{e}=\frac{Z_{e}-Z_{e}^{\star}}{\underline{Z}_{e}-Z_{e}^{\star}} \quad e \in\{c c, l u, w u, f d\}
$$

The right-hand side of 6.3 shows the normalised environmental impact indicators $d_{e}$. For example, $Z_{l u}=5$ (in the first row of the left-hand side) is transformed to $d_{l u}=\frac{Z_{l u}-Z_{l u}^{\star}}{\underline{Z}_{l u}-Z_{l u}^{\star}}=$ $\frac{5-3}{8-3}=0.4$.

Then, the four environmental impact indicators are minimised together by minimising the $d_{e}$ in two ways:

- minsum objective: minimise : $\left\{d_{c c}+d_{l u}+d_{w u}+d_{f d}\right\}$

- $\operatorname{minmax}$ objective: minimise : $\left[\operatorname{maximum}\left\{d_{c c} ; d_{l u} ; d_{w u} ; d_{f d}\right\}\right]$

The minsum objective generates the solution (i.e. the composition of the meat replacer) in which the sum of the normalised impact indicators $d_{e}$ is as low as possible. It is, however, possible that the minsum solution is very unbalanced, i.e. the values of the individual normalised impact indicators are very different (for example: one value is very large and others are very small). In contrast, the minmax objective generates the solution in which the largest among the normalised impact indicators is as small as possible, thus providing a solution that is as balanced as possible (Romero, 2001). 


\subsection{Results}

The model was used to compose vegetarian, vegan, insect-based and fortification-free replacers for chicken and beef. This section provides the ingredient results and end product results (as illustrated in Figure 6.1) of the composed chicken replacers and beef replacers. Their environmental impact values are compared with those of chicken and beef.

\subsubsection{Ingredient results}

\section{Ranges of the environmental impact indicators}

Minimising the four individual environmental impact indicators $Z_{e}$ for the four types of meat replacers yields the payoff matrices (see Appendix 6.D) from which the ranges of the individual impact indicators are observed (see Table 6.4). The payoff matrices for the vegetarian and the vegan chicken replacer are identical, because the optimal solutions are the same. This also is the case for the vegetarian and vegan beef replacer. It is not possible to compose a fortification-free beef replacer: the problem is infeasible when no fortifications are allowed.

Table 6.4: Observed ranges of the environmental impact indicators $Z_{e}$ for the feasible meat replacers

\begin{tabular}{llllllllllll}
\hline \multicolumn{1}{c}{ Chicken replacers } & \multicolumn{4}{c}{ Beef replacers } \\
\hline \multicolumn{1}{c}{ Vegetarian/Vegan } & \multicolumn{1}{c}{ Insect-based } & \multicolumn{1}{c}{ Fortification-free } & \multicolumn{2}{c}{ Vegetarian/Vegan } & \multicolumn{2}{l}{ Insect-based } \\
& $Z_{e}{ }^{*}$ & $\underline{Z_{e}}$ & $Z_{e}{ }^{*}$ & $\underline{Z_{e}}$ & $Z_{e}{ }^{*}$ & $\underline{Z_{e}}$ & $Z_{e}{ }^{*}$ & $\underline{Z_{e}}$ & $Z_{e}^{*}$ & $\underline{Z_{e}}$ \\
\hline$e=c c$ & 0.62 & 1.35 & 0.95 & 2.71 & 1.41 & 2.71 & 0.59 & 1.31 & 0.91 & 2.7 \\
$e=l u$ & 2.64 & 6.51 & 2.84 & 4.81 & 3.43 & 4.81 & 2.52 & 6.32 & 2.69 & 4.65 \\
$e=w u$ & 0.017 & 0.069 & 0.007 & 0.068 & 0.007 & 0.115 & 0.019 & 0.07 & 0.007 & 0.069 \\
$e=f d$ & 7.28 & 15.78 & 11.14 & 34.1 & 8.96 & 34.1 & 6.78 & 15.27 & 10.63 & 34.05 \\
\hline$Z_{e}^{*}$ indicates the best (lowest) values of each indicator, $Z_{e}$ indicates the worst (highest) values of each indicator.
\end{tabular}

Results of minsum and minmax optimisations

Figure 6.2 and 6.3 show the optimal compositions $x_{i}$ of the chicken and beef meat replacers, respectively, for the minsum and minmax objectives. The meat replacers consist mainly of water and soy protein concentrate. The water is needed to ensure that the water content is between 55 and $65 \%$. Soy appears to be a suitable replacer for meat, since it is selected to be the main ingredient for all meat replacers. This is assumingly because of its protein quality, which is comparable with that of meat (Asgar et al., 2010). 

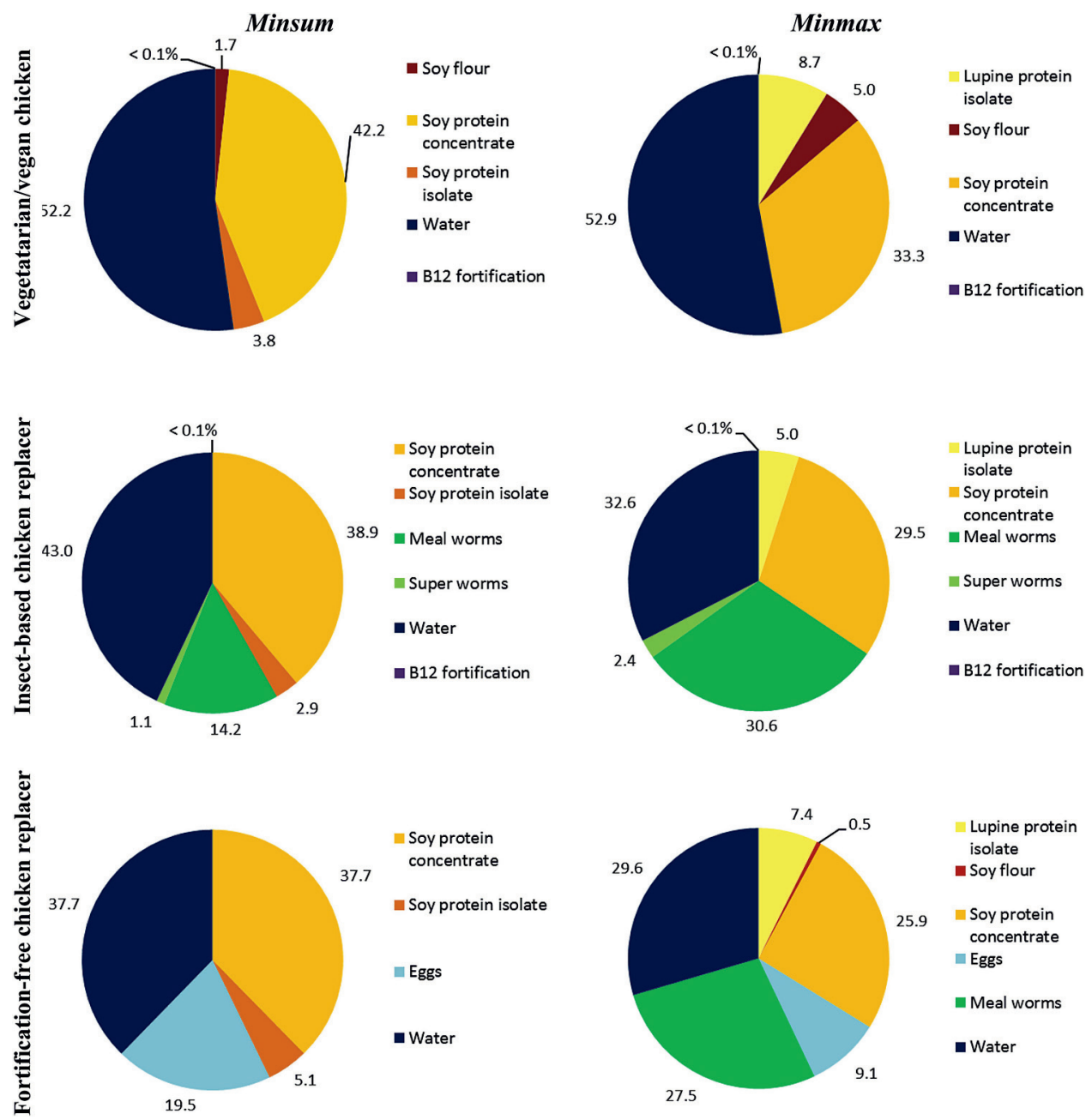

Figure 6.2: Composition of the vegetarian/vegan, insect-based and fortification-free chicken replacers for minsum (left) and minmax (right)

* Vitamin B12 fortification is present in very low quantities; amounts are specified in Appendix 6.F

The vegetarian meat replacers and the insect-based meat replacers contain no animalbased products, although they are allowed. Based on the modelled results, it is more favorable to add fortifications than animal-based products to achieve the required amount of iron, vitamin B12 and zinc. The chicken replacers contain vitamin B12 fortification, but no fortifications for iron and zinc: sufficient amounts of these nutrients are provided through the other ingredients. In the beef replacers, iron-, vitamin B12- and zinc fortifications are necessary to meet the corresponding required amounts. In the fortification-free 
chicken replacer, eggs are used to obtain the required amount of vitamin B12.
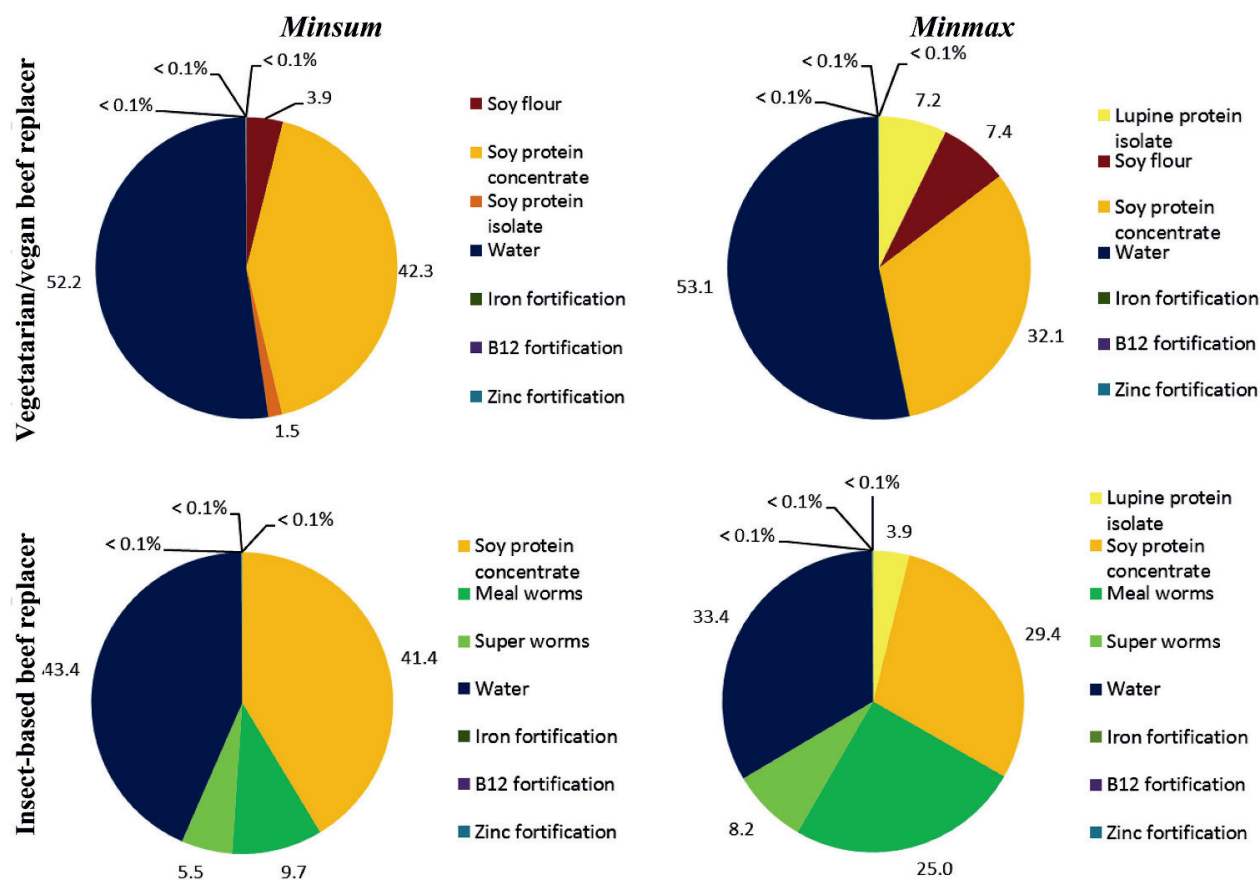

Figure 6.3: Composition of the vegetarian/vegan and insect-based beef replacers for minsum (left) and minmax (right)

* Vitamin B12 fortification is present in very low quantities; amounts are specified in Appendix 6.F

The insect-based meat replacers have to contain at least $15 \%$ meal worms and/or super worms. The meal worms are preferred over the super worms because they have a slightly better nutritional value. For the minsum objectives, the amounts of insects are almost at their minimum allowed level because of their high fossil fuel depletion values. For the minmax objectives, the insect amounts are remarkably higher because of their low water use values, which are minimised in this objective. The fortification-free chicken replacer also contains a considerable amount of meal worms for the minmax objective, although they are not mandatory in this meat replacer. They are preferred over eggs, because their water use is much smaller while they also contain vitamin B12.

Table 6.5 shows the values of the environmental impact indicators $Z_{e}$, and the normalised environmental impact indicators $d_{e}$ for all meat replacers. In every minsum solution, water use $\left(d_{w u}\right)$ has the highest value among the $d_{e}$, and climate change $\left(d_{c c}\right)$ and land use $\left(d_{l u}\right)$ have very low values. The minsum solutions are thus relatively unbalanced. Minimising the maximum among the $d_{e}$ decreases $d_{w u}$ at the cost of increasing $d_{c c}+d_{l u}+d_{f d}$. As a result, the minmax solutions are more balanced than the minsum solutions. 
Table 6.5: Environmental impact indicators $Z_{e}$ and normalised environmental impact indicators $d_{e}$ for all feasible meat replacers

\begin{tabular}{cllllllllll}
\hline \multicolumn{1}{c}{ Chicken replacers } & \multicolumn{3}{c}{ Beef replacers } \\
\hline & \multicolumn{1}{c}{ Vegetarian/Vegan } & \multicolumn{2}{c}{ Insect-based } & \multicolumn{2}{c}{ Fortification-free } & \multicolumn{2}{c}{ Vegetarian/Vegan } & \multicolumn{2}{c}{ Insect-based } \\
& minsum & minmax & minsum & minmax & minsum & minmax & minsum & minmax & minsum & minmax \\
\hline$Z_{c c}$ & 0.62 & 0.73 & 0.95 & 1.39 & 1.42 & 1.68 & 0.59 & 0.7 & 0.91 & 1.37 \\
$Z_{l u}$ & 2.64 & 3.32 & 2.84 & 3.48 & 3.43 & 3.96 & 2.53 & 3.15 & 2.7 & 3.33 \\
$Z_{w u}$ & 0.04 & 0.034 & 0.035 & 0.027 & 0.083 & 0.047 & 0.04 & 0.035 & 0.036 & 0.027 \\
$Z_{f d}$ & 9.32 & 10.06 & 13.37 & 18.55 & 11.36 & 18.25 & 8.81 & 9.52 & 12.93 & 18.25 \\
\hline$d_{c c}$ & 0.01 & 0.15 & 0 & 0.25 & 0 & 0.21 & 0 & 0.15 & 0 & 0.26 \\
$d_{l u}$ & 0 & 0.18 & 0 & 0.32 & 0 & 0.37 & 0 & 0.17 & 0 & 0.33 \\
$d_{w u}$ & 0.44 & 0.33 & 0.46 & 0.32 & 0.7 & 0.37 & 0.42 & 0.32 & 0.46 & 0.33 \\
$d_{f d}$ & 0.24 & 0.33 & 0.1 & 0.32 & 0.1 & 0.37 & 0.24 & 0.32 & 0.1 & 0.33 \\
\hline
\end{tabular}

Figure 6.4 presents the environmental impact values of the meat replacers, and the contributions of their ingredients. They are compared with the environmental impact values of chicken and beef. The grey pictograms above the bars indicate which reference is used. Note, that the climate change value of beef $(7.53 \mathrm{~kg} \mathrm{CO}$ eq $/ \mathrm{kg})$ does not fit on the scale. The figure shows, that for climate change, land use and fossil fuel depletion, the vegetarian/vegan meat replacers have the lowest impact for both minimisations and for both references. For water use, the insect-based meat replacers have the lowest impact for both minimisations and references. For climate change and land use, the meat replacers have lower impact values than their references. For water use, the vegetarian/vegan and insect-based meat replacers have lower impact values than their references. Only the fortification-free chicken replacer in the minsum optimization has higher water use value than chicken, which is mainly due to the high water use value of eggs. In the minmax optimization, however, the water use of the fortification-free chicken replacer is lower than that of chicken, because of the low water use of meal worms. For fossil fuel depletion, the values of all meat replacers in the minsum optimisation are lower than that of their references.

In the minmax optimisation, the fossil fuel depletion values of the insect-based and fortification-free chicken replacers are slightly higher than those of chicken, because of the high contribution of meal worms. Soy protein concentrate contributes greatly to all environmental impact values because of its high content in all meat replacers. The overall contributions of the iron-, vitamin B12- and zinc-fortifications are very low, because only small amounts of them are needed to achieve the required amount of these nutrients. The environmental impact values for beef replacers are smaller than those for chicken replacers, because the protein content of beef is slightly lower than that of chicken. As the required protein amount is a binding constraint it has a direct effect on the solution. 

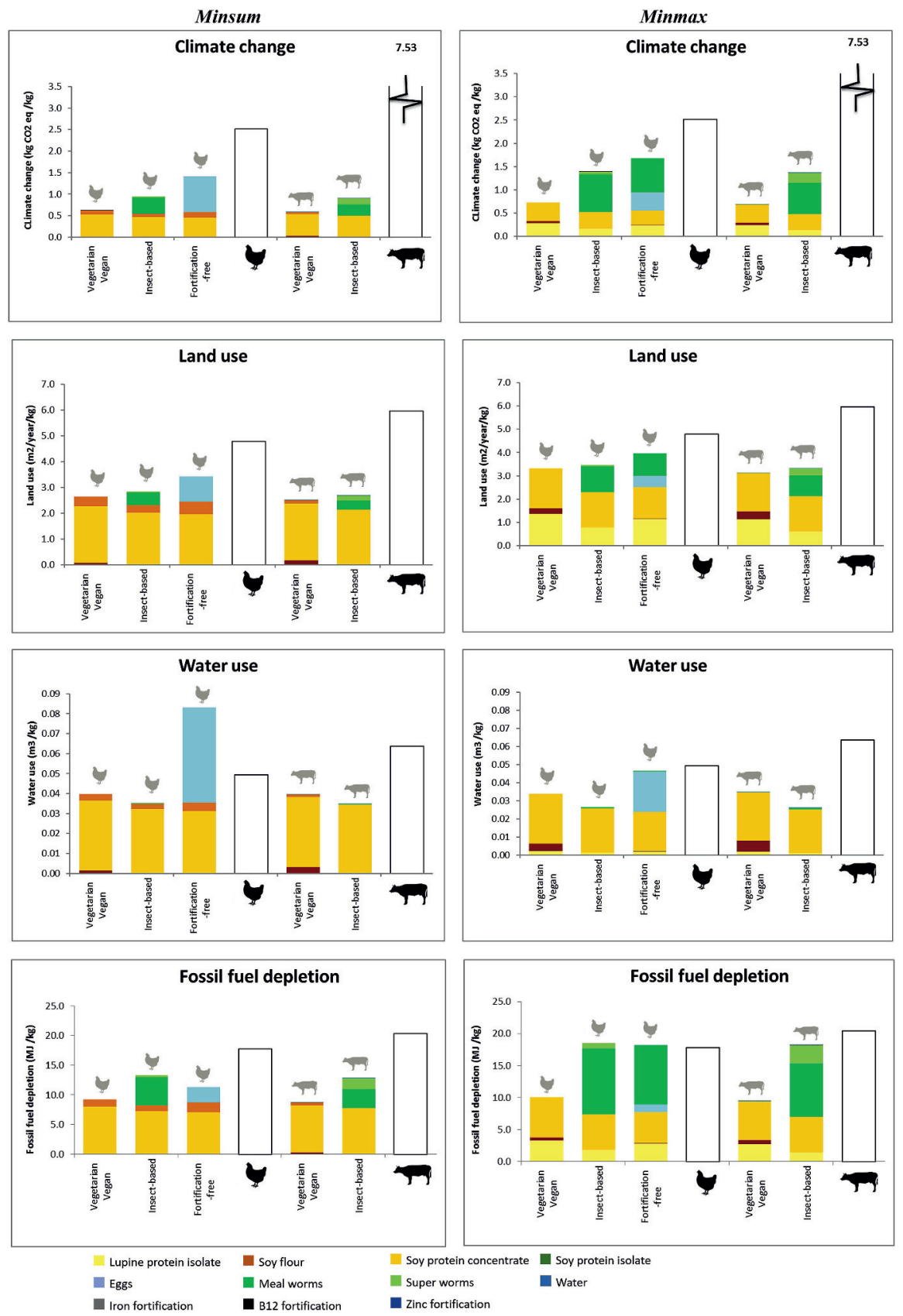

Figure 6.4: Environmental impact values of the meat replacers, specified per ingredient, compared with the environmental impact values of chicken and beef, for the minsum (left) and the minmax optimisation (right). 


\subsubsection{End product results}

Figure 6.5 visualizes the total climate change, water use and fossil fuel depletion values of the meat replacers as end products. The grey pictograms above the bars indicate again which reference is used. The contribution of the ingredients (as given in the ingredient results) and the processing step (highlighted in grey) are represented separately.
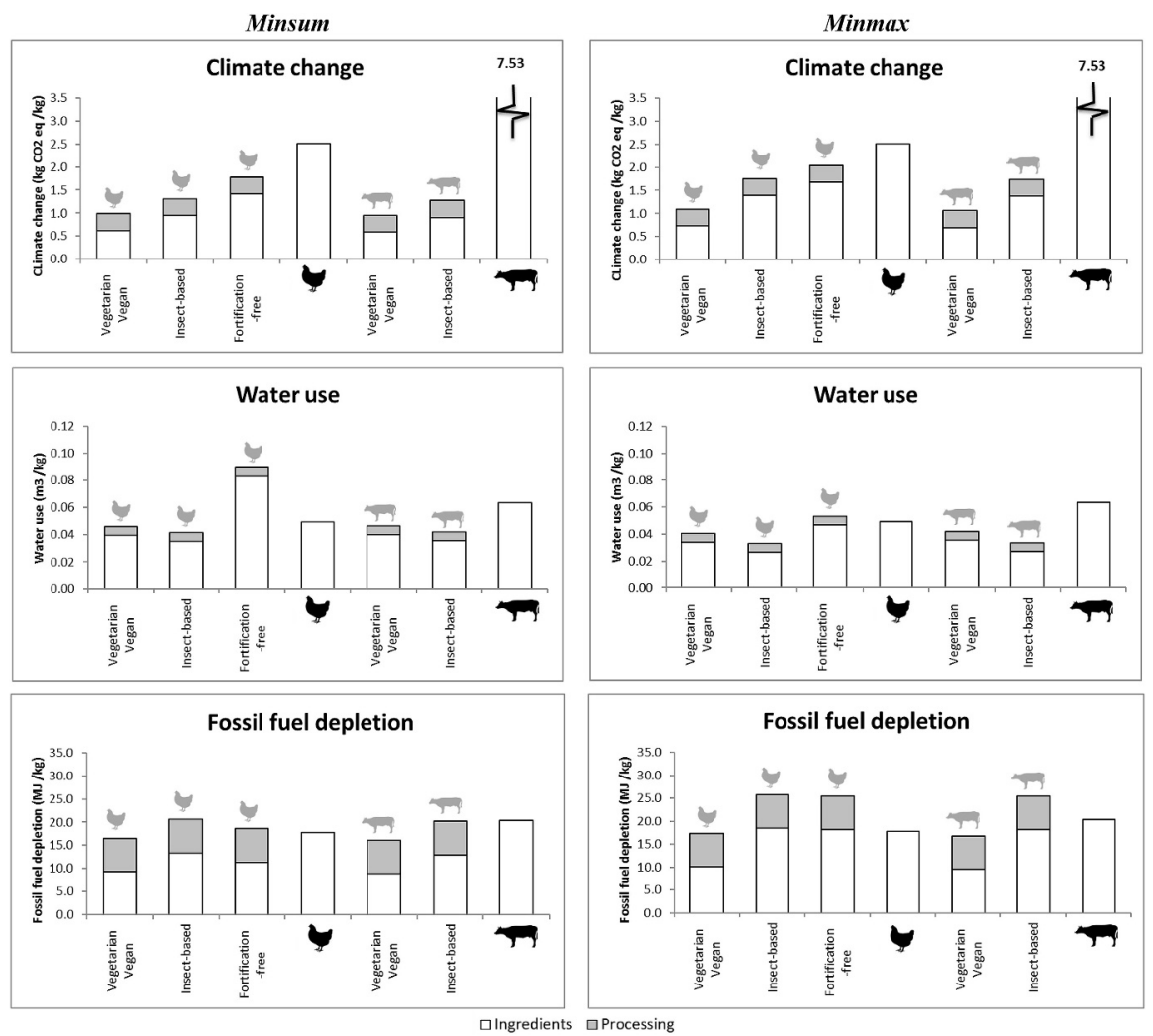

Figure 6.5: End product results: climate change, water use and fossil fuel depletion of the meat replacers compared with the environmental impact values of chicken and beef, for the minsum optimization (left) and minmax optimization (right)

For climate change, the processing phase has a considerable contribution compared to the contribution of the ingredients: on average $26 \%$ for the chicken replacers and $30 \%$ for the beef replacers. However, as in the ingredient results, the climate change values of the replacers are all smaller than the climate change value of their reference. For water use, the processing phase has a relatively low contribution: on average $14 \%$ of the total water use value for the chicken replacers and $16 \%$ for the beef replacers. As in the 
ingredient results, the water use of the fortification-free chicken replacer exceeds the water use of chicken in the minsum solutions. In the minmax solutions, the contribution of the processing step makes the water use of the fortification-free chicken replacer exceed the water use of chicken. The water use values of the beef replacers are still lower than the value of beef for both optimizations, as in the ingredient results. For fossil fuel depletion, the processing step contributes on average $36 \%$ for the chicken replacers and $38 \%$ for the beef replacers. By adding the processing step, the fossil fuel depletion value of the insect-based and fortification-free chicken replacer for the minsum optimization exceed the value of chicken. As in the ingredient results, the fossil fuel depletion of the insectbased and fortification-free chicken replacers for the minmax optimization exceed the value of chicken. Also the fossil fuel depletion value of the insect-based beef replacer for the minmax optimization exceeds the value of beef by adding the processing step.

\subsection{Discussion}

The need for more sustainable food choices is obvious, as current consumption and production patterns threaten the environment. Replacing meat, as one of the main contributors to these threats, can lower the burden and has been investigated by a variety of researchers (Hallström et al., 2015; MacDiarmid et al., 2012; Wilson et al., 2013; Tyszler et al., 2014; Temme et al., 2013). However, meat also provides important nutrients in the diet and thus needs to be substituted by other products that can compensate for the reduction in meat. The results of this study show that it is possible to compose meat replacers with a lower environmental impact and an equivalent nutritional contribution with regards to energy, iron, zinc, vitamin B12 and the essential amino acids. One of the main advantages of such a meat replacer is that it can be used as a direct substitute and does not require further dietary changes, facilitating the shift to more sustainable food consumption (Schösler et al., 2012). Previous research has focused more on the comparison of different dietary scenarios (Meier and Christen, 2012; Berners-Lee et al., 2012; van Dooren et al., 2014), the design of alternative diets and meals (MacDiarmid et al., 2012; Wilson et al., 2013; Tyszler et al., 2016) or the replacement of individual products within such scenarios (Tyszler et al., 2014; Temme et al., 2013; Davis et al., 2010). The diet is generally viewed as the best unit to evaluate nutritional choice and adequacy (Van Kernebeek et al., 2014). However, former research showed that a reduction of meat often leads to significant changes within the diet (Schösler et al., 2012). This could be avoided by designing products that are equivalent and therefore instant replacements.

Given the list of ingredients, nutritional equivalence was feasible for all scenarios with the exception of a fortification free beef replacer, due to the amount of iron, vitamin B12 and zinc present in beef. For the feasible scenarios, the results of this study indicate that the 
vegan replacements have the largest potential to reduce environmental impact in terms of climate change and land use, with reduction of up to $87 \%$ and $58 \%$, respectively. This is consistent with previous studies investigating different dietary scenarios (Hallström et al., 2015; van Dooren et al., 2014) and studies comparing meat with meat replacers (Smetana et al., 2015; Zhu and van Ierland, 2004). Adding to previous work, this study further concluded that this also holds for fossil fuel depletion (with reductions up to $21 \%$ ), while with regards to water use the best results are obtained for the insect-based replacer (with reductions up to $47 \%$ ), due to the small water requirements of super worms and meal worms. The results show that it is not always favorable to replace chicken and/or beef due to high fossil fuel depletion values of the replacements. For the insect-based chicken replacer for example, the fossil fuel depletion value is $45 \%$ higher than the value of chicken (for the minmax optimization). This can be mainly attributed to the fact that the difference between the replacers and their references in the ingredient results is already small and the processing step adds considerably to the fossil fuel value. From the results we can see a shifting of the burden between environmental impact categories depending on the ingredients used. Minimising the environmental impact of one category can thus lead to negative effects with respect to another, as trade-offs exist between the different environmental impact categories. This also applies to the shift from meat to plant-based alternatives when incorporating the processing step in the results. Further investigation of the processing step could help to gain insights into potential areas of improvement, making this step more efficient and environmentally friendly. In this context however, it should also be noted that the replacers are already preprocessed and thus might require less energy and preparation than meat at the household stage, where energy use is often less efficient (Sonesson et al., 2005). Future research could try to incorporate environmental impacts of the household stage to provide a more comprehensive analysis.

With regards to the ingredients, the findings of this research identify soy as a key ingredient when trying to mimic the nutrient profile of meat, due to its amino acid contribution and its low environmental impact. More recently, the environmental impact of soy has, however, come under scrutiny and been subject to debate and substantial criticism within the research community due to its link to climate change and deforestation (Costa et al., 2007; Arima et al., 2011). Research shows that linkages exist between the production of soy and deforestation levels in major forest regions within Brazil and Argentina, such as the Amazon rainforest and the Argentinian Dry Chaco (Lima et al., 2011; Gasparri et al., 2013). This can have major implications for the environmental impact of soy and indirectly also of meat, with soy being one of the main feed ingredients in today's livestock systems and most of the soy production being triggered by the demand for feed (Billen et al., 2014; Viglizzo et al., 2011). It should thus be noted that there are concerns regarding the environmental impact of soy, with significant differences between production systems and/or regions (Da Silva et al., 2010; Castanheira and Freire, 2013). However, as the 
conversion of plant-based to animal protein is inefficient (Aiking, 2011), a shift from meat to soy-based products, provided this leads to a lower consumption of meat, is unlikely to add further pressure (see Appendix 6.G for a more extensive overview/discussion).

While this study provides valuable insights, adding to the scientific research on dietary intake and environmental impacts, it should be noted that there are some underlying assumptions and limitations that require further attention. One of the main limitations of this study, is the assumption that the ingredients of the model are combined and processed into a meat replacer without taking into account structure and taste. In practice, functional ingredients have to be added to obtain a good structure and taste (Averink, 2015). These ingredients could increase the environmental impact of the meat replacer substantially: the functional ingredient chicken egg protein, for example, has very high values for climate change $(16.59 \mathrm{~kg} \mathrm{CO}$ eq/ $\mathrm{kg})$, land use $\left(31.02 \mathrm{~m}^{2} / \mathrm{y} / \mathrm{kg}\right)$ and fossil fuel depletion (13.04 MJ/kg) (Broekema and Blonk, 2009). To get more information about the use of functional ingredients and their environmental impact, more research in collaboration with producers of meat replacers could be of interest.

Furthermore, with regards to nutritional equivalency, this study only considers the most important nutrients present in meat. Therefore, not taking into account additional nutrients provided by the plant-based ingredients or other factors that could for instance affect absorption rates. The absorbabilities of non-heme iron and zinc, for example, depend on the presence of specific components in the diet. Phytates, for example, inhibit the absorption of zinc and non-heme iron, which means that more of these nutrients should be consumed when phytates are present in the diet (FAO/WHO, 2002), (Monsen et al., 1978). Legumes and grains are the main sources of phytates, including soy beans (Schlemmer et al., 2009). Since soy represents a large part of the meat replacers, the absorbabilities of iron and zinc could be hindered. A sensitivity analysis (Appendix 6.E) shows that the optimal solutions are not sensitive for changes in the value of the required contents of iron and zinc in the chicken replacers. However, for the beef replacers the optimal solutions are very sensitive for changes in the required contents of zinc and iron. It should be investigated how components like phytates affect the required content of non-heme iron and zinc in the replacement products and how this can be integrated it in the model. Another suggestion is to examine the replacers within a broader dietary setting to better understand the effect of nutrient contributions outside the scope of the reference nutrients.

This study relies on a limited number of LCA studies for the environmental data of the ingredients. The consulted studies, however, have the same framework, being the applied (geographical) boundaries, functional unit and allocation method. Since the framework of existing LCA studies often deviate, including data from additional studies would entail more uncertainty. There is thus a need for harmonization of LCA methods, since it facilitates the comparison of future studies (Margni and Curran, 2012). 
The model included a limited number of ingredients, which were selected based on their nutritional value and based on recipes of a meat replacer producer (Averink, 2015). Certain forms of the ingredients were selected, depending on their general application in industry and the availability and quality of their data. For some ingredients, however, assumptions concerning their environmental impact were made. Since environmental impact data of iron, zinc and vitamin B12 fortifications were not available, we made an estimation of these data. A sensitivity analysis (Appendix 6.E) however shows that the model results are hardly sensitive to changes in the environmental impact values of the fortifications. This study could be expanded by including more ingredients, provided that the framework of the consulted LCA studies correspond.

\subsection{Conclusion}

This study proposes a linear programming model in order to compose nutrient equivalent substitutes for meat that can be used as direct replacements and lower the environmental impact of our diet. The results suggest, that a shift from meat to plant-based products can lead to significant reductions in terms of the environmental impact, without losing the nutritional benefits of meat. The vegan replacers have the biggest potential to realize environmental impact reductions with regards to climate change, land use and fossil fuel depletion. With respect to water use, the insect-based replacers show the most promising reduction potential. The level of reduction further depends on the kind of meat that is replaced, with beef having the largest environmental impact among meat types. Beef provides high levels of important nutrients that cannot easily be achieved by other products. It is therefore not possible to compose a nutritionally equivalent product to beef without the use of fortifications. For future research, more integrated and comprehensive analyses of the role of replacers within the diet and the supply systems of ingredients are required. In this context, more standardized data and better knowledge with regards to the environmental impact of different products and geographical regions are needed. 


\section{A Appendix - Required Contents of Nutrients}

The required nutrient contents for the meat replacers are based on the nutritional value of the references chicken $(k=1)$ and beef $(k=2)$. The references' contents of protein, iron, vitamin B12 and zinc are used as lower bounds $\left(r n_{j k}\right)$ for the meat replacer.

The required contents of essential amino acids are based on the human requirements for these amino acids. They are calculated by multiplying the human requirement of the amino acid (mg/g protein) with the protein content of the references chicken and beef.

Iron can be found in two forms: heme iron and non-heme iron, of which the latter is less absorbable by the human body (Bender, 1992). Meat contains both forms, but in nonmeat products only non-heme iron is present (Monsen et al., 1978). The required amount of iron $\left(r c_{\text {iron, } k}\right)$ is expressed in terms of non-heme iron. It is calculated by summing the amount of non-heme iron with the amount of heme iron in the reference $k$, multiplied with a conversion factor to take the difference in absorbability into account:

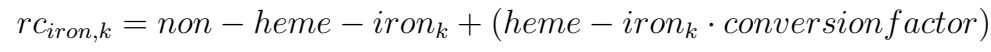

The conversion factor is calculated by dividing the absorbability of heme iron (23\% (Monsen et al., 1978)) with the absorbability of non-heme iron (8\% (Monsen et al., 1978)).

Table 6.6 shows the considered nutrients, their corresponding index $j$ and their required amount in the meat replacers, both when chicken and beef are used as reference.

Table 6.6: Considered nutrients $(j)$, their units and their required content in the meat replacer, both when chicken and beef are used as references.

\begin{tabular}{lllll}
\hline $\mathrm{j}$ & Name & Unit & $\begin{array}{l}\text { Reference = chicken } \\
\left(r n_{j, 1}\right)\end{array}$ & $\begin{array}{l}\text { Reference = beef } \\
\left(r n_{j, 2}\right)\end{array}$ \\
\hline 1 & Energy & $\mathrm{kJ} / 100 \mathrm{~g}$ & 667 & 679 \\
2 & Protein & $\mathrm{g} / 100 \mathrm{~g}$ & 30.9 & 29.7 \\
3 & Isoleucine & $\mathrm{g} / 100 \mathrm{~g}$ & 0.93 & 0.89 \\
4 & Leucine & $\mathrm{g} / 100 \mathrm{~g}$ & 1.82 & 1.75 \\
5 & Lysine & $\mathrm{g} / 100 \mathrm{~g}$ & 1.39 & 1.34 \\
6 & Methionine + cysteine & $\mathrm{g} / 100 \mathrm{~g}$ & 0.68 & 0.65 \\
7 & Phenylalanine + tyrosine & $\mathrm{g} / 100 \mathrm{~g}$ & 1.17 & 1.13 \\
8 & Threonine & $\mathrm{g} / 100 \mathrm{~g}$ & 0.71 & 0.68 \\
9 & Tryptophan & $\mathrm{g} / 100 \mathrm{~g}$ & 0.19 & 0.18 \\
10 & Valine & $\mathrm{g} / 100 \mathrm{~g}$ & 1.21 & 1.16 \\
11 & Iron (non-heme) & $\mathrm{mg} / 100 \mathrm{~g}$ & 2.03 & 6.46 \\
12 & Vitamin B12 & $\mu \mathrm{g} / 100 \mathrm{~g}$ & 0.29 & 2.11 \\
13 & Zinc & $\mathrm{mg} / 100 \mathrm{~g}$ & 0.74 & 6.45 \\
\hline
\end{tabular}




\section{B Appendix - Life Cycle Assessment and Environ- mental Impact Indicators}

Life Cycle Assessment (LCA) is a method to determine the environmental impact of a product's entire life cycle, from agricultural production ('cradle') to the consumption and the recycling of the product ('grave'). An LCA consists of four steps: goal \& scope definition, inventory analysis, impact assessment and interpretation (Mogensen et al., 2009). The goal and scope definition aims to define the studied supply chain, the functional unit, the system boundaries, the impact indicators and applied methods (Sauer, 2012). The life cycle inventory contains all the data related to the used resources and emissions to the environment that occur during the life cycle of a product. The data are quantified in relation to the defined functional unit (Finnveden et al., 2009), for example one kilogram of end product (Sauer, 2012). The impact assessment aims to evaluate the environmental impact of the resources and emissions that are reported in the inventory analysis (Margni and Curran, 2012). The environmental impact is expressed in terms of environmental impact indicators, for which values are quantified. In the interpretation step of the LCA, the results of the inventory analysis and the impact assessment are evaluated to take decisions related to the goal of the LCA (Margni and Curran, 2012).

LCA studies of food products often deviate in terms of selected impact indicators, functional unit, system boundaries and applied methods. This can cause different results among LCA studies that investigate the same product or process (Margni and Curran, 2012). When comparing different LCA studies, it should be made sure that the functional unit, system boundaries and allocation method correspond (de Vries and de Boer, 2010). For example, when the system boundary of one study includes all the life cycle stages from agricultural production to consumption (from cradle to grave) and another study only includes the agricultural phase (from cradle to farm gate), their results cannot be compared.

Moreover, there is no standardized method for selecting LCA impact indicators for a certain product (Cerutti et al., 2011). Therefore, we analyzed existing LCA studies of food products and their impact indicators, in which we characterized the quality and applicability of the indicators. Table 6.7 shows the analyzed studies, the applied impact indicators and their characteristics. 
Table 6.7: Reviewed studies and their applied impact indicators

\begin{tabular}{|c|c|c|c|c|c|c|c|c|c|c|c|c|c|c|c|c|c|c|c|c|}
\hline & 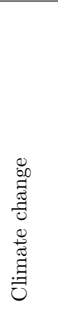 & 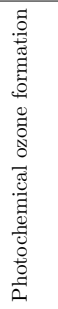 & 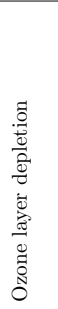 & 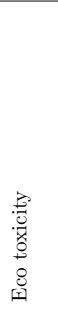 & 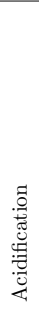 & 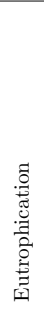 & 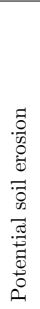 & 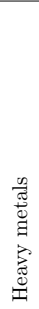 & 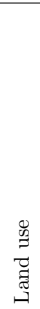 & 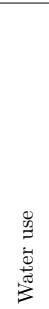 & 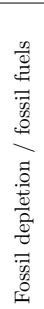 & 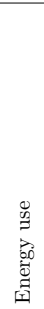 & 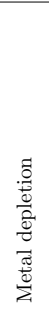 & 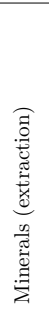 & 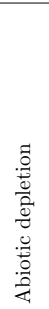 & 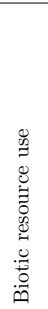 & 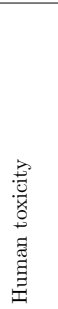 & 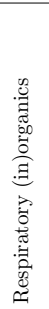 & 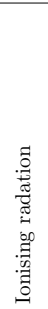 & 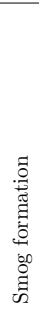 \\
\hline $\begin{array}{l}\text { Smetana et al. } \\
(2015)\end{array}$ & $\mathrm{x}$ & $\mathrm{x}$ & $\mathrm{x}$ & $\mathrm{x}$ & $\mathrm{x}$ & $\mathrm{x}$ & & & $\mathrm{x}$ & & $\mathrm{x}$ & & $\mathrm{x}$ & & & & $\mathrm{x}$ & & $\mathrm{x}$ & \\
\hline $\begin{array}{l}\text { Hallström et al. } \\
(2015)\end{array}$ & $\mathrm{x}$ & & & & & & & & $\mathrm{x}$ & & & & & & & & & & & \\
\hline Meier et al. (2015) & $\mathrm{x}$ & & $\mathrm{x}$ & & $\mathrm{x}$ & $\mathrm{x}$ & & $\mathrm{x}$ & $\mathrm{x}$ & $\mathrm{x}$ & $\mathrm{x}$ & $\mathrm{x}$ & & & & & & & & \\
\hline Avadí et al. (2014) & $\mathrm{x}$ & & & & $\mathrm{x}$ & $\mathrm{x}$ & & & $\mathrm{x}$ & $\mathrm{x}$ & $\mathrm{x}$ & $\mathrm{x}$ & $\mathrm{x}$ & & & $\mathrm{x}$ & $\mathrm{x}$ & & & \\
\hline Foster et al. (2014) & $\mathrm{x}$ & $\mathrm{x}$ & & & $\mathrm{x}$ & $\mathrm{x}$ & & & $\mathrm{x}$ & $\mathrm{x}$ & & & & & & & & & & \\
\hline $\begin{array}{l}\text { van Dooren et al. } \\
\text { (2014) }\end{array}$ & $\mathrm{x}$ & & & & & & & & $\mathrm{x}$ & & & & & & & & & & & \\
\hline $\begin{array}{l}\text { Van Kernebeek } \\
\text { et al. }(2014)\end{array}$ & $\mathrm{x}$ & & & & & & & & $\mathrm{x}$ & & & & & & & & & & & \\
\hline $\begin{array}{l}\text { Castellini et al. } \\
(2012)\end{array}$ & $\mathrm{x}$ & & & & $\mathrm{x}$ & $\mathrm{x}$ & & & & & $\mathrm{x}$ & & & & & & & & & \\
\hline Ingwersen (2012) & $\mathrm{x}$ & $\mathrm{x}$ & & $\mathrm{x}$ & $\mathrm{x}$ & $\mathrm{x}$ & $\mathrm{x}$ & & & $\mathrm{x}$ & & $\mathrm{x}$ & & & & & $\mathrm{x}$ & & & $\mathrm{x}$ \\
\hline $\begin{array}{l}\text { Nijdam et al. } \\
(2012)\end{array}$ & $\mathrm{x}$ & & & & & & & & $\mathrm{x}$ & & & & & & & & & & & \\
\hline $\begin{array}{l}\text { Pardo and Zufía } \\
(2012)\end{array}$ & $\mathrm{x}$ & $\mathrm{x}$ & & & $\mathrm{x}$ & $\mathrm{x}$ & & & & & & & & & & & & & & \\
\hline $\begin{array}{l}\text { de Vries and } \\
\text { de Boer (2010) }\end{array}$ & $\mathrm{x}$ & & & & $\mathrm{x}$ & $\mathrm{x}$ & & & $\mathrm{x}$ & & & & & & & & & & & \\
\hline Davis et al. (2010) & $\mathrm{x}$ & & $\mathrm{x}$ & & $\mathrm{x}$ & $\mathrm{x}$ & & & $\mathrm{x}$ & & & $\mathrm{x}$ & & & & & & & & \\
\hline Blonk et al. (2008) & $\mathrm{x}$ & & & & & & & & $\mathrm{x}$ & & $\mathrm{x}$ & & & & & & & & & \\
\hline $\begin{array}{l}\text { Weidema and } \\
\text { Eder }(2008)\end{array}$ & $\mathrm{x}$ & $\mathrm{x}$ & $\mathrm{x}$ & $\mathrm{x}$ & $\mathrm{x}$ & $\mathrm{x}$ & & & $\mathrm{x}$ & $\mathrm{x}$ & & $\mathrm{x}$ & & $\mathrm{x}$ & & & $\mathrm{x}$ & $\mathrm{x}$ & & \\
\hline Sim et al. (2007) & $\mathrm{x}$ & $\mathrm{x}$ & $\mathrm{x}$ & $\mathrm{x}$ & $\mathrm{x}$ & $\mathrm{x}$ & & & & & & & & & $\mathrm{x}$ & & $\mathrm{x}$ & & & \\
\hline $\begin{array}{l}\text { Baroni et al. } \\
(2007)\end{array}$ & $\mathrm{x}$ & & $\mathrm{x}$ & $\mathrm{x}$ & $\mathrm{x}$ & $\mathrm{x}$ & & & $\mathrm{x}$ & & $\mathrm{x}$ & & & $\mathrm{x}$ & & & $\mathrm{x}$ & $\mathrm{x}$ & & \\
\hline $\begin{array}{l}\text { Tukker and } \\
\text { Jansen (2006) }\end{array}$ & $\mathrm{x}$ & $\mathrm{x}$ & & & $\mathrm{x}$ & $\mathrm{x}$ & & & & & & & & & & & & & & \\
\hline $\begin{array}{l}\text { Zhu and van } \\
\text { Ierland (2004) }\end{array}$ & $\mathrm{x}$ & & & $\mathrm{x}$ & $\mathrm{x}$ & $\mathrm{x}$ & & & $\mathrm{x}$ & $\mathrm{x}$ & & & & & & & $\mathrm{x}$ & & & \\
\hline Usage frequency & 19 & 7 & 6 & 6 & 14 & 14 & 1 & 1 & 14 & 6 & 6 & 5 & 2 & 2 & 1 & 1 & 7 & 2 & 1 & 1 \\
\hline $\begin{array}{l}\text { Affecting AoP } \\
(\mathbf{H}, \mathbf{E}, \mathbf{R})^{*}\end{array}$ & $\mathrm{H}, \mathrm{E}$ & $\mathrm{H}, \mathrm{E}$ & $\mathrm{H}, \mathrm{E}$ & $\mathrm{H}, \mathrm{E}$ & $\mathrm{E}$ & $\mathrm{E}$ & $\mathrm{E}$ & $\mathrm{E}$ & $\mathrm{R}$ & $\mathrm{R}$ & $\mathrm{R}$ & $\mathrm{R}$ & $\mathrm{R}$ & $\mathrm{R}$ & $\mathrm{R}$ & $\mathrm{R}$ & $\mathrm{H}$ & $\mathrm{H}$ & $\mathrm{H}$ & $\mathrm{H}$ \\
\hline $\begin{array}{l}\text { Quality } \\
\text { Category } \\
\text { Hauschild }\end{array}$ & 1 & - & 1 & $2-3$ & 2 & 2 & - & - & 3 & 2 & 2 & - & - & - & 2 & 2 & $2-3$ & $1-2$ & 2 & - \\
\hline $\begin{array}{l}\text { Level of impact } \\
\text { (Global } \\
\text { (G)/Local (L)) }\end{array}$ & G & - & G & - & $\mathrm{L}$ & $\mathrm{L}$ & $\mathrm{L}$ & $\mathrm{L}$ & - & - & - & - & - & - & - & - & - & - & - & - \\
\hline $\begin{array}{l}\text { Related with } \\
\text { food production }\end{array}$ & $\mathrm{x}$ & $\mathrm{x}$ & & & $\mathrm{x}$ & $\mathrm{x}$ & & & $\mathrm{x}$ & $\mathrm{x}$ & $\mathrm{x}$ & $\mathrm{x}$ & & & & & & & & \\
\hline
\end{tabular}

Frequently applied indicators are useful since their results can be compared with results of previously executed studies. Similarly, they are reproducible for future studies. As can 
be seen in Table 6.7, climate change is used in all the investigated LCA studies. After that, land use, acidification and eutrophication were the most frequently used indicators. Ozone layer depletion, photochemical ozone formation, eco toxicity, water use and energy use where less frequently used, but also appear in a considerable number of studies. Each impact indicator affects one or more Areas of Protection (AoPs), namely natural resources, natural environment and human health (Margni and Curran, 2012). Table 6.7 indicates for each impact indicator which $\mathrm{AoP}(\mathrm{s})$ they affect. Multiple impact assessment methods exist and they often deviate from each other. Hauschild et al. (2013) investigate for a number of impact indicators which assessment method is most suitable and if the quality of this method is sufficient to be recommended. If the methods are recommended, they are classified in three categories, based on their quality: (1) recommended and satisfactory, (2) recommended but in need of some improvements and (3) recommended but to be applied with caution. Impact indicators for which a model of category 1 exists are climate change and ozone layer depletion. Impact indicators for which only models of category 2 exist are photochemical ozone formation, acidification, eutrophication and resource depletion (water and mineral resources) (Hauschild et al., 2013). The most frequently used impact indicators correspond with the ones that can be assessed with a method of category 1 or 2, except for land use. Land use is classified in the third category, so the existing method should be applied with caution. Some impact indicators influence the global environment and some indicators influence only the local environment, which makes their impact highly dependent on local conditions. Impact indicators that influence the global environment are climate change and ozone layer depletion (Finnveden et al., 2009). The fact that they influence the global environment, means that they can be easily compared and applied to other products and other countries. According to Davis et al. (2010), some impact indicators are particularly related to food production, including climate change, photochemical ozone formation, acidification, eutrophication, energy use and fossil fuel depletion (Davis et al., 2010). Water use is also related to food since agriculture alone already contributes for $70-85 \%$ to the global water footprint (Smetana et al., 2015). Additionally, food processing operations require a lot of energy and water (Mishra et al., 2012). Land use is an important impact category when it comes to feed and food (Smetana et al., 2015) and as can be seen from Table 6.7, it is used in a large number of LCA studies of food products. 


\section{C Appendix - Nutritional and Environmental Data}

Table 6.8 provides the environmental data of the ingredients and Table 6.10 provides the environmental data of the references. Table 6.9 provides the nutritional data of the model ingredients.

Table 6.8: Environmental data of model ingredients.

\begin{tabular}{|c|c|c|c|c|c|}
\hline & $\begin{array}{l}\text { Environmental impact (per kg) } \\
\text { Unit }\end{array}$ & $\begin{array}{l}\text { Climate change } \\
\mathrm{kg} \mathrm{CO}_{2} \text { eq }\end{array}$ & $\begin{array}{l}\text { Land use } \\
\mathrm{m}^{2} / \text { year }\end{array}$ & $\begin{array}{l}\text { Water use } \\
\mathrm{m}^{3}\end{array}$ & $\begin{array}{l}\text { Fossil fuel depletion } \\
\text { MJ }\end{array}$ \\
\hline $\mathrm{X} 1$ & Chickpea flour & 0.99 & 8.42 & $0.266^{a}$ & 13.82 \\
\hline $\mathrm{X} 2$ & Chickpea protein concentrate & 1.45 & 9.64 & 0.266 & 29.89 \\
\hline $\mathrm{X} 3$ & Chickpea protein isolate & 3.13 & 18.42 & 0.266 & 51.16 \\
\hline $\mathrm{X} 4$ & Kidney bean flour & 1.47 & 3.69 & $0.148^{a}$ & 14.62 \\
\hline $\mathrm{X} 5$ & Kidney bean protein concentrate & 2.16 & 4.22 & 0.148 & 31.61 \\
\hline $\mathrm{X} 6$ & Kidney bean protein isolate & 4.65 & 8.07 & 0.148 & 54.11 \\
\hline $\mathrm{X} 7$ & Lentil flour & 1.00 & 7.71 & $0.580^{a}$ & 12.93 \\
\hline $\mathrm{X} 8$ & Lentil protein concentrate & 1.47 & 8.83 & 0.580 & 27.96 \\
\hline X9 & Lentil protein isolate & 3.16 & 16.87 & 0.580 & 47.86 \\
\hline $\mathrm{X} 10$ & Lupine flour & 1.06 & 7.77 & $0.173^{a}$ & 10.48 \\
\hline $\mathrm{X} 11$ & Lupine protein concentrate & 1.24 & 9.16 & 0.173 & 12.34 \\
\hline $\mathrm{X} 12$ & Lupine protein isolate & 3.28 & 15.66 & 0.025 & 38.47 \\
\hline $\mathrm{X} 13$ & Pea flour & 0.62 & 2.79 & $0.173^{a}$ & 6.24 \\
\hline $\mathrm{X} 14$ & Pea protein concentrate & 1.12 & 5.03 & 0.173 & 11.25 \\
\hline $\mathrm{X} 15$ & Pea protein isolate & 3.16 & 8.58 & 0.173 & 39.72 \\
\hline $\mathrm{X} 16$ & Soy flour & 0.82 & 4.52 & $0.083^{a}$ & 8.69 \\
\hline $\mathrm{X} 17$ & Soy protein concentrate & 1.21 & 5.17 & 0.083 & 18.79 \\
\hline $\mathrm{X} 18$ & Soy protein isolate & 2.60 & 9.89 & 0.083 & 32.16 \\
\hline $\mathrm{X} 19$ & Wheat flour & 0.63 & 1.69 & $0.347^{a}$ & 5.12 \\
\hline $\mathrm{X} 20$ & Wheat protein concentrate & 0.92 & 1.929 & 0.347 & 11.06 \\
\hline $\mathrm{X} 21$ & Wheat protein isolate (gluten) & 2.93 & 4.64 & 0.785 & 35.28 \\
\hline $\mathrm{X} 22$ & Eggs & $4.25^{c}$ & $5.03^{c}$ & $0.244^{b}$ & 13.50 \\
\hline $\mathrm{X} 23$ & Cheese & $7.80^{d}$ & $6.61^{d}$ & $0.439^{b}$ & $38.21^{d}$ \\
\hline $\mathrm{X} 24$ & Meal worms & $2.65^{e}$ & $3.56^{e}$ & $0.003^{b}$ & $33.68^{e}$ \\
\hline $\mathrm{X} 25$ & Super worms & $2.65^{e}$ & $3.56^{e}$ & $0.003^{b}$ & $33.68^{e}$ \\
\hline $\mathrm{X} 26$ & Water & 0.00 & 0.00 & 0.000 & 0.00 \\
\hline $\mathrm{X} 27$ & Iron fortification & 0.30 & 2.00 & 0.010 & 5.00 \\
\hline $\mathrm{X} 28$ & B12 fortification & 0.30 & 2.00 & 0.010 & 5.00 \\
\hline $\mathrm{X} 29$ & Zinc fortification & 0.30 & 2.00 & 0.010 & 5.00 \\
\hline
\end{tabular}

Source: (Broekema and Smale, 2011), except when indicated with a letter. a: (Mekonnen and Hoekstra, 2011),

b: (Mekonnen and Hoekstra, 2013), c: (de Vries and de Boer, 2010), d: (Van Middelaar et al., 2011),

e: (Oonincx and De Boer, 2012) 
Table 6.9: Nutritional value of the model ingredients

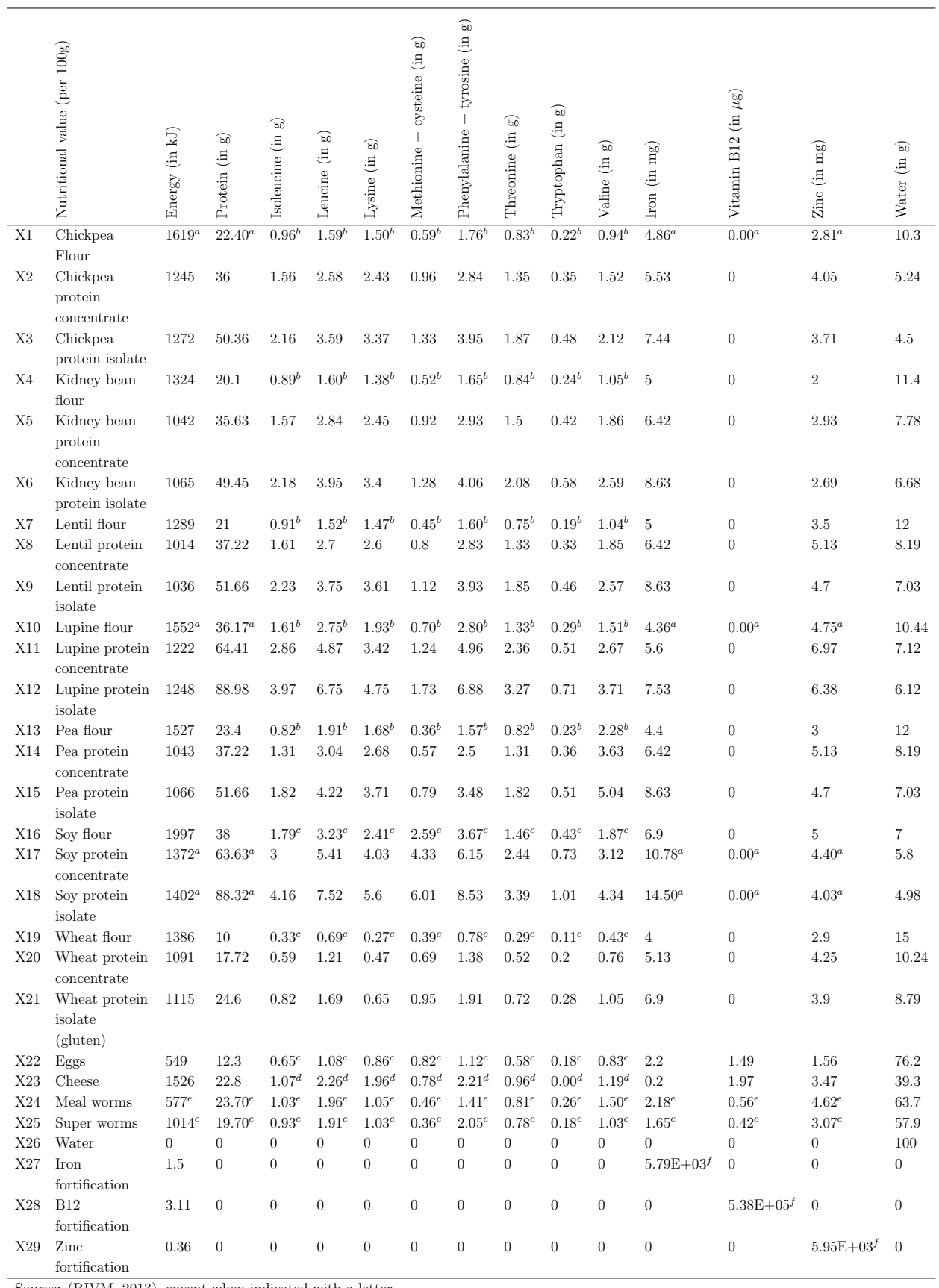

Source: (RIVM, 2013), except when indicated with a letter.

a:(USDA, 2016), b: (Broekema and Smale, 2011), c: (Friedman, 1996), d:(Davis et al., 1994), e: (FAO/INFOODS, 2013), f: (Flindall, 2016) 
Table 6.10: Environmental data of chicken and beef

\begin{tabular}{lccc}
\hline & Unit & Chicken & Beef \\
\hline Climate change & $\mathrm{kg} \mathrm{CO}_{2} \mathrm{eq} / \mathrm{kg}$ & 2.51 & 7.53 \\
Land use & $\mathrm{m}^{2} /$ year$/ \mathrm{kg}$ & 4.79 & 5.96 \\
Water use & $\mathrm{m}^{3} / \mathrm{kg}$ & 0.049 & 0.064 \\
Fossil depletion & $\mathrm{MJ} / \mathrm{kg}$ & 17.81 & 20.39 \\
\hline
\end{tabular}

Source: (Blonk et al., 2007) 


\section{D Appendix - Payoff Matrices}

Table 6.11 shows the payoff matrices for the four environmental impact indicators. The matrices for the vegetarian and the vegan chicken replacer are identical, because the optimal solutions for both chicken replacers are the same. The highest values (underlined) of $Z_{c c}, Z_{l u}$ and $Z_{f d}$ occur when water use $\left(Z_{w u}\right)$ is minimised, except for the fortificationfree chicken replacer, where the highest value of $Z_{l u}$ occurs when fossil fuel depletion $\left(Z_{f d}\right)$ is minimised. The minimisation of water use therefore has the largest tradeoffs. Also for the beef replacers the water use has the largest tradeoffs.

Table 6.11: Payoff matrices for the feasible meat replacers

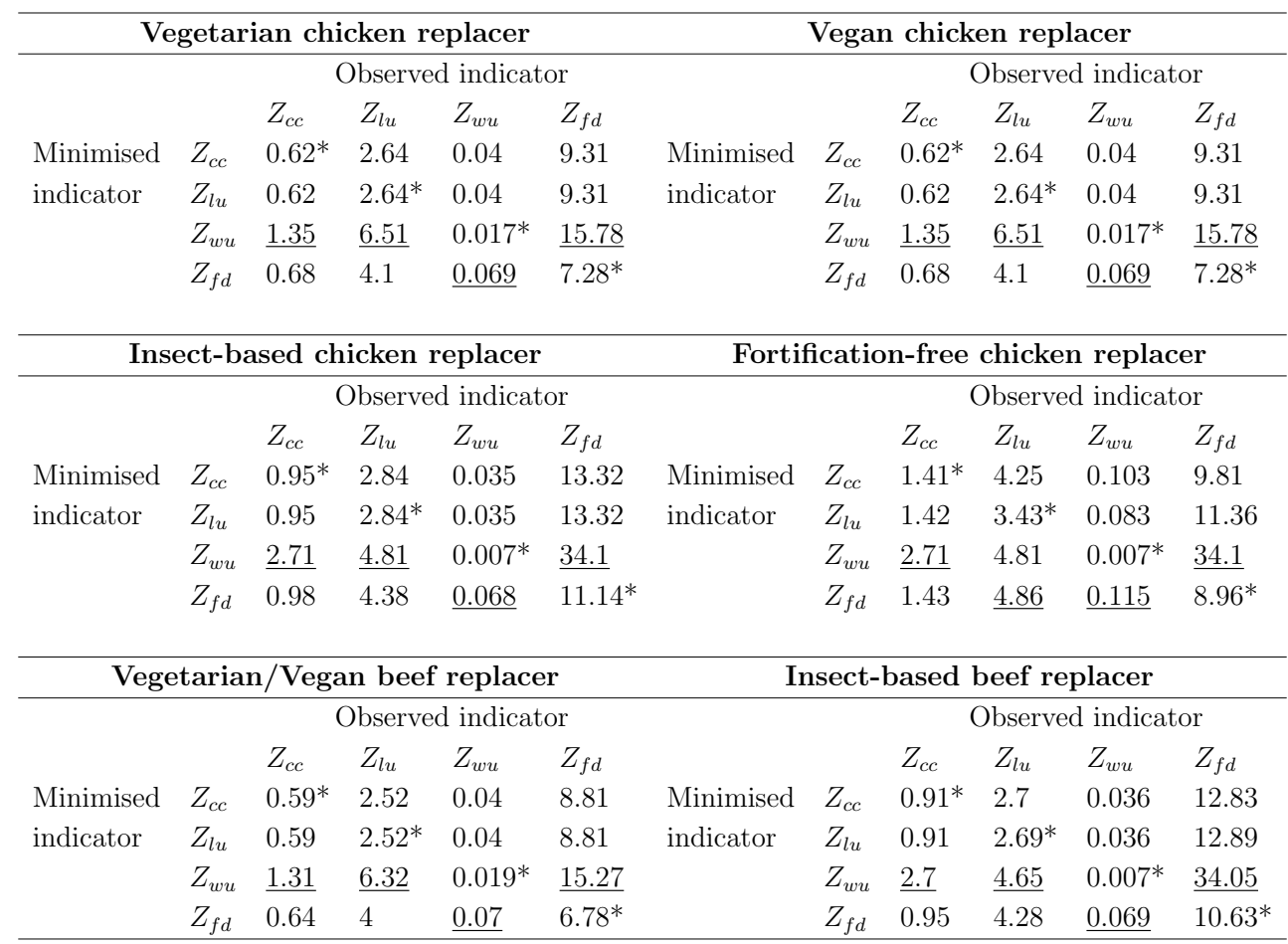




\section{E Appendix - Sensitivity Analysis}

\section{Cost coefficient ranges of fortifications}

This sensitivity analysis investigates the effect of changing the environmental impact values of the fortifications on the model results. The cost coefficient ranges of the environmental impact values are determined, which represent the ranges of values for which the solution stays optimal (Hendriks, 2013). The upper bound of the values thus represent the maximum value for which the solution stays optimal. Table 6.12 shows the cost coefficient ranges of the environmental impact values of vitamin B12 for the vegetarian/vegan chicken replacer.

Table 6.12: Cost coefficient ranges of environmental impact values of vitamin B12 fortification for the vegetarian/vegan chicken replacer

\begin{tabular}{|c|c|c|c|}
\hline & \multicolumn{3}{|c|}{ Cost coefficient ranges vitamin $\mathrm{B} 12$} \\
\hline & Current value & Lower bound & Upper bound \\
\hline $\mathrm{CC} \mathrm{(28)}\left(\mathrm{kg} \mathrm{CO}_{2} \mathrm{eq}\right)$ & 8 & 0 & $1.38 \mathrm{E}+06$ \\
\hline LU (28) $\left(\mathrm{m}^{2} /\right.$ year $)$ & 19 & 0 & $7.14 \mathrm{E}+05$ \\
\hline WU $(28)\left(\mathrm{m}^{3}\right)$ & 0.8 & 0 & $7.93 \mathrm{E}+04$ \\
\hline FD (28) (MJ) & 52 & 0 & $3.11 \mathrm{E}+06$ \\
\hline
\end{tabular}

Tables 6.13 to 6.15 show the cost coefficient ranges of the environmental impact values of iron, vitamin B12 and zinc for the vegetarian/vegan beef replacer. It can be seen from the tables, that the upper bounds of the environmental values are very high compared with the current values. The composition of the meat replacers are therefore not sensitive for changes in cost coefficients and are thus quite robust.

Table 6.13: Cost coefficient ranges of environmental impact values of iron fortification for the vegetarian/vegan beef replacer

\begin{tabular}{|c|c|c|c|}
\hline & \multicolumn{3}{|c|}{ Cost coefficient ranges iron } \\
\hline & Current value & Lower bound & Upper bound \\
\hline $\mathrm{CC} \mathrm{(27)}\left(\mathrm{kg} \mathrm{CO}_{2}\right.$ eq $)$ & 8 & 0 & $2.15 \mathrm{E}+03$ \\
\hline LU (27) $\left(\mathrm{m}^{2} /\right.$ year $)$ & 19 & 0 & $7.26 \mathrm{E}+03$ \\
\hline WU $(27)\left(\mathrm{m}^{3}\right)$ & 0.8 & 0 & $3.92 \mathrm{E}+01$ \\
\hline FD (27) (MJ) & 52 & 0 & $6.98 \mathrm{E}+03$ \\
\hline
\end{tabular}


Table 6.14: Cost coefficient ranges of environmental impact values of vitamin B12 fortification for the vegetarian/vegan beef replacer

\begin{tabular}{|c|c|c|c|}
\hline & \multicolumn{3}{|c|}{ Cost coefficient ranges vitamin B12 } \\
\hline & Current value & Lower bound & Upper bound \\
\hline $\mathrm{CC} \mathrm{(28)}\left(\mathrm{kg} \mathrm{CO} \mathrm{CO}_{2}\right.$ eq $)$ & 8 & 0 & $1.38 \mathrm{E}+06$ \\
\hline LU (28) $\left(\mathrm{m}^{2} /\right.$ year $)$ & 19 & 0 & $7.21 \mathrm{E}+05$ \\
\hline WU $(28)\left(\mathrm{m}^{3}\right)$ & 0.8 & 0 & $7.93 \mathrm{E}+04$ \\
\hline FD (28) (MJ) & 52 & 0 & $3.11 \mathrm{E}+06$ \\
\hline
\end{tabular}

Table 6.15: Cost coefficient ranges of environmental impact values of zinc fortification for the vegetarian/vegan beef replacer

\begin{tabular}{lccc}
\hline & \multicolumn{3}{c}{ Cost coefficient ranges zinc } \\
& Current value & Lower bound & Upper bound \\
\hline CC (29) (kg CO $\left.\mathbf{~}_{\mathbf{2}} \mathbf{~ e q}\right)$ & 8 & 0 & $3.73 \mathrm{E}+02$ \\
LU (29) $\left(\mathbf{m}^{2} /\right.$ year $)$ & 19 & 0 & $3.05 \mathrm{E}+03$ \\
WU (29) $\left(\mathbf{m}^{3}\right)$ & 0.8 & 0 & $1.30 \mathrm{E}+02$ \\
FD (29) $(\mathbf{M J})$ & 52 & 0 & $2.85 \mathrm{E}+04$ \\
\hline
\end{tabular}

\section{Sensitivity lower bounds of iron and zinc}

Since the absorbability of non-heme iron and zinc depend on the presence of certain components in the diet the required content of iron and zinc in the meat replacer are uncertain. The required contents represent the lower bounds of the constraints for iron and zinc. It is investigated how changes in these lower bounds affect the optimal solutions. To do this, the slacks and shadow prices are calculated. The slacks represent how much the lower bounds can be increased without affecting the optimal solution. The shadow prices represent the increase in the objective value when the lower bound is increased with one unit. Table 6.16 shows the current value, the slacks and the shadow prices of the lower bounds for the vegetarian/vegan chicken replacer. The shadow price is zero for both lower bounds and for all minimisations. This means that the zinc and iron constraints are both not binding (Hendriks, 2013). The slacks for both lower bounds are relatively high compared to the current values, so the optimal solutions are not very sensitive for changes in the value of the required content of iron and zinc. Table 6.17 shows the current value, the slacks and the shadow prices of the lower bounds for the vegetarian/vegan beef replacer. The slacks are zero for both lower bounds and for all minimisations. This means that the zinc and iron constraints are binding. The shadow prices are quite high compared 
to the current values, which means that the results are sensitive for changes in required contents of iron, zinc and vitamin B12.

Table 6.16: Slacks and shadow prices of the lower bounds of zinc and iron for the vegetarian/vegan chicken replacer

\begin{tabular}{lllllll}
\hline & & \multicolumn{4}{c}{ Minimised indicator } \\
\hline \multirow{2}{*}{ Required content iron $(\mathbf{m g} / \mathbf{1 0 0} \mathbf{g})$} & CC & LU & WU & FD \\
& & Current value & 2.03 & 2.03 & 2.03 & 2.03 \\
& Slack & 3.2 & 3.2 & 1.53 & 1.51 \\
& Shadow price & 0 & 0 & 0 & 0 \\
Required content zinc $(\mathbf{m g} / \mathbf{1 0 0} \mathbf{g})$ & Current value & 0.74 & 0.74 & 0.74 & 0.74 \\
& Slack & 1.36 & 1.36 & 2.2 & 2.22 \\
& Shadow price & 0 & 0 & 0 & 0 \\
\hline
\end{tabular}

Table 6.17: Slacks and shadow prices of the lower bounds of zinc and iron for the vegetarian/vegan beef replacer

\begin{tabular}{lllllll}
\hline & & \multicolumn{5}{c}{ Minimised Indicator } \\
& & CC & LU & WU & FD \\
\hline Required content iron $(\mathbf{m g} / \mathbf{1 0 0} \mathbf{g})$ & Current value & 6.46 & 6.46 & 6.46 & 6.46 \\
& Slack & 0 & 0 & 0 & 0 \\
& Shadow price & 0.22 & 0.62 & 0.02 & 2 \\
Required content zinc $(\mathbf{m g} / \mathbf{1 0 0} \mathbf{g})$ & Current value & 6.45 & 6.45 & 6.45 & 6.45 \\
& Slack & 0 & 0 & 0 & 0 \\
& Shadow price & 0.22 & 0.6 & 0.01 & 1.07 \\
\hline
\end{tabular}




\section{F Appendix - Compositions of the Meat Replac- ers}

Table 6.18 provides the composition of the chicken replacers and Table 6.19 provides the composition of the beef replacers.

Table 6.18: Compositions of the chicken replacers. Fortifications in $\mathrm{mg} / \mathrm{kg}$; all other ingredients in $\mathrm{g} / 100 \mathrm{~g}$

\begin{tabular}{llllllll}
\hline & & \multicolumn{2}{c}{ Vegetarian/Vegan } & \multicolumn{2}{c}{ Insect-based } & \multicolumn{2}{c}{ Fortification-free } \\
& & minsum & minmax & minsum & minmax & minsum & minmax \\
\hline X12 & Lupine protein isolate & - & 8.7 & - & 5.0 & - & 7.4 \\
X16 & Soy flour & 1.7 & 5.0 & - & - & - & 0.5 \\
X17 & Soy protein concentrate & 42.2 & 33.3 & 38.9 & 29.5 & 37.7 & 25.9 \\
X18 & Soy protein isolate & 3.8 & - & 2.9 & - & 5.1 & - \\
X22 & Eggs & - & - & - & - & 19.5 & 9.1 \\
X24 & Meal worms & - & - & 14.2 & 30.6 & - & 27.5 \\
X25 & Super worms & - & - & 1.1 & 2.4 & - & - \\
X26 & Water & 52.2 & 52.9 & 43.0 & 32.6 & 37.7 & 29.6 \\
X28 & B12 fortification & 0.54 & 0.54 & 0.38 & 0.20 & - & - \\
\hline
\end{tabular}

Table 6.19: Compositions of the beef replacers. Fortifications in $\mathrm{mg} / \mathrm{kg}$; all other ingredients in $\mathrm{g} / 100 \mathrm{~g}$.

\begin{tabular}{llllll}
\hline & & \multicolumn{2}{c}{ Vegetarian/Vegan } & \multicolumn{2}{c}{ Insect-based } \\
& & minsum & minmax & minsum & minmax \\
\hline X12 & Lupine protein isolate & - & 7.2 & - & 3.9 \\
X16 & Soy flour & 3.9 & 7.4 & - & - \\
X17 & Soy protein concentrate & 42.3 & 32.1 & 41.4 & 29.4 \\
X18 & Soy protein isolate & 1.5 & - & - & - \\
X24 & Meal worms & - & - & 9.7 & 25.0 \\
X25 & Super worms & - & - & 5.5 & 8.2 \\
X26 & Water & 52.2 & 53.1 & 43.4 & 33.4 \\
X27 & Iron fortification & 245 & 335 & 294 & 401 \\
X28 & B12 fortification & 3.92 & 3.92 & 3.78 & 3.60 \\
X29 & Zinc fortification & 728 & 706 & 674 & 588 \\
\hline
\end{tabular}




\section{G Appendix - Environmental Impact of Soy}

In this study, soy emerges as one of the main ingredients for the meat replacer due to its high protein quality and low environmental impact values. However, the topic of soy production and its environmental impact are subject to controversy in the scientific literature and have been increasingly addressed over recent years. One of the main concerns associated with the production of soy is its role as a main driver of deforestation and its relation to direct and indirect land use change in areas such as Brazil's Amazon Basin, resulting in a substantial loss of Amazonian rainforest (Costa et al., 2007; Arima et al., 2011). Brazil's Amazonian rainforest plays an important part in mitigating climate change by storing carbon, which is released as a result of deforestation (Fearnside, 2018). Research suggests, that the destruction of the forest is has a significant impact on the Amazonian ecosystem and thus on biodiversity and climate regulation (Coe et al., 2013). Brazil is one of the main exporters of soy, alongside Argentina and the US, and supplies a large amount of the demand of feed for livestock systems in Europe and Asia (Billen et al., 2014). The growing demand of soy for global livestock, food as well as domestic biodiesel production in Brazil puts further pressure and incentives to increase soy production within the Amazon region (Costa et al., 2007; Lima et al., 2011).

While there is a concern regarding the environmental impact of soy production within the Amazon Basin, studies also show that the majority of deforestation can be attributed to pasture expansion (Barona et al., 2010; Macedo et al., 2012). However, these studies also show indications for linkages between increased soy production in the Mato Grosso region (former pasture area) and the pasture expansion further north, making soy production an underlying factor of deforestation. Furthermore, it should be noted that often, even though the land was cleared for soy, soy production only starts at a later stage while other crops or pastures are used to prepare the land (Lima et al., 2011; Gasparri et al., 2013), making it more difficult to allocate deforestation directly to soy production. When comparing land occupation by soybean production with pasture land occupation, Costa et al. (2007) show differences in the climate impact of both cases, with lower precipitation rates for soy due to larger surface albedo and lower evapotranspiration. Similar results are presented in the study of Sampaio et al. (2007).

Research shows that the environmental impact, associated to land use change of soy, as well as beef production, largely depends on the region as well as the methodology used to quantify land use change (Persson et al., 2014). Furthermore, there are large differences between individual production and transport scenarios. Da Silva et al. (2010) present differences in the environmental impact of soybean production in the Central West and the South of Brazil. Their results show, that impact values are generally higher in the Central West due to deforestation and transportation. A study by Castanheira and Freire (2013) also found variations between different cultivation systems of soy, in particular related to 
different land use change scenarios. Evaluating 45 different scenarios, the research shows variations of almost 150 to 200 times the amount of greenhouse gas emissions, with the lowest values found for degraded grassland in Argentina and the highest values related to systems associated with tropical rainforest conversion. Gasparri et al. (2013), however, find similar links between deforestation and soybean production in Northern Argentina's Dry Chaco, establishing soy as one of the main drivers of deforestation in the region. Similarly to Brazil, the deforestation in Argentina raises concerns in terms of the destruction of natural habitats as well as carbon storage (Viglizzo et al., 2011).

While there is evidence of soy being one of the main drivers of deforestation in South America, it should be noted that most of the soy produced in countries such as Brazil or Argentina is used for protein rich feed production to supply a growing demand of meat (Billen et al., 2014; Viglizzo et al., 2011). About $75 \%$ of soy is used to feed livestock (Aiking, 2011). In fact, soybean, produced in Argentina, Brazil and the USA with over half of the crop being genetically modified, is one of the main sources for animal feed, due to its high quality protein composition (FAO, 2004). However, the conversion of plant-based protein to animal protein is inefficient, with the possible exception of grassfed livestock (Sabaté and Soret, 2014). Research suggests that the conversion from plant to animal protein results in losses of around $85 \%$ with only $15 \%$ of the protein reaching the human consumption stage (Aiking, 2011). It should, however, be recognised that livestock systems possess the advantage to salvage, for human indigestible, by-products (soy-bean hulls) that otherwise might turn to waste and turn it into high quality protein (Wu et al., 2014). In this context, Van Kernebeek et al. (2016) argues that land is used most efficiently if $12 \%$ of the human dietary protein intake comes from animal sources that convert such by-products. Furthermore, the study of Sasu-Boakye et al. (2014) show, that using locally sourced feed can have a positive effect on the environmental impact of livestock production in Europe in comparison to feed based on soy sourced from South America.

Based on these results, it is important to note that there are substantial variations in the environmental impact of soy and an increased production of soy alongside an increase in the consumption of meat is likely to pose a threat for the environment. However, so far, only a very small amount of soy protein is used for human consumption, with only about $2-3 \%$ of total soy production being used for human food (Day, 2013). A reduction in intensive livestock production could reduce the demand for soy as feed and thus free agricultural land currently devoted to the production of animal feed. This newly available land could then be used to supply plant-based protein for human consumption without requiring further land expansions and/or land use changes. However, for future scientific research there is a need to increasingly link the consumption and supply of food products while also looking into the different supply configurations within the system. 



\section{Chapter 7}

\section{Digesting the Pieces}

A general discussion and conclusion 


\subsection{Lasting Flavours - A Bite-Sized Conclusion}

The research in this thesis shows that Operations Research approaches can facilitate a rethinking of today's food system and help in finding solutions for a more sustainable future. By investigating different aspects of the system, the thesis addresses problems arising at the strategic, operational and individual decision level and provides tailored solutions for different agents in the system. As a result, the research covers a wide range of decision problems, varying in size and complexity. This large variety of problems reflects the multifaceted nature of challenges in the food system and demonstrates the ability of Operations Research to contribute to decision making on different levels in the system. Moreover, synergies can be identified between Operations Research and certain food related disciplines, such as human nutrition, when working towards practical solutions for a more sustainable future. Based on this, there is a lasting need and further potential for Operations Research approaches providing tools for decision support and analysis in the context of the food system.

Building on the insights gained in the previous chapters, the following sections provide a breakdown and discussion of the main findings as well as a synthesis of the scientific and practical contributions of this thesis in the form of integrated findings. The final section concludes this thesis by identifying possible directions for future research.

\subsection{Catabolism - Breaking down the Compounds}

Given the broad range of problems covered in this thesis, the findings and conclusions that can be derived from this research are manifold, linking to different aspects in the system. By decomposing the system into smaller components, this section highlights the main findings and discusses possible implications and limitations of this research. For ease of readability, the structure of this section follows the general framework of the thesis presented in Figure 1.1 of Chapter 1.

\subsubsection{Decisions - Supply Chain}

Supply chain decisions play a crucial role in the context of the food system and may relate to a large variety of production and distribution activities. Environmental and societal pressures demand a rethinking of these activities that still allows for economically viable solutions. Two decision levels have been considered in this context investigating possible alternatives at the strategic and the operational level of the system. 


\section{Network Perspective}

Looking at the system from a network perspective provides a number of strategic insights concerning the setup of the system. By modelling the system as an extensive web of global supply chain activities that features multiple echelons and interlinkages between individual supply chains, it is possible to get a more holistic understanding of product relationships, improvement opportunities and the allocation of pressures in the system (as shown in Chapter 2 and 3).

Relationships between products are a crucial factor when rethinking the system configuration as interlinkages between products and supply chains may hamper the ability to remove certain stages from the system without affecting other aspects. This applies in particular to resource intensive subsystems, such as livestock systems, comprising several intermediary products, processing steps and product side-streams.

Supply chain decisions in the food system are further constrained by available infrastructure and preexisting climatic and geographical conditions within a country. This holds in particular for the agricultural production phase where climate and soil compositions determine cultivation practices and necessary resource inputs in the form of, for example, irrigation or fertiliser usage in order to meet the specific product requirements.

Given the different environmental profiles of production activities and locations, the optimal setup of the system largely depends on the choice of objective with respect to different environmental impact indicators. Globally optimal solutions capable of reducing the overall impact of the system with respect to a certain impact indicator may however put a strain on the resources, production capacities and local environment of individual production locations, shifting the burden from one country to the next.

Considering the different stages in the network, the agricultural production phase can be identified as the main contributor to cost and environmental impacts in the system, followed by the processing phase, while transportation only has a minor contribution. This does however not indicate a larger improvement potential at the agricultural production phase, as certain impacts may be unavoidable. Reasons for this may be restrictions in choice caused by product specific cultivation requirements and the predominance of largely uncontrollable external factors in this phase. Despite the smaller contribution, processing and transportation stages may therefore still hold a larger improvement potential due to larger variations in the environmental profile of available technology and infrastructure in these stages. Long term measures to reduce the impact of the food system, relating to, for example, the development of new crop species and technological advancements as well as new production methods, should thus be targeted accordingly to maximise their potential. 


\section{Digesting the Pieces}

\section{Operational Aspects}

In contrast to the decision making on the strategic level, rethinking operational aspects generally allows to find solutions that can be implemented faster and have more immediate effects. While there are plenty of different operational aspects to consider in the food system, the focus in this research was on the last mile distribution of perishable products. Presenting the link between the underlying food production system and the end-consumers, the last mile covers a large variety of heterogeneous products, whereas other operational decisions are generally more product specific and thus may be less applicable to other products in the system.

The delivery of fresh and perishable products poses, moreover, a number of additional challenges to the already complex problem of last mile distribution. Integrated approaches, combining optimisation of routing and inventory decisions can help to reduce the overall costs associated with the last mile delivery in two-echelon distribution systems. While waste has not been explicitly addressed in this context, smooth inventory and distribution management can further help to avoid product spoilage and improve the final quality of products due to less time spent in transportation and storage phases.

In the context of last mile delivery, customer preferences may be modelled in different ways and can for example be incorporated in the form of alternative delivery patterns or tight time windows at the customer locations. In addition, customers may also opt for alternative delivery locations, in the form of customer pickup stations, allowing for more flexibility in terms of routing decisions and relaxing some of the time restrictions in the system. As a result, the implementation of such pickup stations for fresh products holds potential to reduce overall delivery costs despite the additional costs related to setup and refrigeration of these stations.

\subsubsection{Decisions - Consumption}

Consumption decisions generally relate to the demand in the system and determine our food and dietary choices, having a large impact on people's health and the environment. As a result, with non-communicable diseases and environmental concerns on the rise, more sustainable consumption choices that still ensure a healthy dietary intake are a highly relevant consideration in the current societal context. In connection with this, the research in this thesis investigated consumption choices on the large strategic scale of a country (in Chapters 2 and 3) as well as on a smaller individual scale, relating to more specific product concepts (in Chapter 6). In particular the shift towards plant-based diets has been explored in this relation and indicates the largest improvement potential for the different decision levels from an environmental perspective. 


\section{Network Perspective}

Investigating consumption decisions at the strategic level highlights the importance of incorporating supply chain and consumption decisions within a common framework, as consumption patterns largely affect the size and configuration of the underlying production system. Plant-based consumption is often associated with smaller systems, requiring less resource inputs and supply chain interactions, whereas meat-based consumption generally links to more complex systems with substantial resource requirements and many intermediary production steps. Looking at the replacement of beef and dairy consumption with more plant-based products, it is possible to make partial dietary replacements without a loss of key nutrients and reduce the associated environmental impact. Allowing for more drastic changes in the product mix, by only considering nutritional requirements of people rather than more specific demands for certain products, offers additional flexibility and holds a larger potential to reduce the overall environmental impact of our diet. It should however be noted that for the case of a fully plant-based consumption nutritional adequacy is only possible through vitamin B12 supplementation, as small amounts of animal products are needed otherwise to supply the required levels. Moreover, with respect to the impact of partial dietary changes on other nutrients the results indicate predominantly positive effects, allowing for healthy consumption alternatives in accordance with current dietary recommendations. Current consumer preferences and palatability constraints may however impact the acceptance of such dietary changes at the level of the individual consumer and thus require further consideration.

\section{Product Concepts}

The development of innovative product concepts, providing more sustainable alternatives to current food choices, can help to reduce the impact associated with our diet. Individual product replacements furthermore require smaller changes in consumer behaviour and may thus facilitate a shifting towards more plant-based consumption. Modelling the composition of a number of alternatives to beef and chicken shows, that it is possible to design products with equivalent profiles for the key nutrients, yet lower environmental impact values. Nutrient supplements/fortification may however be needed in some cases. When considering protein quality, soy presents a suitable ingredient due to its favourable amino-acid profile. In addition to plant-based ingredients, insects can also be used in meat alternatives to reduce the associated environmental impact, this holds in particular with regards to water use. The necessary processing steps for the production of such replacement products adds however to fossil fuel depletion and may thus lead to higher fossil fuel depletion values than for meat. 


\section{Digesting the Pieces}

\subsubsection{System Characteristics}

When analysing decisions in a system, it is important to take the characteristics of the respective system into account. Food poses particular challenges to planning and decision making due to the specific nature of products. Given the direct link to our health, consumption and supply of food need to meet certain nutritional, quality and safety requirements. The extensive range of products and activities involved as well as the interlinkages between individual aspects in the system further complicate the decision making process. In relation to this, the following will discuss the main system characteristics that have been addressed in this research and raise some of the aspects that require further investigations.

\section{Interlinkages}

The amount of product relations and interlinkages in the food system is vast, with livestock systems and compound products requiring extensive resource inputs from many different chains. Moreover, the food system is closely linked to other industries, valorising otherwise unused bi-products and side-streams. In addition to supply chain interrelations, relations may also exist between the consumption of certain products and nutrients that have been largely neglected in this research. Given this tremendous amount of interrelations, the scope of the overall system is difficult to define and requires decomposing the system into smaller decision problems that allow for easier analysis. Setting these system boundaries, however, affects the possible analysis and may result in unforeseen effects concerning other aspects of the system that lie outside these boundaries. Furthermore, in practice, relationships between products may not be inevitably fixed, but subject to change and uncertainty related to external factors that affect for instance the availability or quality of products. Results are therefore often highly dependent on the initial data input as well as the products, scenarios and subsystems under consideration. Despite these limitations, extending the scope and investigating links between certain aspects of the system allows decisions makers to better understand the system and the relationships within.

\section{Perishability}

Perishability and product decay are another common feature in the context of the food system, resulting in special handling requirements, related to food safety issues, and unnecessary product waste. Modelling perishability is however not always straight forward and depends on the individual product as well as a number of external factors, relating to for example temperature and other storage conditions. 
Product shelf-life presents a simplified way of depicting the duration of a product's lifespan, indicating the point in time that renders a product no longer adequate for consumption. The actual product lifespan, however, often exceeds product shelf-life due to a rather restrictive determination of the shelf-life in order to adhere to product safety standards and ensure a sufficient quality. This may result in products being discarded too early, thus leading to unnecessary value loss. Moreover, modelling perishability in the form of a $0-1$ decision, i.e. a product is either consumable or not, excludes cases where a product may still be partially consumed while other parts are being discarded. Considering a nutritional demand, products may for example still be perfectly safe to consume while their nutrient content for certain nutrients decreases over time. In other cases product lifespans can be extended through altered storage and/or processing decisions. For practical reasons, it is however still reasonable to assume specific product shelf-lives, despite these limitations.

Product decay functions in contrast present a more accurate way of depicting the quality of a product and thus its adequacy for consumption, by considering external factors in relation to the nature of the product. Models to estimate the deterioration of a product are generally product specific and relate to a certain type of deterioration, ranging from models using fixed-order kinetics, depicting nutrient and quality loss, to microbial growth models and methods using a Weibull distribution, describing sensory and chemical changes. In particular in situations where planning decisions concern many heterogeneous products with different shelf-lives or product decay functions it may therefore be interesting to investigate the effect of these differences. Moreover, while continuous decay functions pose challenges in terms of practical implementation, incorporating them in decision making models may shed light on the influence of external factors impacting the quality of a product in the system.

\section{Nutritional Requirements}

With human nutritional requirements being one of the underlying motivations for our consumption of food, nutritional aspects play an important role in the context of the food system. This research mostly considers nutrient requirements in the form of dietary reference values (DRVs) when evaluating dietary changes. Generally set at the national level, these DRVs however differ between countries, lacking a clear consent regarding ideal intake levels. Ideal intake levels further depend on the individual person as the response of the body to a particular diet may differ between people and depend on the level of activity carried out during a day. At the same time, the nutrient contents within foods may differ depending on for example ripeness or soil conditions, thus complicating the translation from DRVs to food based dietary guidelines. 


\section{Digesting the Pieces}

In addition, healthy diets and dietary quality are not determined exclusively by an adequate nutrient intake. Chewing processes, linked to the texture of consumed products, and aspects relating to volume and weight of the consumed diet, are for example influencing our digestive processes and feelings of satiation. It is therefore not recommendable, from a nutrition point of view, to provide a nutritionally feasible diet solely based on dietary supplementation and/or fortification. Furthermore, interactions between different components of food, can impede the absorption of nutrients within our body or encourage the bioavailability. Bioavailability is an important consideration requiring further attention, as iron from meat is for example better available than the same amount from plant foods, while vitamin $\mathrm{C}$ present in plant foods may in turn enhance the absorption of iron from plant foods.

Overall, it should be noted, that the full diet remains the best level to evaluate changes in our dietary consumption pattern, while the relationship between nutrient intakes and other dietary aspects requires more investigation. In the context of nutrient requirements, it is also important to mention that people generally make their choices on the food level rather than the nutrient level. Changes are thus mostly perceived on the food level, impacting the consumers' acceptance of certain changes. Food options and recommendations remain as such highly dependent on the cultural background of consumers, relating to different consumer preferences.

\subsubsection{Decision Makers}

Decision makers largely determine the perspective from which different choices are perceived and thereby define the decision environment. For the food system there are many different stakeholders and decision makers to consider, each with their own perspective and set of choices in the system. Instead of focusing on one perspective, this research investigates a rethinking of the system from the perspective of different decision makers in the system and thus allows for a more comprehensive view. In this context, the considered decision level impacts the range of decision makers and stakeholders relevant to the decision problem.

Decisions at the strategic level are mostly viewed in this thesis from the perspective of policy makers at the national level, though, other actors such as companies and individual consumers may have a stake in the decisions faced at the network level, as they are directly affected by the proposed change. Moreover, it should be noted that the research is conducted adopting the perspective of decision makers in the Netherlands, whereas decision makers in other countries will have other requirements, facing different objectives and constraints. The operational level in contrast is regarded from the perspective of decision makers at companies, operating in the area of last mile distribution. While the resulting 
decision problems are not necessarily specific to the Netherlands, the focus here mostly refers to last mile logistics in the developed world, due to specific infrastructure requirements. In contrast to strategic and operational decisions, decisions at the individual level refer to smaller decisions in the system, impacting only certain individual aspects. By investigating different product concepts, the research includes individual decision making at the industrial level.

Overall, the differences in perspective largely influence the objectives and preferences that have to be addressed and determine the nature of the decision problem at hand. The research shows also that due to the different needs of the actors there is not necessarily one problem common to all actors or one approach that fits all.

\subsubsection{Pressures on the System}

Depending on the perspective on the system, decision makers may be faced with different pressures in the system, resulting in a large range of impact indicators to choose from. While sustainability is mostly defined on the basis of economic, environmental and social aspects, the individual themes remain difficult to specify and largely depend on the considered decision problem. More generally, sustainability considerations can be seen as a sort of maintenance of the system, checking the functionality of its processes over time and replacing certain parts that no longer meet certain standards and requirements. As such, this research focuses on certain pressure points, that experience most strain in the system, in order to evaluate measures to maintain its functionality.

Investigating different performance indicators, the analysis shows that trade-offs exist between individual objectives, in particular in the context of environmental impact indicators. This has particular significance, as certain indicators have effects on a global scale, such as for example climate change, while others mostly impact the local level, such as land and water use. The choice of objective, therefore, plays an important role in the decision making process, affecting the configuration and impact contribution of the final solution. Multi-objective approaches can help to investigate these trade-offs and find a more balanced set of solutions, allowing decision makers to choose the best solution based on their preferences.

In addition to choosing meaningful objectives, difficulties related to sustainable decision making further include issues regarding the aggregation, measurement and quantification of certain impacts. This holds in particular for aspects linked to social sustainability, and gets further complicated on a global scale, where numerous social systems converge and interact. Altogether it can be concluded, that the pressures in the system as well as the resulting sustainability considerations remain a highly relevant multifaceted topic. 


\subsection{Anabolic Interactions - Synthesising Valuable Contributions}

Integrating the multiple different findings, this thesis makes a number of valuable contributions to the scientific literature and holds relevant insights for practical applications in the food system. This section elaborates on these contributions. In this context, it first reflects on the scientific contributions to the field of Operations Research, before shedding light on the applicability and societal relevance of this research.

\subsubsection{Contributions to the field of OR}

From an Operations Research perspective, this thesis presents a number of interesting application areas, introducing new decision problems that contribute to model building and the design of problem specific solution approaches. A brief overview of the range of problems covered in this thesis, as well as the nature of the proposed solution approaches is provided in Table 7.1.

Table 7.1: An overview of the Operations Research problems covered in this thesis

\begin{tabular}{|c|c|c|c|c|c|c|c|}
\hline \multirow{2}{*}{$\begin{array}{c}\text { Chapter } \\
\#\end{array}$} & \multicolumn{5}{|c|}{ Decision Problems } & \multicolumn{2}{|c|}{ Solution approach } \\
\hline & Network Flow & Inventory & Routing & Location & Blending & Exact & Heuristic \\
\hline 2 & $\checkmark$ & & & & $\checkmark$ & $\checkmark$ & \\
\hline 3 & $\checkmark$ & & & & $\checkmark$ & $\checkmark$ & \\
\hline 4 & & $\checkmark$ & $\checkmark$ & & & & $\checkmark$ \\
\hline 5 & & & $\checkmark$ & $\checkmark$ & & & $\checkmark$ \\
\hline 6 & & & & & $\checkmark$ & $\checkmark$ & \\
\hline
\end{tabular}

Spanning a broad range of decision problems, this thesis considers a more holistic approach to the rethinking of the food system and raises awareness of the potential of Operations Research approaches to support decision making in this area. As such, it contributes to the visibility of the field and promotes OR methods as tools for other scientific disciplines, such as the field of human nutrition or other food related sciences.

Within the field of OR, it links to a variety of well established research streams, including topics related to sustainable supply chain design, vehicle routing and dietary modelling. Building on the existing modelling approaches in these streams, it extends the scientific literature by adding new decision components as well as food specific characteristics to the considered decision environment. Furthermore, it attempts to underpin the practical relevance of such approaches through the use of real life data and relevant case studies. 
In this context, it also illustrates the potential of LCA data as input for models related to sustainability assessment in the food system while highlighting the need for more multiobjective approaches in this area.

Focusing on aspects related to modelling and solution approaches, the research in this thesis shows that even basic OR techniques can provide valuable insights into the pressing problems of today. At the same time, it indicates that increasingly sophisticated and datadriven approaches are needed to tackle in particular the more complex real world problems at the operational level.

\subsubsection{Added value for the field of Human Nutrition}

For nutritionists, this thesis provides valuable insights into issues related to the sustainability of our dietary choices. While sustainability considerations gain more attention, current nutrition research tends to focus solely on consumption aspects, therefore neglecting relationships between products and the link to the underlying supply chain configuration relating to the production and processing of products. As such, nutritional studies aimed at lowering the environmental impact of our diet miss important aspects that determine the true environmental impact of our diet. This results in dietary recommendations that are not always sensible or only partially valid from a food supply chain perspective. An example of such a recommendation is the proposed reduction of meat, and in particular beef, in combination with an increase in the consumption of dairy to compensate for some of the nutrients previously obtained from meat. The research in Chapters 2 and 3 of this thesis demonstrates the relevance of interrelations in the food system and illustrates possible implications of proposed dietary changes on the underlying system.

\subsubsection{Societal relevance and applicability}

With sustainability considerations and future food security gaining increasing attention in public discourse, demanding decision makers to take action and make better choices, this thesis addresses a highly relevant topic in today's society. As such, it provides insights into complex decision problems and raises further awareness with regards to the current pressures and challenges faced within the food system, thus contributing to the foundation that initialises change. The proposed models can be used as tools for analysis and decision support to facilitate decision makers at the level of policy makers and companies, allowing them to better understand the underlying problems, evaluate alternatives and implement change. The operational approaches related to the last mile distribution of products can furthermore be applied to plan the distribution of other perishable products outside of the food system, such as flowers or pharmaceutical products. 


\section{Digesting the Pieces}

Moreover, while some of the findings in this research are quite case specific due to the product specific data input used to illustrate the functionality of the models, the general applicability of the models transcends the presented cases and thus permits the investigation of other cases and scenarios that lie outside the scope of this research. The crucial issue in this context is the availability and accuracy of data with regards to the characteristics of the analysed part in the system. Despite these issues and limitations related to data, science relies on the often limited, available data as it provides the only way to derive general insights and facilitate analysis. Keeping this in mind, the issues related to the data used in this thesis have been highlighted in the individual chapters.

\subsection{Digestif - Tomorrow's Headache}

Several opportunities for future research directions arise from this thesis, affecting different aspects of the food system. The main directions are highlighted

\section{Decisions in the system}

- This thesis focuses on the strategic, operational and individual decision level within the food system, whereas decision levels are commonly structured according to strategic, tactical and operational aspects. Future research could therefore add to the research in this thesis by giving more attention to tactical decision making in the system.

- In addition to the decision problems considered in this thesis, other aspects in the food system relating to more product specific production and processing stages could be investigated. This holds in particular for the development of innovative concepts and technology targeted at reducing the environmental impact of the agricultural production phase.

- Extending the scope of this thesis, future research could investigate other relationships between products and supply chains in the food system and study connections between the food system and other industries.

\section{System Characteristics}

- This thesis mostly considers perishability in the form of fixed shelf-lives or linear deterioration of products. In practice these phenomena are however more complex with products deteriorating at different rates. Future research could thus investigate and exploit more refined modelling approaches related to the perishability of products. 
- Despite the predominance of uncertainty in our every day life, the research in this thesis focuses on deterministic modelling approaches. The food system faces uncertainty at all levels, affecting many different aspects related to the system and its characteristics. Future research directions could therefore be aimed at the development of modelling approaches that increasingly take the stochasticity and dynamic nature of problems into account.

- In addition to the system characteristics already considered in this research, future research could include other characteristics, intrinsic to the food system, such as the seasonality of products and the interactions production aspects with the natural environment.

\section{Decision Makers}

- The food system includes a large number of stakeholders and different decision makers that interact with each other on different levels of the system. This research neglects most of this interaction for reasons of simplicity, future research may however provide additional insights into the dynamics between decision makers in the food system by taking these interactions into account. Collaborative approaches between different decision makers could furthermore hold potential to reduce cost and the environmental impact associated with the food system.

- Consumer acceptance is one of the main criteria for the successful implementation of change. As such, future research should explore the acceptability of the proposed alternatives in this research from a consumer perspective and find ways to make alternatives more acceptable for the consumer.

\section{Pressures on the System}

- The amount of pressures on the system is large and versatile and future research may face new challenges that have to be addressed. Moreover, while this thesis already investigated several aspects related to sustainability, social aspects have received little attention in this context. Social considerations related to for example equity and other ethical aspects in the system play an important role in sustainability and hence require further investigation. From a modelling perspective, future research needs to find ways to meaningfully quantify, measure and aggregate these aspects.

- The findings of this thesis show, that trade-offs between indicators play an important role in the context of finding more sustainable solutions. Multi-criteria and multiobjective approaches hold potential to deal with these trade-offs and find more balanced solutions that take the decision makers preferences into account. Further investigation of these trade-offs as well as decision makers' preferences could benefit the analysis and help to come up with more meaningful alternatives in the future. 



\section{References}

Ahumada, O. and Villalobos, J. R. (2009). Application of planning models in the agri-food supply chain: A review. European Journal of Operational Research, 196(1):1-20.

Aiking, H. (2011). Future protein supply. Trends in Food Science $\&$ Technology, $22(2-3): 112-120$.

Akkerman, R., Farahani, P., and Grunow, M. (2010). Quality, safety and sustainability in food distribution: A review of quantitative operations management approaches and challenges. OR Spectrum, 32(4):863-904.

Albareda-Sambola, M. (2015). Location-routing and location-arc routing problems.

Laporte G., Nickel S., Saldanha da Gama F., eds. In Location science, pages 399-418. Springer, Berlin-Heidelberg.

Alder, J., Barling, D., Dugan, P., Herren, H. R., Josupeit, H., Lang, T., Lele, U., McClennen, C., Murphy-Bokern, D., Scherr, S., et al. (2012). Avoiding future famines: Strengthening the ecological foundation of food security through sustainable food systems. A UNEP Synthesis Report. Nairobi (Kenya): United Nations Environment Programme.

Allahyari, S., Salari, M., and Vigo, D. (2015). A hybrid metaheuristic algorithm for the multi-depot covering tour vehicle routing problem. European Journal of Operational Research, 242(3):756-768.

Allaoui, H., Guo, Y., Choudhary, A., and Bloemhof, J. (2018). Sustainable agro-food supply chain design using two-stage hybrid multi-objective decision-making approach. Computers 83 Operations Research, 89:369-384.

Amorim, P. and Almada-Lobo, B. (2014). The impact of food perishability issues in the vehicle routing problem. Computers \& Industrial Engineering, 67:223-233.

Amorim, P., Meyr, H., Almeder, C., and Almada-Lobo, B. (2013). Managing perishability in production-distribution planning: A discussion and review. Flexible Services and Manufacturing Journal, 25(3):389-413. 


\section{REFERENCES}

Amorim, P., Parragh, S. N., Sperandio, F., and Almada-Lobo, B. (2014). A rich vehicle routing problem dealing with perishable food: A case study. TOP, 22(2):489-508.

Andersson, H., Hoff, A., Christiansen, M., Hasle, G., and Løkketangen, A. (2010). Industrial aspects and literature survey: Combined inventory management and routing. Computers $\&$ Operations Research, 37(9):1515-1536.

Arima, E. Y., Richards, P., Walker, R., and Caldas, M. M. (2011). Statistical confirmation of indirect land use change in the brazilian amazon. Environmental Research Letters, 6(2):024010.

Arnold, F., Cardenas, I., Sörensen, K., and Dewulf, W. (2018). Simulation of B2C e-commerce distribution in Antwerp using cargo bikes and delivery points. European Transport Research Review, 10(1):2.

Asgar, M. A., Fazilah, A., Huda, N., Bhat, R., and Karim, A. A. (2010). Nonmeat protein alternatives as meat extenders and meat analogs. Comprehensive Reviews in Food Science and Food Safety, 9(5):513-529.

Ashby, A., Leat, M., and Hudson-Smith, M. (2012). Making connections: A review of supply chain management and sustainability literature. Supply Chain Management: An International Journal, 17(5):497-516.

Aubé, F. (2001). Guide for computing CO2 emissions, related to energy use. Natural Gas, 1(37):23.

Avadí, A., Fréon, P., and Quispe, I. (2014). Environmental assessment of Peruvian anchoveta food products: Is less refined better? The International Journal of Life Cycle Assessment, 19(6):1276-1293.

Averink, B. (2015). Vivera product information. Personal Communication.

Azadeh, A., Elahi, S., Farahani, M. H., and Nasirian, B. (2017). A genetic algorithm-taguchi based approach to inventory routing problem of a single perishable product with transshipment. Computers 63 Industrial Engineering, 104:124-133.

Azi, N., Gendreau, M., and Potvin, J.-Y. (2014). An adaptive large neighborhood search for a vehicle routing problem with multiple routes. Computers $\&$ Operations Research, 41:167-173.

Baldacci, R., Bartolini, E., and Laporte, G. (2010). Some applications of the generalized vehicle routing problem. Journal of the Operational Research Society, 61(7):1072-1077.

Baldacci, R. and Dell'Amico, M. (2010). Heuristic algorithms for the multi-depot ring-star problem. European Journal of Operational Research, 203(1):270-281.

Baldacci, R., Hill, A., Hoshino, E. A., and Lim, A. (2017). Pricing strategies for 
capacitated ring-star problems based on dynamic programming algorithms. European Journal of Operational Research, 262(3):879-893.

Banasik, A., Bloemhof-Ruwaard, J. M., Kanellopoulos, A., Claassen, G. D. H., and van der Vorst, J. G. A. J. (2018). Multi-criteria decision making approaches for green supply chains: A review. Flexible Services and Manufacturing Journal, 30(3):366-396.

Barona, E., Ramankutty, N., Hyman, G., and Coomes, O. T. (2010). The role of pasture and soybean in deforestation of the brazilian amazon. Environmental Research Letters, 5(2):024002.

Baroni, L., Cenci, L., Tettamanti, M., and Berati, M. (2007). Evaluating the environmental impact of various dietary patterns combined with different food production systems. European Journal of Clinical Nutrition, 61(2):279.

Bektas, T., Crainic, T., and van Woensel, T. (2017). From managing urban freight to smart city logistics networks Pardalos P., Gakis K., eds. In Network Design and Optimization for Smart Cities. World Scientific Publishing.

Bektaş, T., Erdoğan, G., and Røpke, S. (2011). Formulations and branch-and-cut algorithms for the generalized vehicle routing problem. Transportation Science, 45(3):299-316.

Bender, A. E. (1992). Meat and meat products in human nutrition in developing countries. FAO Rome.

Berners-Lee, M., Hoolohan, C., Cammack, H., and Hewitt, C. N. (2012). The relative greenhouse gas impacts of realistic dietary choices. Energy Policy, 43:184-190.

Bertazzi, L., Savelsbergh, M., and Speranza, M. G. (2008). Inventory routing. Golden B.L., Raghavan S, Wasil E.A., eds. In The vehicle routing problem: Latest advances and new challenges, pages 49-72. Springer, New York.

Beske, P., Land, A., and Seuring, S. (2014). Sustainable supply chain management practices and dynamic capabilities in the food industry: A critical analysis of the literature. International Journal of Production Economics, 152:131-143.

Billen, G., Lassaletta, L., and Garnier, J. (2014). A biogeochemical view of the global agro-food system: Nitrogen flows associated with protein production, consumption and trade. Global Food Security, 3(3-4):209-219.

Blonk, H., Alvarado, C., and De Schryver, A. (2007). Milieuanalyse vleesproducten. PRé Consultants.

Blonk, H., Kool, A., Luske, B., de Waart, S., and ten Pierick, E. (2008). Milieueffecten van nederlandse consumptie van eiwitrijke producten: Gevolgen van vervanging van dierlijke eiwitten anno 2008. Technical report, Blonk Milieu Advies BV, Gouda. 
Blonk Agri-footprint BV (2015a). Agri-footprint 2.0 - part 1 - methodology and basic principles. Gouda, the Netherlands.

Blonk Agri-footprint BV (2015b). Agri-footprint 2.0 - part 2 - description of data. Gouda, the Netherlands. retrieved from http://www .agri-footprint.com/methodology/methodology-report.html.

Bortolini, M., Faccio, M., Ferrari, E., Gamberi, M., and Pilati, F. (2016). Fresh food sustainable distribution: Cost, delivery time and carbon footprint three-objective optimization. Journal of Food Engineering, 174:56-67.

Boschetti, M. A., Maniezzo, V., Roffilli, M., and Röhler, A. B. (2009). Matheuristics: Optimization, simulation and control Blesa M.J., Blum C., Di Gaspero L., Roli A., Sampels M., Schaerf A., eds. In Hybrid Metaheuristics. HM 2009. Lecture Notes in Computer Science, volume 5818. Springer, Berlin, Heidelberg.

Boudoin, D., Morel, C., and Gardat, M. (2014). Supply chains and urban logistics platforms. Gonzalez-Feliu J., Semet F., Routhier J.-L., eds. In Sustainable urban logistics: Concepts, methods and information systems, pages 1-20. Springer, Berlin-Heidelberg.

Bozorgi, A., Pazour, J., and Nazzal, D. (2014). A new inventory model for cold items that considers costs and emissions. International Journal of Production Economics, 155:114-125.

Brandenburg, M., Govindan, K., Sarkis, J., and Seuring, S. (2014). Quantitative models for sustainable supply chain management: Developments and directions. European Journal of Operational Research, 233(2):299-312.

Brandenburg, M. and Rebs, T. (2015). Sustainable supply chain management: A modeling perspective. Annals of Operations Research, 229(1):213-252.

Broekema, R. and Blonk, H. (2009). Milieukundige vergelijking van vleesvervangers. Technical report, Blonk Milieu Advies BV, Gouda.

Broekema, R. and Smale, E. (2011). Nulmeting peulvruchten: Inzicht in milieueffecten en nutritionele aspecten van peulvruchten. Technical report, Blonk Milieu Advies BV, Gouda.

Bukkens, S. G. F. (1997). The nutritional value of edible insects. Ecology of Food and Nutrition, 36(2-4):287-319.

Carter, C. R. and Liane Easton, P. (2011). Sustainable supply chain management: Evolution and future directions. International Journal of Physical Distribution \&6 Logistics Management, 41(1):46-62.

Castanheira, É. G. and Freire, F. (2013). Greenhouse gas assessment of soybean 
production: Implications of land use change and different cultivation systems. Journal of Cleaner Production, 54:49-60.

Castellini, C., Boggia, A., Paolotti, L., Thoma, G. J., and Kim, D. (2012).

Environmental impacts and life cycle analysis of organic meat production and processing. Organic Meat Production and Processing, 53:1.

Cattaruzza, D., Absi, N., Feillet, D., and González-Feliu, J. (2017). Vehicle routing problems for city logistics. EURO Journal on Transportation and Logistics, $6(1): 51-79$.

Cerutti, A. K., Bruun, S., Beccaro, G. L., and Bounous, G. (2011). A review of studies applying environmental impact assessment methods on fruit production systems. Journal of Environmental Management, 92(10):2277-2286.

Clark, G. (2007). Evolution of the global sustainable consumption and production policy and the United Nations Environment Programme's (UNEP) supporting activities. Journal of Cleaner Production, 15(6):492-498.

Coe, M. T., Marthews, T. R., Costa, M. H., Galbraith, D. R., Greenglass, N. L., Imbuzeiro, H. M. A., Levine, N. M., Malhi, Y., Moorcroft, P. R., Muza, M. N., et al. (2013). Deforestation and climate feedbacks threaten the ecological integrity of south-southeastern Amazonia. Philosophical Transactions of the Royal Society B: Biological Sciences, 368(1619):20120155.

Coelho, L. C., Cordeau, J.-F., and Laporte, G. (2013). Thirty years of inventory routing. Transportation Science, 48(1):1-19.

Coelho, L. C. and Laporte, G. (2014). Optimal joint replenishment, delivery and inventory management policies for perishable products. Computers $\&$ Operations Research, 47:42-52.

Contardo, C., Hemmelmayr, V., and Crainic, T. G. (2012). Lower and upper bounds for the two-echelon capacitated location-routing problem. Computers $\&$ Operations Research, 39(12):3185-3199.

Cordeau, J.-F., Desaulniers, G., Desrosiers, J., Solomon, M., and Soumis, F. (2002). VRP with time windows. Toth, P., Vigo, D., eds. In The Vehicle Routing Problem, pages 157-194 (Chapter 7). SIAM Monographs on Discrete Mathematics and Applications, Philadeplhia.

Cordeau, J.-F., Gendreau, M., and Laporte, G. (1997). A tabu search heuristic for periodic and multi-depot vehicle routing problems. Networks: An International Journal, 30(2):105-119.

Cordeau, J.-F., Laporte, G., Savelsbergh, M. W., and Vigo, D. (2007). Vehicle routing. Handbooks in Operations Research and Management Science, 14:367-428. 
Costa, M. H., Yanagi, S. N. M., Souza, P. J. O. P., Ribeiro, A., and Rocha, E. J. P. (2007). Climate change in amazonia caused by soybean cropland expansion, as compared to caused by pastureland expansion. Geophysical Research Letters, 34(7).

Crainic, T. G., Ricciardi, N., and Storchi, G. (2009). Models for evaluating and planning city logistics systems. Transportation Science, 43(4):432-454.

Crainic, T. G., Sforza, A., and Sterle, C. (2011). Location-routing models for two-echelon freight distribution system design. Technical report, CIRRELT-2011-40. CIRRELT Montréal.

Cuda, R., Guastaroba, G., and Speranza, M. G. (2015). A survey on two-echelon routing problems. Computers $\&$ Operations Research, 55:185-199.

Da Silva, V. P., van der Werf, H. M. G., Spies, A., and Soares, S. R. (2010). Variability in environmental impacts of brazilian soybean according to crop production and transport scenarios. Journal of Environmental Management, 91(9):1831-1839.

Dani, S. (2015). Food supply chain management and logistics: From farm to fork. Kogan Page Publishers, London.

Davis, J., Sonesson, U., Baumgartner, D. U., and Nemecek, T. (2010). Environmental impact of four meals with different protein sources: Case studies in Spain and Sweden. Food Research International, 43(7):1874-1884.

Davis, T. A., Nguyen, H. V., Garcia-Bravo, R., Fiorotto, M. L., Jackson, E. M., and Reeds, P. J. (1994). Amino acid composition of the milk of some mammalian species changes with stage of lactation. British Journal of Nutrition, 72(6):845-853.

Day, L. (2013). Proteins from land plants-potential resources for human nutrition and food security. Trends in Food Science \& Technology, 32(1):25-42.

de Vries, M. and de Boer, I. J. M. (2010). Comparing environmental impacts for livestock products: A review of life cycle assessments. Livestock Science, 128(1-3):1-11.

Dekker, R., Bloemhof, J., and Mallidis, I. (2012). Operations research for green logistics - an overview of aspects, issues, contributions and challenges. European Journal of Operational Research, 219(3):671-679.

Diabat, A., Abdallah, T., and Le, T. (2016). A hybrid tabu search based heuristic for the periodic distribution inventory problem with perishable goods. Annals of Operations Research, 242(2):373-398.

Drexl, M. and Schneider, M. (2015). A survey of variants and extensions of the location-routing problem. European Journal of Operational Research, 241(2):283-308.

Ehrgott, M. (2009). Multicriteria optimization. Springer Science \& Business Media, 
Berlin-Heidelberg.

Eskandarpour, M., Dejax, P., Miemczyk, J., and Péton, O. (2015). Sustainable supply chain network design: An optimization-oriented review. Omega, 54:11-32.

FAO (2004). Protein sources for the animal feed industry. Expert consultation and workshop. Bangkok, 29 april-3 may 2002.

FAO (2009). Global agriculture towards 2050. FAO high-level expert forum, Rome.

FAO (2011). Global food losses and food waste: Extent, causes and prevention.

FAO (2017). The future of food and agriculture-trends and challenges.

FAO (2018). Sustainable food systems - concept and framework.

FAO/INFOODS (2013). FAO/INFOODS food composition database for biodiversity version 2.1-biofoodcomp2. 1. FAO Rome.

FAO/WHO (2002). Human vitamin and mineral requirements. World Health Organisation, Thailand (2002).

FAO/WHO/UNU (2002). Protein and amino acid requirements in human nutrition WHO technical report series, vol. 935. WHO, Geneva, Switzerland (2002).

Farahani, P., Grunow, M., and Günther, H.-O. (2012). Integrated production and distribution planning for perishable food products. Flexible Services and Manufacturing Journal, 24(1):28-51.

Fearnside, P. M. (2018). Brazil's amazonian forest carbon: The key to Southern Amazonia's significance for global climate. Regional Environmental Change, 18(1):47-61.

Fiala, N. (2008). Meeting the demand: An estimation of potential future greenhouse gas emissions from meat production. Ecological Economics, 67(3):412-419.

Finnveden, G., Hauschild, M. Z., Ekvall, T., Guinée, J., Heijungs, R., Hellweg, S., Koehler, A., Pennington, D., and Suh, S. (2009). Recent developments in life cycle assessment. Journal of Environmental Management, 91(1):1-21.

Flindall (2016). Vitaminen van flindall. retrieved on 06.04.2016, from https://www.flinndal.nl/.

Flores-Garza, D. A., Salazar-Aguilar, M. A., Ngueveu, S. U., and Laporte, G. (2017). The multi-vehicle cumulative covering tour problem. Annals of Operations Research, 258(2):761-780.

Foster, C., Guében, C., Holmes, M., Wiltshire, J., and Wynn, S. (2014). The environmental effects of seasonal food purchase: A raspberry case study. Journal of Cleaner Production, 73:269-274. 
Friedman, M. (1996). Nutritional value of proteins from different food sources: A review. Journal of Agricultural and Food Chemistry, 44(1):6-29.

Garnett, T. (2011). Where are the best opportunities for reducing greenhouse gas emissions in the food system (including the food chain)? Food Policy, 36:S23-S32.

Garnett, T. (2013). Food sustainability: Problems, perspectives and solutions. Proceedings of the Nutrition Society, 72(1):29-39.

Gasparri, N. I., Grau, H. R., and Angonese, J. G. (2013). Linkages between soybean and neotropical deforestation: Coupling and transient decoupling dynamics in a multi-decadal analysis. Global Environmental Change, 23(6):1605-1614.

Gevaers, R., Van de Voorde, E., and Vanelslander, T. (2009). Characteristics of innovations in last-mile logistics-using best practices, case studies and making the link with green and sustainable logistics. In Proceedings of the European transport conference (2009). Association for European Transport and Contributors, London.

Goedkoop, M., Heijungs, R., De Schryver, A., Struijs, J., and Van Zelm, R. (2009). Recipe 2008: A life cycle impact assessment method which comprises harmonised category indicators at the midpoint and the endpoint level. Technical report, RIVM report. Bilthoven, Netherlands: RIVM.

Goedkoop, M., Heijungs, R., De Schryver, A., Struijs, J., and Van Zelm, R. (2013). Recipe 2008: A life cycle impact assessment method which comprises harmonised category indicators at the midpoint and the endpoint level - characterisation. Technical report, Updated RIVM report. Bilthoven, Netherlands: RIVM.

Govindan, K., Jafarian, A., Khodaverdi, R., and Devika, K. (2014). Two-echelon multiple-vehicle location-routing problem with time windows for optimization of sustainable supply chain network of perishable food. International Journal of Production Economics, 152:9-28.

Ha, M. H., Bostel, N., Langevin, A., and Rousseau, L.-M. (2013). An exact algorithm and a metaheuristic for the multi-vehicle covering tour problem with a constraint on the number of vertices. European Journal of Operational Research, 226(2):211-220.

Ha, M. H., Bostel, N., Langevin, A., and Rousseau, L.-M. (2014). An exact algorithm and a metaheuristic for the generalized vehicle routing problem with flexible fleet size. Computers \& Operations Research, 43:9-19.

Hallström, E., Carlsson-Kanyama, A., and Börjesson, P. (2015). Environmental impact of dietary change: a systematic review. Journal of Cleaner Production, 91:1-11.

Hassini, E., Surti, C., and Searcy, C. (2012). A literature review and a case study of sustainable supply chains with a focus on metrics. International Journal of Production Economics, 140(1):69-82. 
Hauschild, M. Z., Goedkoop, M., Guinée, J., Heijungs, R., Huijbregts, M., Jolliet, O., Margni, M., De Schryver, A., Humbert, S., Laurent, A., et al. (2013). Identifying best existing practice for characterization modeling in life cycle impact assessment. The International Journal of Life Cycle Assessment, 18(3):683-697.

Hayashi, K. (2000). Multicriteria analysis for agricultural resource management: a critical survey and future perspectives. European Journal of Operational Research, $122(2): 486-500$.

Health Council of the Netherlands (2011). Guidelines for a healthy diet: The ecological perspective. Health Council of the Netherlands, The Hague.

Hemmelmayr, V. C., Cordeau, J.-F., and Crainic, T. G. (2012). An adaptive large neighborhood search heuristic for two-echelon vehicle routing problems arising in city logistics. Computers $\&$ Operations Research, 39(12):3215-3228.

Hendriks, T. H. B. (2013). Duality and sensitivity analysis. In Decision Sciences, Theory and Applications, pages 95-116. Wageningen Academic Publishers.

Hiassat, A. and Diabat, A. (2011). A location inventory routing problem with perishable products. In Proceedings of the 41st International Conference on Computers and Industrial Engineering, pages 130-135. Curran Associates, Inc., Los Angeles.

Hiassat, A., Diabat, A., and Rahwan, I. (2017). A genetic algorithm approach for location-inventory-routing problem with perishable products. Journal of Manufacturing Systems, 42:93-103.

Higgins, A. J., Miller, C. J., Archer, A. A., Ton, T., Fletcher, C. S., and McAllister, R. R. J. (2010). Challenges of operations research practice in agricultural value chains. Journal of the Operational Research Society, 61(6):964-973.

Hill, A. and Voß, S. (2016). An equi-model matheuristic for the multi-depot ring star problem. Networks, 67(3):222-237.

Hsu, C.-I., Hung, S.-F., and Li, H.-C. (2007). Vehicle routing problem with time-windows for perishable food delivery. Journal of Food Engineering, 80(2):465-475.

Iakovou, E., Bochtis, D., Vlachos, D., and Aidonis, D. (2016). Supply chain management for sustainable food networks. John Wiley \& Sons, Chichester.

Ingram, J. (2011). A food systems approach to researching food security and its interactions with global environmental change. Food Security, 3(4):417-431.

Ingwersen, W. W. (2012). Life cycle assessment of fresh pineapple from Costa Rica. Journal of Cleaner Production, 35:152-163.

Jaehn, F. (2016). Sustainable operations. European Journal of Operational Research, 
253(2):243-264.

Jia, T., Li, X., Wang, N., and Li, R. (2014). Integrated inventory routing problem with quality time windows and loading cost for deteriorating items under discrete time. Mathematical Problems in Engineering, 2014.

Jozefowiez, N. (2014). A branch-and-price algorithm for the multivehicle covering tour problem. Networks, 64(3):160-168.

Kande, S., Prins, C., Belgacem, L., and Redon, B. (2015). Local search based metaheuristics for two-echelon distribution network with perishable products. In International Conference on Operations Research and Enterprise Systems, pages 212-231. Springer, Cham.

Karaoğlan, İ., Erdoğan, G., and Koç, Ç. (2018). The multi-vehicle probabilistic covering tour problem. European Journal of Operational Research, 271(1):278-287.

Koç, C.., Bektaş, T., Jabali, O., and Laporte, G. (2016). The impact of depot location, fleet composition and routing on emissions in city logistics. Transportation Research Part B: Methodological, 84:81-102.

Krintiras, G. A., Diaz, J. G., Van Der Goot, A. J., Stankiewicz, A. I., and Stefanidis, G. D. (2016). On the use of the couette cell technology for large scale production of textured soy-based meat replacers. Journal of Food Engineering, 169:205-213.

Laporte, G. (2009). Fifty years of vehicle routing. Transportation Science, 43(4):408-416.

Le, T., Diabat, A., Richard, J.-P., and Yih, Y. (2013). A column generation-based heuristic algorithm for an inventory routing problem with perishable goods. Optimization Letters, 7(7):1481-1502.

Li, Y., Chu, F., Yang, Z., and Calvo, R. W. (2016). A production inventory routing planning for perishable food with quality consideration. IFAC-PapersOnLine, 49(3):407-412.

Lima, M., Skutsch, M., and Costa, G. (2011). Deforestation and the social impacts of soy for biodiesel: Perspectives of farmers in the South Brazilian Amazon. Ecology and Society, 16(4).

Linnemann, A. R., Hendrix, E. M. T., Apaiah, R., and van Boekel, T. A. J. S. (2015). Food chain design using multi criteria decision making, an approach to complex design issues. NJAS-Wageningen Journal of Life Sciences, 72:13-21.

MacDiarmid, J. I., Kyle, J., Horgan, G. W., Loe, J., Fyfe, C., Johnstone, A., and McNeill, G. (2012). Sustainable diets for the future: Can we contribute to reducing greenhouse gas emissions by eating a healthy diet? The American Journal of Clinical 
Nutrition, 96(3):632-639.

Macedo, M. N., DeFries, R. S., Morton, D. C., Stickler, C. M., Galford, G. L., and Shimabukuro, Y. E. (2012). Decoupling of deforestation and soy production in the southern amazon during the late 2000s. Proceedings of the National Academy of Sciences, 109(4):1341-1346.

Maguire, C., Belchior, C., Hoogeveen, Y., Westhoek, H., and Manshoven, S. (2017). Food in a green light - a systems approach to sustainable food. European Environment Agency.

Mallidis, I., Dekker, R., and Vlachos, D. (2012). The impact of greening on supply chain design and cost: A case for a developing region. Journal of Transport Geography, 22:118-128.

Mancini, S., Gonzalez-Feliu, J., and Crainic, T. G. (2014). Planning and optimization methods for advanced urban logistics systems at tactical level. Gonzalez-Feliu J., Semet F., Routhier J.-L., eds. In Sustainable Urban Logistics: Concepts, Methods and Information Systems, pages 145-164. Springer, Berlin-Heidelberg.

Margni, M. and Curran, M. A. (2012). Life cycle impact assessment. Life cycle assessment handbook: A guide for Environmentally Sustainable Products, pages 67-103.

Meier, M. S., Stoessel, F., Jungbluth, N., Juraske, R., Schader, C., and Stolze, M. (2015). Environmental impacts of organic and conventional agricultural products are the differences captured by life cycle assessment? Journal of Environmental Management, 149:193-208.

Meier, T. and Christen, O. (2012). Environmental impacts of dietary recommendations and dietary styles: Germany as an example. Environmental Science $\&$ Technology, 47(2):877-888.

Mekonnen, M. M. and Hoekstra, A. Y. (2011). The green, blue and grey water footprint of crops and derived crop products. Hydrology and Earth System Sciences, 15(5):1577-1600.

Mekonnen, M. M. and Hoekstra, A. Y. (2013). A global assessment of the water footprint of farm animal products. Ecosystems, 15(3):401-415.

Mertens, E., van’t Veer, P., Hiddink, G. J., Steijns, J. M. J. M., and Kuijsten, A. (2017). Operationalising the health aspects of sustainable diets: A review. Public Health Nutrition, 20(4):739-757.

Mirzaei, S. and Seifi, A. (2015). Considering lost sale in inventory routing problems for perishable goods. Computers \& Industrial Engineering, 87:213-227. 
Mishra, N., El-Aal Bakr, A. A., Niranjan, K., and Tucker, G. (2012). Environmental aspects of food processing. Food processing handbook, pages 571-591.

Mogensen, L., Hermansen, J. E., Halberg, N., Dalgaard, R., Vis, J. C., and Smith, B. G. (2009). Life cycle assessment across the food supply chain. Sustainability in the Food Industry, pages 115-144.

Mollenhorst, H., Berentsen, P. B. M., and De Boer, I. J. M. (2006). On-farm quantification of sustainability indicators: An application to egg production systems. British Poultry Science, 47(4):405-417.

Mollenkopf, D., Stolze, H., Tate, W. L., and Ueltschy, M. (2010). Green, lean, and global supply chains. International Journal of Physical Distribution 83 Logistics Management, 40(1/2):14-41.

Monsen, E. R., Hallberg, L., Layrisse, M., Hegsted, D. M., Cook, J., Mertz, W., and Finch, C. A. (1978). Estimation of available dietary iron. The American Journal of Clinical Nutrition, 31(1):134-141.

Morganti, E., Seidel, S., Blanquart, C., Dablanc, L., and Lenz, B. (2014). The impact of e-commerce on final deliveries: Alternative parcel delivery services in France and Germany. Transportation Research Procedia, 4:178-190.

Nagurney, A. and Nagurney, L. S. (2010). Sustainable supply chain network design: A multicriteria perspective. International Journal of Sustainable Engineering, 3(3):189-197.

Nagy, G. and Salhi, S. (2007). Location-routing: Issues, models and methods. European Journal of Operational Research, 177(2):649-672.

Nahmias, S. (2011). Perishable inventory systems, volume 160. Springer Science \& Business Media, New York.

Nedjati, A., Izbirak, G., and Arkat, J. (2017). Bi-objective covering tour location routing problem with replenishment at intermediate depots: Formulation and meta-heuristics. Computers \& Industrial Engineering, 110:191-206.

Nguyen, V.-P., Prins, C., and Prodhon, C. (2012). Solving the two-echelon location routing problem by a grasp reinforced by a learning process and path relinking. European Journal of Operational Research, 216(1):113-126.

Nijdam, D., Rood, T., and Westhoek, H. (2012). The price of protein: Review of land use and carbon footprints from life cycle assessments of animal food products and their substitutes. Food Policy, 37(6):760-770.

Notarnicola, B., Hayashi, K., Curran, M. A., and Huisingh, D. (2012). Progress in working towards a more sustainable agri-food industry. Journal of Cleaner 
Production, 28:1-8.

Notarnicola, B., Tassielli, G., Renzulli, P. A., Castellani, V., and Sala, S. (2017). Environmental impacts of food consumption in Europe. Journal of Cleaner Production, 140:753-765.

Oglethorpe, D. (2010). Optimising economic, environmental, and social objectives: A goal-programming approach in the food sector. Environment and Planning A, 42(5):1239-1254.

Oonincx, D. G. A. B. and De Boer, I. J. M. (2012). Environmental impact of the production of mealworms as a protein source for humans - a life cycle assessment. PloS one, 7(12):e51145.

Osvald, A. and Stirn, L. Z. (2008). A vehicle routing algorithm for the distribution of fresh vegetables and similar perishable food. Journal of Food Engineering, 85(2):285-295.

Pardo, G. and Zufía, J. (2012). Life cycle assessment of food-preservation technologies. Journal of Cleaner Production, 28:198-207.

Parfitt, J., Barthel, M., and Macnaughton, S. (2010). Food waste within food supply chains: Quantification and potential for change to 2050. Philosophical Transactions of the Royal Society B: Biological Sciences, 365(1554):3065-3081.

Persson, U. M., Henders, S., and Cederberg, C. (2014). A method for calculating a land-use change carbon footprint (LUC-CFP) for agricultural commodities applications to Brazilian beef and soy, Indonesian palm oil. Global Change Biology, 20(11):3482-3491.

Pham, T. A., Hà, M. H., and Nguyen, X. H. (2017). Solving the multi-vehicle multi-covering tour problem. Computers $\&$ Operations Research, 88:258-278.

Pop, P. C., Kara, I., and Marc, A. H. (2012). New mathematical models of the generalized vehicle routing problem and extensions. Applied Mathematical Modelling, 36(1):97-107.

Prodhon, C. and Prins, C. (2014). A survey of recent research on location-routing problems. European Journal of Operational Research, 238(1):1-17.

Rabbani, M., Farshbaf-Geranmayeh, A., and Haghjoo, N. (2016). Vehicle routing problem with considering multi-middle depots for perishable food delivery. Uncertain Supply Chain Management, 4(3):171-182.

Rabbani, M., Ramezankhani, M. J., Farrokhi-Asl, H., and Farshbaf-Geranmayeh, A. (2015). Vehicle routing with time windows and customer selection for perishable goods. International Journal of Supply and Operations Management, 2(2):700. 
Rahimi, M., Baboli, A., and Rekik, Y. (2017). Inventory routing problem for perishable products by considering customer satisfaction and green criteria. Freitag M., Kotzab H., Pannek J. (eds). In Dynamics in Logistics, pages 445-455. Springer, Cham.

Ribal, J., Fenollosa, M. L., García-Segovia, P., Clemente, G., Escobar, N., and Sanjuán, N. (2016). Designing healthy, climate friendly and affordable school lunches. The International Journal of Life Cycle Assessment, 21(5):631-645.

RIVM (2013). Nederlands voedingsstoffenbestand online database.

Romero, C. (2001). Extended lexicographic goal programming: A unifying approach. Omega, 29(1):63-71.

Romero, C. and Rehmann, T. (2003). Chapter five compromise programming developments in agricultural economics. Elsevier, 11:63-78.

Rong, A., Akkerman, R., and Grunow, M. (2011). An optimization approach for managing fresh food quality throughout the supply chain. International Journal of Production Economics, 131(1):421-429.

Ropke, S. and Pisinger, D. (2006). An adaptive large neighborhood search heuristic for the pickup and delivery problem with time windows. Transportation Science, 40(4):455-472.

Sabaté, J. and Soret, S. (2014). Sustainability of plant-based diets: back to the future. The American Journal of Clinical Nutrition, 100(suppl_1):476S-482S.

Sampaio, G., Nobre, C., Costa, M. H., Satyamurty, P., Soares-Filho, B. S., and Cardoso, M. (2007). Regional climate change over eastern Amazonia caused by pasture and soybean cropland expansion. Geophysical Research Letters, 34(17).

Sasu-Boakye, Y., Cederberg, C., and Wirsenius, S. (2014). Localising livestock protein feed production and the impact on land use and greenhouse gas emissions. Animal, 8(8):1339-1348.

Sauer, B. (2012). Life cycle inventory modeling in practice. Life Cycle Assessment Handbook: A Guide for Environmentally Sustainable Products, pages 43-66.

Savelsbergh, M. and Van Woensel, T. (2016). 50th anniversary invited article city logistics: Challenges and opportunities. Transportation Science, 50(2):579-590.

Schlemmer, U., Frølich, W., Prieto, R. M., and Grases, F. (2009). Phytate in foods and significance for humans: Food sources, intake, processing, bioavailability, protective role and analysis. Molecular Nutrition 8 Food Research, 53(S2):S330-S375.

Schmid, V., Doerner, K. F., and Laporte, G. (2013). Rich routing problems arising in supply chain management. European Journal of Operational Research, 224(3):435-448.

Schösler, H., De Boer, J., and Boersema, J. J. (2012). Can we cut out the meat of the 
dish? Constructing consumer-oriented pathways towards meat substitution. Appetite, 58(1):39-47.

Schwengerer, M., Pirkwieser, S., and Raidl, G. R. (2012). A variable neighborhood search approach for the two-echelon location-routing problem. In European Conference on Evolutionary Computation in Combinatorial Optimization, pages 13-24. Springer, Berlin, Heidelberg.

Seuring, S. (2013). A review of modeling approaches for sustainable supply chain management. Decision Support Systems, 54(4):1513-1520.

Seuring, S. and Müller, M. (2008). From a literature review to a conceptual framework for sustainable supply chain management. Journal of Cleaner Production, 16(15):1699-1710.

Shaw, P. (1998). Using constraint programming and local search methods to solve vehicle routing problems. In International Conference on Principles and Practice of Constraint Programming, pages 417-431. Springer, Berlin-Heidelberg.

Sim, S., Barry, M., Clift, R., and Cowell, S. J. (2007). The relative importance of transport in determining an appropriate sustainability strategy for food sourcing. The International Journal of Life Cycle Assessment, 12(6):422.

Smetana, S., Mathys, A., Knoch, A., and Heinz, V. (2015). Meat alternatives: Life cycle assessment of most known meat substitutes. The International Journal of Life Cycle Assessment, 20(9):1254-1267.

Smith, B. G. (2007). Developing sustainable food supply chains. Philosophical Transactions of the Royal Society B: Biological Sciences, 363(1492):849-861.

Solomon, M. M. (1987). Algorithms for the vehicle routing and scheduling problems with time window constraints. Operations Research, 35(2):254-265.

Sonesson, U., Davis, J., Flysjö, A., Gustavsson, J., and Witthöft, C. (2017). Protein quality as functional unit - a methodological framework for inclusion in life cycle assessment of food. Journal of Cleaner Production, 140:470-478.

Sonesson, U., Mattsson, B., Nybrant, T., and Ohlsson, T. (2005). Industrial processing versus home cooking: An environmental comparison between three ways to prepare a meal. AMBIO: A Journal of the Human Environment, 34(4):414-422.

Song, B. D. and Ko, Y. D. (2016). A vehicle routing problem of both refrigerated-and general-type vehicles for perishable food products delivery. Journal of Food Engineering, 169:61-71.

Soysal, M., Bloemhof-Ruwaard, J. M., Haijema, R., and van der Vorst, J. G. A. J. (2015). Modeling an inventory routing problem for perishable products with 
environmental considerations and demand uncertainty. International Journal of Production Economics, 164:118-133.

Soysal, M., Bloemhof-Ruwaard, J. M., Meuwissen, M. P. M., and van der Vorst, J. G. A. J. (2012). A review on quantitative models for sustainable food logistics management. International Journal on Food System Dynamics, 3(2):136-155.

Soysal, M., Bloemhof-Ruwaard, J. M., and Van der Vorst, J. G. A. J. (2014). Modelling food logistics networks with emission considerations: The case of an international beef supply chain. International Journal of Production Economics, 152:57-70.

Srivastava, S. K. (2007). Green supply-chain management: A state-of-the-art literature review. International Journal of Management Reviews, 9(1):53-80.

Steinfeld, H., Gerber, P., Wassenaar, T. D., Castel, V., Rosales, M., Rosales, M., and de Haan, C. (2006). Livestock's long shadow: environmental issues and options. Food \& Agriculture Organization of the United Nations, Rome.

Sterle, C. (2010). Location-routing models and methods for freight distribution and infomobility in city logistics. Technical report, CIRRELT-2010-38. CIRRELT Montréal.

Tang, C. S. and Zhou, S. (2012). Research advances in environmentally and socially sustainable operations. European Journal of Operational Research, 223(3):585-594.

Tarantilis, C. and Kiranoudis, C. (2001). A meta-heuristic algorithm for the efficient distribution of perishable foods. Journal of Food Engineering, 50(1):1-9.

Temme, E. H., Van Der Voet, H., Thissen, J. T. N. M., Verkaik-Kloosterman, J., van Donkersgoed, G., and Nonhebel, S. (2013). Replacement of meat and dairy by plant-derived foods: Estimated effects on land use, iron and SFA intakes in young Dutch adult females. Public Health Nutrition, 16(10):1900-1907.

Toth, P. and Vigo, D. (2014). Vehicle routing: Problems, methods, and applications. SIAM, Philadelphia.

Trienekens, J. H., Wognum, P. M., Beulens, A. J. M., and van der Vorst, J. G. A. J. (2012). Transparency in complex dynamic food supply chains. Advanced Engineering Informatics, 26(1):55-65.

Tsolakis, N. K., Keramydas, C. A., Toka, A. K., Aidonis, D. A., and Iakovou, E. T. (2014). Agrifood supply chain management: A comprehensive hierarchical decision-making framework and a critical taxonomy. Biosystems Engineering, 120:47-64.

Tukker, A. and Jansen, B. (2006). Environmental impacts of products: A detailed review of studies. Journal of Industrial Ecology, 10(3):159-182. 
Tyszler, M., Kramer, G., and Blonk, H. (2014). Comparing apples with oranges: On the functional equivalence of food products for comparative LCAs. The International Journal of Life Cycle Assessment, 19(8):1482-1487.

Tyszler, M., Kramer, G., and Blonk, H. (2016). Just eating healthier is not enough: Studying the environmental impact of different diet scenarios for Dutch women (31-50 years old) by linear programming. The International Journal of Life Cycle Assessment, 21(5):701-709.

UN (2015). Resolution A/RES/70/1. Transforming our world: The 2030 agenda for sustainable development. Seventieth United Nations General Assembly, New York.

UNEP (2016). Food systems and natural resources. A Report of the Working Group on Food Systems of the International Resource Panel. Hajer, M A and Westhoek, Henk and Ingram, J and van Berkum, S and Özay, L.

USDA (2016). National nutrient database for standard reference release 28. retrieved 10.03.2016, from United States Department of Agriculture https://ndb.nal.usda.gov/ndb/foods.

Validi, S., Bhattacharya, A., and Byrne, P. J. (2014). A case analysis of a sustainable food supply chain distribution system - a multi-objective approach. International Journal of Production Economics, 152:71-87.

Van Der Vorst, J. G. A. J., Tromp, S.-O., and van der Zee, D.-J. (2009). Simulation modelling for food supply chain redesign; integrated decision making on product quality, sustainability and logistics. International Journal of Production Research, 47(23):6611-6631.

van Dooren, C., Marinussen, M., Blonk, H., Aiking, H., and Vellinga, P. (2014).

Exploring dietary guidelines based on ecological and nutritional values: A comparison of six dietary patterns. Food Policy, 44:36-46.

Van Kernebeek, H. R. J., Oosting, S. J., Feskens, E. J. M., Gerber, P. J., and De Boer, I. J. M. (2014). The effect of nutritional quality on comparing environmental impacts of human diets. Journal of Cleaner Production, 73:88-99.

Van Kernebeek, H. R. J., Oosting, S. J., Van Ittersum, M. K., Bikker, P., and De Boer, I. J. M. (2016). Saving land to feed a growing population: Consequences for consumption of crop and livestock products. The International Journal of Life Cycle Assessment, 21(5):677-687.

Van Middelaar, C. E., Berentsen, P. B. M., Dolman, M. A., and De Boer, I. J. M. (2011). Eco-efficiency in the production chain of Dutch semi-hard cheese. Livestock Science, 139(1-2):91-99.

Van Mierlo, K., Rohmer, S., and Gerdessen, J. C. (2017). A model for composing meat 
replacers: Reducing the environmental impact of our food consumption pattern while retaining its nutritional value. Journal of Cleaner Production, 165:930-950.

Van Rossum, C. T. M., Buurma-Rethans, E. J. M., Vennemann, F. B. C., Beukers, M., Brants, H. A. M., De Boer, E. J., and Ocké, M. C. (2016). The diet of the Dutch: Results of the first two years of the Dutch National Food Consumption Survey 2012-2016. RIVM letter report 2016-0082.

Verkerk, R., Schreiner, M., Krumbein, A., Ciska, E., Holst, B., Rowland, I., De Schrijver, R., Hansen, M., Gerhäuser, C., Mithen, R., et al. (2009). Glucosinolates in brassica vegetables: The influence of the food supply chain on intake, bioavailability and human health. Molecular Nutrition \& Food Research, 53(S2):S219-S219.

Vermeulen, S. J., Campbell, B. M., and Ingram, J. S. I. (2012). Climate change and food systems. Annual Review of Environment and Resources, 37:195-222.

Viglizzo, E. F., Frank, F. C., CarreÑo, L. V., Jobbagy, E. G., Pereyra, H., Clatt, J., Pincen, D., and Ricard, M. F. (2011). Ecological and environmental footprint of 50 years of agricultural expansion in Argentina. Global Change Biology, 17(2):959-973.

Vivera (2016). The vivera product range. retrieved on 10.03.2016, from http://www. vivera.com/en/home.

Voedingscentrum (2016). Gezonde voeding en voedinsstoffen 2016. retrieved from http://www. voedingscentrum.nl/nl.aspx.

Wang, X., Wang, M., Ruan, J., and Zhan, H. (2016). The multi-objective optimization for perishable food distribution route considering temporal-spatial distance. Procedia Computer Science, 96:1211-1220.

Weber, C. L. and Matthews, H. S. (2008). Food-miles and the relative climate impacts of food choices in the United States.

Weidema, B. P., Bauer, C., Hischier, R., Mutel, C., Nemecek, T., Reinhard, J., and Wernet, G. (2013). Overview and methodology. Data quality guideline for the ecoinvent database version 3. Ecoinvent Report 1 (v3). St. Gallen: The ecoinvent Centre. Swiss Centre for Life Cycle Inventories. 1-159.

Weidema, B. P. and Eder, P. (2008). Environmental improvement potentials of meat and dairy products. JRC Scientific and Technical Reports.

Wernet, G., Bauer, C., Steubing, B., Reinhard, J., Moreno-Ruiz, E., and Weidema, B. (2016). The ecoinvent database version 3 (part i): Overview and methodology. The International Journal of Life Cycle Assessment, 21(9):1218-1230.

Wilson, N., Nghiem, N., Mhurchu, C. N., Eyles, H., Baker, M. G., and Blakely, T. (2013). Foods and dietary patterns that are healthy, low-cost, and environmentally 
sustainable: A case study of optimization modeling for New Zealand. PloS one, 8(3):e59648.

Wu, G., Fanzo, J., Miller, D. D., Pingali, P., Post, M., Steiner, J. L., and Thalacker-Mercer, A. E. (2014). Production and supply of high-quality food protein for human consumption: Sustainability, challenges, and innovations. Annals of the New York Academy of Sciences, 1321(1):1-19.

Yu, M. and Nagurney, A. (2013). Competitive food supply chain networks with application to fresh produce. European Journal of Operational Research, $224(2): 273-282$.

Yu, P.-L. (1973). A class of solutions for group decision problems. Management Science, 19(8):936-946.

Zarandi, M. H. F., Hemmati, A., Davari, S., and Turksen, I. B. (2013). Capacitated location-routing problem with time windows under uncertainty. Knowledge-Based Systems, 37:480-489.

Zeleny, M. (1973). Compromise programming. Multiple Criteria Decision Making, pages 262-301.

Zhao, Q.-H., Chen, S., and Zang, C.-X. (2008). Model and algorithm for inventory/routing decision in a three-echelon logistics system. European Journal of Operational Research, 191(3):623-635.

Zhou, L., Baldacci, R., Vigo, D., and Wang, X. (2018). A multi-depot two-echelon vehicle routing problem with delivery options arising in the last mile distribution. European Journal of Operational Research, 265(2):765-778.

Zhu, X. and van Ierland, E. C. (2004). Protein chains and environmental pressures: A comparison of pork and novel protein foods. Environmental Sciences, 1(3):254-276.

Zhu, Z., Chu, F., Dolgui, A., Chu, C., Zhou, W., and Piramuthu, S. (2018). Recent advances and opportunities in sustainable food supply chain: A model-oriented review. International Journal of Production Research, 56(17):5700-5722. 



\section{Summary}

The food system is a complex global structure, comprising an intrinsic web of interrelated supply chain and consumption activities. As such, it is deeply embedded in our society, contributing significantly to our economy and well-being. However, its current setup also leaves a considerable environmental footprint, by depleting valuable resources and polluting the planet, thus threatening the food security of future generations. A growing population and increasing standard of living further contribute to these environmental threats, while unhealthy consumption behaviour causes a rise in obesity and non-communicable diseases.

This thesis shows how Operations Research approaches can contribute to finding solutions for a more sustainable food system. By applying mathematical optimisation and solution techniques, the research reconsiders the system's setup and evaluates possible alternative scenarios in order to address the current challenges. In order to provide a holistic view of the system and consider the perspective of different decision makers, different decision levels are presented and investigated in this thesis.

In Chapter 2, the food system is considered from a network perspective, taking into account relations between consumption and supply chain decisions. In this context, a network flow problem is proposed to investigate the shifting towards a more plant-based dietary consumption on the basis of a number of alternative scenarios. The problem includes several echelons and interlinkages between different food supply chains by integrating sourcing, production and transportation decisions within a common framework. Consumption decisions are incorporated in the form of different types of consumer demands, maintaining a sufficient dietary intake level for the Dutch population. The problem is illustrated, with the help of real-life LCA data, on the basis of a case study and solved for different objectives using a linear programming approach. A multi-objective analysis, based on the $\epsilon$-method and compromise programming, provides further insights into the existing trade-offs between the investigated environmental and economic objectives. The findings show that a plant-based dietary consumption holds the largest potential to reduce the environmental impact of the food system, while indicating the implications of such a shift for the supply chain configuration. Moreover, insights are provided on the allocation 
and shifting of burdens in the system depending on the chosen impact indicator.

Chapter 3 continues the investigation at the network level from a more nutritional perspective. Building on the modelling approach of Chapter 2, the research is more restrictive in terms of dietary intake choices and applies tighter nutritional bounds. Minimising several environmental impact indicators, the resulting consumption alternatives are compared with regards to environmental footprint, product mix and the underlying supply chain configuration. Given the nutritional emphasis, the comparison also includes the effect of different alternatives on the overall dietary intake. The findings indicate benefits of shifting towards a more plant-based consumption both from a health perspective as well as from an environmental standpoint. Highlighting the connection between meat and dairy products, the research also shows the importance of taking product relations into account.

Chapter 4 shifts the focus to operational aspects in the system, by addressing inventory management and routing decisions in the context of innovative last mile distribution concepts for perishable products. Assuming a two-echelon framework, the considered inventory-routing problem consists of a supplier, an intermediary depot and individual customer locations. The supplier delivers products to the depot, where storage may occur and from which they are then delivered by smaller vehicles to the customer locations. Storage at the depot incurs a holding costs, while customer preferences and availability for delivery are specified in the form of customer delivery patterns. Minimising total transportation and holding cost, the problem is formulated as a mixed-integer program. Given the complexity of the problem, a two-stage matheuristic is proposed for finding solutions on the basis of an adaptive large neighbourhood search and a reduced version of the problem. Three variants of the heuristic are compared in terms of their computational performance on a variety of randomly generated instances. Focusing on computational aspects, the findings highlight the importance of taking the cost structure into account when choosing the most suitable solution approach.

Another last mile delivery concept for the distribution of fresh products is considered in Chapter 5, investigating the effect of alternative delivery locations, in the form of customer pick-up points, on daily routing operations. Due to the existence of customer pick-up points, customers can either be delivered directly at the customer location, or indirectly through a pick-up point, where products are stored until pick-up occurs. Customer pick-up points allow for more flexibility, as direct delivery is restricted by tight time windows. However, storage is capacitated and requires cooling, resulting in an additional cost to operate the facility. Minimising total transport and storage cost, the locationrouting problem is formulated using a mixed-integer program and solved by means of an adaptive large neighbourhood search. The heuristic is tested on a set of benchmark instances. The results from these experiments indicate the potential of incorporating cus- 
tomer pick-up stations in last mile distribution systems for fresh products to save costs and make delivery operations more efficient.

Zooming further into consumer plates, Chapter 6 looks at individual product concepts and how to design more sustainable alternatives to currently consumed products. Revisiting the shifting towards a more plant-based dietary consumption, the study focuses on the design of meat replacers with an equivalent nutritional contribution as chicken or beef, with regards to a set of key nutrients. Particular attention is given to protein quality and iron absorbability. Minimising different environmental impact indicators, a number of alternatives are proposed, as solutions to the linear programming based blending problem. Environmental impacts of ingredients are quantified through life-cycle assessment (LCA) data. The findings show that the largest impact reduction can be achieved through a vegan replacement, except for water use where the best result is provided by an insect-based replacement. The results further indicate the potential benefits of soy as an ingredient, due to its favourable amino acid composition.

Chapter 7 presents a general discussion and conclusion following from the main findings of this thesis.

The thesis highlights the multifaceted nature of challenges in the current food system and demonstrates the ability of Operations Research approaches to contribute to decision making on different levels in the system. At the same time, synergies between Operations Research and other food related disciplines give rise to new optimisation problems with practical implications, providing insights into different application areas. 



\section{Publications}

\section{In this dissertation}

Van Mierlo, K., Rohmer, S., \& Gerdessen, J. C. (2017). A model for composing meat replacers: Reducing the environmental impact of our food consumption pattern while retaining its nutritional value. Journal of Cleaner Production, 165, 930-950.

Rohmer, S. U. K., Gerdessen, J. C., Claassen, G. D. H., Bloemhof, J. M., \& van't Veer, P. (2018). A nutritional comparison and production perspective: Reducing the environmental footprint of the future. Journal of Cleaner Production, 196, 1407-1417.

Rohmer, S. U. K., Gerdessen, J. C., \& Claassen, G. D. H. (2019). Sustainable supply chain design in the food system with dietary considerations: A multi-objective analysis. European Journal of Operational Research, 273(3), 1149-1164.

Rohmer, S. U. K., Claassen, G. D. H., \& G. Laporte (2019). A two-echelon inventory-routing problem for perishable products. Computers \& Operations Research, $107,156-172$.

\section{Other scientific publications}

Rohmer, S., \& Billaut, J. C. (2015). Production and outbound distribution scheduling: a two-agent approach. In Proceedings of the International Conference on Industrial Engineering and Systems Management (IESM'2015). Sevilla, Spain. 



\section{A final toast - Raising the glasses}

This thesis presents the final product of my PhD and as such covers the main results of my research, gives a taste of my scientific work and maybe even provides you with some food for thought. However, looking at the final product, we often fail to capture the underlying system that made this whole thing possible and neglect the impact of the process that led to its creation. These pages are therefore dedicated to my support system and all the people who have helped to realise this final product.

First and foremost, I would like to express my sincere gratitude to my daily supervisors, Frits and Joke, for their never-ending support and encouragement that has led me to pursue my ambitions and expand my horizon during these four years of research. The two of you always had my back, an open ear and my happiness in mind, no matter the challenges I was facing. Your comprehension and understanding towards my many side projects has furthermore contributed to make these years a multifaceted, exciting and unforgettable experience.

Part of my supervision team, I also want to thank my two promotors, Jacqueline and Pieter, for their valuable input and our shared discussions throughout the years. Despite your busy schedules, you challenged me, kept me on my toes and let me see things from different perspectives.

Moreover, I want to thank my co-authors, Klara and Gilbert, who contributed to this final product and from whom I learned a lot in the process. Klara, you were a remarkable student and your dedication and hard work resulted in our shared publication. Supervising you was a pleasure that provided me with a fresh perspective on the scientific process and made me become a more complete academic. Gilbert, your invitation to Montreal has resulted in one of the most rewarding experiences of my $\mathrm{PhD}$, during which you have been an exceptional mentor with an abundance of experience and ideas. Your efficiency and overarching view of the field are only some of the things I hope to emulate on my future career path.

In relation to the work presented in this thesis, I also want to thank Blonk consultants for their involvement in the project and the provision of data that helped to underpin my work. Special mention deserves Roline Broekema in this context for taking the time to share her experience and knowledge with me.

Aside from the people directly involved in the creation of this thesis, I also want to mention the people who were a part of my production environment, contributed to smooth operations and made the hours in the office so much more enjoyable. The first thanks in this context goes to the secretaries and administrators for aligning agendas, battling with administrative systems, booking flights and so many other things that often go unnoticed. Thank you for the underlying support that made things so much easier. 
Of course this production environment also includes all my other colleagues who have kept me company and been a source of inspiration along this journey. Many of you have become friends along the way and I appreciate all the breaks that have helped to clear my mind and restored my focus. Thanks for letting me pick your brains and distract you from your work!

But life is not just work and every production system needs some down time and proper maintenance in order to function smoothly. For this, I want to thank my friends for checking in on me, believing in me and restoring my spirits. You are my family away from home and responsible for some of the best memories along this way, I couldn't have done it without you! Thank you for all the good times, the laughs, the food, the cookies and of course for dealing with my frustrations.

Last but definitely not least, I want to thank my family for laying the foundation and planting the seeds from which I could grow and develop into the person I am today. You have always encouraged me to be independent, make my own choices and be whoever I want to be. I am grateful for your unconditional love and support that has shaped my character and inspired me to follow my dreams and explore the world. Thank you for being my safe haven to return home to and providing me with your guidance along the way. 
Sonja Ursula Katharina Rohmer

Wageningen School of Social Sciences (WASS)

Completed Training and Supervision Plan

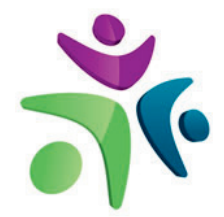

Wageningen School

of Social Sciences

\begin{tabular}{|c|c|c|c|}
\hline Name of the learning activity & Department/Institute & Year & ECTS \\
\hline \multicolumn{4}{|l|}{ A) Project related competences } \\
\hline Robust Optimisation & LNMB & 2016 & 4 \\
\hline Stochastic Programming & LNMB & 2016 & 1 \\
\hline $\begin{array}{l}\text { Advanced Topics of Management } \\
\text { Engineering I }\end{array}$ & PPGEP - UFPE & 2016 & 2 \\
\hline Transport Logistic Modelling & GP OML & 2016 & 4 \\
\hline $\begin{array}{l}\text { Quantitative Modelling and Analysis of } \\
\text { Supply Chains }\end{array}$ & GP OML & 2019 & 1 \\
\hline \multicolumn{4}{|c|}{ B) General research related competences } \\
\hline Introduction course & WASS & 2015 & 1 \\
\hline Reviewer activities for scientific journal & Elsevier & 2018 & 1 \\
\hline The Future for Food Systems & IFPRI workshop & 2016 & 1 \\
\hline Healthy, Sustainable and Affordable Diets & MCDM & 2017 & 1 \\
\hline Towards healthy \& sustainable food & WUR & 2018 & 1 \\
\hline
\end{tabular}

systems... in an urbanising world

C) Career related competences/personal development

Teaching assistance for Operations

ORL

$2015-2018 \quad 2$

Research and Logistics courses

Supervision of BSc and MSc thesis

ORL

$2015-2019 \quad 2$

students

$12^{\text {th }}$ MCDM Summer School

Recife, Brazil

2016

2.1

'Sustainable supply chain design in the food

IFORS, Quebec, Canada

2017

1

system with dietary considerations: A multi-

objective approach'

'Multi-Objective Optimisation for the Design

of Sustainable Food Systems in the context

of Dietary Guidelines “

'Design of Green Food Systems under

Nutritional Considerations '

'A two-echelon inventory-routing problem

for the last mile delivery of perishable

MCDM, Ottowa, Canada

2017

products'

' A Location-Routing Problem with Delivery

Options and Time-Windows for the Last Mile

JOPT, Montreal, Canada

2017

1

Delivery of Fresh Products'

Internship at CIRRELT/ HEC Montreal

EURO, Valencia, Spain

2018

1

VeRoLog, Seville, Spain

2019

1

Total

CIRRELT

2017

6

${ }^{*}$ One credit according to ECTS is on average equivalent to 28 hours of study load 
This thesis is part of the $\mathrm{PhD}$ thesis series of the Beta Research School for Operations Management and Logistics (onderzoeksschool-beta.nl) in which the following universities cooperate: Eindhoven University of Technology, Maastricht University, University of Twente, VU Amsterdam, Wageningen University and Research, KU Leuven and Universiteit Hasselt.

The work presented in this dissertation was conducted within the framework of the Greendish project (ALWGroen.2014.017), funded by the Netherlands Organisation of Scientific Research (NWO). 

